\title{
Copepods from Warm-Core Ring 82-H
}

\author{
by \\ Nancy J. Copley, Peter H. Wiebe, and Timothy J. Cowles \\ Woods Hole Oceanographic Institution \\ Woods Hole, Massachusetts 02543
}

July 1989

\section{Technical Report}

Funding was provided by the National Science Foundation through grant Number OCE 80-12748, OCE 85-08350, OCE 87-09962, OCE 80-19055, and OCE 80-17271.

Reproduction in whole or in part is permitted for any purpose of the United States Government. This report should be cited as:

Woods Hole Oceanog. Inst. Tech. Rept., WHOI-89-24.

Approved for publication; distribution unlimited.

\section{Approved for Distribution:}

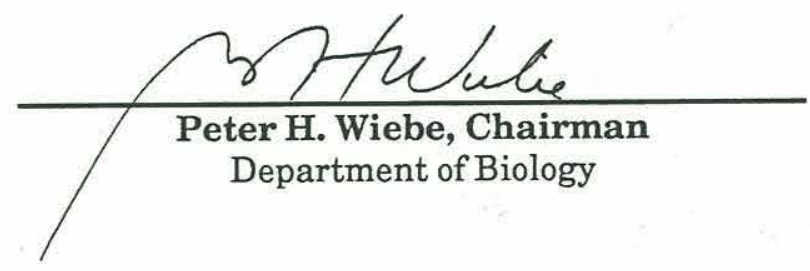





\begin{abstract}
Net tows were collected with a Multiple Opening/Closing Net Environmental Sampling System (MOCNESS) carrying twenty $1-\mathrm{m}^{2}$ nets in October 1982 in and near warm-core ring 82-H in the North Atlantic (RV/Knorr cruise 98). This report includes the species list and abundance tables of the copepods found in five of the tows. There are four types of abundance tables: raw data, standardized to \#/1000 $\mathrm{m}^{3}$, integrated $\# / \mathrm{m}^{2}$ to 1000 $\mathrm{m}$ depth, and cumulative percents over the depth of the tows.
\end{abstract}




\section{Table of Contents}

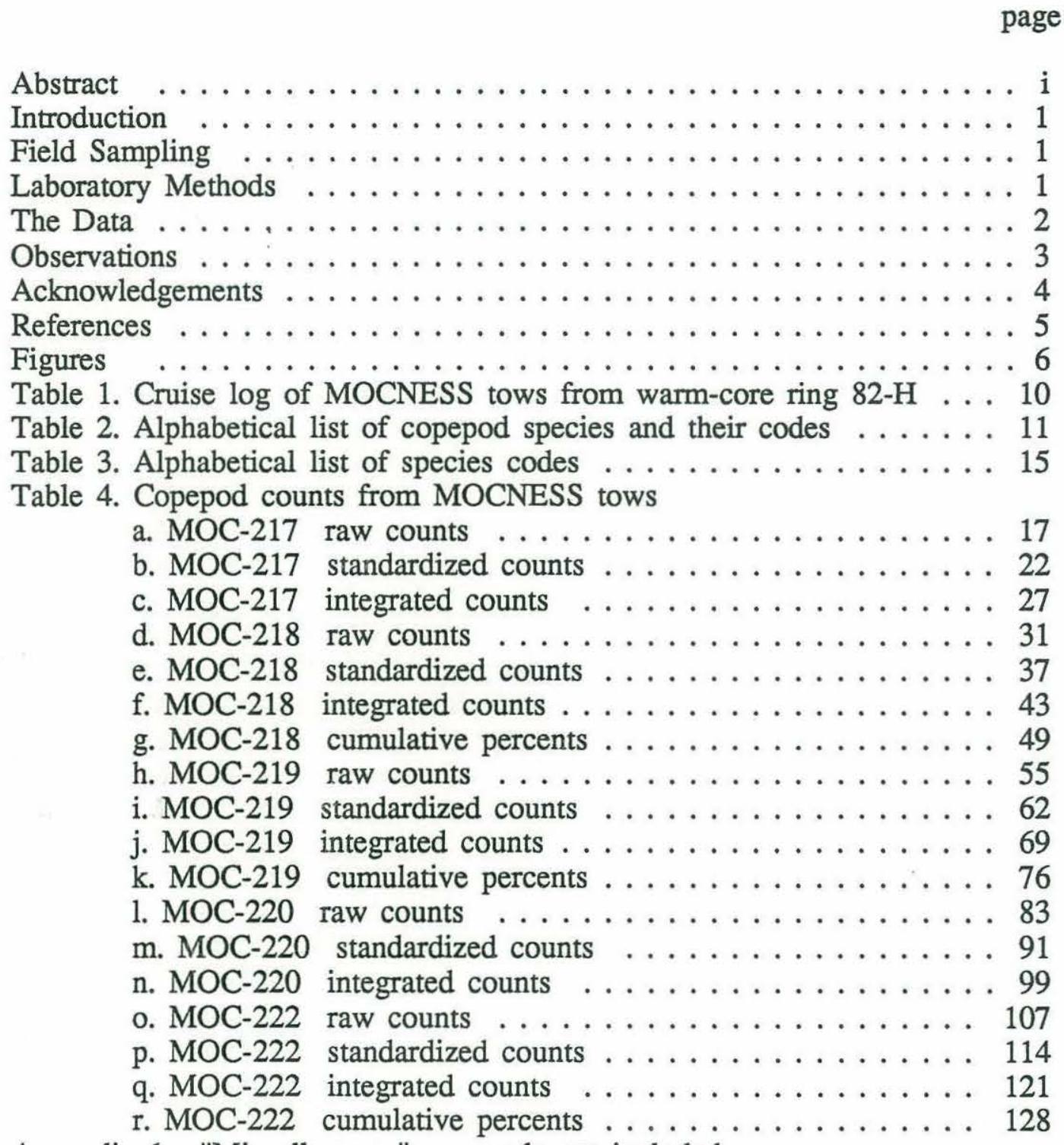

Appendix 1. "Miscellaneous" copepods not included

in table 4: some counts and notes 


\section{Introduction}

The objective of this work was to determine the abundance and distribution of copepods associated with Gulf Stream warm-core ring $82-\mathrm{H}$ in the North Atlantic. Copepods were identified from five net tows collected with a double $1 \mathrm{~m}^{2}$ Multiple Opening/Closing Net Environmental Sampling System (MOCNESS).

This report includes the species list and abundance tables of the copepods found in five of the tows. There are four types of abundance tables: raw data, standardized to \#/1000 $\mathrm{m}^{3}$, integrated $\# / \mathrm{m}^{2}$ to $1000 \mathrm{~m}$ depth, and cumulative percents over the depth of the tows. An appendix lists some of the copepods found in the samples which were not included in the main set of tables.

\section{Field Sampling}

Warm-core ring $82-\mathrm{H}$ was sampled before, during and after its formation in September and October 1982 on RV/Knorr cruise 98. Samples were collected with a double Multiple Opening/Closing Net Environmental Sampling System (MOCNESS) carrying 20 nets of 335 $\mu \mathrm{m}$ Nitex nylon mesh; net opening was $1 \mathrm{~m}^{2}$ (Wiebe et al, 1985b). Barber and Wiebe (1985) contains further details on the MOCNESS system, sampling strategy, tow information for all the warm-core ring tows (dates, times, positions, depth of $10^{\circ}$ isotherm, volume filtered/net), and physical data (temperature and salinity). Wiebe et al (1985a) also gives details of the sampling area and methods. The dates and locations of MOC 217, 218, 219, 220 , and 222 are listed in Table 1 . Figure 1 shows the geographic position of ring $82-\mathrm{H}$ shortly after formation and the positions of the tows relative to the ring center which moved substantially to the west after formation.

All tows but MOC 217 were taken from the ring center area. MOC 217 was a towyo (series of oblique hauls from surface to depth) cycled between $0 \mathrm{~m}$ and approximately $125 \mathrm{~m}$ beginning in waters of ring origin, passing through a front on the outer edge of the ring and into the surrounding Slope Water. Nets were alternately closed at the bottom of the tow and then three nets were used to sample the upward haul (Figure 2). These nets sampled below a subsurface temperature maximum, to the bottom of the mixed layer, and the surface mixed layer. MOC 218 and 219 sampled from $0 \mathrm{~m}$ to $1000 \mathrm{~m}$ with MOC 218 taken at night and MOC 219 during the day. Nets were closed every $100 \mathrm{~m}$ between $1000 \mathrm{~m}$ and $200 \mathrm{~m}$ and every $25 \mathrm{~m}$ from $200 \mathrm{~m}$ to the surface. MOC 220 was a towyo from $0 \mathrm{~m}$ to $100 \mathrm{~m}$ beginning in the daylight and ending after dark and MOC 222 was a nighttime high resolution oblique tow to $160 \mathrm{~m}$ (nets closed every $10 \mathrm{~m}$ ). Wiebe, Copley, and Boyd (in press) provide a more detailed explanation of the field setting and sampling technique.

\section{Laboratory Methods}

The contents of each net were preserved in $10 \%$ formalin buffered with sodium tetraborate and sorted using a Wild M-5 dissecting microscope. For further species identification a compound microscope was sometimes used. Large samples were split to no more than $1 / 16$ aliquot in a Folsom plankton splitter. The samples were sorted over a $1-1 / 2$ 
year period (April 1985 - December 1986) during which the emphasis changed from enumerating only the more common species to trying to identify all the copepods. A total of over 130 species were found. All species were identified in MOC 219 and 222; about 94 species were found in MOC 219 and 69 in MOC 222. Almost all copepods were identified in MOC 220; 56 species were recorded. MOC 217 lists 45 species and MOC 218 has 87; all species were not identified in these two tows so the absence of any species from a particular tow from Table 2 does not necessarily mean it was not present. However, a total count of copepods was made on all samples. See Table 2 for a complete listing of species (alphabetized by genus) identified from the five tows.

\section{The Data}

Tables of raw, standardized, integrated, and cumulative percent data for the most abundant species and some of the less common but large species are included in this report (Tables 4a-r). Species are listed in taxonomic order. Females, males and copepodites were identified separately for each species except Lucicutia spp. and Acartia spp. where they are combined. Copepod species categories (female, male, copepodite) are given a three letter code on the tables. The code is preceded by an ' $\mathrm{f}$ ' for female, ' $\mathrm{m}$ ' for male, and ' $c$ ' for copepodite. If none of these precedes the code, females, males, and copepodites were grouped (luci for Lucicutia spp., oith for Oithona spp.). The raw data tables show just the species code; in the standardized tables, the code is preceeded by a '\#', integrated tables are identified by an ' $\mathrm{i}$ ', and cumulative percent tables have a ' $\%$ ' before each species code. Data in the 'raw' tables are counts for the aliquot. Aliquot size is indicated; an '8' in the 'aliq' column means that a $1 / 8$ th split was sorted and the values on the table are the number of individuals found in that eighth split. Table 3 lists codes alphabetically with their species names. Appendix 1 lists some of the copepods that are included in the abundance table 4 under the heading 'misc'. These are the more rare adults, and copepodites of a group of similar species; c Spinocalanus spp. for example.

From the original counts, standardized values were calculated for the number of individuals $/ 1000 \mathrm{~m}^{3}$ of each species category in each net. The integrated number of individuals $/ \mathrm{m}^{2}$ to a depth of $1000 \mathrm{~m}$ and the cumulative percent of each category was calculated as well. The formulae are as follows:

$$
\text { Standard: } \quad \text { STD }=(\text { RAW/ALIQ }) *(1000 / \text { VOLFIL })
$$

where RAW is the \# copepods counted in the split sample, ALIQ is the reciprocal of the aliquot ( 8 for an eighth split: ALIQ is given in the data tables in this form), and VOLFIL is the volume of water filtered in a strata.

$$
\begin{aligned}
& \text { Integrated: } \quad \mathrm{I}_{\mathrm{B}}=\sum_{\mathrm{i}=1}^{\mathrm{n}} \mathrm{b}_{\mathrm{i}} * \mathrm{~h}_{\mathrm{i}} \\
& \text { Cumulative \%: } \mathrm{C}_{\mathrm{i}}=\frac{\mathrm{b}_{\mathrm{i}} * \mathrm{~h}_{\mathrm{i}}}{\mathrm{I}_{\mathrm{B}}} * 100+\mathrm{C}_{(\mathrm{i}-\mathrm{-})}
\end{aligned}
$$


where $I_{B}=$ integrated \#/m² to $1000 \mathrm{~m}, b_{i}=\#$ in strata $i\left(\# / 1000 \mathrm{~m}^{3}\right), h_{i}=$ height of strata in meters, and $n=$ number of strata sampled with the count starting at the surface. The vertical distribution of a copepod species category is expressed in terms of the cumulative percent of that category in the $\mathrm{i}^{\text {th }}$ strata, $\mathrm{c}_{\mathrm{i}}$, beginning with the surface strata (from Barber and Wiebe, 1985). Integrated counts for MOC 217 were found by combining the three nets of the uphaul; down-hauls sampled the entire range so were computed alone. The data is found in Table $4 \mathrm{c}$ with haul number listed instead of net number as the other four MOCNESS tows show.

A 1986 unpublished technical report by Flierl ("The Warm Core Rings Database Routines", available in the WHOI reference library) describes the data format. All data in the database are treated as tables of numbers. The header for each column is stored with the table and serves to identify visually the data in that column. The data must be numeric; the value 999 is used to identify a missing data item. The data are stored in MS-DOS ASCII files containing headers and data in a single column using the Flierl database format: the first line has the number of columns (NC), the next NC lines have the table headings, and the following groups of NC lines contain successive rows of the table. A disk with all data presented in this report is on file in the WHOI reference library along with copies of this report. Requests for permission to use the data should be obtained from the authors of this report.

\section{Observations}

The MOCNESS went through a frontal region on tow 217. The species composition changed as the nets went from the warm ring to the cold Slope water. Three species were dominant in the warmer, ring center water: Undinula vulgaris, Euchaeta marina, and Candacia pachydactyla. Several calanoid species were dominant in the colder, slope water: Nannocalanus minor, Calanus finmarchicus, C. tenuicornis (stage V), Rhincalanus cornutus, Clausocalanus arcuicornis, Lucicutia spp., Centropages typicus, C. violaceus, Haloptilus longicornis, Candacia armata, and also Oithona spp. A few species were more abundant on either side of the front: Scolecithrix danae, Centropages furcatus, and some cyclopoids. Results of the towyo taken across a front on the outer edge of the ring (MOC 217) and the towyo made in ring center at sunset (MOC 220) are discussed in detail in Wiebe et al (in press).

In MOC 219, the species composition from 300-1000 meters was noticeably more diverse. Larger species (Calanus finmarchicus, C. hyperboreus, Eucalanus elongatus, Rhincalanus cornutus, Undeuchaeta spp.) were more abundant than in the upper 300 meters but overall abundance was lower.

In MOC 222, the fine scale tow which sampled every $10 \mathrm{~m}$ from $160 \mathrm{~m}$ to the surface, the vertical distribution of the five Candacia species was distinctive. C. pachydactyla males and females were found from the surface to $70 \mathrm{~m}$. One male $C$. ethiopica was found between 10 and $20 \mathrm{~m}$. C. longimana females occurred from $20 \mathrm{~m}$ to $100 \mathrm{~m}$ and the males from 30 $\mathrm{m}$ to $90 \mathrm{~m}$. C. curta females were caught between $70 \mathrm{~m}$ and $120 \mathrm{~m}$ and C. ketchumi females were found between $100 \mathrm{~m}$ and $160 \mathrm{~m}$; the males were found from $120 \mathrm{~m}$ to $160 \mathrm{~m}$. In general, co-specific male and female Candacia shared similar strata while different species 
overlapped very little. Other congeners exhibited similar segregation in MOC 222: Scolecithrix danae $(0-50 \mathrm{~m})$ and $S$. bradyi $(30-150 \mathrm{~m})$ seemed to prefer different regions and Acartia danae $(0-90 \mathrm{~m})$ and $A$. negligens $(80-160 \mathrm{~m})$ had little overlap. The Pleuromamma species did not show this tendancy at all, with all five species co-occuring (Figure 3 ).

The distribution of some species of copepods in the high resolution tow (MOC 222) corresponded well with the temperature and salinity curves. From the surface to about $60 \mathrm{~m}$, the temperature remained about $24.8^{\circ} \mathrm{C}$. The seasonal thermocline began at about $65 \mathrm{~m}$ and temperature gradually dropped to $18^{\circ} \mathrm{C}$ at $160 \mathrm{~m}$ (Figure 4). Some species were concentrated above 60 to $80 \mathrm{~m}$, others mostly occured deeper, while others were found more or less evenly over the entire $160 \mathrm{~m}$.

\begin{tabular}{lll}
\multicolumn{1}{c}{ mixed layer } & below thermocline & \multicolumn{1}{c}{ both } \\
Acartia danae & Acartia negligens & Clausocalanus arcuicornis \\
Acrocalanus longicornis & Aetideus armatus & C. furcatus \\
Candacia pachydactyla & Candacia ketchumi & Heterorhabdus papilliger \\
Centropages violaceus & Chirundina streetsi & Neocalanus robustior \\
Eucalanus attenuatus & Euaetideus giesbrechti & Pleuromamma abdominalis \\
Euchirella messinensis & Gaetanus minor & P. gracilis \\
Nannocalanus minor & Haloptilus longicornis & Scaphocalanus nr. amplius \\
Pleuromamma piseki & Heterorhabdus spinifrons & S. nr. longicornis \\
Pontellina plumata & Lucicutia clausi & Undeuchaeta plumosa \\
Scolecithrix danae & Phyllopus helgae & \\
Temora stylifera & Scolecithricella vittata & \\
Undinula vulgaris & &
\end{tabular}

Some species were concentrated in and just below the thermocline $(60-90 \mathrm{~m})$ : Calanus tenuicornis, Lophothrix latipes, Mecynocera clausi, Phaenna spinifera, Pontellina plumata and Scottocalanus securifrons.

\section{Acknowledgements}

We would like to thank Al Morton who provided valuable help in making the tows and maintaining the MOCNESS equipment. Valerie Barber and Steven Boyd assisted in sample collection. Mar Tamse provided analytical assistance. 


\section{References}

Barber, V. and P. H. Wiebe. 1985. Zooplankton biomass and related MOCNESS data for tows taken on the 1981-82 warm-core rings cruises. WHOI Technical Report 85-3 (164 pp.)

Flierl, G. R. The Warm Core Ring Database Routines. unpublished technical report. June 1986.

Wiebe, P. H., G. R. Flierl, C. Davis, V. Barber, and S. H. Boyd. 1985a. Macrozooplankton biomass in Gulf Stream warm-core rings: Spatial distribution and temporal changes. J. Geophys. Res. 90: 8885-8901.

Wiebe, P. H., A. W. Morton, A. M. Bradley, R. H. Backus, J. E. Craddock, V. Barber, T. J. Cowles, G. R. Flierl. 1985b. New developements in the MOCNESS, an apparatus for sampling zooplankton and micronekton. Marine Biology 87: 313-323.

Wiebe, P. H., N. J. Copley, and S. H. Boyd. Coarse-scale horizontal patchiness and vertical migration of zooplankton in newly formed Gulf Stream warm-core ring 82-H. (submitted to Deep-Sea Research) 
Figure 1. (a) Postion of Gulf Stream meander $82-\mathrm{H}$ just prior to ring formation (solid line) and ring 82-H shortly after formation (closed circle). (b) Positions of the MOCNESS tows taken on RV/KNORR cruise 98 to meander/ring $82-\mathrm{H}$ relative to ring center. Corrected from Wiebe et al (1985); (from Wiebe, Copley, Boyd, in press).
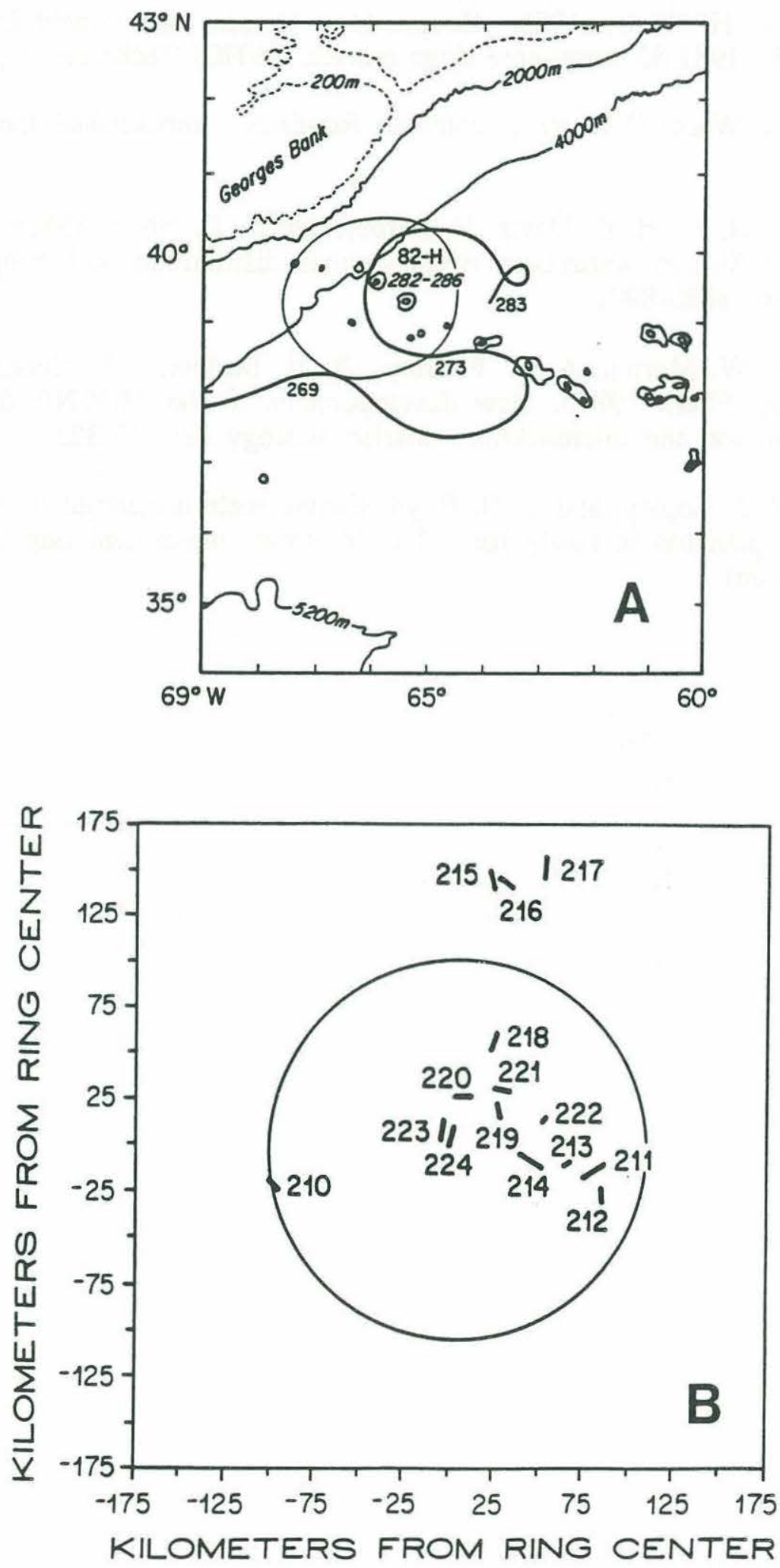
Figure 2. Horizontal section of (a) temperature and (b) salinity taken on towyo MOC 217 in the fringe region of ring $82-\mathrm{H}$. (c) towyo net trajectory - cross lines on the up-track mark the closing and opening of nets. Horizontal section of (d) temperature and (e) salinity on towyo MOC 220 at the center of the ring. (f) towyo net trajectory of MOC 220 (from Wiebe, Copley, Boyd, in press).
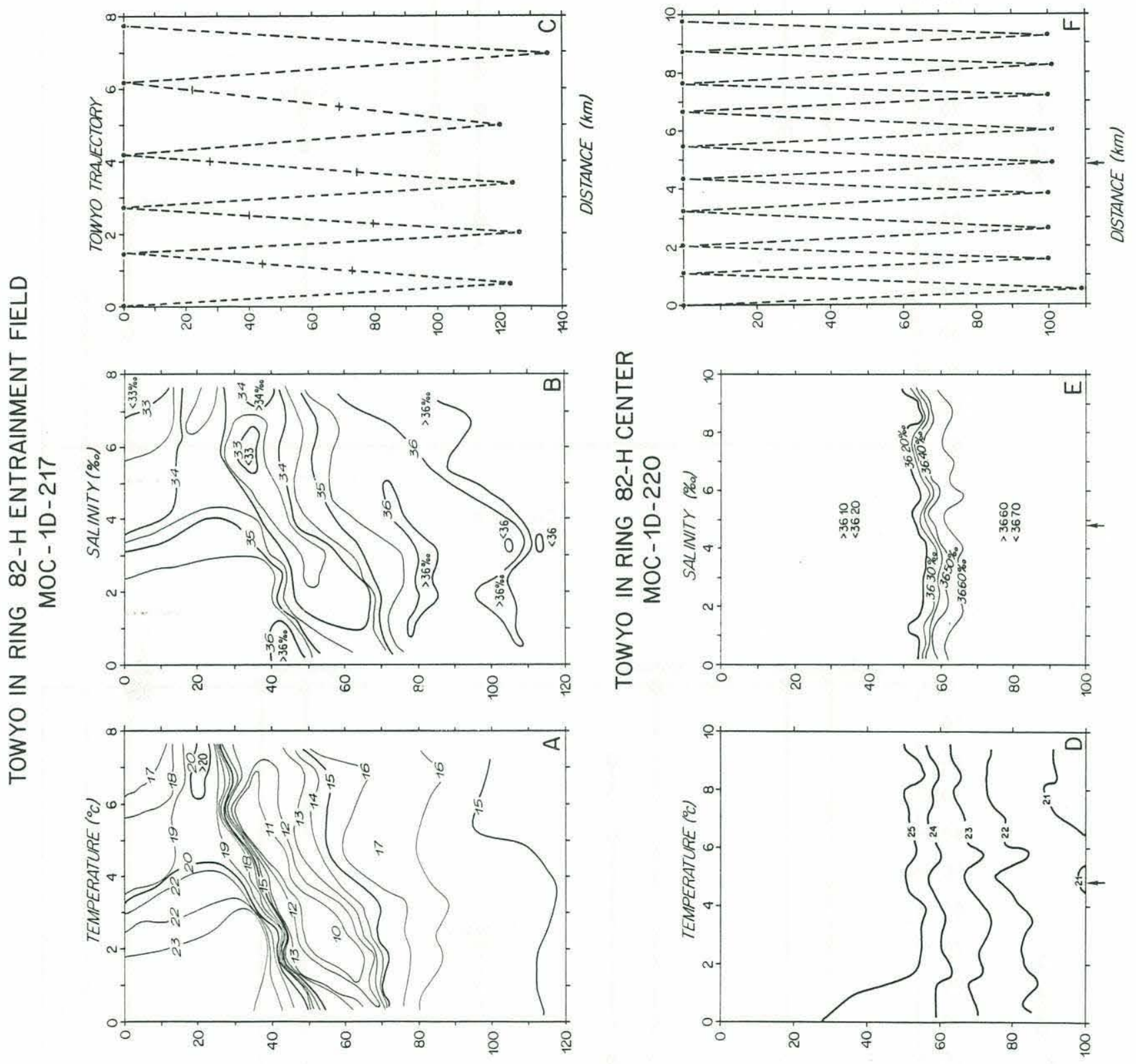

(w) $H \perp d \exists O$ 
Figure 3. Vertical distributions from surface to $160 \mathrm{~m}$ of (a) Candacia spp. and (b) Pleuromamma spp. from high resolution night tow MOC 222 in ring center.
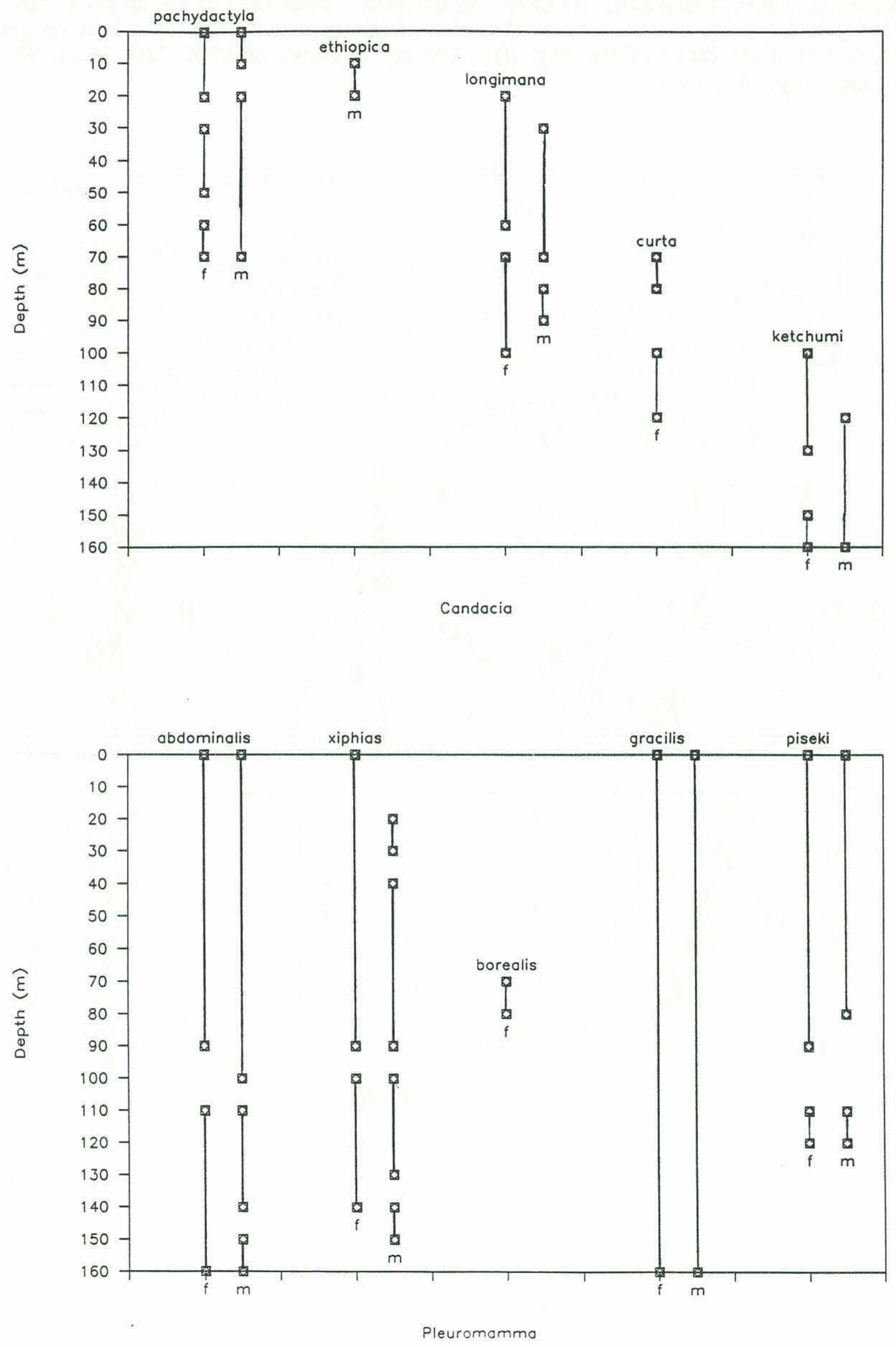
Figure 4. Temperature and salinity profiles from MOC 222.

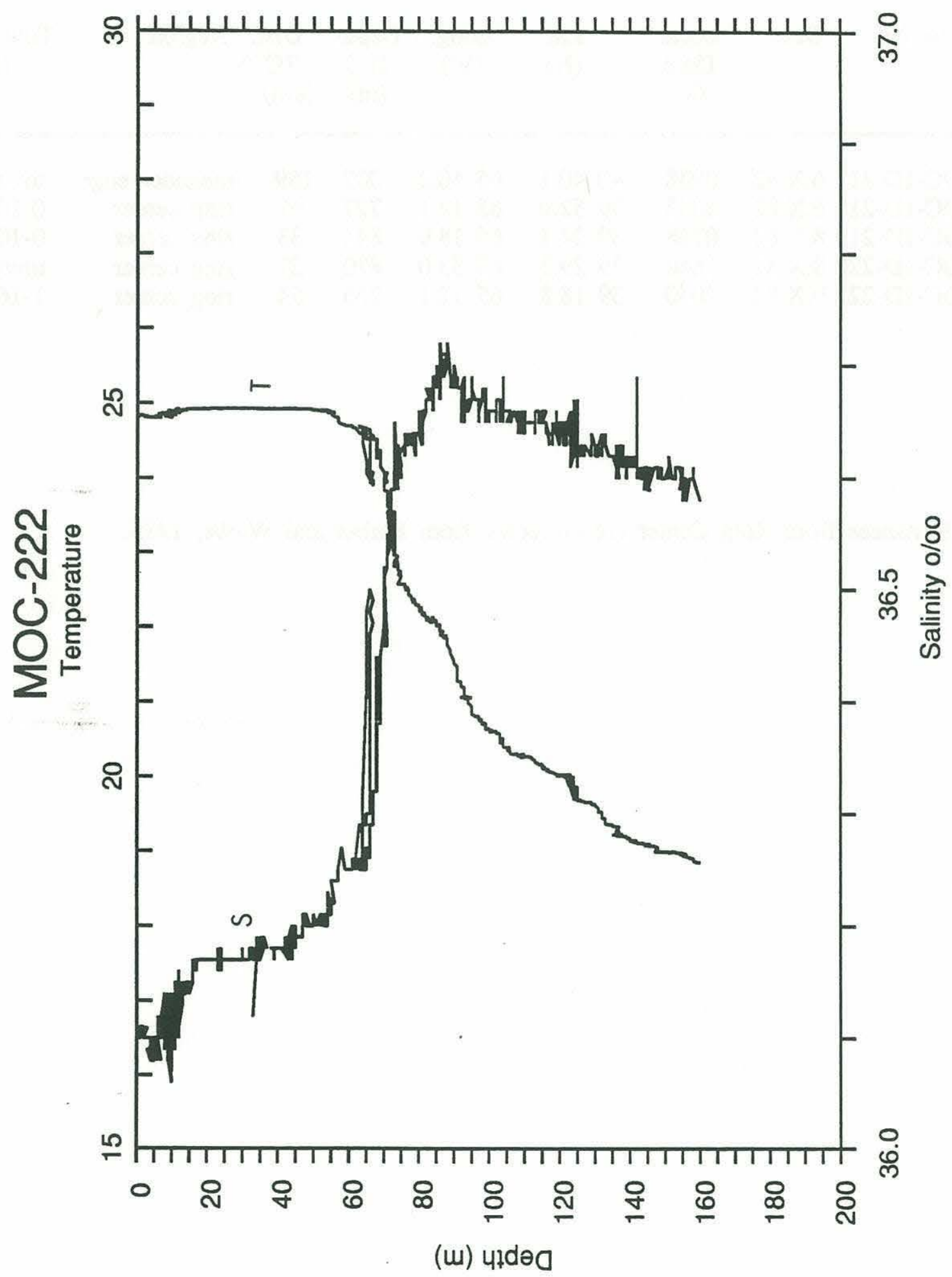


Table 1. Cruise log of MOCNESS tows from warm-core ring 82-H. Latitude and longitude are for the starting positions of the tow.

Tow No. Date Local Lat. Long. Depth Dist. Region

$$
\text { Time (N) (W) 10C RC * }
$$

(h) (m) (km)

Tow Depth

(m)

\begin{tabular}{|c|c|c|c|c|c|c|c|}
\hline MOC-1D-217 6.X.82 & 0938 & 4040.1 & 6550.2 & 307 & 159 & meander edge & towyo \\
\hline MOC-1D-218 6.X.82 & 2043 & 3952.9 & 6512.1 & 727 & 61 & ring center & $0-1000$ \\
\hline MOC-1D-219 8.X.82 & 0728 & 3924.1 & 6518.9 & 844 & 33 & ring center & $0-1000$ \\
\hline MOC-1D-220 8.X.82 & 1640 & 3929.3 & 6533.0 & 820 & 27 & ring center & towyo $0-100$ \\
\hline MOC-1D-222 9.X.82 & 2040 & 3918.8 & 6512.1 & 755 & 54 & ring center & $1-160$ \\
\hline
\end{tabular}

* Distances from Ring Center are corrected from Barber and Wiebe, 1985. 
Table 2. List of copepod species found in MOCNESS tows from warm-core ring 82-H and their codes. $\mathrm{x}=$ present, $-=$ not present, ? = may be present, not identified. Categories marked with an asterisk were not included in total species identified.

$\begin{array}{lllllll}\text { species name } & \text { code } & 217 & 218 & 219 & 220 & 222\end{array}$

Acartia danae

A. negligens

* Acartia spp.

Acrocalanus andersoni

A. longicornis

Aetideopsis multiserrata

Aetideus armatus

Amallothrix emarginata

A. laminata

A. nr. valida

Arietellus plumifer

A. setosus

* Augaptilidae spp.

Calanus finmarchicus

* stage V copepodites

* stage IV copepodites

* Cal., Neocal.,Undinula copepodites $=<$ III

C. hyperboreus

C. tenuicornis

Calocalanus parvoninus

Candacia armata

C. bipinnata

C. curta

C. ethiopica

C. ketchumi

C. longimana

C. pachydactyla

C. paenelongimana

* Candacia spp. copepodites

Centraugaptilus horridus

Centropages furcatus

C. typicus

C. violaceus

* Centropages spp. copepodites

Chirundina streetsi

Clausocalanus arcuicornis

C. furcatus

* Clausocalanus spp. males

nr. Ctenocalanus

\begin{tabular}{|c|c|c|c|c|c|}
\hline ada & $\mathrm{x}$ & $x$ & $\mathrm{x}$ & - & $x$ \\
\hline neg & $\mathrm{x}$ & $\mathrm{x}$ & $\mathrm{x}$ & $\mathrm{x}$ & $\mathrm{x}$ \\
\hline acar & $x$ & $x$ & - & - & - \\
\hline aca & $?$ & $\mathrm{x}$ & - & $\mathrm{x}$ & $\mathrm{x}$ \\
\hline \multirow[t]{2}{*}{ acr } & $\mathrm{x}$ & $?$ & $\mathrm{x}$ & $\mathrm{x}$ & $?$ \\
\hline & $?$ & $?$ & $x$ & $?$ & - \\
\hline \multirow[t]{2}{*}{ aar } & $x$ & $x$ & $x$ & $x$ & $\mathrm{x}$ \\
\hline & $?$ & $?$ & $\mathrm{x}$ & $?$ & - \\
\hline \multirow[t]{4}{*}{ lam } & - & $x$ & $x$ & $x$ & $\mathrm{x}$ \\
\hline & $?$ & $?$ & $x$ & $?$ & - \\
\hline & $?$ & $?$ & - & $x$ & $\mathrm{x}$ \\
\hline & $?$ & $?$ & $\mathrm{x}$ & $?$ & - \\
\hline augs & $\mathrm{x}$ & - & $\mathrm{x}$ & $x$ & $\mathrm{x}$ \\
\hline fin & $\mathrm{x}$ & $\mathrm{x}$ & $x$ & $\mathrm{x}$ & - \\
\hline vfin & $\mathrm{x}$ & $x$ & $\mathrm{x}$ & $\mathrm{x}$ & - \\
\hline cfin & $x$ & $\mathrm{x}$ & $\mathrm{x}$ & $\mathrm{x}$ & - \\
\hline ccal & $x$ & $\mathrm{X}$ & $x$ & $\mathrm{x}$ & $\mathrm{x}$ \\
\hline hyp & $x$ & $\mathrm{x}$ & $\mathrm{x}$ & - & - \\
\hline ten & $x$ & $\mathrm{X}$ & $x$ & $\mathrm{x}$ & $\mathrm{x}$ \\
\hline pav & $x$ & $x$ & $\mathrm{x}$ & $\mathrm{x}$ & $\mathrm{x}$ \\
\hline \multirow{2}{*}{ arm } & $x$ & $x$ & - & - & - \\
\hline & $x$ & - & - & - & $\mathrm{x}$ \\
\hline cur & - & - & - & $x$ & $\mathrm{x}$ \\
\hline eth & - & $x$ & - & $x$ & $x$ \\
\hline ket & - & $x$ & $\mathrm{x}$ & - & $\mathrm{x}$ \\
\hline clo & - & $x$ & $x$ & - & $\mathrm{x}$ \\
\hline \multirow[t]{2}{*}{ pak } & $x$ & $x$ & $\mathrm{x}$ & $x$ & $\mathrm{x}$ \\
\hline & - & $x$ & $x$ & - & - \\
\hline \multirow[t]{2}{*}{ ccan } & $x$ & $x$ & $x$ & $x$ & $\mathrm{x}$ \\
\hline & $?$ & $?$ & $x$ & $?$ & - \\
\hline fur & $x$ & - & - & - & - \\
\hline typ & $x$ & $x$ & $x$ & - & - \\
\hline vio & $\mathrm{x}$ & $x$ & $x$ & $x$ & $\mathrm{x}$ \\
\hline ccen & $\mathrm{x}$ & $\mathrm{x}$ & $\mathrm{x}$ & $\mathrm{x}$ & $\mathrm{X}$ \\
\hline chi & $?$ & $x$ & $x$ & - & $x$ \\
\hline arc & $\mathrm{x}$ & $\mathrm{x}$ & $\mathrm{x}$ & $\mathrm{x}$ & $\mathrm{x}$ \\
\hline urc & $\mathrm{x}$ & $\mathrm{x}$ & $\mathrm{x}$ & $\mathrm{x}$ & $\mathrm{x}$ \\
\hline \multirow[t]{2}{*}{ mcla } & $\mathrm{x}$ & $\mathrm{x}$ & $\mathrm{x}$ & $\mathrm{X}$ & $\mathrm{X}$ \\
\hline & $?$ & $?$ & - & $?$ & $\mathrm{x}$ \\
\hline
\end{tabular}


Disseta palumboi

Euaetideus giesbrechti

Euaugaptilus hecticus

E. magnus

Eucalanus nr. attenuatus

E. elongatus

E. nr. monachus

$\begin{array}{ll}\text { ege } & ? \\ & \text { x } \\ & ? \\ \text { att(s) } & \text { x } \\ \text { elg } & \text { x } \\ \text { mon } & ? \\ & - \\ & - \\ \text { inc } & - \\ \text { mar } & \text { x } \\ \text { med } & - \\ & - \\ & - \\ & - \\ & - \\ & \text { x }\end{array}$

Euchaeta barbata

E. bisinuata

$E$. hanseni

E. incisa

E. marina

E. media

$E$. norvegica

E. paraconcinna

E. pseudotonsa

E. pubera

E. spinosa

E. tonsa

* Euchaeta spp.

Euchirella amoena

E. bitumida

E. curticauda

$E$. intermedia

E. messinensis

E. rostrata

* Euchirella spp. copepodites

nr. Farrania oblonga

Gaetanus kruppi

$G$. miles

G. minor

$G$. pileatus

euk

Gaidius tenuispinus

Haloptilus longicornis

\begin{tabular}{llllll} 
euk & $\mathrm{x}$ & - & - & - & $\mathrm{x}$ \\
& $?$ & $?$ & - & $\mathrm{x}$ & - \\
& $?$ & $\mathrm{x}$ & - & $\mathrm{x}$ & - \\
\multirow{3}{*}{ eme } & $?$ & $\mathrm{x}$ & - & $?$ & $\mathrm{x}$ \\
ros & $?$ & $\mathrm{x}$ & - & $?$ & $\mathrm{x}$ \\
cuch & $?$ & $\mathrm{x}$ & $\mathrm{x}$ & $\mathrm{x}$ & $\mathrm{x}$ \\
& $?$ & $?$ & - & - & $\mathrm{x}$ \\
& $?$ & $?$ & $\mathrm{x}$ & $\mathrm{x}$ & $\mathrm{x}$ \\
& $?$ & $\mathrm{x}$ & - & $\mathrm{x}$ & $\mathrm{x}$ \\
gmi & $?$ & $\mathrm{x}$ & $\mathrm{x}$ & - & - \\
& $?$ & $\mathrm{x}$ & $\mathrm{x}$ & - & - \\
& $?$ & $\mathrm{x}$ & $\mathrm{x}$ & $?$ & $\mathrm{x}$ \\
hal & $?$ & $?$ & $\mathrm{x}$ & $?$ & - \\
orn & $?$ & $\mathrm{x}$ & $\mathrm{x}$ & $\mathrm{x}$ & $\mathrm{x}$ \\
& $?$ & - & $\mathrm{x}$ & - & - \\
& $?$ & $?$ & $\mathrm{x}$ & $?$ & - \\
& $?$ & $?$ & $\mathrm{x}$ & $?$ & $\mathrm{x}$ \\
& $?$ & $?$ & $\mathrm{x}$ & - & - \\
pap & - & - & $\mathrm{x}$ & - & - \\
spi & - & $\mathrm{x}$ & $\mathrm{x}$ & $\mathrm{x}$ & $\mathrm{x}$ \\
vip & - & $\mathrm{x}$ & $\mathrm{x}$ & $\mathrm{x}$ & $\mathrm{x}$ \\
het & - & $?$ & $\mathrm{x}$ & - & - \\
& - & $\mathrm{x}$ & $\mathrm{x}$ & - & - \\
& - & & & $?$ & -
\end{tabular}


L. latipes

Lucicutia clausi

L. ovalis

* Lucicutia spp.

Megacalanus longicornis

Metridia brevicauda

$M$. longa

M. lucens

M. princeps

$M$. venusta

* Metridia spp. copepodites

Mecynocera clausi

$\mathrm{nr}$. Microcalanus

nr. Mimocalanus

Monacilla typica

Nannocalanus minor

Neocalanus gracilis

$N$. robustior

* N. grac. \& rob. cop'tes IV,V

Pachyptilus eurygnathus

Paracandacia bispinosa

P. simplex

Paraugaptilus buchani

Phaenna spinifera

Phyllopus helgae

$P$. impar

Pleuromamma abdominalis

$P$. borealis

$P$. gracilis

$P$. piseki

$P$. robusta

P. xiphias

* P. bor. \& grac. males

* P. bor.,grac,pis. cop'dites

* P. abd.,rob.,xip. cop'dites

Pontella sp.

Pontellina plumata

nr. Pseudochirella

Racovitzanus sp.

Rhincalanus cornutus

$R$. nasutus

Scaphocalanus nr. amplius

S. nr. curtus

S. nr. longifurcus

lop ? $\mathrm{x}$

cla ? ?

? $\quad$ ?

luci,lut $\quad \mathrm{x} \quad \mathrm{x} \quad \mathrm{x} \quad \mathrm{x} \quad \mathrm{x}$

brv

lon

luc

pri

ven

cmet $\mathrm{x}$

mey $\quad \mathrm{x}$

$\operatorname{mim} \quad$ ?

mty ?

$\min \quad x$

ngr $\quad x$

nro $\quad x$

cneo $\quad \mathrm{x}$

? $\quad x$

pbi,pbs $\quad \mathrm{x}$

$\operatorname{sim} x \quad x$

psp ? $\quad$ x

hel $\quad-\quad x$

imp - $\quad x$

abd $\quad \mathrm{x} \quad \mathrm{x}$

bor $x$

gra $\quad x$

pis $\quad \mathrm{x}$

rob $\quad x$

$\begin{array}{lllll}\text { xip } & - & x & x & x\end{array}$

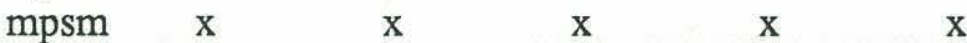

$\begin{array}{llllll}\operatorname{cpsm} & \mathrm{x} & \mathrm{x} & \mathrm{x} & \mathrm{x} & \mathrm{x}\end{array}$

$\begin{array}{llllll}\text { cplg } & x & x & x & x & x\end{array}$

pon $\begin{array}{lllll}\mathrm{x} & \mathrm{x} & \mathrm{x} & \mathrm{x} & \mathrm{x}\end{array}$

$\begin{array}{llllll} & ? & \mathrm{x} & - & ? & \mathrm{x} \\ & ? & ? & \mathrm{x} & - & - \\ \text { cor } & \mathrm{x} & \mathrm{x} & \mathrm{x} & \mathrm{x} & \mathrm{x} \\ \text { nas } & \mathrm{x} & \mathrm{x} & - & - & - \\ \text { sam } & ? & \mathrm{x} & \mathrm{x} & \mathrm{x} & ? \\ \text { scu } & ? & \mathrm{x} & \mathrm{x} & ? & \mathrm{x} \\ \text { slo } & ? & \mathrm{x} & \mathrm{x} & \mathrm{x} & \mathrm{x}\end{array}$


Scaphocalanus nr. magnus

* Scaphocalanus spp. males

Scolecithricella $n r$. abyssalis

S. ctenopus

S. dentata

S. minor

S. vittata

* Scolecithricella spp. males

Scolecithrix bradyi

S. danae

Scottocalanus nr. australis

S. helenae

S. persecans

S. securifrons

Spinocalanus $\mathrm{nr}$. spinosus

Temora longicornis/turbinata

$T$. stylifera

Temorites sp.

Undeuchaeta major

$U$. plumosa

Undinula vulgaris (cund $=\mathrm{cIV}, \mathrm{cV}$ )

$\mathrm{nr}$. Xanthocalanus agilis

nr. Xantho./Amallothrix

misc. calanoids - F,M,C

Oithona spp.

other cycl. \& harp. spp.

total copepods

\begin{tabular}{llllll} 
sma & $?$ & $?$ & $\mathrm{x}$ & $?$ & $?$ \\
mscp & $?$ & $?$ & $\mathrm{x}$ & $?$ & $\mathrm{x}$ \\
& $?$ & $?$ & $\mathrm{x}$ & $?$ & $?$ \\
\multirow{2}{*}{ den } & $?$ & $?$ & $\mathrm{x}$ & $?$ & $?$ \\
& $?$ & $?$ & $\mathrm{x}$ & $\mathrm{x}$ & $\mathrm{x}$ \\
vit & $\mathrm{x}$ & $?$ & - & $?$ & $\mathrm{x}$ \\
msca & $?$ & $?$ & $\mathrm{x}$ & - & $\mathrm{x}$ \\
bra & $\mathrm{x}$ & $\mathrm{x}$ & $\mathrm{x}$ & $?$ & $\mathrm{x}$ \\
dan & $\mathrm{x}$ & $\mathrm{x}$ & $\mathrm{x}$ & $\mathrm{x}$ & $\mathrm{x}$ \\
& $?$ & $\mathrm{x}$ & - & - & $\mathrm{x}$ \\
& $?$ & $?$ & $\mathrm{x}$ & - & - \\
& $?$ & $\mathrm{x}$ & - & - & - \\
sco & $?$ & $\mathrm{x}$ & $\mathrm{x}$ & $\mathrm{x}$ & $\mathrm{x}$ \\
spi & $?$ & $\mathrm{x}$ & $\mathrm{x}$ & $?$ & $?$ \\
ttu & $\mathrm{x}$ & $\mathrm{x}$ & - & $\mathrm{x}$ & $\mathrm{x}$ \\
tst & $\mathrm{x}$ & $\mathrm{x}$ & $\mathrm{x}$ & $\mathrm{x}$ & $\mathrm{x}$ \\
tmt & $?$ & $?$ & $\mathrm{x}$ & $?$ & - \\
umj & $?$ & $\mathrm{x}$ & $\mathrm{x}$ & $\mathrm{x}$ & $\mathrm{x}$ \\
upl & $?$ & $\mathrm{x}$ & $\mathrm{x}$ & $\mathrm{x}$ & $\mathrm{x}$ \\
und & $\mathrm{x}$ & $\mathrm{x}$ & $\mathrm{x}$ & $\mathrm{x}$ & $\mathrm{x}$ \\
& $?$ & $?$ & - & $\mathrm{x}$ & $\mathrm{x}$ \\
& $?$ & $?$ & - & $?$ & $\mathrm{x}$
\end{tabular}

misc $\quad x$

oith $\mathrm{x}$

cycl $\quad x$

totl

\# of species identified in tow

45

87

94

56

69 
Table 3: Alphabetical list of species codes, excluding the preceding $\mathrm{f}, \mathrm{m}$, and $\mathrm{c}$. Some codes do not have $\mathrm{f}, \mathrm{m}$, or $\mathrm{c}$ before the 3 letter code. These others describe a group of species within a genus or larger grouping (eg. acar = Acartia spp., totl = total copepods) and have a 4 letter code. Note: Pleuromamma piseki males $=$ mpis; $P$. gracilis and $P$. borealis males $=\mathrm{mpsm}$. These two were grouped because they are very similar in appearance while $P$. piseki is distinct.

code species name code species name

\begin{tabular}{|c|c|c|c|}
\hline aar & Aetideus armatus & hal & Haloptilus longicornis \\
\hline $\mathrm{abd}$ & Pleuromamma abdominalis & hel & Phyllopus helgae \\
\hline aca & Acrocalanus andersoni & het & Heterorhabdus spp. \\
\hline acar & Acartia spp. & hyp & Calanus hyperboreus \\
\hline $\mathrm{r}$ & Acrocalanus longicornis & imp & Phyllopus impar \\
\hline da & Acartia danae & inc & Euchaeta incisa \\
\hline rc & Clausocalanus arcuicornis & ket & Candacia ketchumi \\
\hline $\operatorname{arm}$ & Candacia armata & lam & Amallothrix laminata \\
\hline $\operatorname{tt}(\mathrm{s})$ & Eucalanus $\mathrm{nr}$. attenuatus & lon & Metridia longa \\
\hline augs & Augaptilidae spp. & lop & Lophothrix latipes \\
\hline or & Pleuromamma borealis & luc & Metridia lucens \\
\hline a & Scolecithrix bradyi & luci & Lucicutia spp. \\
\hline brv & Metridia brevicauda & lut & Lucicutia spp. \\
\hline & $\begin{array}{l}\text { Calanus, Neocalanus, Undinula } \\
\text { copepodites }=<\text { III }\end{array}$ & $\begin{array}{l}\operatorname{mar} \\
\text { med }\end{array}$ & $\begin{array}{l}\text { Euchaeta marina } \\
\text { E. media }\end{array}$ \\
\hline & Candacia spp. copepodites & met & Metridia spp. copodites \\
\hline$n$ & Centropages spp. copepodites & mey & Mecynocera clausi \\
\hline & Chirundina streetsi & $\operatorname{mim}$ & nr. Mimocalanus \\
\hline & Lucicutia clausi & $\min$ & Nannocalanus minor \\
\hline lo & Candacia longimana & misc & misc. calanoids - F,M,C \\
\hline or & Rhincalanus cornutus & mon & Eucalanus nr. monachus \\
\hline Ir & Candacia curta & mty & Monacilla typica \\
\hline $\mathrm{cl}$ & non-Oithona cyclopoids & nas & Rhincalanus nasutus \\
\hline & \& harpacticoid spp. & neg & Acartia negligens \\
\hline & Scolecithrix danae & neo & Neocalanus gracilis \& robustior \\
\hline n & Scolecithricella dentata & & stage IV + V copepodites \\
\hline & Euaetideus giesbrechti & ngr & Neocalanus gracilis \\
\hline & Eucalanus elongatus & nro & N. robustior \\
\hline eme & Euchirella messinensis & oith & Oithona spp. \\
\hline & Candacia ethiopica & orn & Haloptilus ornatus \\
\hline $\mathrm{lk}$ & Euchaeta spp. & pak & Candacia pachydactyla \\
\hline & Calanus finmarchicus & pap & Heterorhabdus papilliger \\
\hline (v)fin & stage V copepodites & pav & Calocalanus parvoninus \\
\hline (c)fin & stage IV copepodites & pbi & Paracandacia bispinosa \\
\hline & Centropages furcatus & pis & Pleuromamma piseki \\
\hline mi & Gaetanus minor & plg & P. abdominalis, P.robustior, \& \\
\hline & Pleuromamma gracilis & & P. xiphias copepodites \\
\hline
\end{tabular}




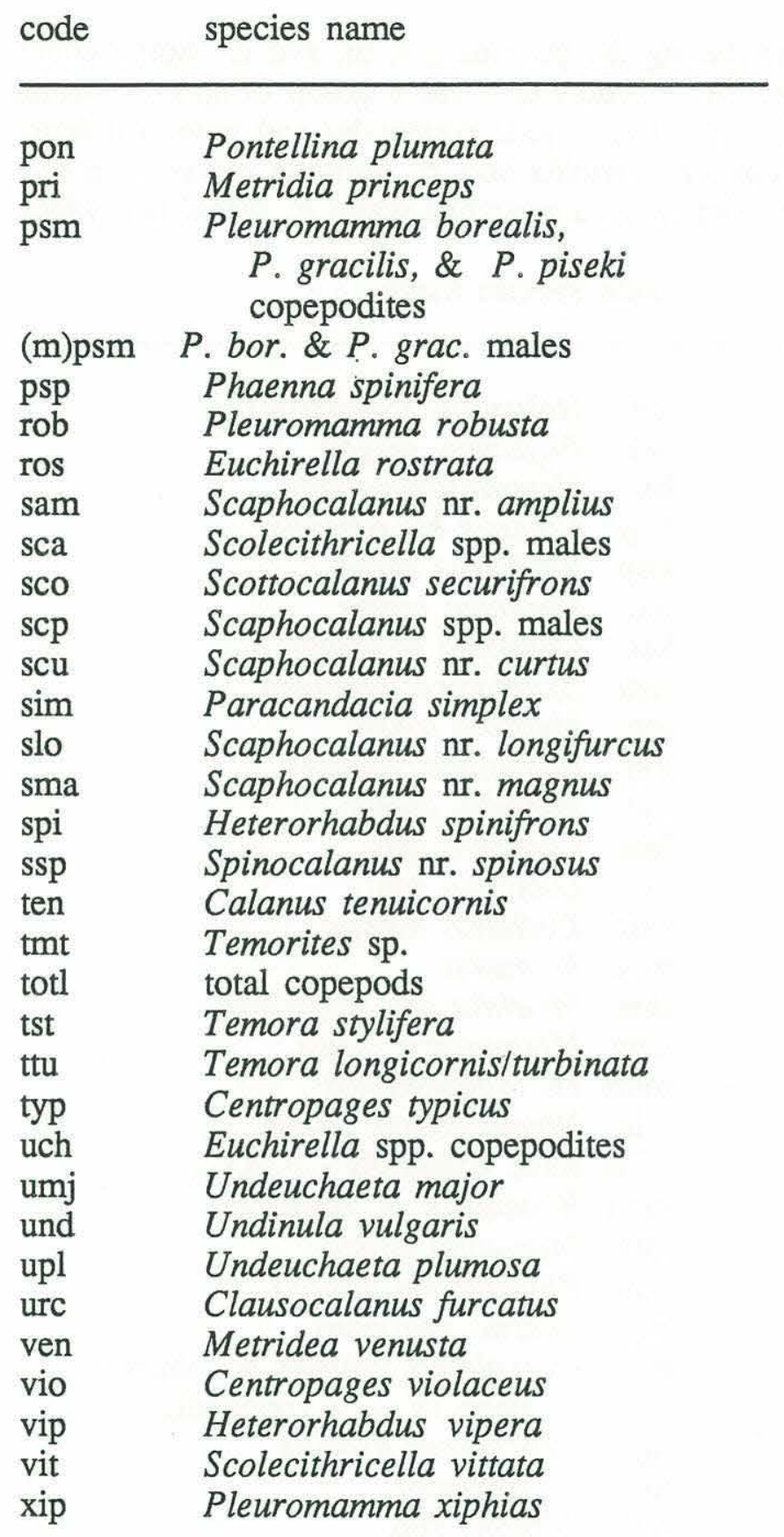


Table 4. Copepod counts from MOCNESS tows. Tow number is given at top left of each page followed by 'raw', 'std', 'int', or 'cum'. Page 2 (The Data) explains how the raw, standardized, integrated, and cumulative percent was determined.

mocness-1-cop217.raw WCR-82H MOCNESS tow copepods

$4 a-1$

\begin{tabular}{crrrrrrrrr} 
net\# & midd & depi & volf & aliq & ffin & mfin & vfin & cfin & fhyp \\
\hline 0 & 62 & 123 & 650 & 4 & 0 & 0 & 9 & 2 & 0 \\
1 & 98 & 50 & 312 & 1 & 0 & 0 & 1 & 3 & 0 \\
2 & 59 & 29 & 240 & 4 & 0 & 1 & 3 & 34 & 0 \\
3 & 22 & 44 & 336 & 2 & 0 & 0 & 2 & 3 & 0 \\
4 & 63 & 126 & 677 & 4 & 0 & 0 & 2 & 35 & 1 \\
5 & 102 & 49 & 257 & 1 & 0 & 0 & 4 & 7 & 0 \\
6 & 58 & 38 & 261 & 4 & 0 & 0 & 2 & 68 & 0 \\
7 & 20 & 39 & 262 & 2 & 0 & 0 & 0 & 1 & 0 \\
8 & 60 & 120 & 731 & 4 & 0 & 0 & 5 & 119 & 0 \\
11 & 97 & 46 & 298 & 1 & 0 & 0 & 0 & 4 & 0 \\
12 & 51 & 47 & 396 & 2 & 0 & 0 & 5 & 208 & 0 \\
13 & 14 & 27 & 217 & 2 & 0 & 0 & 12 & 21 & 0 \\
14 & 60 & 120 & 888 & 4 & 0 & 0 & 9 & 59 & 0 \\
15 & 94 & 52 & 510 & 1 & 0 & 0 & 2 & 7 & 0 \\
16 & 46 & 45 & 404 & 4 & 1 & 2 & 9 & 130 & 0 \\
17 & 12 & 23 & 454 & 8 & 1 & 0 & 0 & 2 & 0 \\
18 & 67 & 134 & 930 & 16 & 1 & 2 & 0 & 15 & 0 \\
19 & 67 & 134 & 965 & 16 & 0 & 0 & 1 & 1 & 0 \\
\hline
\end{tabular}

\begin{tabular}{|c|c|c|c|c|c|c|c|c|c|}
\hline net\# & chyp & ften & mten & cten & fngr & mngr & fnro & mnro & cneo \\
\hline 0 & 3 & 3 & 0 & 1 & 0 & 0 & 0 & 0 & 6 \\
\hline 1 & 0 & 16 & 2 & 70 & 0 & 0 & 0 & 0 & 7 \\
\hline 2 & 0 & 0 & 1 & 1 & 0 & 0 & 1 & 1 & 1 \\
\hline 3 & 0 & 0 & 0 & 1 & 0 & 0 & 0 & 0 & 0 \\
\hline 4 & 0 & 3 & 0 & 5 & 0 & 1 & 0 & 0 & 0 \\
\hline 5 & 1 & 19 & 2 & 59 & 0 & 0 & 0 & 0 & 13 \\
\hline 6 & 0 & 0 & 0 & 1 & 1 & 0 & 2 & 0 & 1 \\
\hline 7 & 0 & 0 & 0 & 0 & 0 & 0 & 0 & 0 & 0 \\
\hline 8 & 0 & 4 & 1 & 11 & 0 & 0 & 1 & 0 & 3 \\
\hline 11 & 0 & 13 & 0 & 50 & 0 & 0 & 0 & 0 & 6 \\
\hline 12 & 0 & 2 & 0 & 6 & 0 & 0 & 1 & 0 & 6 \\
\hline 13 & 0 & 0 & 0 & 0 & 0 & 0 & 1 & 0 & 0 \\
\hline 14 & 0 & 4 & 0 & 12 & 0 & 0 & 0 & 0 & 2 \\
\hline 15 & 0 & 37 & 3 & 139 & 0 & 0 & 0 & 0 & 13 \\
\hline 16 & 0 & 0 & 0 & 12 & 0 & 0 & 0 & 0 & 1 \\
\hline 17 & 0 & 0 & 0 & 0 & 0 & 0 & 0 & 0 & 1 \\
\hline 18 & 0 & 4 & 0 & 6 & 0 & 0 & 0 & 0 & 1 \\
\hline 19 & 0 & 5 & 0 & 61 & 0 & 0 & 0 & 0 & 1 \\
\hline
\end{tabular}


mocness-1-cop217.raw WCR-82H MOCNESS tow copepods

$4 a=2$

\begin{tabular}{|c|c|c|c|c|c|c|c|c|c|}
\hline net\# & fund & mund & vund & cund & ccal & fmin & mmin & cmin & felg \\
\hline 0 & 33 & 29 & 36 & 6 & 4 & 29 & 28 & 231 & 0 \\
\hline 1 & 0 & 0 & 0 & 0 & 58 & 9 & 9 & 55 & 0 \\
\hline 2 & 143 & 77 & 102 & 11 & 11 & 116 & 57 & 202 & 1 \\
\hline 3 & 7 & 1 & 20 & 26 & 0 & 7 & 3 & 31 & 0 \\
\hline 4 & 38 & 14 & 31 & 12 & 12 & 49 & 19 & 118 & 0 \\
\hline 5 & 2 & 1 & 0 & 1 & 54 & 30 & 10 & 55 & 0 \\
\hline 6 & 42 & 38 & 13 & 9 & 13 & 47 & 17 & 175 & 0 \\
\hline 7 & 2 & 1 & 10 & 7 & 2 & 3 & 1 & 9 & 0 \\
\hline 8 & 7 & 4 & 10 & 5 & 32 & 44 & 17 & 191 & 2 \\
\hline 11 & 0 & 3 & 5 & 1 & 62 & 40 & 18 & 180 & 1 \\
\hline 12 & 2 & 1 & 0 & 0 & 64 & 92 & 23 & 330 & 6 \\
\hline 13 & 2 & 2 & 0 & 0 & 1 & 13 & 0 & 13 & 0 \\
\hline 14 & 0 & 0 & 0 & 0 & 8 & 43 & 14 & 126 & 0 \\
\hline 15 & 0 & 0 & 0 & 0 & 48 & 130 & 67 & 96 & 1 \\
\hline 16 & 1 & 0 & 0 & 0 & 52 & 55 & 36 & 123 & 3 \\
\hline 17 & 1 & 1 & 0 & 0 & 2 & 48 & 34 & 184 & 0 \\
\hline 18 & 0 & 0 & 0 & 0 & 18 & 60 & 27 & 189 & 0 \\
\hline 19 & 0 & 1 & 1 & 0 & 112 & 59 & 50 & 456 & 0 \\
\hline
\end{tabular}

\begin{tabular}{|c|c|c|c|c|c|c|c|c|c|}
\hline net\# & celg & fcor & mcor & ccor & fnas & cnas & fmey & fpav & farc \\
\hline 0 & 0 & 0 & 0 & 1 & 0 & 0 & 0 & 22 & 6 \\
\hline 1 & 0 & 2 & 0 & 4 & 0 & 0 & 1 & 1 & 6 \\
\hline 2 & 0 & 0 & 0 & 0 & 0 & 1 & 0 & 5 & 3 \\
\hline 3 & 0 & 0 & 0 & 0 & 0 & 0 & 3 & 13 & 7 \\
\hline 4 & 0 & 0 & 0 & 5 & 0 & 1 & 0 & 6 & 8 \\
\hline 5 & 3 & 4 & 0 & 16 & 0 & 1 & 0 & 1 & 4 \\
\hline 6 & 1 & 0 & 0 & 0 & 0 & 4 & 1 & 1 & 3 \\
\hline 7 & 0 & 0 & 0 & 1 & 0 & 0 & 0 & 3 & 21 \\
\hline 8 & 3 & 1 & 1 & 9 & 0 & 1 & 2 & 6 & 28 \\
\hline 11 & 2 & 0 & 0 & 20 & 0 & 2 & 0 & 2 & 17 \\
\hline 12 & 15 & 0 & 0 & 7 & 0 & 12 & 1 & 0 & 8 \\
\hline 13 & 1 & 0 & 0 & 1 & 0 & 0 & 0 & 1 & 66 \\
\hline 14 & 3 & 2 & 0 & 10 & 0 & 5 & 0 & 3 & 25 \\
\hline 15 & 8 & 7 & 1 & 32 & 1 & 1 & 0 & 0 & 59 \\
\hline 16 & 23 & 0 & 0 & 12 & 0 & 9 & 0 & 0 & 3 \\
\hline 17 & 0 & 2 & 0 & 11 & 0 & 0 & 0 & 3 & 106 \\
\hline 18 & 4 & 0 & 0 & 7 & 0 & 0 & 0 & 5 & 87 \\
\hline 19 & 1 & 1 & 0 & 9 & 0 & 2 & 15 & 4 & 41 \\
\hline
\end{tabular}


mocness-1-cop217.raw WCR-82H MOCNESS tow copepods

$4 a-3$

\begin{tabular}{crrrrrrrrr} 
net\# & fmar & mmar & ceuk & fbra & mbra & fdan & mdan & ftst & mtst \\
\hline 0 & 50 & 15 & 201 & 1 & 0 & 91 & 52 & 4 & 2 \\
1 & 0 & 0 & 34 & 4 & 1 & 1 & 0 & 0 & 0 \\
2 & 67 & 20 & 95 & 0 & 0 & 101 & 57 & 3 & 1 \\
3 & 86 & 33 & 71 & 0 & 0 & 152 & 77 & 8 & 6 \\
4 & 0 & 1 & 20 & 0 & 0 & 36 & 8 & 2 & 0 \\
5 & 1 & 0 & 41 & 3 & 0 & 12 & 5 & 0 & 0 \\
6 & 0 & 0 & 22 & 0 & 0 & 13 & 7 & 1 & 1 \\
7 & 9 & 3 & 8 & 0 & 0 & 8 & 1 & 1 & 0 \\
8 & 6 & 1 & 58 & 0 & 1 & 21 & 7 & 2 & 0 \\
11 & 1 & 0 & 60 & 3 & 0 & 11 & 1 & 0 & 0 \\
12 & 3 & 1 & 139 & 0 & 0 & 20 & 13 & 1 & 0 \\
13 & 7 & 0 & 2 & 0 & 0 & 3 & 2 & 0 & 0 \\
14 & 2 & 0 & 8 & 1 & 0 & 20 & 4 & 1 & 0 \\
15 & 0 & 0 & 112 & 8 & 0 & 6 & 0 & 0 & 0 \\
16 & 2 & 0 & 10 & 0 & 0 & 20 & 11 & 0 & 0 \\
17 & 4 & 0 & 7 & 0 & 0 & 10 & 7 & 1 & 0 \\
18 & 3 & 2 & 10 & 0 & 0 & 11 & 2 & 0 & 0 \\
19 & 0 & 0 & 0 & 2 & 2 & 18 & 6 & 0 & 0 \\
\hline
\end{tabular}

\begin{tabular}{|c|c|c|c|c|c|c|c|c|c|}
\hline net\# & ftur & mtur & fluc & mluc & cluc & fbor & fgra & mpsm & mpis \\
\hline 0 & 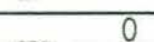 & 0 & 1 & 1 & 12 & 0 & 0 & 0 & 0 \\
\hline 1 & 0 & 0 & 2 & 0 & 2 & 0 & 0 & 0 & 1 \\
\hline 2 & 8 & 0 & 0 & 0 & 0 & 0 & 0 & 0 & 0 \\
\hline 3 & 15 & 2 & 0 & 2 & 1 & 0 & 0 & 0 & 0 \\
\hline 4 & 12 & 6 & 0 & 0 & 0 & 0 & 0 & 0 & 0 \\
\hline 5 & 1 & 0 & 1 & 0 & 1 & 1 & 1 & 0 & 0 \\
\hline 6 & 13 & 0 & 0 & 0 & 0 & 0 & 0 & 0 & 0 \\
\hline 7 & 6 & 5 & 0 & 0 & 0 & 1 & 0 & 0 & 0 \\
\hline 8 & 3 & 1 & 0 & 0 & 2 & 0 & 0 & 0 & 0 \\
\hline 11 & 1 & 0 & 0 & 0 & 1 & 0 & 6 & 4 & 0 \\
\hline 12 & 2 & 0 & 0 & 0 & 0 & 0 & 0 & 0 & 0 \\
\hline 13 & 0 & 0 & 0 & 0 & 0 & 0 & 0 & 0 & 0 \\
\hline 14 & 2 & 0 & 0 & 0 & 0 & 0 & 0 & 0 & 0 \\
\hline 15 & 0 & 0 & 0 & 0 & 0 & 0 & 0 & 1 & 0 \\
\hline 16 & 0 & 0 & 0 & 0 & 0 & 0 & 0 & 0 & 0 \\
\hline 17 & 0 & 0 & 0 & 0 & 0 & 0 & 0 & 0 & 0 \\
\hline 18 & 0 & 0 & 0 & 0 & 0 & 0 & 0 & 0 & 0 \\
\hline 19 & 0 & 0 & 0 & 0 & 30 & 0 & 0 & 0 & 0 \\
\hline
\end{tabular}


mocness-1-cop217.raw WCR-82H MOCNESS tow copepods

$4 a-4$

\begin{tabular}{|c|c|c|c|c|c|c|c|c|c|}
\hline net\# & cpsm & mabd & mrob & cplg & ftyp & mtyp & ffur & mfur & ccen \\
\hline 0 & 0 & 0 & 1 & 2 & 0 & 1 & 2 & 2 & 3 \\
\hline 1 & 0 : & 0 & 0 & 3 & 0 & 1 & 0 & 1 & 1 \\
\hline 2 & 0. & 0 & 0 & 0 & 0 & 0 & 0 & 0 & 0 \\
\hline 3 & 0 & 0 & 0 & 0 & 5 & 1 & 5 & 2 & 4 \\
\hline 4 & 0 & 0 & 0 & 0 & 6 & 1 & 2 & 4 & -999 \\
\hline 5 & 0 & 0 & 0 & 3 & 3 & 3 & 0 & 0 & 2 \\
\hline 6 & 0 & 0 & 0 & 0 & 2 & 0 & 1 & 0 & 5 \\
\hline 7 & 0 & 0 & 0 & 1 & 74 & 13 & 0 & 1 & 1 \\
\hline 8 & 0 & 1 & 0 & 2 & 61 & 31 & 1 & 1 & 23 \\
\hline 11 & 7 & 0 & 0 & 1 & 2 & 1 & 0 & 0 & 1 \\
\hline 12 & 0 & 0 & 0 & 0 & 22 & 11 & 0 & 0 & 20 \\
\hline 13 & 0 & 0 & 0 & 0 & 142 & 68 & 0 & 0 & 58 \\
\hline 14 & 1 & 0 & 0 & 0 & 105 & 52 & 0 & 0 & 133 \\
\hline 15 & 0 & 0 & 0 & 5 & 4 & 2 & 0 & 0 & 1 \\
\hline 16 & 0 & 0 & 0 & 0 & 12 & 16 & 0 & 0 & 28 \\
\hline 17 & 0 & 0 & 0 & 0 & 408 & 271 & 0 & 0 & 327 \\
\hline 18 & 0 & 0 & 0 & 6 & 217 & 136 & 0 & 0 & 255 \\
\hline 19 & 1 & 0 & 0 & 20 & 361 & 286 & 1 & 2 & 507 \\
\hline
\end{tabular}

\begin{tabular}{crrrrrrrrr} 
net\# & fvio & mvio & cvio & luci & fhal & chal & farm & marm & fbip \\
\hline 0 & 1 & 2 & 0 & 36 & 0 & 1 & 0 & 0 & 0 \\
1 & 3 & 0 & 0 & 239 & 19 & 26 & 0 & 0 & 0 \\
2 & 0 & 0 & 1 & 4 & 0 & 0 & 0 & 0 & 0 \\
3 & 7 & 5 & 6 & 1 & 0 & 0 & 0 & 0 & 0 \\
4 & 1 & 1 & 2 & 21 & 0 & 2 & 0 & 0 & 0 \\
5 & 3 & 0 & 0 & 133 & 11 & 14 & 0 & 0 & 0 \\
6 & 0 & 0 & 0 & 19 & 0 & 0 & 0 & 0 & 0 \\
7 & 21 & 10 & 10 & 0 & 0 & 0 & 0 & 0 & 0 \\
8 & 19 & 5 & 13 & 23 & 4 & 2 & 0 & 0 & 0 \\
11 & 3 & 0 & 0 & 118 & 6 & 8 & 0 & 0 & 0 \\
12 & 7 & 6 & 13 & 11 & 0 & 0 & 1 & 0 & 0 \\
13 & 27 & 31 & 10 & 0 & 0 & 0 & 4 & 2 & 0 \\
14 & 20 & 10 & 34 & 58 & 4 & 3 & 1 & 1 & 0 \\
15 & 5 & 4 & 0 & 386 & 53 & 8 & 0 & 0 & 0 \\
16 & 4 & 8 & 4 & 6 & 0 & 0 & 0 & 1 & 0 \\
17 & 26 & 19 & 6 & 2 & 0 & 0 & 4 & 1 & 1 \\
18 & 20 & 9 & 8 & 11 & 2 & 3 & 2 & 2 & 0 \\
19 & 58 & 48 & 41 & 71 & 6 & 15 & 3 & 4 & 0 \\
\hline
\end{tabular}


mocness-1-cop217. raw WCR-82H MOCNESS tow copepods

Ha 5

net\# fpak mpak fpbi mpbi fsim msim ccan fpon mpon

\begin{tabular}{rrrllrlrll}
\hline 0 & 20 & 24 & 1 & 0 & 3 & 0 & 143 & 1 & 2 \\
1 & 1 & 1 & 1 & 0 & 0 & 1 & 4 & 0 & 0 \\
2 & 4 & 2 & 0 & 0 & 0 & 3 & 6 & 0 & 1 \\
3 & 4 & 5 & 1 & 0 & 11 & 6 & 9 & 2 & 2 \\
4 & 2 & 4 & 0 & 0 & 0 & 0 & 12 & 3 & 0 \\
5 & 1 & 0 & 0 & 0 & 0 & 0 & 3 & 0 & 0 \\
6 & 0 & 0 & 0 & 0 & 0 & 0 & 1 & 0 & 0 \\
7 & 14 & 18 & 0 & 2 & 0 & 0 & 35 & 3 & 0 \\
8 & 7 & 8 & 0 & 0 & 2 & 1 & 7 & 2 & 0 \\
11 & 1 & 0 & 1 & 1 & 0 & 0 & 7 & 0 & 0 \\
12 & 0 & 0 & 0 & 0 & 0 & 0 & 3 & 0 & 0 \\
13 & 0 & 0 & 0 & 0 & 0 & 0 & 2 & 0 & 0 \\
14 & 0 & 0 & 0 & 0 & 0 & 0 & 4 & 0 & 0 \\
15 & 1 & 0 & 1 & 0 & 0 & 0 & 4 & 0 & 0 \\
16 & 0 & 0 & 0 & 0 & 0 & 0 & 2 & 0 & 0 \\
17 & 0 & 0 & 0 & 0 & 1 & 0 & 9 & 1 & 0 \\
18 & 0 & 0 & 0 & 0 & 1 & 0 & 8 & 0 & 0 \\
19 & 1 & 0 & 0 & 0 & 0 & 0 & 0 & 0 & 0 \\
\hline
\end{tabular}

net\# acar fmis mis cmis oith cycl totl

\begin{tabular}{rrrrrrrr}
\hline 0 & 7 & 36 & 11 & 187 & 17 & 824 & 2206 \\
1 & 0 & 70 & 11 & 472 & 35 & 104 & 1282 \\
2 & 110 & 0 & 6 & 78 & 9 & 106 & 1454 \\
3 & 9 & 32 & 0 & 171 & 1 & 586 & 1452 \\
4 & 3 & 37 & 7 & 100 & 14 & 231 & 898 \\
5 & 0 & 24 & 10 & 370 & 11 & 70 & 1013 \\
6 & 30 & 11 & 7 & 29 & 10 & 36 & 647 \\
7 & 10 & 18 & 6 & 55 & 4 & 198 & 597 \\
8 & 49 & 30 & 10 & 129 & 43 & 164 & 1247 \\
11 & 1 & 35 & 21 & 336 & 106 & 82 & 1254 \\
12 & 198 & 24 & 6 & 105 & 203 & 89 & 1687 \\
13 & 16 & 10 & 12 & 49 & 1 & 31 & 611 \\
14 & 144 & 47 & 9 & 148 & 80 & 87 & 1304 \\
15 & 2 & 35 & 23 & 712 & 47 & 109 & 2181 \\
16 & 5 & 22 & 1 & 73 & 83 & 14 & 794 \\
17 & 13 & 55 & 36 & 259 & 0 & 48 & 1912 \\
18 & 22 & 30 & 25 & 202 & 13 & 45 & 1471 \\
19 & 86 & 145 & 56 & 516 & 195 & 94 & 3396 \\
\hline
\end{tabular}


mocness-1-cop217.std WCR-82H MOCNESS tow copepods

$4 b-1$

\begin{tabular}{crrrrrrrrr} 
net\# & midd & depi & volf & aliq & \#ffin & \#mfin & \#vfin & \#cfin & \#fhyp \\
\hline 0 & & & & & & & & & \\
1 & 98.5 & 123.0 & 650.2 & 4.0 & 0.0 & 0.0 & 55.4 & 12.3 & 0.0 \\
2 & 58.5 & 29.0 & 312.4 & 1.0 & 0.0 & 0.0 & 3.2 & 9.6 & 0.0 \\
3 & 22.0 & 44.0 & 335.7 & 4.0 & 0.0 & 16.7 & 50.1 & 567.4 & 0.0 \\
4 & 63.0 & 126.0 & 677.2 & 2.0 & 0.0 & 0.0 & 11.9 & 17.9 & 0.0 \\
5 & 101.5 & 49.0 & 256.6 & 1.0 & 0.0 & 0.0 & 11.8 & 206.7 & 5.9 \\
6 & 58.0 & 38.0 & 260.6 & 4.0 & 0.0 & 0.0 & 15.6 & 27.3 & 0.0 \\
7 & 19.5 & 39.0 & 261.5 & 1.6 & 0.0 & 0.0 & 30.7 & 1043.7 & 0.0 \\
8 & 60.0 & 120.0 & 731.3 & 4.0 & 0.0 & 0.0 & 27.3 & 650.9 & 0.0 \\
11 & 97.0 & 46.0 & 297.5 & 1.0 & 0.0 & 0.0 & 0.0 & 13.4 & 0.0 \\
12 & 50.5 & 47.0 & 396.4 & 2.0 & 0.0 & 0.0 & 25.2 & 1049.4 & 0.0 \\
13 & 13.5 & 27.0 & 216.7 & 2.0 & 0.0 & 0.0 & 110.8 & 193.8 & 0.0 \\
14 & 60.0 & 120.0 & 887.6 & 4.0 & 0.0 & 0.0 & 40.6 & 265.9 & 0.0 \\
15 & 94.0 & 52.0 & 509.7 & 1.0 & 0.0 & 0.0 & 3.9 & 13.7 & 0.0 \\
16 & 45.5 & 45.0 & 404.1 & 4.0 & 9.9 & 19.8 & 89.1 & 1286.8 & 0.0 \\
17 & 11.5 & 23.0 & 454.3 & 8.0 & 17.6 & 0.0 & 0.0 & 35.2 & 0.0 \\
18 & 67.0 & 134.0 & 929.6 & 16.0 & 17.2 & 34.4 & 0.0 & 258.2 & 0.0 \\
19 & 67.0 & 134.0 & 965.3 & 16.0 & 0.0 & 0.0 & 16.6 & 16.6 & 0.0 \\
\hline
\end{tabular}

\begin{tabular}{crrrrrrrrr} 
net\# & \#chyp & \#ften & \#mten & \#cten & \#fngr & \#mngr & \#fnro & \#mnro & \#cneo \\
\hline 0 & 18.5 & 18.5 & 0.0 & 6.2 & 0.0 & 0.0 & 0.0 & 0.0 & 36.9 \\
1 & 0.0 & 51.2 & 6.4 & 224.1 & 0.0 & 0.0 & 0.0 & 0.0 & 22.4 \\
2 & 0.0 & 0.0 & 16.7 & 16.7 & 0.0 & 0.0 & 16.7 & 16.7 & 16.7 \\
3 & 0.0 & 0.0 & 0.0 & 6.0 & 0.0 & 0.0 & 0.0 & 0.0 & 0.0 \\
4 & 0.0 & 17.7 & 0.0 & 29.5 & 0.0 & 5.9 & 0.0 & 0.0 & 0.0 \\
5 & 3.9 & 74.0 & 7.8 & 229.9 & 0.0 & 0.0 & 0.0 & 0.0 & 50.7 \\
6 & 0.0 & 0.0 & 0.0 & 15.3 & 15.3 & 0.0 & 30.7 & 0.0 & 15.3 \\
7 & 0.0 & 0.0 & 0.0 & 0.0 & 0.0 & 0.0 & 0.0 & 0.0 & 0.0 \\
8 & 0.0 & 21.9 & 5.5 & 60.2 & 0.0 & 0.0 & 5.5 & 0.0 & 16.4 \\
11 & 0.0 & 43.7 & 0.0 & 168.1 & 0.0 & 0.0 & 0.0 & 0.0 & 20.2 \\
12 & 0.0 & 10.1 & 0.0 & 30.3 & 0.0 & 0.0 & 5.0 & 0.0 & 30.3 \\
13 & 0.0 & 0.0 & 0.0 & 0.0 & 0.0 & 0.0 & 9.2 & 0.0 & 0.0 \\
14 & 0.0 & 18.0 & 0.0 & 54.1 & 0.0 & 0.0 & 0.0 & 0.0 & 9.0 \\
15 & 0.0 & 72.6 & 5.9 & 272.7 & 0.0 & 0.0 & 0.0 & 0.0 & 25.5 \\
16 & 0.0 & 0.0 & 0.0 & 118.8 & 0.0 & 0.0 & 0.0 & 0.0 & 9.9 \\
17 & 0.0 & 0.0 & 0.0 & 0.0 & 0.0 & 0.0 & 0.0 & 0.0 & 17.6 \\
18 & 0.0 & 68.8 & 0.0 & 103.3 & 0.0 & 0.0 & 0.0 & 0.0 & 17.2 \\
19 & 0.0 & 82.9 & 0.0 & 1011.1 & 0.0 & 0.0 & 0.0 & 0.0 & 16.6 \\
& & & & & & & & & \\
\hline
\end{tabular}


mocness-1-cop217.std WCR-82H MOCNESS tow copepods

$4 b-2$

net\# \#fund \#mund \#vund \#cund \#ccal \#fmin \#mmin \#cmin \#felg

\begin{tabular}{|c|c|c|c|c|c|c|c|c|c|}
\hline $\begin{array}{r}0 \\
1 \\
2 \\
3 \\
4 \\
5 \\
6 \\
7 \\
8 \\
11 \\
12 \\
13 \\
14 \\
15 \\
16 \\
17 \\
18 \\
19\end{array}$ & $\begin{array}{r}203.0 \\
0.0 \\
2386.3 \\
41.7 \\
224.5 \\
7.8 \\
644.7 \\
12.2 \\
38.3 \\
0.0 \\
10.1 \\
18.5 \\
0.0 \\
0.0 \\
9.9 \\
17.6 \\
0.0 \\
0.0\end{array}$ & $\begin{array}{r}178.4 \\
0.0 \\
1284.9 \\
6.0 \\
82.7 \\
3.9 \\
583.3 \\
6.1 \\
21.9 \\
10.1 \\
5.0 \\
18.5 \\
0.0 \\
0.0 \\
0.0 \\
17.6 \\
0.0 \\
16.6\end{array}$ & $\begin{array}{r}221.5 \\
0.0 \\
1702.1 \\
119.1 \\
183.1 \\
0.0 \\
199.5 \\
61.2 \\
54.7 \\
16.8 \\
0.0 \\
0.0 \\
0.0 \\
0.0 \\
0.0 \\
0.0 \\
0.0 \\
16.6\end{array}$ & $\begin{array}{r}36.9 \\
0.0 \\
183.6 \\
154.8 \\
70.9 \\
3.9 \\
138.1 \\
42.8 \\
27.3 \\
3.4 \\
0.0 \\
0.0 \\
0.0 \\
0.0 \\
0.0 \\
0.0 \\
0.0 \\
0.0\end{array}$ & $\begin{array}{r}24.6 \\
185.7 \\
183.6 \\
0.0 \\
70.9 \\
210.4 \\
199.5 \\
12.2 \\
175.0 \\
208.4 \\
322.9 \\
9.2 \\
36.1 \\
94.2 \\
514.7 \\
35.2 \\
309.8 \\
1856.4\end{array}$ & $\begin{array}{r}178.4 \\
28.8 \\
1935.8 \\
41.7 \\
289.4 \\
116.9 \\
721.4 \\
18.4 \\
240.7 \\
134.5 \\
464.2 \\
120.0 \\
193.8 \\
255.1 \\
544.4 \\
845.3 \\
1032.7 \\
977.9\end{array}$ & $\begin{array}{r}172.3 \\
28.8 \\
951.2 \\
17.9 \\
112.2 \\
39.0 \\
260.9 \\
6.1 \\
93.0 \\
60.5 \\
116.0 \\
0.0 \\
63.1 \\
131.4 \\
356.3 \\
598.7 \\
464.7 \\
828.8\end{array}$ & $\begin{array}{r}1421.1 \\
176.1 \\
3370.9 \\
184.6 \\
697.0 \\
214.3 \\
2686.1 \\
55.1 \\
1044.7 \\
605.0 \\
1665.0 \\
120.0 \\
567.8 \\
188.3 \\
1217.5 \\
3240.1 \\
3253.0 \\
7558.3\end{array}$ & $\begin{array}{r}0.0 \\
0.0 \\
16.7 \\
0.0 \\
0.0 \\
0.0 \\
0.0 \\
0.0 \\
10.9 \\
3.4 \\
30.3 \\
0.0 \\
0.0 \\
2.0 \\
29.7 \\
0.0 \\
0.0 \\
0.0\end{array}$ \\
\hline
\end{tabular}

net\# \#celg \#fcor \#mcor \#ccor \#fnas \#cnas \#fmey \#fpav \#farc

\begin{tabular}{rrrrrrrrrr}
\hline 0 & 0.0 & 0.0 & 0.0 & 6.2 & 0.0 & 0.0 & 0.0 & 135.3 & 36.9 \\
1 & 0.0 & 6.4 & 0.0 & 12.8 & 0.0 & 0.0 & 3.2 & 3.2 & 19.2 \\
2 & 0.0 & 0.0 & 0.0 & 0.0 & 0.0 & 16.7 & 0.0 & 83.4 & 50.1 \\
3 & 0.0 & 0.0 & 0.0 & 0.0 & 0.0 & 0.0 & 17.9 & 77.4 & 41.7 \\
4 & 0.0 & 0.0 & 0.0 & 29.5 & 0.0 & 5.9 & 0.0 & 35.4 & 47.3 \\
5 & 11.7 & 15.6 & 0.0 & 62.4 & 0.0 & 3.9 & 0.0 & 3.9 & 15.6 \\
6 & 15.3 & 0.0 & 0.0 & 0.0 & 0.0 & 61.4 & 15.3 & 15.3 & 46.0 \\
7 & 0.0 & 0.0 & 0.0 & 6.1 & 0.0 & 0.0 & 0.0 & 18.4 & 128.5 \\
8 & 16.4 & 5.5 & 5.5 & 49.2 & 0.0 & 5.5 & 10.9 & 32.8 & 153.2 \\
11 & 6.7 & 0.0 & 0.0 & 67.2 & 0.0 & 6.7 & 0.0 & 6.7 & 57.1 \\
12 & 75.7 & 0.0 & 0.0 & 35.3 & 0.0 & 60.5 & 5.0 & 0.0 & 40.4 \\
13 & 9.2 & 0.0 & 0.0 & 9.2 & 0.0 & 0.0 & 0.0 & 9.2 & 609.1 \\
14 & 13.5 & 9.0 & 0.0 & 45.1 & 0.0 & 22.5 & 0.0 & 13.5 & 112.7 \\
15 & 15.7 & 13.7 & 2.0 & 62.8 & 2.0 & 2.0 & 0.0 & 0.0 & 115.8 \\
16 & 227.7 & 0.0 & 0.0 & 118.8 & 0.0 & 89.1 & 0.0 & 0.0 & 29.7 \\
17 & 0.0 & 35.2 & 0.0 & 193.7 & 0.0 & 0.0 & 0.0 & 52.8 & 1866.6 \\
18 & 68.8 & 0.0 & 0.0 & 120.5 & 0.0 & 0.0 & 0.0 & 86.1 & 1497.4 \\
19 & 16.6 & 16.6 & 0.0 & 149.2 & 0.0 & 33.2 & 248.6 & 66.3 & 679.6 \\
& & & & & & & & & \\
\hline
\end{tabular}


mocness-1-cop217.std WCR-82H MOCNESS tow copepods

$4 b-3$

net\# \#fmar \#mmar \#ceuk \#fbra \#mbra \#fdan \#mdan \#ftst \#mtst

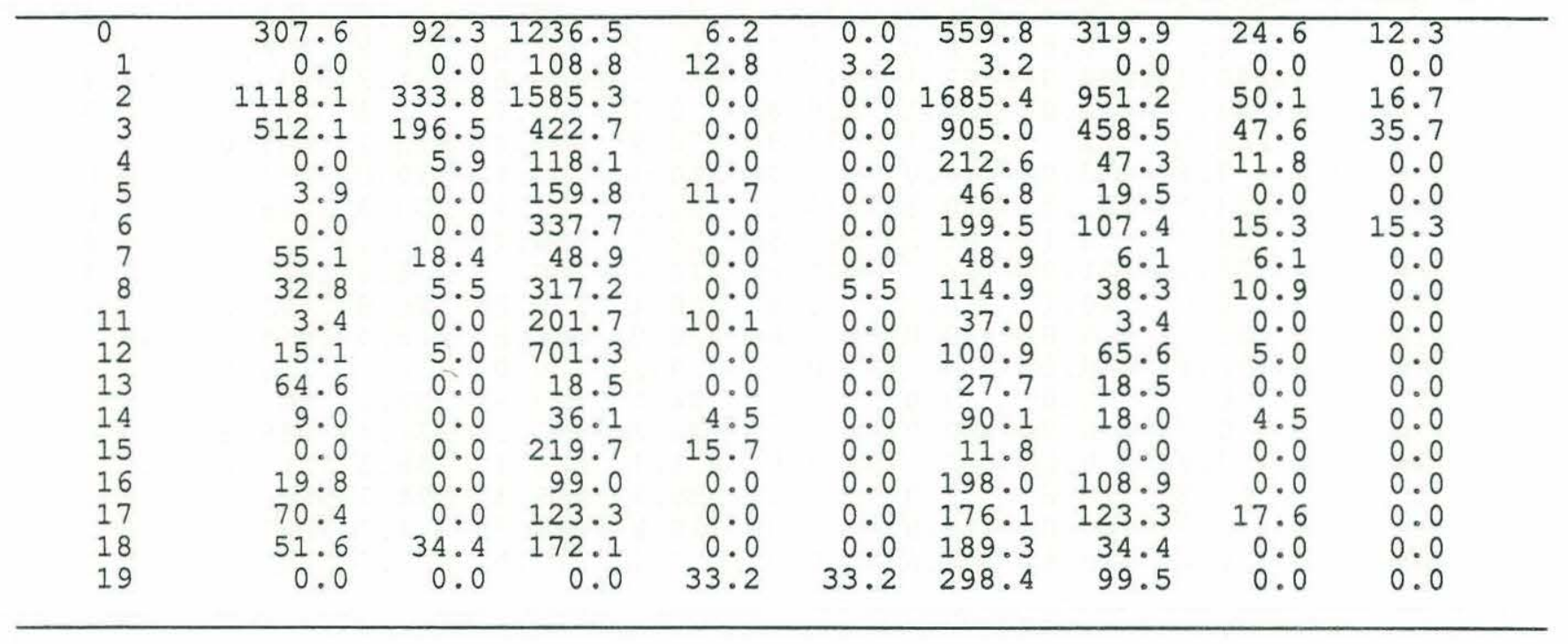

net\# \#ftur \#mtur \#fluc \#mluc \#cluc \#fbor \#fgra \#mpsm \#mpis

\begin{tabular}{rrrrrrrrrr}
\hline 0 & 0.0 & 0.0 & 6.2 & 6.2 & 73.8 & 0.0 & 0.0 & 0.0 & 0.0 \\
1 & 0.0 & 0.0 & 6.4 & 0.0 & 6.4 & 0.0 & 0.0 & 0.0 & 3.2 \\
2 & 133.5 & 0.0 & 0.0 & 0.0 & 0.0 & 0.0 & 0.0 & 0.0 & 0.0 \\
3 & 89.3 & 11.9 & 0.0 & 11.9 & 6.0 & 0.0 & 0.0 & 0.0 & 0.0 \\
4 & 70.9 & 35.4 & 0.0 & 0.0 & 0.0 & 0.0 & 0.0 & 0.0 & 0.0 \\
5 & 3.9 & 0.0 & 3.9 & 0.0 & 3.9 & 3.9 & 3.9 & 0.0 & 0.0 \\
6 & 199.5 & 0.0 & 0.0 & 0.0 & 0.0 & 0.0 & 0.0 & 0.0 & 0.0 \\
7 & 36.7 & 30.6 & 0.0 & 0.0 & 0.0 & 6.1 & 0.0 & 0.0 & 0.0 \\
8 & 16.4 & 5.5 & 0.0 & 0.0 & 10.9 & 0.0 & 0.0 & 0.0 & 0.0 \\
11 & 3.4 & 0.0 & 0.0 & 0.0 & 3.4 & 0.0 & 20.2 & 13.4 & 0.0 \\
12 & 10.1 & 0.0 & 0.0 & 0.0 & 0.0 & 0.0 & 0.0 & 0.0 & 0.0 \\
13 & 0.0 & 0.0 & 0.0 & 0.0 & 0.0 & 0.0 & 0.0 & 0.0 & 0.0 \\
14 & 9.0 & 0.0 & 0.0 & 0.0 & 0.0 & 0.0 & 0.0 & 0.0 & 0.0 \\
15 & 0.0 & 0.0 & 0.0 & 0.0 & 0.0 & 0.0 & 0.0 & 2.0 & 0.0 \\
16 & 0.0 & 0.0 & 0.0 & 0.0 & 0.0 & 0.0 & 0.0 & 0.0 & 0.0 \\
17 & 0.0 & 0.0 & 0.0 & 0.0 & 0.0 & 0.0 & 0.0 & 0.0 & 0.0 \\
18 & 0.0 & 0.0 & 0.0 & 0.0 & 0.0 & 0.0 & 0.0 & 0.0 & 0.0 \\
19 & 0.0 & 0.0 & 0.0 & 0.0 & 497.3 & 0.0 & 0.0 & 0.0 & 0.0 \\
& & & & & & & & & \\
\hline
\end{tabular}


mocness-1-cop217.std WCR-82H MOCNESS tow copepods

$4 b-4$

\begin{tabular}{|c|c|c|c|c|c|c|c|c|c|}
\hline net\# & \#cpsm & \#mabd & \#mrob & \#cplg & \#ftyp & \#mtyp & \#ffur & \#mfur & \#ccen \\
\hline $\begin{array}{r}0 \\
1 \\
2 \\
3 \\
4 \\
5 \\
6 \\
7 \\
8 \\
11 \\
12 \\
13 \\
14 \\
15 \\
16 \\
17 \\
18 \\
19\end{array}$ & $\begin{array}{r}0.0 \\
0.0 \\
0.0 \\
0.0 \\
0.0 \\
0.0 \\
0.0 \\
0.0 \\
0.0 \\
23.5 \\
0.0 \\
0.0 \\
4.5 \\
0.0 \\
0.0 \\
0.0 \\
0.0 \\
16.6\end{array}$ & $\begin{array}{l}0.0 \\
0.0 \\
0.0 \\
0.0 \\
0.0 \\
0.0 \\
0.0 \\
0.0 \\
5.5 \\
0.0 \\
0.0 \\
0.0 \\
0.0 \\
0.0 \\
0.0 \\
0.0 \\
0.0 \\
0.0\end{array}$ & $\begin{array}{l}6.2 \\
0.0 \\
0.0 \\
0.0 \\
0.0 \\
0.0 \\
0.0 \\
0.0 \\
0.0 \\
0.0 \\
0.0 \\
0.0 \\
0.0 \\
0.0 \\
0.0 \\
0.0 \\
0.0 \\
0.0\end{array}$ & $\begin{array}{r}12.3 \\
9.6 \\
0.0 \\
0.0 \\
0.0 \\
11.7 \\
0.0 \\
6.1 \\
10.9 \\
3.4 \\
0.0 \\
0.0 \\
0.0 \\
9.8 \\
0.0 \\
0.0 \\
103.3 \\
331.5\end{array}$ & $\begin{array}{r}0.0 \\
0.0 \\
0.0 \\
29.8 \\
35.4 \\
11.7 \\
30.7 \\
452.8 \\
333.7 \\
6.7 \\
111.0 \\
1310.6 \\
473.2 \\
7.8 \\
118.8 \\
7184.7 \\
3734.9 \\
5983.6\end{array}$ & $\begin{array}{r}6.2 \\
3.2 \\
0.0 \\
6.0 \\
5.9 \\
11.7 \\
0.0 \\
79.5 \\
169.6 \\
3.4 \\
55.5 \\
627.6 \\
234.3 \\
3.9 \\
158.4 \\
4772.2 \\
2340.8 \\
4740.5\end{array}$ & $\begin{array}{r}12.3 \\
0.0 \\
0.0 \\
29.8 \\
11.8 \\
0.0 \\
15.3 \\
0.0 \\
5.5 \\
0.0 \\
0.0 \\
0.0 \\
0.0 \\
0.0 \\
0.0 \\
0.0 \\
0.0 \\
16.6\end{array}$ & $\begin{array}{r}12.3 \\
3.2 \\
0.0 \\
11.9 \\
23.6 \\
0.0 \\
0.0 \\
6.1 \\
5.5 \\
0.0 \\
0.0 \\
0.0 \\
0.0 \\
0.0 \\
0.0 \\
0.0 \\
0.0 \\
33.2\end{array}$ & $\begin{array}{r}18.5 \\
3.2 \\
0.0 \\
23.8 \\
-999.0 \\
7.8 \\
76.7 \\
6.1 \\
125.8 \\
3.4 \\
100.9 \\
535.3 \\
599.4 \\
2.0 \\
277.2 \\
5758.3 \\
4389.0 \\
8403.6\end{array}$ \\
\hline
\end{tabular}

net\# \#fvio \#mvio \#cvio \#luci \#fhal \#chal \#farm \#marm \#fbip

\begin{tabular}{rrrrrrrrrr}
\hline 0 & 6.2 & 12.3 & 0.0 & 221.5 & 0.0 & 6.2 & 0.0 & 0.0 & 0.0 \\
1 & 9.6 & 0.0 & 0.0 & 765.0 & 60.8 & 83.2 & 0.0 & 0.0 & 0.0 \\
2 & 0.0 & 0.0 & 16.7 & 66.8 & 0.0 & 0.0 & 0.0 & 0.0 & 0.0 \\
3 & 41.7 & 29.8 & 35.7 & 6.0 & 0.0 & 0.0 & 0.0 & 0.0 & 0.0 \\
4 & 5.9 & 5.9 & 11.8 & 124.0 & 0.0 & 11.8 & 0.0 & 0.0 & 0.0 \\
5 & 11.7 & 0.0 & 0.0 & 518.3 & 42.9 & 54.6 & 0.0 & 0.0 & 0.0 \\
6 & 0.0 & 0.0 & 0.0 & 291.6 & 0.0 & 0.0 & 0.0 & 0.0 & 0.0 \\
7 & 128.5 & 61.2 & 61.2 & 0.0 & 0.0 & 0.0 & 0.0 & 0.0 & 0.0 \\
8 & 103.9 & 27.3 & 71.1 & 125.8 & 21.9 & 10.9 & 0.0 & 0.0 & 0.0 \\
11 & 10.1 & 0.0 & 0.0 & 396.6 & 20.2 & 26.9 & 0.0 & 0.0 & 0.0 \\
12 & 35.3 & 30.3 & 65.6 & 55.5 & 0.0 & 0.0 & 5.0 & 0.0 & 0.0 \\
13 & 249.2 & 286.1 & 92.3 & 0.0 & 0.0 & 0.0 & 36.9 & 18.5 & 0.0 \\
14 & 90.1 & 45.1 & 153.2 & 261.4 & 18.0 & 13.5 & 4.5 & 4.5 & 0.0 \\
15 & 9.8 & 7.8 & 0.0 & 757.3 & 104.0 & 15.7 & 0.0 & 0.0 & 0.0 \\
16 & 39.6 & 79.2 & 39.6 & 59.4 & 0.0 & 0.0 & 0.0 & 9.9 & 0.0 \\
17 & 457.8 & 334.6 & 105.7 & 35.2 & 0.0 & 0.0 & 70.4 & 17.6 & 17.6 \\
18 & 344.2 & 154.9 & 137.7 & 189.3 & 34.4 & 51.6 & 34.4 & 34.4 & 0.0 \\
19 & 961.4 & 795.6 & 679.6 & 1176.8 & 99.5 & 248.6 & 49.7 & 66.3 & 0.0 \\
\hline
\end{tabular}


mocness-1-cop217.std WCR-82H MOCNESS tow copepods

$4 b-5$

net\# \#fpak \#mpak \#fpbi \#mpbi \#fsim \#msim \#ccan \#fpon \#mpon

\begin{tabular}{rrrrrrrrrr}
\hline 0 & 123.0 & 147.6 & 6.2 & 0.0 & 18.5 & 0.0 & 879.7 & 6.2 & 12.3 \\
1 & 3.2 & 3.2 & 3.2 & 0.0 & 0.0 & 3.2 & 12.8 & 0.0 & 0.0 \\
2 & 66.8 & 33.4 & 0.0 & 0.0 & 0.0 & 50.1 & 100.1 & 0.0 & 16.7 \\
3 & 23.8 & 29.8 & 6.0 & 0.0 & 65.5 & 35.7 & 53.6 & 11.9 & 11.9 \\
4 & 11.8 & 23.6 & 0.0 & 0.0 & 0.0 & 0.0 & 70.9 & 17.7 & 0.0 \\
5 & 3.9 & 0.0 & 0.0 & 0.0 & 0.0 & 0.0 & 11.7 & 0.0 & 0.0 \\
6 & 0.0 & 0.0 & 0.0 & 0.0 & 0.0 & 0.0 & 15.3 & 0.0 & 0.0 \\
7 & 85.7 & 110.1 & 0.0 & 12.2 & 0.0 & 0.0 & 214.1 & 18.4 & 0.0 \\
8 & 38.3 & 43.8 & 0.0 & 0.0 & 10.9 & 5.5 & 38.3 & 10.9 & 0.0 \\
11 & 3.4 & 0.0 & 3.4 & 3.4 & 0.0 & 0.0 & 23.5 & 0.0 & 0.0 \\
12 & 0.0 & 0.0 & 0.0 & 0.0 & 0.0 & 0.0 & 15.1 & 0.0 & 0.0 \\
13 & 0.0 & 0.0 & 0.0 & 0.0 & 0.0 & 0.0 & 18.5 & 0.0 & 0.0 \\
14 & 0.0 & 0.0 & 0.0 & 0.0 & 0.0 & 0.0 & 18.0 & 0.0 & 0.0 \\
15 & 2.0 & 0.0 & 2.0 & 0.0 & 0.0 & 0.0 & 7.8 & 0.0 & 0.0 \\
16 & 0.0 & 0.0 & 0.0 & 0.0 & 0.0 & 0.0 & 19.8 & 0.0 & 0.0 \\
17 & 0.0 & 0.0 & 0.0 & 0.0 & 17.6 & 0.0 & 158.5 & 17.6 & 0.0 \\
18 & 0.0 & 0.0 & 0.0 & 0.0 & 17.2 & 0.0 & 137.7 & 0.0 & 0.0 \\
19 & 16.6 & 0.0 & 0.0 & 0.0 & 0.0 & 0.0 & 0.0 & 0.0 & 0.0 \\
\hline
\end{tabular}

\begin{tabular}{rrrrrrrl} 
net\# & \#acar & \#fmis & \#mmis & \#cmis & \#oith & \#cycl & \#tot 1 \\
\hline 0 & & & & & & & \\
\hline 1 & 03.1 & 221.5 & 67.7 & 1150.4 & 104.6 & 5069.2 & 813571.2 \\
2 & 1835.6 & 224.1 & 35.2 & 1510.9 & 112.0 & 332.9 & 4103.7 \\
3 & 53.6 & 190.5 & 100.1 & 1301.6 & 150.2 & 1768.9 & 824263.7 \\
4 & 17.7 & 218.5 & 41.3 & 5918.2 & 6.0 & 3489.1 & 8645.4 \\
5 & 0.0 & 93.5 & 39.0 & 1441.9 & 82.7 & 1364.4 & 5304.2 \\
6 & 460.5 & 168.8 & 107.4 & 445.1 & 153.5 & 552.8 & 3947.8 \\
7 & 61.2 & 110.1 & 36.7 & 336.5 & 24.5 & 1211.5 & 3630.9 \\
7 & 268.0 & 164.1 & 54.7 & 705.6 & 235.2 & 897.0 & 6820.7 \\
11 & 3.4 & 117.6 & 70.6 & 1129.4 & 356.3 & 275.6 & 4215.1 \\
12 & 999.0 & 121.1 & 30.3 & 529.8 & 1024.2 & 449.0 & 8511.6 \\
13 & 147.7 & 92.3 & 110.8 & 452.2 & 9.2 & 286.1 & 5639.1 \\
14 & 648.9 & 211.8 & 40.6 & 667.0 & 360.5 & 392.1 & 5876.5 \\
15 & 3.9 & 68.7 & 45.1 & 1396.9 & 92.2 & 213.9 & 4279.0 \\
16 & 49.5 & 217.8 & 9.9 & 722.6 & 821.6 & 138.6 & 7859.4 \\
17 & 228.9 & 968.5 & 633.9 & 4560.9 & 0.0 & 845.3 & 833669.4 \\
18 & 378.7 & 516.4 & 430.3 & 3476.8 & 223.8 & 774.5 & 825318.4 \\
19 & 1425.5 & 2403.4 & 928.2 & 8552.8 & 3232.2 & 1558.1 & 856289.2 \\
\hline
\end{tabular}


c217.int WCR-82H MOCNESS tow copepods

$4 c-1$

haul midd depi volf \#ffin \#mfin \#vfin \#cfin \#fhyp \#chyp

\begin{tabular}{rrrrrrrrrr}
\hline 1 & 61.5 & 123.0 & 650.2 & 0.0 & 0.0 & 6.8 & 1.5 & 0.0 & 2.3 \\
1 & 61.5 & 123.0 & 888.0 & 0.0 & 0.5 & 2.1 & 17.7 & 0.0 & 0.0 \\
2 & 63.0 & 126.0 & 677.2 & 0.0 & 0.0 & 1.5 & 26.0 & 0.7 & 0.0 \\
2 & 63.0 & 126.0 & 778.7 & 0.0 & 0.0 & 1.9 & 41.2 & 0.0 & 0.2 \\
3 & 60.0 & 120.0 & 731.3 & 0.0 & 0.0 & 3.3 & 78.1 & 0.0 & 0.0 \\
3 & 60.0 & 120.0 & 910.6 & 0.0 & 0.0 & 4.2 & 55.2 & 0.0 & 0.0 \\
4 & 60.0 & 120.0 & 887.6 & 0.0 & 0.0 & 4.9 & 31.9 & 0.0 & 0.0 \\
4 & 60.0 & 120.0 & 1368.1 & 0.9 & 0.9 & 4.2 & 59.4 & 0.0 & 0.0 \\
5 & 67.0 & 134.0 & 929.6 & 2.3 & 4.6 & 0.0 & 34.6 & 0.0 & 0.0 \\
5 & 67.0 & 134.0 & 965.3 & 0.0 & 0.0 & 2.2 & 2.2 & 0.0 & 0.0 \\
\hline
\end{tabular}

haul \#ften \#mten \#cten \#fngr \#mngr \#fnro \#mnro \#cneo \#fund

\begin{tabular}{rrrrrrrrrr}
\hline 1 & 2.3 & 0.0 & 0.8 & 0.0 & 0.0 & 0.0 & 0.0 & 4.5 & 25.0 \\
1 & 2.6 & 0.8 & 12.0 & 0.0 & 0.0 & 0.5 & 0.5 & 1.6 & 71.0 \\
2 & 2.2 & 0.0 & 3.7 & 0.0 & 0.7 & 0.0 & 0.0 & 0.0 & 28.3 \\
2 & 3.6 & 0.4 & 11.8 & 0.6 & 0.0 & 1.2 & 0.0 & 3.1 & 25.4 \\
3 & 2.6 & 0.7 & 7.2 & 0.0 & 0.0 & 0.7 & 0.0 & 2.0 & 4.6 \\
3 & 2.5 & 0.0 & 9.2 & 0.0 & 0.0 & 0.5 & 0.0 & 2.4 & 1.0 \\
4 & 2.2 & 0.0 & 6.5 & 0.0 & 0.0 & 0.0 & 0.0 & 1.1 & 0.0 \\
4 & 3.8 & 0.3 & 19.5 & 0.0 & 0.0 & 0.0 & 0.0 & 2.2 & 0.9 \\
5 & 9.2 & 0.0 & 13.8 & 0.0 & 0.0 & 0.0 & 0.0 & 2.3 & 0.0 \\
5 & 11.1 & 0.0 & 135.5 & 0.0 & 0.0 & 0.0 & 0.0 & 2.2 & 0.0 \\
\hline
\end{tabular}

haul \#mund \#vund \#cund \#ccal \#fmin \#mmin \#cmin \#felg \#celg

\begin{tabular}{rrrrrrrrrr}
\hline 1 & 21.9 & 27.2 & 4.5 & 3.0 & 21.9 & 21.2 & 174.8 & 0.0 & 0.0 \\
1 & 37.5 & 54.6 & 12.1 & 14.6 & 59.4 & 29.8 & 114.7 & 0.5 & 0.0 \\
2 & 10.4 & 23.1 & 8.9 & 8.9 & 36.5 & 14.1 & 87.8 & 0.0 & 0.0 \\
2 & 22.6 & 10.0 & 7.1 & 18.4 & 33.9 & 12.1 & 114.7 & 0.0 & 1.2 \\
3 & 2.6 & 6.6 & 3.3 & 21.0 & 28.9 & 11.2 & 125.4 & 1.3 & 2.0 \\
3 & 1.2 & 0.8 & 0.2 & 25.0 & 31.2 & 8.2 & 109.3 & 1.6 & 4.1 \\
4 & 0.0 & 0.0 & 0.0 & 4.3 & 23.3 & 7.6 & 68.1 & 0.0 & 1.6 \\
4 & 0.4 & 0.0 & 0.0 & 28.9 & 57.2 & 36.6 & 139.1 & 1.4 & 11.1 \\
5 & 0.0 & 0.0 & 0.0 & 41.5 & 138.4 & 62.3 & 435.9 & 0.0 & 9.2 \\
5 & 2.2 & 2.2 & 0.0 & 248.8 & 131.0 & 111.1 & 1012.8 & 0.0 & 2.2 \\
\hline
\end{tabular}


c217.int WCR-82H MOCNESS tow copepods

$4 c-2$

haul \#fcor \#mcor \#ccor \#fnas \#cnas \#fmey \#fpav \#farc \#fmar

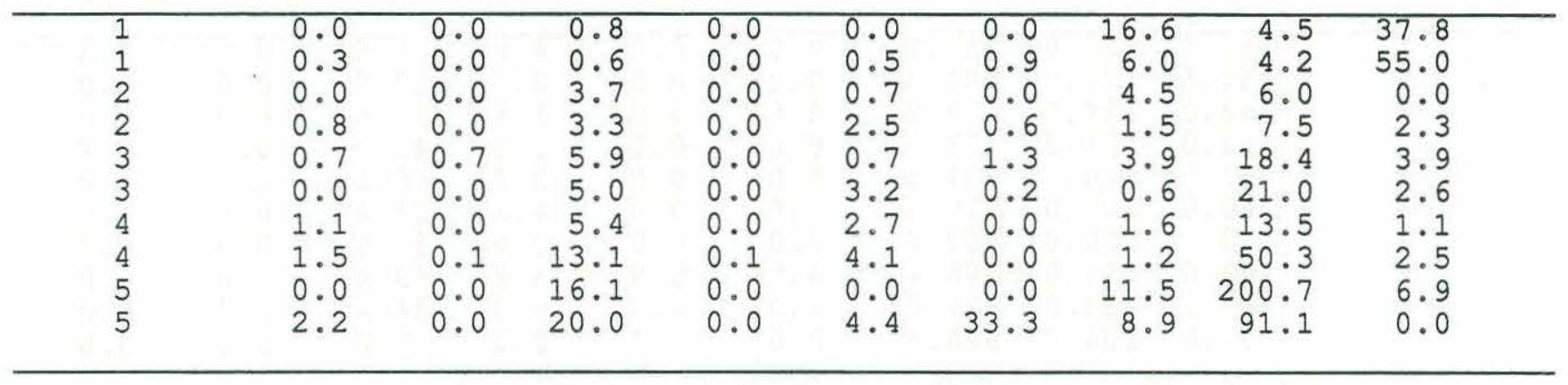

haul \#mmar \#ceuk \#fbra \#mbra \#fdan \#mdan \#ftst \#mtst \#ftur

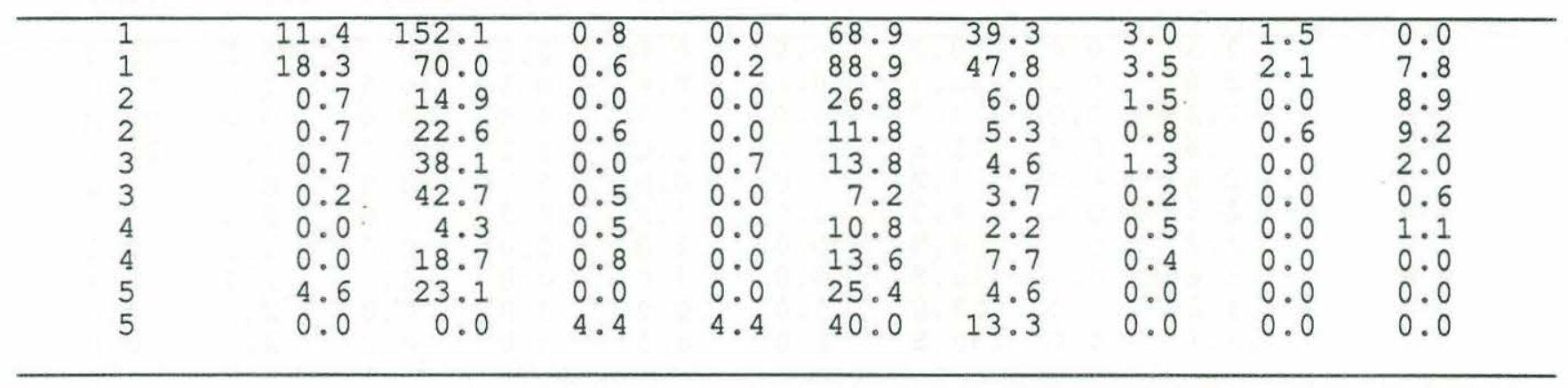

haul \#mtur \#fluc \#mluc \#cluc \#fbor \#fgra \#mpsm \#mpis \#cpsm

\begin{tabular}{llllllllllll}
\hline 1 & 0.0 & 0.8 & 0.8 & 9.1 & 0.0 & 0.0 & 0.0 & 0.0 & 0.0 \\
1 & 0.5 & 0.3 & 0.5 & 0.6 & 0.0 & 0.0 & 0.0 & 0.2 & 0.0 & 0 \\
2 & 4.5 & 0.0 & 0.0 & 0.0 & 0.0 & 0.0 & 0.0 & 0.0 & 0.0 & 0 \\
2 & 1.2 & 0.2 & 0.0 & 0.2 & 0.4 & 0.2 & 0.0 & 0.0 & 0.0 & 0 \\
3 & 0.7 & 0.0 & 0.0 & 1.3 & 0.0 & 0.0 & 0.0 & 0.0 & 0.0 \\
3 & 0.0 & 0.0 & 0.0 & 0.2 & 0.0 & 0.9 & 0.6 & 0.0 & 1.1 & 0.0 \\
4 & 0.0 & 0.0 & 0.0 & 0.0 & 0.0 & 0.0 & 0.0 & 0.0 & 0.5 & 0.0 \\
4 & 0.0 & 0.0 & 0.0 & 0.0 & 0.0 & 0.0 & 0.1 & 0.0 & 0.0 \\
5 & 0.0 & 0.0 & 0.0 & 0.0 & 0.0 & 0.0 & 0.0 & 0.0 & 0.0 & 0.0 \\
5 & 0.0 & 0.0 & 0.0 & 66.6 & 0.0 & 0.0 & 0.0 & 0.0 & 2.2 \\
\hline
\end{tabular}


c217.int WCR-82H MOCNESS tow copepods

$4 c-3$

haul \#mabd \#mrob \#cplg \#ftyp \#mtyp \#ffur \#mfur \#ccen \#fvio

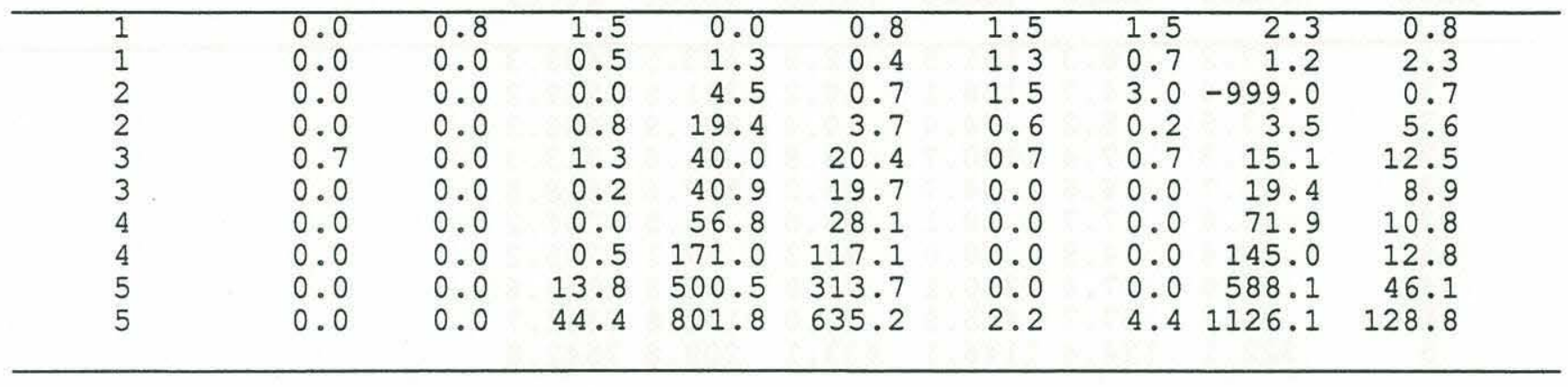

haul \#mvio \#cvio \#luci \#fhal \#chal \#farm \#marm \#fbip \#fpak

\begin{tabular}{rrrrrrrrrrr}
\hline 1 & 1.5 & 0.0 & 27.2 & 0.0 & 0.8 & 0.0 & 0.0 & 0.0 & 15.1 \\
1 & 1.3 & 2.1 & 40.5 & 3.0 & 4.2 & 0.0 & 0.0 & 0.0 & 3.1 & 0.0 \\
2 & 0.7 & 1.5 & 15.6 & 0.0 & 1.5 & 0.0 & 0.0 & 0.0 & 1.5 \\
2 & 2.4 & 2.4 & 36.5 & 2.1 & 2.7 & 0.0 & 0.0 & 0.0 & 3.5 \\
3 & 3.3 & 8.5 & 15.1 & 2.6 & 1.3 & 0.0 & 0.0 & 0.0 & 4.6 & 0 \\
3 & 9.1 & 5.6 & 20.9 & 0.9 & 1.2 & 1.2 & 0.5 & 0.0 & 0.2 & 0 \\
4 & 5.4 & 18.4 & 31.4 & 2.2 & 1.6 & 0.5 & 0.5 & 0.0 & 0.0 & 0.9 \\
4 & 11.7 & 4.2 & 42.9 & 5.4 & 0.8 & 1.6 & 0.9 & 0.4 & 0.1 \\
5 & 20.8 & 18.5 & 25.4 & 4.6 & 6.9 & 4.6 & 4.6 & 0.0 & 0.0 \\
5 & 106.6 & 91.1 & 157.7 & 13.3 & 33.3 & 6.7 & 8.9 & 0.0 & 2.2 \\
\hline
\end{tabular}

haul \#mpak \#fpbi \#mpbi \#fsim \#msim \#ccan \#fpon \#mpon \#acar

\begin{tabular}{|c|c|c|c|c|c|c|c|c|c|}
\hline $\begin{array}{l}1 \\
1 \\
2 \\
2 \\
3 \\
3 \\
4 \\
4 \\
5 \\
5\end{array}$ & $\begin{array}{r}18.2 \\
2.4 \\
3.0 \\
4.3 \\
5.3 \\
0.0 \\
0.0 \\
0.0 \\
0.0 \\
0.0\end{array}$ & $\begin{array}{l}0.8 \\
0.4 \\
0.0 \\
0.0 \\
0.0 \\
0.2 \\
0.0 \\
0.1 \\
0.0 \\
0.0\end{array}$ & $\begin{array}{l}0.0 \\
0.0 \\
0.0 \\
0.5 \\
0.0 \\
0.2 \\
0.0 \\
0.0 \\
0.0 \\
0.0\end{array}$ & $\begin{array}{l}2.3 \\
2.9 \\
0.0 \\
0.0 \\
1.3 \\
0.0 \\
0.0 \\
0.4 \\
2.3 \\
0.0\end{array}$ & $\begin{array}{l}0.0 \\
3.2 \\
0.0 \\
0.0 \\
0.7 \\
0.0 \\
0.0 \\
0.0 \\
0.0 \\
0.0\end{array}$ & $\begin{array}{r}108.2 \\
5.9 \\
8.9 \\
9.5 \\
4.6 \\
2.3 \\
2.2 \\
4.9 \\
18.5 \\
0.0\end{array}$ & $\begin{array}{l}0.8 \\
0.5 \\
2.2 \\
0.7 \\
1.3 \\
0.0 \\
0.0 \\
0.4 \\
0.0 \\
0.0\end{array}$ & $\begin{array}{l}1.5 \\
1.0 \\
0.0 \\
0.0 \\
0.0 \\
0.0 \\
0.0 \\
0.0 \\
0.0 \\
0.0\end{array}$ & $\begin{array}{r}5.3 \\
55.6 \\
2.2 \\
19.9 \\
32.2 \\
51.1 \\
77.9 \\
7.7 \\
50.7 \\
191.0\end{array}$ \\
\hline
\end{tabular}


c217.int WCR-82H MOCNESS tow copepods

$4 c-4$

\begin{tabular}{rrrrrrr} 
haul & \#fmis & \#mmis & \#cmis & \#oith & \#cycl & \#totl \\
\hline 1 & & & & & & \\
\hline 1 & 19.2 & 8.3 & 141.5 & 12.9 & 623.5 & 1669.3 \\
2 & 27.5 & 4.7 & 158.1 & 10.2 & 221.5 & 1289.2 \\
2 & 15.3 & 7.4 & 74.4 & 10.4 & 171.9 & 668.3 \\
3 & 19.7 & 6.6 & 84.7 & 8.9 & 81.6 & 713.3 \\
3 & 13.6 & 7.7 & 89.1 & 64.8 & 107.6 & 818.5 \\
4 & 25.4 & 4.9 & 80.0 & 43.3 & 47.1 & 746.2 \\
4 & 35.6 & 17.4 & 210.1 & 41.8 & 36.8 & 1350.6 \\
5 & 69.2 & 57.7 & 465.9 & 30.0 & 103.8 & 3392.7 \\
5 & 322.1 & 124.4 & 1146.1 & 433.1 & 208.8 & 7542.8 \\
\hline
\end{tabular}


c218.raw WCR-82H MOCNESS tow copepods

$4 d-1$

\begin{tabular}{|c|c|c|c|c|c|c|c|c|c|}
\hline net\# & midd & depi & volf & aliq & ffin & vfin & cfin & fhyp & chyp \\
\hline 18 & 13 & 25 & 184 & 4 & 0 & 0 & 0 & 0 & 0 \\
\hline 17 & 38 & 25 & 151 & 8 & 0 & 1 & 0 & 0 & 0 \\
\hline 16 & 63 & 25 & 147 & 8 & 0 & 0 & 0 & 0 & 0 \\
\hline 15 & 88 & 25 & 140 & 2 & 0 & 0 & 0 & 0 & 0 \\
\hline 14 & 113 & 25 & 128 & 2 & 0 & 0 & 0 & 0 & 0 \\
\hline 13 & 138 & 25 & 125 & 2 & 0 & 1 & 0 & 0 & 0 \\
\hline 12 & 163 & 25 & 116 & 1 & 0 & 1 & 1 & 0 & 0 \\
\hline 11 & 188 & 25 & 164 & 1 & 0 & 0 & 0 & 0 & 0 \\
\hline 8 & 250 & 100 & 752 & 4 & 0 & 1 & 6 & 0 & 0 \\
\hline 7 & 350 & 100 & 662 & 2 & 0 & 0 & 1 & 0 & 0 \\
\hline 6 & 450 & 100 & 489 & 1 & 1 & 5 & 6 & 0 & 0 \\
\hline 5 & 550 & 100 & 514 & 4 & 0 & 6 & 5 & 0 & 0 \\
\hline 4 & 650 & 100 & 858 & 4 & 3 & 188 & 72 & 0 & 3 \\
\hline 3 & 750 & 100 & 532 & 2 & 7 & 452 & 169 & 0 & 4 \\
\hline 2 & 850 & 100 & 424 & 2 & 15 & 408 & 188 & 5 & 5 \\
\hline 1 & 950 & 100 & 340 & 1 & 9 & 346 & 163 & 5 & 10 \\
\hline
\end{tabular}

\begin{tabular}{|c|c|c|c|c|c|c|c|c|c|}
\hline net\# & ften & mten & cten & fngr & mngr & fnro & cneo & fund & mund \\
\hline 18 & 1 & 0 & 0 & 12 & 0 & 1 & 21 & 23 & 20 \\
\hline 17 & 0 & 0 & 1 & 0 & 0 & 0 & 6 & 4 & 3 \\
\hline 16 & 19 & 0 & 33 & 0 & 0 & 0 & 2 & 3 & 2 \\
\hline 15 & 26 & 13 & 74 & 0 & 0 & 1 & 15 & 0 & 0 \\
\hline 14 & 3 & 2 & 3 & 0 & 0 & 1 & 4 & 0 & 0 \\
\hline 13 & 0 & 3 & 2 & 4 & 0 & 1 & 4 & 0 & 0 \\
\hline 12 & 0 & 0 & 1 & 2 & 0 & 0 & 2 & 0 & 0 \\
\hline 11 & 1 & 3 & 1 & 0 & 0 & 0 & 2 & 0 & 0 \\
\hline 8 & 0 & 0 & 0 & 2 & 0 & 0 & 0 & 0 & 0 \\
\hline 7 & 0 & 0 & 0 & 1 & 0 & 0 & 8 & 0 & 0 \\
\hline 6 & 0 & 0 & 0 & 0 & 18 & 0 & 10 & 0 & 0 \\
\hline 5 & 0 & 0 & 0 & 0 & 7 & 0 & 3 & 0 & 0 \\
\hline 4 & 0 & 0 & 0 & 0 & 3 & 0 & 0 & 0 & 0 \\
\hline 3 & 0 & 0 & 0 & 0 & 1 & 0 & 0 & 0 & 0 \\
\hline 2 & 0 & 0 & 0 & 0 & 0 & 0 & 0 & 0 & 0 \\
\hline 1 & 0 & 0 & 0 & 0 & 0 & 0 & 0 & 0 & 0 \\
\hline
\end{tabular}


c218.raw WCR-82H MOCNESS tow copepods

$4 d-2$

\begin{tabular}{|c|c|c|c|c|c|c|c|c|c|}
\hline net\# & cund & ccal & fmin & mmin & cmin & felg & celg & fcor & mcor \\
\hline 18 & 260 & 26 & 55 & 25 & 75 & 0 & $\overline{0}$ & 0 & 0 \\
\hline 17 & 87 & 3 & 12 & 5 & 47 & 0 & 0 & 0 & 0 \\
\hline 16 & 27 & 10 & 10 & 6 & 21 & 0 & 0 & 0 & 0 \\
\hline 15 & 1 & 28 & 0 & 1 & 0 & 0 & 0 & 0 & 0 \\
\hline 14 & 0 & 13 & 3 & 1 & 3 & 1 & 1 & 1 & 0 \\
\hline 13 & 0 & 7 & 1 & 0 & 0 & 0 & 0 & 1 & 0 \\
\hline 12 & 0 & 7 & 2 & 0 & 1 & 0 & 0 & 0 & 0 \\
\hline 11 & 0 & 9 & 0 & 0 & 3 & 0 & 0 & 0 & 1 \\
\hline 8 & 0 & 12 & 0 & 0 & 1 & 0 & 0 & 2 & 0 \\
\hline 7 & 1 & 2 & 0 & 0 & 0 & 0 & 0 & 2 & 0 \\
\hline 6 & 0 & 1 & 0 & 0 & 0 & 0 & 2 & 6 & 0 \\
\hline 5 & 0 & 0 & 0 & 0 & 0 & 0 & 8 & 13 & 1 \\
\hline 4 & 0 & 0 & 0 & 0 & 1 & 7 & 8 & 39 & 8 \\
\hline 3 & 0 & 0 & 0 & 0 & 0 & 48 & 10 & 26 & 4 \\
\hline 2 & 0 & 2 & 0 & 0 & 3 & 9 & 3 & 2 & 0 \\
\hline 1 & 0 & 1 & 2 & 0 & 1 & 4 & 6 & 4 & 0 \\
\hline
\end{tabular}

\begin{tabular}{|c|c|c|c|c|c|c|c|c|c|}
\hline net\# & ccor & fnas & cnas & fmey & facr & fprv & farc & ffur & fege \\
\hline 18 & 3 & 0 & 0 & 0 & 72 & 11 & 61 & 14 & 0 \\
\hline 17 & 1 & 0 & 0 & 1 & 14 & 5 & 28 & 11 & 0 \\
\hline 16 & 0 & 0 & 0 & 0 & 7 & 2 & 29 & 0 & 0 \\
\hline 15 & 0 & 0 & 0 & 0 & 0 & 1 & 47 & 3 & 3 \\
\hline 14 & 1 & 0 & 0 & 0 & 0 & 1 & 16 & 0 & 4 \\
\hline 13 & 0 & 0 & 0 & 0 & 0 & 0 & 8 & 0 & 0 \\
\hline 12 & 0 & 0 & 0 & 0 & 0 & 0 & 4 & 1 & 1 \\
\hline 11 & 2 & 0 & 0 & 0 & 0 & 1 & 4 & 0 & 1 \\
\hline 8 & 8 & 0 & 0 & 0 & 0 & 0 & 5 & 0 & 0 \\
\hline 7 & 28 & 0 & 0 & 0 & 0 & 0 & 0 & 0 & 0 \\
\hline 6 & 56 & 0 & 0 & 0 & 0 & 0 & 0 & 0 & 0 \\
\hline 5 & 53 & 0 & 0 & 0 & 0 & 0 & 0 & 0 & 0 \\
\hline 4 & 49 & 0 & 0 & 0 & 0 & 0 & 2 & 0 & 0 \\
\hline 3 & 15 & 0 & 2 & 0 & 0 & 0 & 0 & 0 & 0 \\
\hline 2 & 4 & 0 & 4 & 0 & 0 & 0 & 0 & 0 & 1 \\
\hline 1 & 5 & 2 & 2 & 0 & 0 & 0 & 0 & 0 & 1 \\
\hline
\end{tabular}


c218.raw WCR-82H MOCNESS tow copepods

$4 d-3$

\begin{tabular}{|c|c|c|c|c|c|c|c|c|c|}
\hline net\# & feme & meme & fupl & mupl & fumj & fchi & fmed & mmed & fmar \\
\hline 18 & 2 & 0 & 13 & 8 & 0 & 0 & 4 & 0 & 4 \\
\hline 17 & 1 & 1 & 1 & 0 & 0 & 0 & 5 & 0 & 3 \\
\hline 16 & 1 & 0 & 0 & 0 & 0 & 0 & 5 & 0 & 1 \\
\hline 15 & 4 & 1 & 0 & 2 & 1 & 0 & 14 & 11 & 0 \\
\hline 14 & 1 & 1 & 5 & 0 & 0 & 1 & 2 & 1 & 0 \\
\hline 13 & 4 & 2 & 14 & 2 & 1 & 3 & 4 & 3 & 0 \\
\hline 12 & 1 & 1 & 4 & 0 & 1 & 3 & 1 & 1 & 0 \\
\hline 11 & 0 & .0 & 2 & 1 & 0 & 2 & 1 & 1 & 0 \\
\hline 8 & 0 & 0 & 2 & 0 & 0 & 1 & 1 & 0 & 0 \\
\hline 7 & 0 & 0 & 1 & 1 & 0 & 3 & 0 & 0 & 0 \\
\hline 6 & 0 & 0 & 1 & 0 & 0 & 2 & 1 & 3 & 0 \\
\hline 5 & 0 & 0 & 0 & 0 & 0 & 0 & 2 & 1 & 0 \\
\hline 4 & 8 & 0 & 4 & 5 & 0 & 4 & 7 & 0 & 0 \\
\hline 3 & 3 & 0 & 10 & 1 & 0 & 0 & 0 & 0 & 0 \\
\hline 2 & 1 & 0 & 6 & 2 & 1 & 1 & 0 & 0 & 2 \\
\hline 1 & 2 & 0 & 6 & 7 & 0 & 0 & 0 & 0 & 0 \\
\hline
\end{tabular}

\begin{tabular}{|c|c|c|c|c|c|c|c|c|c|}
\hline net\# & mmar & fsco & msco & fbra & mbra & fdan & mdan & fluc & mluc \\
\hline 18 & 0 & 0 & 0 & 2 & 0 & 24 & 29 & 0 & 0 \\
\hline 17 & 3 & 0 & 0 & 1 & 0 & 3 & 1 & 0 & 0 \\
\hline 16 & 1 & 0 & 0 & 4 & 0 & 0 & 0 & 0 & 0 \\
\hline 15 & 0 & 0 & 1 & 6 & 2 & 4 & 0 & 0 & 0 \\
\hline 14 & 0 & 2 & 1 & 1 & 0 & 0 & 0 & 0 & 0 \\
\hline 13 & 0 & 2 & 1 & 3 & 0 & 1 & 0 & 0 & 0 \\
\hline 12 & 0 & 1 & 1 & 1 & 2 & 1 & 0 & 0 & 0 \\
\hline 11 & 0 & 1 & 1 & 0 & 0 & 0 & 0 & 0 & 0 \\
\hline 8 & 0 & 2 & 0 & 0 & 0 & 0 & 0 & 0 & 0 \\
\hline 7 & 0 & 1 & 0 & 1 & 0 & 0 & 0 & 0 & 0 \\
\hline 6 & 0 & 2 & 0 & 1 & 0 & 0 & 1 & 0 & 0 \\
\hline 5 & 0 & 0 & 0 & 1 & 0 & 0 & 0 & 0 & 0 \\
\hline 4 & 0 & 0 & 0 & 0 & 0 & 0 & 0 & 0 & 0 \\
\hline 3 & 0 & 1 & 0 & 0 & 0 & 1 & 0 & 0 & 1 \\
\hline 2 & 0 & 0 & 0 & 0 & 0 & 4 & 0 & 0 & 1 \\
\hline 1 & 0 & 0 & 0 & 0 & 0 & 0 & 0 & 4 & 22 \\
\hline
\end{tabular}


c218.raw WCR-82H MOCNESS tow copepods

$4 d^{-} 4$

\begin{tabular}{|c|c|c|c|c|c|c|c|c|c|}
\hline net\# & fven & mven & fbrv & mbrv & cmet & fgra & mgra & fpis & mpis \\
\hline 18 & 0 & 0 & 0 & 0 & 0 & 18 & 2 & 26 & 0 \\
\hline 17 & 0 & 0 & 0 & 0 & 0 & 136 & 23 & 22 & 16 \\
\hline 16 & 0 & 0 & 0 & 0 & 0 & 22 & 25 & 3 & 7 \\
\hline 15 & 0 & 0 & 0 & 0 & 0 & 4 & 15 & 4 & 0 \\
\hline 14 & 0 & 0 & 0 & 0 & 1 & 3 & 2 & 0 & 0 \\
\hline 13 & 0 & 0 & 1 & 1 & 0 & 4 & 1 & 1 & 0 \\
\hline 12 & 0 & 0 & 0 & 0 & 0 & 1 & 1 & 0 & 0 \\
\hline 11 & 0 & 0 & 0 & 0 & 0 & 2 & 2 & 0 & 0 \\
\hline 8 & 0 & 0 & 0 & 0 & 0 & 1 & 0 & 0 & 0 \\
\hline 7 & 0 & 0 & 0 & 0 & 0 & 0 & 0 & 0 & 0 \\
\hline 6 & 0 & 1 & 0 & 0 & 0 & 4 & 12 & 0 & 0 \\
\hline 5 & 2 & 3 & 1 & 0 & 19 & 3 & 10 & 4 & 6 \\
\hline 4 & 14 & 44 & 36 & 9 & 81 & 0 & 1 & 1 & 1 \\
\hline 3 & 67 & 18 & 24 & 38 & 24 & 0 & 1 & 0 & 0 \\
\hline 2 & 14 & 0 & 5 & 4 & 26 & 0 & 0 & 0 & 0 \\
\hline 1 & 0 & 0 & 9 & 4 & 134 & 0 & 0 & 0 & 0 \\
\hline
\end{tabular}

\begin{tabular}{|c|c|c|c|c|c|c|c|c|c|}
\hline net\# & cpsm & fabd & mabd & fxip & mxip & cplg & fvio & mvio & ftyp \\
\hline 18 & 40 & 22 & 1 & 0 & 0 & 30 & 23 & 18 & 5 \\
\hline 17 & 77 & 6 & 2 & 0 & 0 & 86 & 7 & 2 & 15 \\
\hline 16 & 40 & 8 & 9 & 0 & 0 & 45 & 1 & 1 & 4 \\
\hline 15 & 7 & 6 & 12 & 13 & 9 & 60 & 0 & 0 & 2 \\
\hline 14 & 4 & 1 & 0 & 17 & 10 & 17 & 0 & 1 & 0 \\
\hline 13 & 2 & 2 & 2 & 63 & 10 & 1 & 0 & 0 & 2 \\
\hline 12 & 2 & 0 & 2 & 11 & 3 & 2 & 0 & 1 & 0 \\
\hline 11 & 2 & 0 & 0 & 2 & 3 & 1 & 1 & 1 & 9 \\
\hline 8 & 3 & 0 & 0 & 0 & 0 & 0 & 0 & 1 & 2 \\
\hline 7 & 27 & 0 & 0 & 2 & 0 & 74 & 0 & 0 & 0 \\
\hline 6 & 118 & 0 & 0 & 0 & 0 & 69 & 0 & 0 & 2 \\
\hline 5 & 22 & 0 & 3 & 0 & 0 & 32 & 0 & 0 & 0 \\
\hline 4 & 2 & 2 & 7 & 1 & 10 & 32 & 0 & 0 & 0 \\
\hline 3 & 0 & 9 & 2 & 7 & 9 & 28 & 0 & 0 & 0 \\
\hline 2 & 0 & 0 & 3 & 0 & 1 & 4 & 0 & 0 & 2 \\
\hline 1 & 0 & 0 & 0 & 0 & 1 & 0 & 0 & 0 & 0 \\
\hline
\end{tabular}




\section{c218.raw WCR-82H MOCNESS tow copepods}

$4 d-5$

\begin{tabular}{|c|c|c|c|c|c|c|c|c|c|}
\hline net\# & mtyp & luci & fpap & mpap & fspi & mspi & fvip & mvip & fhal \\
\hline 18 & 8 & 244 & 3 & 4 & 0 & 0 & 0 & 0 & 1 \\
\hline 17 & 9 & 114 & 4 & 5 & 0 & 0 & 0 & 0 & 0 \\
\hline 16 & 3 & 64 & 9 & 6 & 0 & 0 & 0 & 0 & 18 \\
\hline 15 & 1 & 170 & 11 & 7 & 1 & 0 & 0 & 0 & 40 \\
\hline 14 & 5 & . 95 & 6 & 8 & 0 & 4 & 0 & 0 & 9 \\
\hline 13 & 3 & 63 & 24 & 18 & 4 & 5 & 0 & 0 & 31 \\
\hline 12 & 0 & 31 & 18 & 9 & 2 & 3 & 0 & 0 & 42 \\
\hline 11 & 4 & 95 & 15 & 3 & 2 & 0 & 0 & 0 & 56 \\
\hline 8 & 3 & 116 & 9 & 4 & 3 & 0 & 3 & 1 & 65 \\
\hline 7 & 1 & 98 & 12 & 2 & 2 & 1 & 4 & 6 & 242 \\
\hline 6 & 0 & 72 & 29 & 32 & 1 & 0 & 10 & 6 & 163 \\
\hline 5 & 0 & 6 & 6 & 0 & 1 & 2 & 2 & 3 & 18 \\
\hline 4 & 0 & 14 & 6 & 2 & 5 & 1 & 3 & 5 & 3 \\
\hline 3 & 0 & 23 & 0 & 0 & 1 & 0 & 5 & 2 & 0 \\
\hline 2 & 3 & 12 & 0 & 0 & 0 & 0 & 1 & 0 & 0 \\
\hline 1 & 5 & 5 & 0 & 0 & 0 & 0 & 0 & 0 & 2 \\
\hline
\end{tabular}

\begin{tabular}{|c|c|c|c|c|c|c|c|c|c|}
\hline net\# & mhal & chal & fhel & mhel & fket & mket & fclo & mclo & fpak \\
\hline 18 & 0 & 1 & 0 & 1 & 0 & 0 & 0 & 0 & 1 \\
\hline 17 & 0 & 1 & 0 & 0 & 0 & 0 & 0 & 0 & 0 \\
\hline 16 & 0 & 0 & 0 & 0 & 0 & 0 & 1 & 0 & 1 \\
\hline 15 & 3 & 33 & 1 & 0 & 0 & 0 & 4 & 1 & 0 \\
\hline 14 & 1 & 89 & 0 & 5 & 0 & 0 & 2 & 4 & 0 \\
\hline 13 & 0 & 301 & 6 & 7 & 0 & 0 & 2 & 3 & 0 \\
\hline 12 & 1 & 244 & 4 & 3 & 1 & 1 & 0 & 0 & 0 \\
\hline 11 & 0 & 369 & 6 & 7 & 0 & 0 & 0 & 0 & 0 \\
\hline 8 & 0 & 302 & 6 & 8 & 0 & 1 & 0 & 0 & 0 \\
\hline 7 & 5 & 103 & 2 & 0 & 0 & 1 & 0 & 0 & 0 \\
\hline 6 & 1 & 86 & 5 & 2 & 0 & 0 & 3 & 0 & 0 \\
\hline 5 & 0 & 10 & 3 & 0 & 0 & 0 & 0 & 0 & 0 \\
\hline 4 & 0 & 0 & 19 & 19 & 0 & 0 & 0 & 0 & 0 \\
\hline 3 & 0 & 0 & 11 & 13 & 0 & 0 & 0 & 0 & 0 \\
\hline 2 & 0 & 1 & 0 & 1 & 0 & 0 & 0 & 0 & 0 \\
\hline 1 & 0 & 0 & 0 & 1 & 0 & 0 & 0 & 0 & 0 \\
\hline
\end{tabular}


c218.raw WCR-82H MOCNESS tow copepods

$4 d-6$

\begin{tabular}{|c|c|c|c|c|c|c|c|c|c|}
\hline net\# & mpak & fpbi & mpbi & mpsi & acar & fmis & mmis & cmis & cycl \\
\hline 18 & 0 & 7 & 6 & 0 & 103 & 43 & 12 & 479 & 523 \\
\hline 17 & 1 & 2 & 3 & 0 & 24 & 19 & 9 & 301 & 192 \\
\hline 16 & 1 & 10 & 10 & 0 & 15 & 31 & 2 & 210 & 156 \\
\hline 15 & 0 & 3 & 5 & 0 & 7 & 71 & 12 & 316 & 320 \\
\hline 14 & 0 & 0 & 0 & 0 & 6 & 34 & 14 & 231 & 256 \\
\hline 13 & 0 & 0 & 0 & 1 & 1 & 27 & 6 & 213 & 106 \\
\hline 12 & 0 & 0 & 0 & 0 & 4 & 37 & 10 & 180 & 71 \\
\hline 11 & 0 & 0 & 0 & 0 & 3 & 26 & 9 & 212 & 123 \\
\hline 8 & 0 & 0 & 0 & 0 & 1 & 41 & 17 & 236 & 105 \\
\hline 7 & 0 & 0 & 0 & 0 & 1 & 85 & 24 & 282 & 111 \\
\hline 6 & 0 & 0 & 0 & 0 & 1 & 149 & 47 & 629 & 57 \\
\hline 5 & 0 & 0 & 0 & 0 & 0 & 105 & 28 & 335 & 82 \\
\hline 4 & 0 & 0 & 0 & 0 & 0 & 199 & 87 & 453 & 57 \\
\hline 3 & 0 & 0 & 0 & 0 & 0 & 120 & 29 & 334 & 18 \\
\hline 2 & 0 & 0 & 0 & 0 & 0 & 88 & 18 & 277 & 30 \\
\hline 1 & 0 & 0 & 0 & 0 & 2 & 103 & 18 & 362 & 34 \\
\hline
\end{tabular}

\begin{tabular}{rrr} 
net\# & harp & tot 1 \\
\hline 18 & 22 & 2408 \\
17 & 6 & 1330 \\
16 & 5 & 890 \\
15 & 1 & 1399 \\
14 & 0 & 898 \\
13 & 1 & 983 \\
12 & 0 & 725 \\
11 & 1 & 991 \\
8 & 2 & 973 \\
7 & 2 & 1137 \\
6 & 0 & 1617 \\
5 & 2 & 808 \\
4 & 6 & 1531 \\
3 & 1 & 1545 \\
2 & 0 & 1157 \\
1 & 4 & 1287 \\
\hline
\end{tabular}


c218.std WCR-82H MOCNESS tow copepods

4e 1

\begin{tabular}{rrrrrrrrrr} 
net\# & midd & depi & volf & aliq & \#ffin & \#vfin & \#cfin & \#fhyp \#chyp & \# \\
\hline 18 & 13 & 25 & 184 & 4 & 0 & 0 & 0 & 0 \\
17 & 38 & 25 & 151 & 8 & 0 & 53 & 0 & 0 & 0 \\
16 & 63 & 25 & 147 & 8 & 0 & 0 & 0 & 0 & 0 \\
15 & 88 & 25 & 140 & 2 & 0 & 0 & 0 & 0 & 0 \\
14 & 113 & 25 & 128 & 2 & 0 & 0 & 0 & 0 & 0 \\
13 & 138 & 25 & 125 & 2 & 0 & 16 & 0 & 0 & 0 \\
12 & 163 & 25 & 116 & 1 & 0 & 9 & 9 & 0 & 0 \\
11 & 188 & 25 & 164 & 1 & 0 & 0 & 0 & 0 & 0 \\
8 & 250 & 100 & 752 & 4 & 0 & 5 & 32 & 0 & 0 \\
7 & 350 & 100 & 662 & 2 & 0 & 0 & 3 & 0 & 0 \\
6 & 450 & 100 & 489 & 1 & 2 & 10 & 12 & 0 & 0 \\
5 & 550 & 100 & 514 & 4 & 0 & 47 & 39 & 0 & 0 \\
4 & 650 & 100 & 858 & 4 & 14 & 877 & 336 & 0 & 14 \\
3 & 750 & 100 & 532 & 2 & 26 & 1698 & 635 & 0 & 15 \\
1 & 850 & 100 & 424 & 2 & 71 & 1925 & 887 & 24 & 24 \\
& 950 & 100 & 340 & 1 & 26 & 1017 & 479 & 15 & 29 \\
\hline
\end{tabular}

net\# \#ften \#mten \#cten \#fngr \#mngr \#fnro \#cneo \#fund \#mund

\begin{tabular}{|c|c|c|c|c|c|c|c|c|c|}
\hline 18 & 22 & 0 & 0 & 261 & 0 & 22 & 457 & 501 & 435 \\
\hline 17 & 0 & 0 & 53 & 0 & 0 & 0 & 318 & 212 & 159 \\
\hline 16 & 1033 & 0 & 1794 & 0 & 0 & 0 & 109 & 163 & 109 \\
\hline 15 & 371 & 186 & 1057 & 0 & 0 & 14 & 214 & 0 & 0 \\
\hline 14 & 47 & 31 & 47 & 0 & 0 & 16 & 62 & 0 & 0 \\
\hline 13 & 0 & 48 & 32 & 64 & 0 & 16 & 64 & 0 & 0 \\
\hline 12 & 0 & 0 & 9 & 17 & 0 & 0 & 17 & 0 & 0 \\
\hline 11 & 6 & 18 & 6 & 0 & 0 & 0 & 12 & 0 & 0 \\
\hline 8 & 0 & 0 & 0 & 11 & 0 & 0 & 0 & 0 & 0 \\
\hline 7 & 0 & 0 & 0 & 3 & 0 & 0 & 24 & 0 & 0 \\
\hline 6 & 0 & 0 & 0 & 0 & 37 & 0 & 21 & 0 & 0 \\
\hline 5 & 0 & 0 & 0 & 0 & 54 & 0 & 23 & 0 & 0 \\
\hline 4 & 0 & 0 & 0 & 0 & 14 & 0 & 0 & 0 & 0 \\
\hline 3 & 0 & 0 & 0 & 0 & 4 & 0 & 0 & 0 & 0 \\
\hline 2 & 0 & 0 & 0 & 0 & 0 & 0 & 0 & 0 & 0 \\
\hline 1 & 0 & 0 & 0 & 0 & 0 & 0 & 0 & 0 & 0 \\
\hline
\end{tabular}


c218.std WCR-82H MOCNESS tow copepods

$4 e-2$

net\# \#cund \#ccal \#fmin \#mmin \#cmin \#felg \#celg \#fcor \#mcor

\begin{tabular}{|c|c|c|c|c|c|c|c|c|c|}
\hline 18 & 5658 & 566 & 1197 & 544 & 1632 & 0 & 0 & 0 & 0 \\
\hline 17 & 4612 & 159 & 636 & 265 & 2492 & 0 & 0 & 0 & 0 \\
\hline 16 & 1467 & 544 & 544 & 326 & 1141 & 0 & 0 & 0 & 0 \\
\hline 15 & 14 & 400 & 0 & 14 & 0 & 0 & 0 & 0 & 0 \\
\hline 14 & 0 & 203 & 47 & 16 & 47 & 16 & 16 & 16 & 0 \\
\hline 13 & 0 & 112 & 16 & 0 & 0 & 0 & 0 & 16 & 0 \\
\hline 12 & 0 & 61 & 17 & 0 & 9 & 0 & 0 & 0 & 0 \\
\hline 11 & 0 & 55 & 0 & 0 & 18 & 0 & 0 & 0 & 6 \\
\hline 8 & 0 & 64 & 0 & 0 & 5 & 0 & 0 & 11 & 0 \\
\hline 7 & 3 & 6 & 0 & 0 & 0 & 0 & 0 & 6 & 0 \\
\hline 6 & 0 & 2 & 0 & 0 & 0 & 0 & 4 & 12 & 0 \\
\hline 5 & 0 & 0 & 0 & 0 & 0 & 0 & 62 & 101 & 8 \\
\hline 4 & 0 & 0 & 0 & 0 & 5 & 33 & 37 & 182 & 37 \\
\hline 3 & 0 & 0 & 0 & 0 & 0 & 180 & 38 & 98 & 15 \\
\hline 2 & 0 & 9 & 0 & 0 & 14 & 43 & 14 & 9 & 0 \\
\hline 1 & 0 & 3 & 6 & 0 & 3 & 12 & 18 & 12 & 0 \\
\hline
\end{tabular}

\begin{tabular}{|c|c|c|c|c|c|c|c|c|c|}
\hline net\# & \#ccor & \#fnas & \#cnas & \# fmey & \#facr & \# fprv & \#farc & \#ffur & \#fege \\
\hline 18 & 65 & 0 & 0 & 0 & 1567 & 239 & 1328 & 305 & 0 \\
\hline 17 & 53 & 0 & 0 & 53 & 742 & 265 & 1484 & 583 & 0 \\
\hline 16 & 0 & 0 & 0 & 0 & 380 & 109 & 1576 & 0 & 0 \\
\hline 15 & 10 & 0 & 0 & 0 & 0 & 14 & 671 & 43 & 43 \\
\hline 14 & 16 & 0 & 0 & 0 & 0 & 16 & 250 & 0 & 62 \\
\hline 13 & 0 & 0 & 0 & 0 & 0 & 0 & 128 & 0 & 0 \\
\hline 12 & 0 & 0 & 0 & 0 & 0 & 0 & 35 & 9 & 9 \\
\hline 11 & 12 & 0 & 0 & 0 & 0 & 6 & 24 & 0 & 6 \\
\hline 8 & 43 & 0 & 0 & 0 & 0 & 0 & 27 & 0 & 0 \\
\hline 7 & 85 & 0 & 0 & 0 & 0 & 0 & 0 & 0 & 0 \\
\hline 6 & 115 & 0 & 0 & 0 & 0 & 0 & 0 & 0 & 0 \\
\hline 5 & 412 & 0 & 0 & 0 & 0 & 0 & 0 & 0 & 0 \\
\hline 4 & 229 & 0 & 0 & 0 & 0 & 0 & 9 & 0 & 0 \\
\hline 3 & 56 & 0 & 8 & 0 & 0 & 0 & 0 & 0 & 0 \\
\hline 2 & 19 & 0 & 19 & 0 & 0 & 0 & 0 & 0 & 5 \\
\hline 1 & 15 & 6 & 6 & 0 & 0 & 0 & 0 & 0 & 3 \\
\hline
\end{tabular}


c218.std WCR-82H MOCNESS tow copepods

$4 e-3$

\begin{tabular}{|c|c|c|c|c|c|c|c|c|c|}
\hline net\# & \# feme & \#meme & \#fupl & \#mupl & \# fumj & $\# f \operatorname{chi}$ & \# fmed & \#mmed & \# fmar \\
\hline 18 & 44 & 0 & 283 & 174 & 0 & 0 & 87 & 0 & 87 \\
\hline 17 & 53 & 53 & 53 & 0 & 0 & 0 & 265 & 0 & 159 \\
\hline 16 & 54 & 0 & 0 & 0 & 0 & 0 & 272 & 0 & 54 \\
\hline 15 & 57 & 14 & 0 & 29 & 14 & 0 & 200 & 157 & 0 \\
\hline 14 & 16 & 16 & 78 & 0 & 0 & 16 & 31 & 16 & 0 \\
\hline 13 & 64 & 32 & 224 & 32 & 16 & 48 & 64 & 48 & 0 \\
\hline 12 & 9 & 9 & 35 & 0 & 9 & 26 & 9 & 9 & 0 \\
\hline 11 & 0 & 0 & 12 & 6 & 0 & 12 & 6 & 6 & 0 \\
\hline 8 & 0 & 0 & 11 & 0 & 0 & 5 & 5 & 0 & 0 \\
\hline 7 & 0 & 0 & 3 & 3 & 0 & 9 & 0 & 0 & 0 \\
\hline 6 & 0 & 0 & 2 & 0 & 0 & 4 & 2 & 6 & 0 \\
\hline 5 & 0 & 0 & 0 & 0 & 0 & 0 & 16 & 8 & 0 \\
\hline 4 & 37 & 0 & 19 & 23 & 0 & 19 & 33 & 0 & 0 \\
\hline 3 & 11 & 0 & 38 & 4 & 0 & 0 & 0 & 0 & 0 \\
\hline 2 & 5 & 0 & 28 & 9 & 5 & 5 & 0 & 0 & 9 \\
\hline 1 & 6 & 0 & 18 & 21 & 0 & 0 & 0 & 0 & 0 \\
\hline
\end{tabular}

\begin{tabular}{rrrrrrrrrr} 
net\# & \#mmar & \#fsco & \#msco & \#fbra & \#mbra & \#fdan & \#mdan & \#fluc & \#mluc \\
\hline 18 & 0 & 0 & 0 & 44 & 0 & 522 & 631 & 0 & 0 \\
17 & 159 & 0 & 0 & 53 & 0 & 159 & 53 & 0 & 0 \\
16 & 54 & 0 & 0 & 217 & 0 & 0 & 0 & 0 & 0 \\
15 & 0 & 0 & 14 & 86 & 29 & 57 & 0 & 0 & 0 \\
14 & 0 & 31 & 16 & 16 & 0 & 0 & 0 & 0 & 0 \\
13 & 0 & 32 & 16 & 48 & 0 & 16 & 0 & 0 & 0 \\
12 & 0 & 9 & 9 & 9 & 17 & 9 & 0 & 0 & 0 \\
11 & 0 & 6 & 6 & 0 & 0 & 0 & 0 & 0 & 0 \\
8 & 0 & 11 & 0 & 0 & 0 & 0 & 0 & 0 & 0 \\
7 & 0 & 3 & 0 & 3 & 0 & 0 & 0 & 0 & 0 \\
6 & 0 & 4 & 0 & 2 & 0 & 0 & 2 & 0 & 0 \\
5 & 0 & 0 & 0 & 8 & 0 & 0 & 0 & 0 & 0 \\
4 & 0 & 0 & 0 & 0 & 0 & 0 & 0 & 0 & 0 \\
3 & 0 & 4 & 0 & 0 & 0 & 4 & 0 & 0 & 4 \\
2 & 0 & 0 & 0 & 0 & 0 & 19 & 0 & 0 & 5 \\
1 & 0 & 0 & 0 & 0 & 0 & 0 & 0 & 12 & 65 \\
\hline
\end{tabular}


c218.std WCR-82H MOCNESS tow copepods

$4 e-4$

net\# \#fven \#mven \#fbrv \#mbrv \#cmet \#fgra \#mgra \#fpis \#mpis

\begin{tabular}{rrrrrrrrrr}
\hline 18 & 0 & 0 & 0 & 0 & 0 & 392 & 44 & 566 & 0 \\
17 & 0 & 0 & 0 & 0 & 0 & 7210 & 1219 & 1166 & 848 \\
16 & 0 & 0 & 0 & 0 & 0 & 1196 & 1359 & 163 & 380 \\
15 & 0 & 0 & 0 & 0 & 0 & 57 & 214 & 57 & 0 \\
14 & 0 & 0 & 0 & 0 & 16 & 47 & 31 & 0 & 0 \\
13 & 0 & 0 & 16 & 16 & 0 & 64 & 16 & 16 & 0 \\
12 & 0 & 0 & 0 & 0 & 0 & 9 & 9 & 0 & 0 \\
11 & 0 & 0 & 0 & 0 & 0 & 12 & 12 & 0 & 0 \\
8 & 0 & 0 & 0 & 0 & 0 & 5 & 0 & 0 & 0 \\
7 & 0 & 0 & 0 & 0 & 0 & 0 & 0 & 0 & 0 \\
6 & 0 & 2 & 0 & 0 & 0 & 8 & 25 & 0 & 0 \\
5 & 16 & 23 & 8 & 0 & 148 & 23 & 78 & 31 & 47 \\
4 & 65 & 205 & 168 & 42 & 378 & 0 & 5 & 5 & 5 \\
3 & 252 & 68 & 90 & 143 & 90 & 0 & 4 & 0 & 0 \\
2 & 66 & 0 & 24 & 19 & 123 & 0 & 0 & 0 & 0 \\
1 & 0 & 0 & 26 & 12 & 394 & 0 & 0 & 0 & 0 \\
\hline
\end{tabular}

net\# \#cpsm \#fabd \#mabd \#fxip \#mxip \#cplg \#fvio \#mvio \#ftyp

\begin{tabular}{rrrrrrrrrr}
\hline 18 & 871 & 479 & 22 & 0 & 0 & 653 & 501 & 392 & 109 \\
17 & 4082 & 318 & 106 & 0 & 0 & 4559 & 371 & 106 & 795 \\
16 & 2174 & 435 & 489 & 0 & 0 & 2446 & 54 & 54 & 217 \\
15 & 100 & 86 & 171 & 186 & 129 & 857 & 0 & 0 & 29 \\
14 & 62 & 16 & 0 & 265 & 156 & 265 & 0 & 16 & 0 \\
13 & 32 & 32 & 32 & 1010 & 160 & 16 & 0 & 0 & 32 \\
12 & 17 & 0 & 17 & 95 & 26 & 17 & 0 & 9 & 0 \\
11 & 12 & 0 & 0 & 12 & 18 & 6 & 6 & 6 & 55 \\
8 & 16 & 0 & 0 & 0 & 0 & 0 & 0 & 5 & 11 \\
7 & 82 & 0 & 0 & 6 & 0 & 223 & 0 & 0 & 0 \\
6 & 241 & 0 & 0 & 0 & 0 & 141 & 0 & 0 & 4 \\
5 & 171 & 0 & 23 & 0 & 0 & 249 & 0 & 0 & 0 \\
4 & 9 & 9 & 33 & 5 & 47 & 149 & 0 & 0 & 0 \\
3 & 0 & 34 & 8 & 26 & 34 & 105 & 0 & 0 & 0 \\
2 & 0 & 0 & 14 & 0 & 5 & 19 & 0 & 0 & 9 \\
1 & 0 & 0 & 0 & 0 & 3 & 0 & 0 & 0 & 0 \\
\hline
\end{tabular}


c218.std WCR-82H MOCNESS tow copepods

$4 e-5$

\begin{tabular}{rrrrrrrrrr} 
net\# & \#mtyp & \#luci & \#fpap & \#mpap & \#fspi & \#mspi & \#fvip & \#mip & \#fhal \\
\hline 18 & 174 & 5310 & 65 & 87 & 0 & 0 & 0 & 0 & 22 \\
17 & 477 & 6044 & 212 & 265 & 0 & 0 & 0 & 0 & 0 \\
16 & 163 & 3478 & 489 & 326 & 0 & 0 & 0 & 0 & 978 \\
15 & 14 & 2429 & 157 & 100 & 14 & 0 & 0 & 0 & 571 \\
14 & 78 & 1482 & 94 & 125 & 0 & 62 & 0 & 0 & 140 \\
13 & 48 & 1010 & 385 & 289 & 64 & 80 & 0 & 0 & 497 \\
12 & 0 & 268 & 156 & 78 & 17 & 26 & 0 & 0 & 363 \\
11 & 24 & 580 & 92 & 18 & 12 & 0 & 0 & 0 & 342 \\
8 & 16 & 617 & 48 & 21 & 16 & 0 & 16 & 5 & 346 \\
7 & 3 & 296 & 36 & 6 & 6 & 3 & 12 & -18 & 731 \\
6 & 0 & 147 & 59 & 66 & 2 & 0 & 21 & 12 & 334 \\
5 & 0 & 47 & 47 & 0 & 8 & 16 & 16 & 23 & 140 \\
4 & 0 & 65 & 28 & 9 & 23 & 5 & 14 & 23 & 14 \\
3 & 0 & 86 & 0 & 0 & 4 & 0 & 19 & 8 \\
1 & 14 & 57 & 0 & 0 & 0 & 0 & 5 & 0 \\
\end{tabular}

net\# \#mhal \#chal \#fhel \#mhel \#fket \#mket \#fclo \#mclo \#fpak

\begin{tabular}{|c|c|c|c|c|c|c|c|c|c|c|}
\hline 18 & $\cdots$ & 0 & 22 & 0 & 22 & 0 & 0 & 0 & 0 & 22 \\
\hline 17 & & 0 & 53 & 0 & 0 & 0 & 0 & 0 & 0 & 0 \\
\hline 16 & & 0 & 0 & 0 & 0 & 0 & 0 & 54 & 0 & 54 \\
\hline 15 & & 43 & 471 & 14 & 0 & 0 & 0 & 57 & 14 & 0 \\
\hline 14 & & 16 & 1389 & 0 & 78 & 0 & 0 & 31 & 62 & 0 \\
\hline 13 & & 0 & 4824 & 96 & 112 & 0 & 0 & 32 & 48 & 0 \\
\hline 12 & & 9 & 2111 & 35 & 26 & 9 & 9 & 0 & 0 & 0 \\
\hline 11 & & 0 & 2254 & 37 & 43 & 0 & 0 & 0 & 0 & 0 \\
\hline 8 & & 0 & 1607 & 32 & 43 & 0 & 5 & 0 & 0 & 0 \\
\hline 7 & & 15 & 311 & 6 & 0 & 0 & 3 & 0 & 0 & 0 \\
\hline 6 & & 2 & 176 & 10 & 4 & 0 & 0 & 6 & 0 & 0 \\
\hline 5 & & 0 & 78 & 23 & 0 & 0 & 0 & 0 & 0 & 0 \\
\hline 4 & & 0 & 0 & 89 & 89 & 0 & 0 & 0 & 0 & 0 \\
\hline 3 & & 0 & 0 & 41 & 49 & 0 & 0 & 0 & 0 & 0 \\
\hline 2 & & 0 & 5 & 0 & 5 & 0 & 0 & 0 & 0 & 0 \\
\hline 1 & & $0:$ & 0 & 0 & 3 & 0 & 0 & 0 & 0 & 0 \\
\hline
\end{tabular}


c218.std WCR-82H MOCNESS tow copepods

$4 e-6$

net\# \#mpak \#fpbi \#mpbi \#mpsi \#acar \#fmis \#mmis \#cmis \#cycl

\begin{tabular}{rrrrrrrrrr}
\hline 18 & 0 & 152 & 131 & 0 & 2242 & 936 & 261 & 10424 & 11382 \\
17 & 53 & 106 & 159 & 0 & 1272 & 1007 & 477 & 15958 & 10179 \\
16 & 54 & 544 & 544 & 0 & 815 & 1685 & 109 & 11413 & 8478 \\
15 & 0 & 43 & 71 & 0 & 100 & 1014 & 171 & 4514 & 4571 \\
14 & 0 & 0 & 0 & 0 & 94 & 530 & 218 & 3604 & 3994 \\
13 & 0 & 0 & 0 & 16 & 16 & 433 & 96 & 3414 & 1699 \\
12 & 0 & 0 & 0 & 0 & 35 & 320 & 87 & 1557 & 614 \\
11 & 0 & 0 & 0 & 0 & 18 & 159 & 55 & 1295 & 751 \\
8 & 0 & 0 & 0 & 0 & 5 & 218 & 91 & 1256 & 559 \\
7 & 0 & 0 & 0 & 0 & 3 & 257 & 73 & 851 & 335 \\
6 & 0 & 0 & 0 & 0 & 2 & 305 & 96 & 1287 & 117 \\
5 & 0 & 0 & 0 & 0 & 0 & 817 & 218 & 2606 & 638 \\
4 & 0 & 0 & 0 & 0 & 0 & 928 & 406 & 2113 & 266 \\
3 & 0 & 0 & 0 & 0 & 0 & 451 & 109 & 1255 & 68 \\
2 & 0 & 0 & 0 & 0 & 0 & 415 & 85 & 1307 & 142 \\
1 & 0 & 0 & 0 & 0 & 6 & 303 & 53 & 1064 & 100 \\
\hline
\end{tabular}

\begin{tabular}{rrr} 
net\# & \#harp & \#tot 1 \\
\hline 18 & 479 & 52405 \\
17 & 318 & 70510 \\
16 & 272 & 48370 \\
15 & 14 & 19986 \\
14 & 0 & 14009 \\
13 & 16 & 15753 \\
12 & 0 & 6272 \\
11 & 6 & 6054 \\
8 & 11 & 5178 \\
7 & 6 & 3433 \\
6 & 0 & 3308 \\
5 & 16 & 6284 \\
4 & 28 & 7142 \\
3 & 4 & 5804 \\
2 & 0 & 5460 \\
1 & 12 & 3781 \\
\hline
\end{tabular}


c218.int WCR-82H MOCNESS tow copepods

$4 f-1$

net\# midd depi volf aliq iffin ivfin icfin ifhyp ichyp

\begin{tabular}{rrrrrrrrrr}
\hline 18 & 13 & 25 & 184 & 4 & 0 & 0 & 0 & 0 & 0 \\
17 & 38 & 25 & 151 & 8 & 0 & 1 & 0 & 0 & 0 \\
16 & 63 & 25 & 147 & 8 & 0 & 1 & 0 & 0 & 0 \\
15 & 88 & 25 & 140 & 2 & 0 & 1 & 0 & 0 & 0 \\
14 & 113 & 25 & 128 & 2 & 0 & 1 & 0 & 0 & 0 \\
13 & 138 & 25 & 125 & 2 & 0 & 2 & 0 & 0 & 0 \\
12 & 163 & 25 & 116 & 1 & 0 & 2 & 0 & 0 & 0 \\
11 & 188 & 25 & 164 & 1 & 0 & 2 & 0 & 0 & 0 \\
8 & 250 & 100 & 752 & 4 & 0 & 2 & 3 & 0 & 0 \\
7 & 350 & 100 & 662 & 2 & 0 & 2 & 4 & 0 & 0 \\
6 & 450 & 100 & 489 & 1 & 0 & 3 & 5 & 0 & 0 \\
5 & 550 & 100 & 514 & 4 & 0 & 8 & 9 & 0 & 0 \\
4 & 650 & 100 & 858 & 4 & 2 & 96 & 42 & 0 & 1 \\
3 & 750 & 100 & 532 & 2 & 4 & 265 & 106 & 0 & 3 \\
2 & 850 & 100 & 424 & 2 & 11 & 458 & 195 & 2 & 5 \\
1 & 950 & 100 & 340 & 1 & 14 & 560 & 243 & 4 & 8 \\
\hline
\end{tabular}

net\# iften imten icten ifngr imngr ifnro icneo ifund imund

\begin{tabular}{llllllllll}
\hline 18 & 1 & 0 & 0 & 7 & 0 & 1 & 11 & 13 & 11 \\
17 & 1 & 0 & 1 & 7 & 0 & 1 & 19 & 18 & 15 \\
16 & 26 & 0 & 46 & 7 & 0 & 1 & 22 & 22 & 18 \\
15 & 36 & 5 & 73 & 7 & 0 & 1 & 27 & 22 & 18 \\
14 & 37 & 5 & 74 & 7 & 0 & 1 & 29 & 22 & 18 \\
13 & 37 & 7 & 75 & 8 & 0 & 2 & 31 & 22 & 18 \\
12 & 37 & 7 & 75 & 9 & 0 & 2 & 31 & 22 & 18 \\
11 & 37 & 7 & 75 & 9 & 0 & 2 & 31 & 22 & 18 \\
8 & 37 & 7 & 75 & 10 & 0 & 2 & 31 & 22 & 18 \\
7 & 37 & 7 & 75 & 10 & 0 & 2 & 34 & 22 & 18 \\
6 & 37 & 7 & 75 & 10 & 4 & 2 & 36 & 22 & 18 \\
5 & 37 & 7 & 75 & 10 & 9 & 2 & 38 & 22 & 18 \\
4 & 37 & 7 & 75 & 10 & 11 & 2 & 38 & 22 & 18 \\
3 & 37 & 7 & 75 & 10 & 11 & 2 & 38 & 22 & 18 \\
2 & 37 & 7 & 75 & 10 & 11 & 2 & 38 & 22 & 18 \\
1 & 37 & 7 & 75 & 10 & 11 & 2 & 38 & 22 & 18 \\
\hline
\end{tabular}


c218.int WCR-82H MOCNESS tow copepods

$4 f-2$

\begin{tabular}{|c|c|c|c|c|c|c|c|c|c|}
\hline net\# & icund & iccal & ifmin & immin & icmin & ifelg & icelg & ifcor & imcor \\
\hline 18 & 141 & 14 & 30 & 14 & 41 & 0 & 0 & 0 & 0 \\
\hline 17 & 257 & 18 & 46 & 20 & 103 & 0 & 0 & 0 & 0 \\
\hline 16 & 294 & 32 & 59 & 28 & 132 & 0 & 0 & 0 & 0 \\
\hline 15 & 294 & 42 & 59 & 29 & 132 & 0 & 0 & 0 & 0 \\
\hline 14 & 294 & 47 & 61 & 29 & 133 & 0 & 0 & 0 & 0 \\
\hline 13 & 294 & 50 & 61 & 29 & 133 & 0 & 0 & 1 & 0 \\
\hline 12 & 294 & 51 & 61 & 29 & 133 & 0 & 0 & 1 & 0 \\
\hline 11 & 294 & 52 & 61 & 29 & 134 & 0 & 0 & 1 & 0 \\
\hline 8 & 294 & 59 & 61 & 29 & 134 & 0 & 0 & 2 & 0 \\
\hline 7 & 294 & 59 & 61 & 29 & 134 & 0 & 0 & 2 & 0 \\
\hline 6 & 294 & 60 & 61 & 29 & 134 & 0 & 1 & 4 & 0 \\
\hline 5 & 294 & 60 & 61 & 29 & 134 & 0 & 7 & 14 & 1 \\
\hline 4 & 294 & 60 & 61 & 29 & 135 & 4 & 11 & 32 & 5 \\
\hline 3 & 294 & 60 & 61 & 29 & 135 & 22 & 15 & 42 & 6 \\
\hline 2 & 294 & 61 & 61 & 29 & 136 & 26 & 16 & 43 & 6 \\
\hline 1 & 294 & 61 & 62 & 29 & 136 & 27 & 18 & 44 & 6 \\
\hline
\end{tabular}

\begin{tabular}{|c|c|c|c|c|c|c|c|c|c|}
\hline net\# & iccor & ifnas & icnas & ifmey & ifacr & ifprv & ifarc & iffur & ifege \\
\hline 18 & 2 & 0 & 0 & 0 & 39 & 6 & 33 & 8 & 0 \\
\hline 17 & 3 & 0 & 0 & 1 & 58 & 13 & 70 & 22 & 0 \\
\hline 16 & 3 & 0 & 0 & 1 & 67 & 15 & 110 & 22 & 0 \\
\hline 15 & 3 & 0 & 0 & 1 & 67 & 16 & 126 & 23 & 1 \\
\hline 14 & 3 & 0 & 0 & 1 & 67 & 16 & 133 & 23 & 3 \\
\hline 13 & 3 & 0 & 0 & 1 & 67 & 16 & 136 & 23 & 3 \\
\hline 12 & 3 & 0 & 0 & 1 & 67 & 16 & 137 & 23 & 3 \\
\hline 11 & 4 & 0 & 0 & 1 & 67 & 16 & 137 & 23 & 3 \\
\hline 8 & 8 & 0 & 0 & 1 & 67 & 16 & 140 & 23 & 3 \\
\hline 7 & 16 & 0 & 0 & 1 & 67 & 16 & 140 & 23 & 3 \\
\hline 6 & 28 & 0 & 0 & 1 & 67 & 16 & 140 & 23 & 3 \\
\hline 5 & 69 & 0 & 0 & 1 & 67 & 16 & 140 & 23 & 3 \\
\hline 4 & 92 & 0 & 0 & 1 & 67 & 16 & 141 & 23 & 3 \\
\hline 3 & 98 & 0 & 1 & 1 & 67 & 16 & 141 & 23 & 3 \\
\hline 2 & 100 & 0 & 3 & 1 & 67 & 16 & 141 & 23 & 3 \\
\hline 1 & 101 & 1 & 3 & 1 & 67 & 16 & 141 & 23 & 4 \\
\hline
\end{tabular}


c218.int WCR-82H MOCNESS tow copepods

$4 f-3$

\begin{tabular}{rrrrrrrrrr} 
net\# & ifeme & imeme & ifupl & imupl & ifumj & ifchi & ifmed & immed & ifmar \\
\hline 18 & 1 & 0 & 7 & 4 & 0 & 0 & 2 & 0 & 2 \\
17 & 2 & 1 & 8 & 4 & 0 & 0 & 9 & 0 & 6 \\
16 & 4 & 1 & 8 & 4 & 0 & 0 & 16 & 0 & 8 \\
15 & 5 & 2 & 8 & 5 & 0 & 0 & 21 & 4 & 8 \\
14 & 6 & 2 & 10 & 5 & 0 & 0 & 21 & 4 & 8 \\
13 & 7 & 3 & 16 & 6 & 1 & 2 & 23 & 6 & 8 \\
12 & 7 & 3 & 17 & 6 & 1 & 2 & 23 & 6 & 8 \\
11 & 7 & 3 & 17 & 6 & 1 & 3 & 23 & 6 & 8 \\
8 & 7 & 3 & 18 & 6 & 1 & 3 & 24 & 6 & 8 \\
7 & 7 & 3 & 19 & 6 & 1 & 4 & 24 & 6 & 8 \\
6 & 7 & 3 & 19 & 6 & 1 & 4 & 24 & 7 & 8 \\
5 & 7 & 3 & 19 & 6 & 1 & 4 & 26 & 7 & 8 \\
4 & 11 & 3 & 21 & 9 & 1 & 6 & 29 & 7 & 8 \\
3 & 12 & 3 & 24 & 9 & 1 & 6 & 29 & 7 & 8 \\
2 & 13 & 3 & 27 & 10 & 1 & 7 & 29 & 7 & 8 \\
1 & 13 & 3 & 29 & 12 & 1 & 7 & 29 & 7 & 8 \\
\hline
\end{tabular}

\begin{tabular}{rrrrrrrrrr} 
net\# & immar & ifsco & imsco & ifbra & imbra & ifdan & imdan & ifluc & imluc \\
\hline 18 & 0 & 0 & 0 & 1 & 0 & 13 & 16 & 0 & 0 \\
17 & 4 & 0 & 0 & 2 & 0 & 17 & 17 & 0 & 0 \\
16 & 5 & 0 & 0 & 8 & 0 & 17 & 17 & 0 & 0 \\
15 & 5 & 0 & 0 & 10 & 1 & 18 & 17 & 0 & 0 \\
14 & 5 & 1 & 1 & 10 & 1 & 18 & 17 & 0 & 0 \\
13 & 5 & 2 & 1 & 12 & 1 & 19 & 17 & 0 & 0 \\
12 & 5 & 2 & 1 & 12 & 1 & 19 & 17 & 0 & 0 \\
11 & 5 & 2 & 2 & 12 & 1 & 19 & 17 & 0 & 0 \\
8 & 5 & 3 & 2 & 12 & 1 & 19 & 17 & 0 & 0 \\
7 & 5 & 3 & 2 & 12 & 1 & 19 & 17 & 0 & 0 \\
6 & 5 & 4 & 2 & 12 & 1 & 19 & 17 & 0 & 0 \\
5 & 5 & 4 & 2 & 13 & 1 & 19 & 17 & 0 & 0 \\
4 & 5 & 4 & 2 & 13 & 1 & 19 & 17 & 0 & 0 \\
3 & 5 & 4 & 2 & 13 & 1 & 19 & 17 & 0 & 0 \\
2 & 5 & 4 & 2 & 13 & 1 & 21 & 17 & 0 & 1 \\
1 & 5 & 4 & 2 & 13 & 1 & 21 & 17 & 1 & 7 \\
\hline
\end{tabular}


c218.int WCR-82H MOCNESS tow copepods

$4 f-4$

\begin{tabular}{crrrrrrrrr} 
net\# & ifven & imven & ifbrv & imbrv & icmet & ifgra & imgra & ifpis & impis \\
\hline 18 & 0 & 0 & 0 & 0 & 0 & 10 & 1 & 14 & 0 \\
17 & 0 & 0 & 0 & 0 & 0 & 190 & 32 & 43 & 21 \\
16 & 0 & 0 & 0 & 0 & 0 & 220 & 66 & 47 & 31 \\
15 & 0 & 0 & 0 & 0 & 0 & 221 & 71 & 49 & 31 \\
14 & 0 & 0 & 0 & 0 & 0 & 222 & 72 & 49 & 31 \\
13 & 0 & 0 & 0 & 0 & 0 & 224 & 72 & 49 & 31 \\
12 & 0 & 0 & 0 & 0 & 0 & 224 & 72 & 49 & 31 \\
11 & 0 & 0 & 0 & 0 & 0 & 224 & 73 & 49 & 31 \\
8 & 0 & 0 & 0 & 0 & 0 & 225 & 73 & 49 & 31 \\
7 & 0 & 0 & 0 & 0 & 0 & 225 & 73 & 49 & 31 \\
6 & 0 & 0 & 0 & 0 & 0 & 226 & 75 & 49 & 31 \\
5 & 2 & 3 & 1 & 0 & 15 & 228 & 83 & 52 & 35 \\
4 & 8 & 23 & 18 & 5 & 53 & 228 & 83 & 53 & 36 \\
3 & 33 & 30 & 27 & 19 & 62 & 228 & 84 & 53 & 36 \\
2 & 40 & 30 & 29 & 21 & 74 & 228 & 84 & 53 & 36 \\
1 & 40 & 30 & 32 & 22 & 114 & 228 & 84 & 53 & 36 \\
\hline
\end{tabular}

net\# icpsm ifabd imabd ifxip imxip icplg ifvio imvio iftyp

\begin{tabular}{rrrrrrrrrr}
\hline 18 & 22 & 12 & 1 & 0 & 0 & 16 & 13 & 10 & 3 \\
17 & 124 & 20 & 3 & 0 & 0 & 130 & 22 & 12 & 23 \\
16 & 178 & 31 & 15 & 0 & 0 & 192 & 23 & 14 & 28 \\
15 & 181 & 33 & 20 & 5 & 3 & 213 & 23 & 14 & 29 \\
14 & 182 & 33 & 20 & 11 & 7 & 220 & 23 & 14 & 29 \\
13 & 183 & 34 & 21 & 37 & 11 & 220 & 23 & 14 & 30 \\
12 & 184 & 34 & 21 & 39 & 12 & 220 & 23 & 14 & 30 \\
11 & 184 & 34 & 21 & 39 & 12 & 220 & 23 & 15 & 31 \\
8 & 185 & 34 & 21 & 39 & 12 & 220 & 23 & 15 & 32 \\
7 & 194 & 34 & 21 & 40 & 12 & 242 & 23 & 15 & 32 \\
6 & 218 & 34 & 21 & 40 & 12 & 257 & 23 & 15 & 32 \\
5 & 235 & 34 & 23 & 40 & 12 & 282 & 23 & 15 & 32 \\
4 & 236 & 35 & 27 & 40 & 17 & 297 & 23 & 15 & 32 \\
3 & 236 & 38 & 27 & 43 & 20 & 307 & 23 & 15 & 32 \\
2 & 236 & 38 & 29 & 43 & 21 & 309 & 23 & 15 & 33 \\
1 & 236 & 38 & 29 & 43 & 21 & 309 & 23 & 15 & 33 \\
\hline
\end{tabular}


c218.int WCR-82H MOCNESS tow copepods

$4 f-5$

\begin{tabular}{rrrrrrrrrr} 
net\# & imtyp & iluci & ifpap & impap & ifspi & imspi & ifvip & imvip & ifhal \\
\hline 18 & 4 & 133 & 2 & 2 & 0 & 0 & 0 & 0 & 0 \\
17 & 16 & 284 & 7 & 9 & 0 & 0 & 0 & 0 & 0 \\
16 & 20 & 371 & 19 & 17 & 0 & 0 & 0 & 0 & 25 \\
15 & 21 & 432 & 23 & 19 & 0 & 0 & 0 & 0 & 39 \\
14 & 23 & 469 & 25 & 23 & 0 & 2 & 0 & 0 & 43 \\
13 & 24 & 494 & 35 & 30 & 2 & 4 & 0 & 0 & 55 \\
12 & 24 & 500 & 39 & 32 & 2 & 4 & 0 & 0 & 64 \\
11 & 24 & 515 & 41 & 32 & 3 & 4 & 0 & 0 & 73 \\
8 & 26 & 576 & 46 & 34 & 4 & 4 & 2 & 1 & 107 \\
7 & 26 & 606 & 50 & 35 & 5 & 5 & 3 & 2 & 180 \\
6 & 26 & 621 & 56 & 41 & 5 & 5 & 5 & 4 & 214 \\
5 & 26 & 625 & 60 & 41 & 6 & 6 & 6 & 6 & 228 \\
4 & 26 & 632 & 63 & 42 & 8 & 7 & 8 & 8 & 229 \\
3 & 26 & 640 & 63 & 42 & 9 & 7 & 10 & 9 & 229 \\
2 & 28 & 646 & 63 & 42 & 9 & 7 & 10 & 9 & 229 \\
1 & 29 & 647 & 63 & 42 & 9 & 7 & 10 & 9 & 230 \\
\hline
\end{tabular}

net\# imhal ichal ifhel imhel ifket imket ifclo imclo ifpak

\begin{tabular}{rrrrrrrrrr}
\hline 18 & 0 & 0 & 0 & 1 & 0 & 0 & 0 & 0 & 1 \\
17 & 0 & 2 & 0 & 1 & 0 & 0 & 0 & 0 & 1 \\
16 & 0 & 2 & 0 & 1 & 0 & 0 & 1 & 0 & 2 \\
15 & 1 & 14 & 0 & 1 & 0 & 0 & 3 & 0 & 2 \\
14 & 1 & 49 & 0 & 2 & 0 & 0 & 4 & 2 & 2 \\
13 & 1 & 169 & 3 & 5 & 0 & 0 & 4 & 3 & 2 \\
12 & 2 & 222 & 4 & 6 & 0 & 0 & 4 & 3 & 2 \\
11 & 2 & 278 & 5 & 7 & 0 & 0 & 4 & 3 & 2 \\
8 & 2 & 439 & 8 & 11 & 0 & 1 & 4 & 3 & 2 \\
7 & 3 & 470 & 8 & 11 & 0 & 1 & 4 & 3 & 2 \\
6 & 3 & 487 & 9 & 12 & 0 & 1 & 5 & 3 & 2 \\
5 & 3 & 495 & 12 & 12 & 0 & 1 & 5 & 3 & 2 \\
4 & 3 & 495 & 20 & 21 & 0 & 1 & 5 & 3 & 2 \\
3 & 3 & 495 & 25 & 25 & 0 & 1 & 5 & 3 & 2 \\
2 & 3 & 496 & 25 & 26 & 0 & 1 & 5 & 3 & 2 \\
1 & 3 & 496 & 25 & 26 & 0 & 1 & 5 & 3 & 2 \\
\hline
\end{tabular}


c218.int WCR-82H MOCNESS tow copepods

4f- 6

\begin{tabular}{rrrrrrrrrr} 
net\# & impak & ifpbi & impbi & impsi & iacar & ifmis & immis & icmis & icycl \\
\hline 18 & 0 & 4 & 3 & 0 & 56 & 23 & 7 & 260 & 284 \\
17 & 1 & 6 & 7 & 0 & 88 & 48 & 19 & 659 & 538 \\
16 & 3 & 20 & 21 & 0 & 108 & 91 & 21 & 944 & 751 \\
15 & 3 & 21 & 23 & 0 & 111 & 116 & 26 & 1058 & 865 \\
14 & 3 & 21 & 23 & 0 & 113 & 129 & 31 & 1147 & 964 \\
13 & 3 & 21 & 23 & 0 & 113 & 140 & 33 & 1232 & 1007 \\
12 & 3 & 21 & 23 & 0 & 114 & 148 & 36 & 1271 & 1023 \\
11 & 3 & 21 & 23 & 0 & 115 & 152 & 37 & 1304 & 1042 \\
8 & 3 & 21 & 23 & 0 & 115 & 174 & 46 & 1430 & 1097 \\
7 & 3 & 21 & 23 & 0 & 115 & 200 & 53 & 1514 & 1131 \\
6 & 3 & 21 & 23 & 0 & 116 & 230 & 63 & 1643 & 1143 \\
5 & 3 & 21 & 23 & 0 & 116 & 312 & 84 & 1903 & 1206 \\
4 & 3 & 21 & 23 & 0 & 116 & 405 & 125 & 2114 & 1232 \\
3 & 3 & 21 & 23 & 0 & 116 & 449 & 136 & 2240 & 1239 \\
2 & 3 & 21 & 23 & 0 & 116 & 491 & 144 & 2372 & 1253 \\
1 & 3 & 21 & 23 & 0 & 116 & 521 & 150 & 2478 & 1263 \\
\hline
\end{tabular}

\begin{tabular}{rrr} 
net\# & iharp & itotl \\
\hline 18 & 12 & 1313 \\
17 & 20 & 3080 \\
16 & 27 & 4285 \\
15 & 27 & 4788 \\
14 & 27 & 5134 \\
13 & 27 & 5529 \\
12 & 27 & 5687 \\
11 & 28 & 5835 \\
8 & 29 & 6348 \\
7 & 29 & 6694 \\
6 & 29 & 7030 \\
5 & 31 & 7661 \\
4 & 34 & 8372 \\
3 & 34 & 8955 \\
2 & 34 & 9498 \\
1 & 35 & 9873 \\
\hline
\end{tabular}


c218. cum WCR-82H MOCNESS tow copepods

$4 g-1$

\begin{tabular}{rrrrrrrrrr} 
net\# & midd & depi & volf & aliq & offin & ovfin & ocfin & ofhyp & \%chyp \\
\hline 18 & 13 & 25 & 184 & 4 & 0 & 0 & 0 & 0 & 0 \\
17 & 38 & 25 & 151 & 8 & 0 & 0 & 0 & 0 & 0 \\
16 & 63 & 25 & 147 & 8 & 0 & 0 & 0 & 0 & 0 \\
15 & 88 & 25 & 140 & 2 & 0 & 0 & 0 & 0 & 0 \\
14 & 113 & 25 & 128 & 2 & 0 & 0 & 0 & 0 & 0 \\
13 & 138 & 25 & 125 & 2 & 0 & 0 & 0 & 0 & 0 \\
12 & 163 & 25 & 116 & 1 & 0 & 0 & 0 & 0 & 0 \\
11 & 188 & 25 & 164 & 1 & 0 & 0 & 0 & 0 & 0 \\
8 & 250 & 100 & 752 & 4 & 0 & 0 & 1 & 0 & 0 \\
7 & 350 & 100 & 662 & 2 & 0 & 0 & 2 & 0 & 0 \\
6 & 450 & 100 & 489 & 1 & 1 & 1 & 2 & 0 & 0 \\
5 & 550 & 100 & 514 & 4 & 1 & 1 & 4 & 0 & 0 \\
4 & 650 & 100 & 858 & 4 & 11 & 17 & 17 & 0 & 17 \\
3 & 750 & 100 & 532 & 2 & 30 & 47 & 44 & 0 & 35 \\
2 & 850 & 100 & 424 & 2 & 81 & 82 & 80 & 62 & 64 \\
1 & 950 & 100 & 340 & 1 & 100 & 100 & 100 & 100 & 100 \\
\hline
\end{tabular}

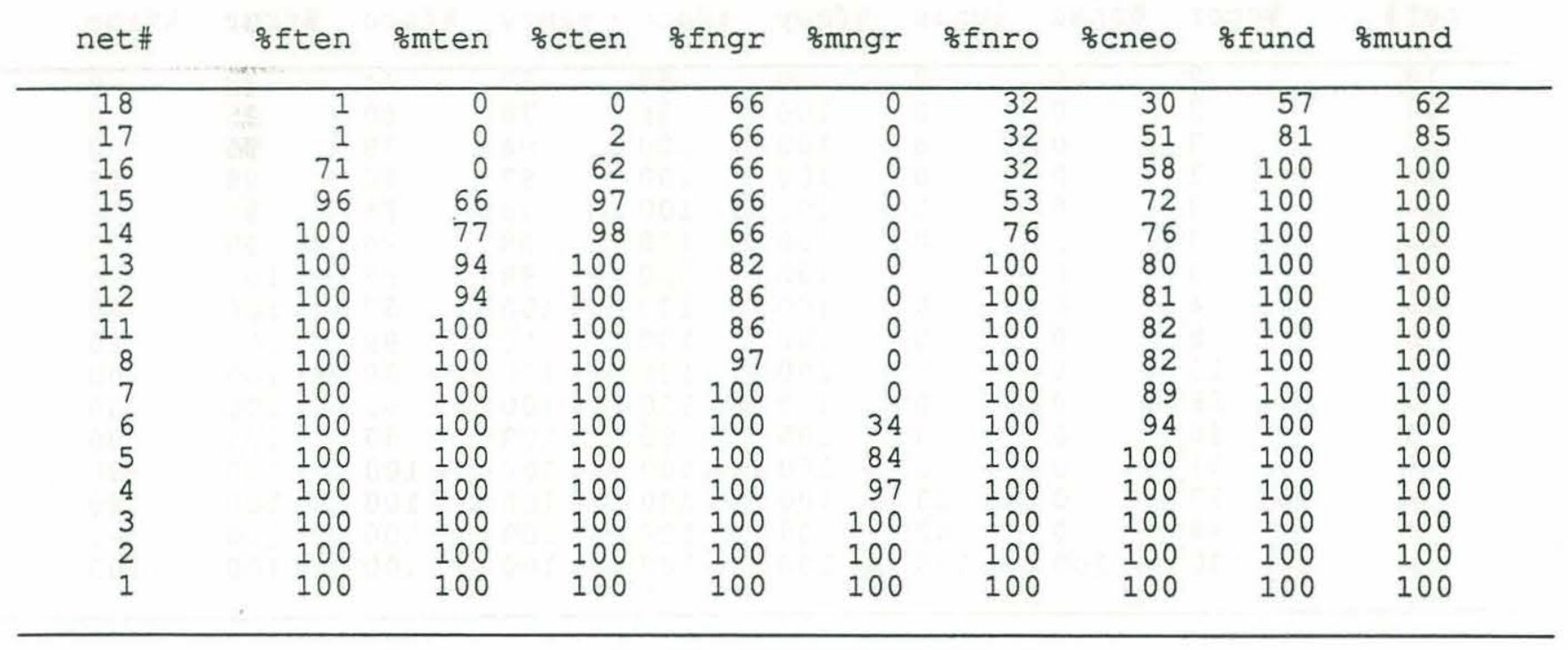


c218. cum WCR-82H MOCNESS tow copepods

$4 g-2$

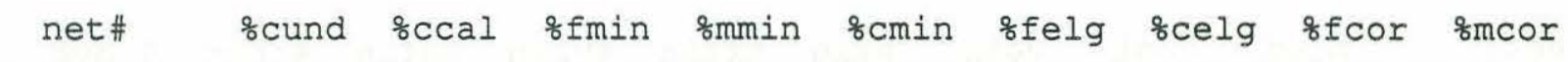

\begin{tabular}{rrrrrrrrrr}
\hline 18 & 48 & 23 & 48 & 47 & 30 & 0 & 0 & 0 & 0 \\
17 & 87 & 30 & 74 & 69 & 76 & 0 & 0 & 0 & 0 \\
16 & 100 & 52 & 96 & 97 & 97 & 0 & 0 & 0 & 0 \\
15 & 100 & 68 & 96 & 99 & 97 & 0 & 0 & 0 & 0 \\
14 & 100 & 77 & 98 & 100 & 98 & 1 & 2 & 1 & 0 \\
13 & 100 & 81 & 98 & 100 & 98 & 1 & 2 & 2 & 0 \\
12 & 100 & 84 & 99 & 100 & 98 & 1 & 2 & 2 & 0 \\
11 & 100 & 86 & 99 & 100 & 98 & 1 & 2 & 2 & 2 \\
8 & 100 & 97 & 99 & 100 & 98 & 1 & 2 & 4 & 2 \\
7 & 100 & 98 & 99 & 100 & 98 & 1 & 2 & 6 & 2 \\
6 & 100 & 98 & 99 & 100 & 98 & 1 & 5 & 8 & 2 \\
5 & 100 & 98 & 99 & 100 & 98 & 1 & 40 & 31 & 15 \\
4 & 100 & 98 & 99 & 100 & 99 & 13 & 61 & 73 & 76 \\
3 & 100 & 98 & 99 & 100 & 99 & 80 & 82 & 95 & 100 \\
2 & 100 & 100 & 99 & 100 & 100 & 96 & 90 & 97 & 100 \\
1 & 100 & 100 & 100 & 100 & 100 & 100 & 100 & 100 & 100 \\
\hline
\end{tabular}

\begin{tabular}{rrrrrrrrrr} 
net\# & ofccor & offnas & ofnas & ofmey & ofacr & ofprv & ofarc & offur & ofege \\
\hline 18 & 2 & 0 & 0 & 0 & 58 & 37 & 24 & 32 & 0 \\
17 & 3 & 0 & 0 & 100 & 86 & 78 & 50 & 95 & 0 \\
16 & 3 & 0 & 0 & 100 & 100 & 94 & 78 & 95 & 0 \\
15 & 3 & 0 & 0 & 100 & 100 & 97 & 90 & 99 & 29 \\
14 & 3 & 0 & 0 & 100 & 100 & 99 & 94 & 99 & 70 \\
13 & 3 & 0 & 0 & 100 & 100 & 99 & 96 & 99 & 70 \\
12 & 3 & 0 & 0 & 100 & 100 & 99 & 97 & 100 & 76 \\
11 & 4 & 0 & 0 & 100 & 100 & 100 & 97 & 100 & 80 \\
8 & 8 & 0 & 0 & 100 & 100 & 100 & 99 & 100 & 80 \\
7 & 16 & 0 & 0 & 100 & 100 & 100 & 99 & 100 & 80 \\
6 & 28 & 0 & 0 & 100 & 100 & 100 & 99 & 100 & 80 \\
5 & 68 & 0 & 0 & 100 & 100 & 100 & 99 & 100 & 80 \\
4 & 91 & 0 & 0 & 100 & 100 & 100 & 100 & 100 & 80 \\
3 & 97 & 0 & 23 & 100 & 100 & 100 & 100 & 100 & 80 \\
2 & 99 & 0 & 82 & 100 & 100 & 100 & 100 & 100 & 92 \\
1 & 100 & 100 & 100 & 100 & 100 & 100 & 100 & 100 & 100 \\
\hline
\end{tabular}


c218. cum WCR-82H MOCNESS tow copepods

$4 g-3$

net\# \%feme smeme ofupl smupl ofumj ofchi ofmed ommed ofmar

\begin{tabular}{rrrrrrrrrr}
\hline 18 & 8 & 0 & 24 & 36 & 0 & 0 & 8 & 0 & 26 \\
17 & 18 & 43 & 29 & 36 & 0 & 0 & 30 & 0 & 73 \\
16 & 28 & 43 & 29 & 36 & 0 & 0 & 54 & 0 & 89 \\
15 & 39 & 54 & 29 & 42 & 25 & 0 & 71 & 54 & 89 \\
14 & 42 & 67 & 36 & 42 & 25 & 6 & 74 & 59 & 89 \\
13 & 54 & 93 & 55 & 49 & 52 & 24 & 79 & 76 & 89 \\
12 & 56 & 100 & 58 & 49 & 67 & 33 & 80 & 79 & 89 \\
11 & 56 & 100 & 59 & 50 & 67 & 38 & 81 & 81 & 89 \\
8 & 56 & 100 & 63 & 50 & 67 & 46 & 83 & 81 & 89 \\
7 & 56 & 100 & 64 & 53 & 67 & 59 & 83 & 81 & 89 \\
6 & 56 & 100 & 65 & 53 & 67 & 65 & 83 & 89 & 89 \\
5 & 56 & 100 & 65 & 53 & 67 & 65 & 89 & 100 & 89 \\
4 & 84 & 100 & 71 & 72 & 67 & 93 & 100 & 100 & 89 \\
3 & 92 & 100 & 84 & 75 & 67 & 93 & 100 & 100 & 89 \\
2 & 96 & 100 & 94 & 83 & 100 & 100 & 100 & 100 & 100 \\
1 & 100 & 100 & 100 & 100 & 100 & 100 & 100 & 100 & 100 \\
\hline
\end{tabular}

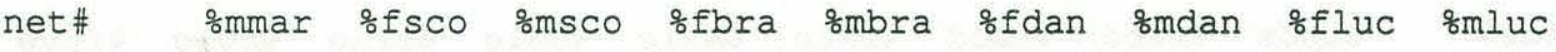

\begin{tabular}{rrrrrrrrrr}
\hline 18 & 0 & 0 & 0 & 8 & 0 & 61 & 91 & 0 & 0 \\
17 & 75 & 0 & 0 & 18 & 0 & 80 & 99 & 0 & 0 \\
16 & 100 & 0 & 0 & 60 & 0 & 80 & 99 & 0 & 0 \\
15 & 100 & 0 & 24 & 76 & 62 & 86 & 99 & 0 & 0 \\
14 & 100 & 19 & 49 & 79 & 62 & 86 & 99 & 0 & 0 \\
13 & 100 & 39 & 76 & 89 & 62 & 88 & 99 & 0 & 0 \\
12 & 100 & 44 & 90 & 90 & 100 & 89 & 99 & 0 & 0 \\
11 & 100 & 48 & 100 & 90 & 100 & 89 & 99 & 0 & 0 \\
8 & 100 & 73 & 100 & 90 & 100 & 89 & 99 & 0 & 0 \\
7 & 100 & 81 & 100 & 93 & 100 & 89 & 99 & 0 & 0 \\
6 & 100 & 91 & 100 & 94 & 100 & 89 & 100 & 0 & 0 \\
5 & 100 & 91 & 100 & 100 & 100 & 89 & 100 & 0 & 0 \\
4 & 100 & 91 & 100 & 100 & 100 & 89 & 100 & 0 & 0 \\
3 & 100 & 100 & 100 & 100 & 100 & 91 & 100 & 0 & 5 \\
2 & 100 & 100 & 100 & 100 & 100 & 100 & 100 & 0 & 12 \\
1 & 100 & 100 & 100 & 100 & 100 & 100 & 100 & 100 & 100 \\
\hline
\end{tabular}


c218. cum WCR-82H MOCNESS tow copepods

$4 \mathrm{~g} \cdot 4$

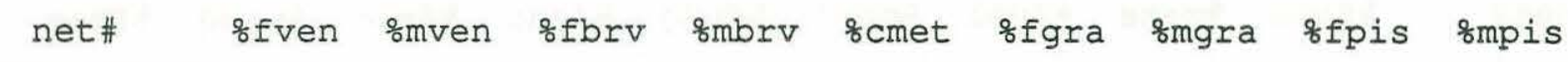

\begin{tabular}{rrrrrrrrrr}
\hline 18 & 0 & 0 & 0 & 0 & 0 & 4 & 1 & 27 & 0 \\
17 & 0 & 0 & 0 & 0 & 0 & 83 & 38 & 82 & 59 \\
16 & 0 & 0 & 0 & 0 & 0 & 96 & 78 & 90 & 86 \\
15 & 0 & 0 & 0 & 0 & 0 & 97 & 85 & 92 & 86 \\
14 & 0 & 0 & 0 & 0 & 0 & 97 & 86 & 92 & 86 \\
13 & 0 & 0 & 1 & 2 & 0 & 98 & 86 & 93 & 86 \\
12 & 0 & 0 & 1 & 2 & 0 & 98 & 86 & 93 & 86 \\
11 & 0 & 0 & 1 & 2 & 0 & 98 & 87 & 93 & 86 \\
8 & 0 & 0 & 1 & 2 & 0 & 99 & 87 & 93 & 86 \\
7 & 0 & 0 & 1 & 2 & 0 & 99 & 87 & 93 & 86 \\
6 & 0 & 1 & 1 & 2 & 0 & 99 & 90 & 93 & 86 \\
5 & 4 & 8 & 4 & 2 & 13 & 100 & 99 & 99 & 99 \\
4 & 20 & 77 & 56 & 21 & 47 & 100 & 100 & 100 & 100 \\
3 & 83 & 100 & 84 & 86 & 55 & 100 & 100 & 100 & 100 \\
2 & 100 & 100 & 92 & 95 & 65 & 100 & 100 & 100 & 100 \\
1 & 100 & 100 & 100 & 100 & 100 & 100 & 100 & 100 & 100 \\
\hline
\end{tabular}

net\# scpsm sfabd smabd sfxip smxip scplg sfvio smvio sftyp

\begin{tabular}{rrrrrrrrrr}
\hline 18 & 9 & 31 & 2 & 0 & 0 & 5 & 54 & 65 & 8. \\
17 & 53 & 52 & 11 & 0 & 0 & 42 & 94 & 82 & 68 \\
16 & 76 & 80 & 54 & 0 & 0 & 62 & 99 & 91 & 84 \\
15 & 77 & 86 & 69 & 11 & 15 & 69 & 99 & 91 & 86 \\
14 & 77 & 87 & 69 & 26 & 34 & 71 & 99 & 94 & 86 \\
13 & 78 & 89 & 71 & 85 & 53 & 71 & 99 & 94 & 89 \\
12 & 78 & 89 & 73 & 91 & 56 & 71 & 99 & 95 & 89 \\
11 & 78 & 89 & 73 & 91 & 58 & 71 & 100 & 96 & 93 \\
8 & 79 & 89 & 73 & 91 & 58 & 71 & 100 & 100 & 96 \\
7 & 82 & 89 & 73 & 93 & 58 & 79 & 100 & 100 & 96 \\
6 & 92 & 89 & 73 & 93 & 58 & 83 & 100 & 100 & 97 \\
5 & 100 & 89 & 81 & 93 & 58 & 91 & 100 & 100 & 97 \\
4 & 100 & 91 & 92 & 94 & 80 & 96 & 100 & 100 & 97 \\
3 & 100 & 100 & 95 & 100 & 96 & 99 & 100 & 100 & 97 \\
2 & 100 & 100 & 100 & 100 & 99 & 100 & 100 & 100 & 100 \\
1 & 100 & 100 & 100 & 100 & 100 & 100 & 100 & 100 & 100 \\
\hline
\end{tabular}


c218. cum WCR-82H MOCNESS tow copepods

$4 g \cdot 5$

net\# \&mtyp \&luci \&fpap \&mpap \&fspi \&mspi \&fvip \&mvip \&fhal

\begin{tabular}{rrrrrrrrrr}
\hline 18 & 15 & 20 & 3 & 5 & 0 & 0 & 0 & 0 & 0 \\
17 & 56 & 44 & 11 & 21 & 0 & 0 & 0 & 0 & 0 \\
16 & 70 & 57 & 30 & 40 & 0 & 0 & 0 & 0 & 11 \\
15 & 71 & 67 & 37 & 46 & 4 & 0 & 0 & 0 & 17 \\
14 & 77 & 72 & 40 & 53 & 4 & 24 & 0 & 0 & 19 \\
13 & 82 & 76 & 56 & 70 & 23 & 54 & 0 & 0 & 24 \\
12 & 82 & 77 & 62 & 75 & 28 & 64 & 0 & 0 & 28 \\
11 & 84 & 79 & 65 & 76 & 31 & 64 & 0 & 0 & 32 \\
8 & 89 & 89 & 73 & 81 & 50 & 64 & 16 & 6 & 47 \\
7 & 90 & 94 & 79 & 82 & 57 & 69 & 28 & 26 & 79 \\
6 & 90 & 96 & 88 & 98 & 59 & 69 & 48 & 40 & 93 \\
5 & 90 & 97 & 96 & 98 & 68 & 93 & 63 & 66 & 99 \\
4 & 90 & 98 & 100 & 100 & 96 & 100 & 77 & 92 & 100 \\
3 & 90 & 99 & 100 & 100 & 100 & 100 & 95 & 100 & 100 \\
2 & 95 & 100 & 100 & 100 & 100 & 100 & 100 & 100 & 100 \\
1 & 100 & 100 & 100 & 100 & 100 & 100 & 100 & 100 & 100 \\
\hline
\end{tabular}

net\# \&mhal schal sfhel smhel sfket smket \%fclo smclo sfpak

\begin{tabular}{rrrrrrrrrr}
\hline 18 & 0 & 0 & 0 & 2 & 0 & 0 & 0 & 0 & 29 \\
17 & 0 & 0 & 0 & 2 & 0 & 0 & 0 & 0 & 29 \\
16 & 0 & 0 & 0 & 2 & 0 & 0 & 27 & 0 & 100 \\
15 & 32 & 3 & 1 & 2 & 0 & 0 & 56 & 11 & 100 \\
14 & 43 & 10 & 1 & 10 & 0 & 0 & 72 & 61 & 100 \\
13 & 43 & 34 & 11 & 20 & 0 & 0 & 88 & 100 & 100 \\
12 & 50 & 45 & 15 & 23 & 100 & 21 & 88 & 100 & 100 \\
11 & 50 & 56 & 18 & 27 & 100 & 21 & 88 & 100 & 100 \\
8 & 50 & 89 & 31 & 43 & 100 & 71 & 88 & 100 & 100 \\
7 & 94 & 95 & 34 & 43 & 100 & 100 & 88 & 100 & 100 \\
6 & 100 & 98 & 38 & 45 & 100 & 100 & 100 & 100 & 100 \\
5 & 100 & 100 & 47 & 45 & 100 & 100 & 100 & 100 & 100 \\
4 & 100 & 100 & 83 & 78 & 100 & 100 & 100 & 100 & 100 \\
3 & 100 & 100 & 100 & 97 & 100 & 100 & 100 & 100 & 100 \\
2 & 100 & 100 & 100 & 99 & 100 & 100 & 100 & 100 & 100 \\
1 & 100 & 100 & 100 & 100 & 100 & 100 & 100 & 100 & 100 \\
\hline
\end{tabular}


C218. cum WCR-82H MOCNESS tow copepods

$4 g \cdot 6$

net\# \&mpak \%fpbi \&mpbi smpsi \&acar \&fmis \&mmis \%cmis \%cycl

\begin{tabular}{rrrrrrrrrr}
\hline 18 & 0 & 18 & 14 & 0 & 48 & 4 & 4 & 11 & 23 \\
17 & 49 & 31 & 32 & 0 & 75 & 9 & 12 & 27 & 43 \\
16 & 100 & 95 & 92 & 0 & 93 & 17 & 14 & 38 & 59 \\
15 & 100 & 100 & 100 & 0 & 95 & 22 & 17 & 43 & 68 \\
14 & 100 & 100 & 100 & 0 & 97 & 25 & 21 & 46 & 76 \\
13 & 100 & 100 & 100 & 100 & 97 & 27 & 22 & 50 & 80 \\
12 & 100 & 100 & 100 & 100 & 98 & 28 & 24 & 51 & 81 \\
11 & 100 & 100 & 100 & 100 & 99 & 29 & 25 & 53 & 82 \\
8 & 100 & 100 & 100 & 100 & 99 & 33 & 31 & 58 & 87 \\
7 & 100 & 100 & 100 & 100 & 99 & 38 & 35 & 61 & 89 \\
6 & 100 & 100 & 100 & 100 & 99 & 44 & 42 & 66 & 90 \\
5 & 100 & 100 & 100 & 100 & 99 & 60 & 56 & 77 & 95 \\
4 & 100 & 100 & 100 & 100 & 99 & 78 & 84 & 85 & 98 \\
3 & 100 & 100 & 100 & 100 & 99 & 86 & 91 & 90 & 98 \\
2 & 100 & 100 & 100 & 100 & 99 & 94 & 96 & 96 & 99 \\
1 & 100 & 100 & 100 & 100 & 100 & 100 & 100 & 100 & 100 \\
\hline
\end{tabular}

\begin{tabular}{rrr} 
net\# & oharp & stotl \\
\hline 18 & 34 & 13 \\
17 & 57 & 31 \\
16 & 76 & 43 \\
15 & 77 & 48 \\
14 & 77 & 52 \\
13 & 78 & 56 \\
12 & 78 & 58 \\
11 & 78 & 59 \\
8 & 81 & 64 \\
7 & 83 & 68 \\
6 & 83 & 71 \\
5 & 88 & 78 \\
4 & 96 & 85 \\
3 & 97 & 91 \\
2 & 97 & 96 \\
1 & 100 & 100 \\
\hline
\end{tabular}


c219.raw WCR-82H MOCNESS tow copepods

$4 h-1$

\begin{tabular}{|c|c|c|c|c|c|c|c|c|c|}
\hline net\# & midd & depi & volf & aliq & ffin & mfin & vfin & cfin & fhyp \\
\hline 18 & 13 & 25 & 221 & 4 & 0 & 0 & 0 & 0 & 0 \\
\hline 17 & 38 & 25 & 206 & 4 & 0 & 0 & 0 & 0 & 0 \\
\hline 16 & 63 & 25 & 135 & 4 & 0 & 0 & 0 & 0 & 0 \\
\hline 15 & 88 & 25 & 130 & 2 & 0 & 0 & 0 & 0 & 0 \\
\hline 14 & 113 & 25 & 130 & 1 & 0 & 0 & 0 & 0 & 0 \\
\hline 13 & 138 & 25 & 153 & 1 & 0 & 0 & 0 & 0 & 0 \\
\hline 12 & 163 & 25 & 181 & 1 & 0 & 0 & 0 & 0 & 0 \\
\hline 11 & 188 & 25 & 204 & 1 & 0 & 0 & 0 & 0 & 0 \\
\hline 8 & 250 & 100 & 559 & 4 & 0 & 0 & 0 & 0 & 0 \\
\hline 7 & 350 & 100 & 567 & 4 & 0 & 0 & 1 & 0 & 0 \\
\hline 6 & 450 & 100 & 507 & 4 & 0 & 0 & 1 & 1 & 0 \\
\hline 5 & 550 & 100 & 495 & 2 & 0 & 0 & 2 & 1 & 0 \\
\hline 4 & 650 & 100 & 494 & 4 & 0 & 0 & 2 & 1 & 0 \\
\hline 3 & 750 & 100 & 823 & 4 & 4 & 0 & 92 & 36 & 0 \\
\hline 2 & 850 & 100 & 430 & 2 & 5 & 1 & 227 & 68 & 3 \\
\hline 1 & 950 & 100 & 424 & 2 & 5 & 0 & 222 & 99 & 2 \\
\hline
\end{tabular}

\begin{tabular}{|c|c|c|c|c|c|c|c|c|c|}
\hline net\# & chyp & ften & mten & cten & fngr & mngr & cngr & fund & mund \\
\hline 18 & 0 & 2 & 0 & 2 & 0 & 0 & 7 & 16 & 25 \\
\hline 17 & 0 & 12 & 0 & 6 & 2 & 0 & 18 & 1 & 3 \\
\hline 16 & 0 & 29 & 7 & 39 & 0 & 0 & 5 & 1 & 0 \\
\hline 15 & 0 & 27 & 28 & 67 & 1 & 0 & 1 & 0 & 0 \\
\hline 14 & 0 & 19 & 10 & 4 & 0 & 0 & 1 & 0 & 0 \\
\hline 13 & 0 & 6 & 11 & 1 & 0 & 0 & 3 & 0 & 0 \\
\hline 12 & 0 & 1 & 8 & 1 & 0 & 0 & 15 & 0 & 0 \\
\hline 11 & 0 & 0 & 0 & 0 & 1 & 0 & 26 & 0 & 0 \\
\hline 8 & 0 & 0 & 0 & 0 & 1 & 0 & 13 & 0 & 0 \\
\hline 7 & 0 & 0 & 0 & 0 & 0 & 0 & 14 & 0 & 0 \\
\hline 6 & 0 & 0 & 0 & 0 & 2 & 0 & 10 & 0 & 0 \\
\hline 5 & 1 & 0 & 0 & 0 & 2 & 7 & 2 & 0 & 0 \\
\hline 4 & 1 & 0 & 0 & 0 & 1 & 6 & 1 & 0 & 0 \\
\hline 3 & 1 & 0 & 0 & 0 & 1 & 7 & 0 & 0 & 0 \\
\hline 2 & 3 & 0 & 0 & 0 & 0 & 0 & 0 & 0 & 0 \\
\hline 1 & - 10 & 0 & 0 & 0 & 0 & 0 & 1 & 0 & 1 \\
\hline
\end{tabular}


C219.raw WCR-82H MOCNESS tow copepods

$4 h-2$

\begin{tabular}{rrrrrrrrrr} 
net\# & cund & ccal & fmin & mmin & cmin & fatt & fmon & felg & fcor \\
\hline 18 & 135 & 18 & 26 & 11 & 88 & 1 & 0 & 0 & 0 \\
17 & 10 & 21 & 26 & 11 & 69 & 1 & 0 & 0 & 0 \\
16 & 0 & 34 & 21 & 11 & 11 & 3 & 2 & 0 & 0 \\
15 & 0 & 10 & 13 & 5 & 3 & 1 & 0 & 0 & 0 \\
14 & 0 & 27 & 4 & 3 & 1 & 0 & 0 & 0 & 0 \\
13 & 0 & 13 & 1 & 0 & 0 & 0 & 0 & 0 & 1 \\
12 & 0 & 25 & 2 & 0 & 0 & 0 & 0 & 0 & 1 \\
11 & 0 & 6 & 0 & 0 & 0 & 0 & 1 & 0 & 0 \\
8 & 0 & 0 & 0 & 0 & 0 & 0 & 0 & 0 & 0 \\
7 & 0 & 0 & 0 & 0 & 1 & 0 & 0 & 0 & 2 \\
6 & 0 & 0 & 0 & 0 & 0 & 0 & 0 & 0 & 2 \\
5 & 0 & 0 & 1 & 0 & 1 & 0 & 0 & 0 & 14 \\
4 & 0 & 0 & 0 & 0 & 0 & 0 & 1 & 5 & 24 \\
3 & 0 & 0 & 0 & 0 & 0 & 2 & 1 & 19 & 48 \\
2 & 0 & 0 & 0 & 0 & 0 & 1 & 0 & 32 & 27 \\
1 & 0 & 0 & 0 & 0 & 1 & 3 & 0 & 12 & 7 \\
\hline
\end{tabular}

\begin{tabular}{rrrrrrrrrr} 
net\# & mcor & ccor & fmey & facr & fprv & farc & furc & mcla & fssp \\
\hline 18 & 0 & 0 & 0 & 28 & 1 & 53 & 1 & 3 & 0 \\
17 & 0 & 1 & 1 & 14 & 0 & 88 & 0 & 8 & 0 \\
16 & 0 & 1 & 1 & 7 & 6 & 8 & 0 & 0 & 0 \\
15 & 0 & 0 & 0 & 0 & 0 & 0 & 2 & 3 & 0 \\
14 & 0 & 1 & 0 & 0 & 0 & 2 & 1 & 0 & 0 \\
13 & 0 & 1 & 0 & 0 & 0 & 0 & 0 & 0 & 0 \\
12 & 0 & 0 & 0 & 0 & 0 & 0 & 0 & 0 & 0 \\
11 & 1 & 2 & 0 & 0 & 0 & 0 & 0 & 0 & 0 \\
8 & 0 & 2 & 0 & 0 & 0 & 6 & 0 & 0 & 0 \\
7 & 1 & 5 & 0 & 0 & 0 & 20 & 0 & 0 & 1 \\
6 & 0 & 9 & 0 & 0 & 0 & 6 & 0 & 0 & 10 \\
5 & 3 & 56 & 0 & 0 & 0 & 16 & 1 & 0 & 178 \\
4 & 2 & 52 & 0 & 0 & 0 & 4 & 0 & 0 & 68 \\
3 & 10 & 78 & 0 & 0 & 0 & 1 & 1 & 0 & 63 \\
2 & 2 & 19 & 0 & 0 & 0 & 0 & 0 & 0 & 21 \\
1 & 1 & 9 & 0 & 0 & 0 & 0 & 1 & 0 & 31 \\
\hline
\end{tabular}


c219.raw WCR-82H MOCNESS tow copepods

$4 h-3$

\begin{tabular}{|c|c|c|c|c|c|c|c|c|c|}
\hline net\# & fmim & fmty & faar & maar & fege & fgmi & feme & meme & fupl \\
\hline 18 & 0 & 0 & 0 & 0 & 0 & 0 & 0 & 0 & 0 \\
\hline 17 & 0 & 0 & 0 & 0 & 0 & 0 & 1 & 0 & 0 \\
\hline 16 & 0 & 0 & 0 & 0 & 25 & 0 & 0 & 0 & 0 \\
\hline 15 & 0 & 0 & 0 & 10 & 17 & 0 & 0 & 0 & 0 \\
\hline 14 & 0 & 0 & 0 & 9 & 1 & 0 & 0 & 0 & 0 \\
\hline 13 & 0 & 0 & 0 & 1 & 3 & 0 & 0 & 0 & 0 \\
\hline 12 & 0 & 0 & 0 & 3 & 3 & 0 & 0 & 0 & 0 \\
\hline 11 & 0 & 0 & 0 & 1 & 1 & 0 & 0 & 0 & 0 \\
\hline 8 & 0 & 0 & 0 & 0 & 2 & 0 & 0 & 0 & 0 \\
\hline 7 & 1 & 0 & 0 & 0 & 0 & 0 & 0 & 0 & 0 \\
\hline 6 & 5 & 0 & 0 & 0 & 0 & 11 & 0 & 0 & 0 \\
\hline 5 & 6 & 0 & 0 & 0 & 0 & 3 & 4 & 0 & 2 \\
\hline 4 & 4 & .0 & 1 & 0 & 0 & 2 & 11 & 2 & 13 \\
\hline 3 & 3 & 0 & 3 & 0 & 0 & 2 & 7 & 4 & 12 \\
\hline 2 & 1 & 2 & 1 & 0 & 0 & 0 & 7 & 1 & 4 \\
\hline 1 & 3 & 6 & 0 & 0 & 0 & 0 & 3 & 0 & 3 \\
\hline
\end{tabular}

\begin{tabular}{|c|c|c|c|c|c|c|c|c|c|}
\hline net\# & mupl & fumj & fchi & fmed & mmed & fmar & mmar & finc & $\operatorname{minc}$ \\
\hline 18 & 0 & 0 & 0 & 0 & 0 & 3 & 1 & 0 & 0 \\
\hline 17 & 0 & 0 & 0 & 0 & 0 & 2 & 0 & 0 & 0 \\
\hline 16 & 0 & 0 & 0 & 0 & 0 & 3 & 1 & 0 & 0 \\
\hline 15 & 0 & 0 & 0 & 1 & 0 & 0 & 0 & 0 & 0 \\
\hline 14 & 0 & 0 & 0 & 0 & 0 & 0 & 0 & 0 & 0 \\
\hline 13 & 0 & 0 & 0 & 0 & 0 & 0 & 0 & 0 & 0 \\
\hline 12 & 0 & 0 & 0 & 1 & 0 & 0 & 0 & 0 & 0 \\
\hline 11 & 0 & 0 & 0 & 0 & 0 & 0 & 0 & 0 & 0 \\
\hline 8 & 0 & 0 & 0 & 1 & 0 & 0 & 0 & 0 & 0 \\
\hline 7 & 0 & 0 & 0 & 1 & 0 & 0 & 0 & 0 & 0 \\
\hline 6 & 1 & 0 & 0 & 2 & 3 & 0 & 0 & 0 & 0 \\
\hline 5 & 1 & 0 & 0 & 27 & 15 & 0 & 0 & 0 & 0 \\
\hline 4 & 3 & 2 & 2 & 6 & 2 & 0 & 0 & 0 & 0 \\
\hline 3 & 5 & 1 & 5 & 0 & 0 & 0 & 0 & 1 & 4 \\
\hline 2 & 2 & 0 & 2 & 0 & 0 & 0 & 0 & 0 & 1 \\
\hline 1 & 0 & 0 & 0 & 0 & 0 & 0 & 0 & 0 & 0 \\
\hline
\end{tabular}


c219.raw WCR-82H MOCNESS tow copepods

$4 h-4$

\begin{tabular}{|c|c|c|c|c|c|c|c|c|c|}
\hline net\# & ceuk & fpsp & fslo & fsam & fscu & fsma & $\mathrm{mscp}$ & fbra & mbra \\
\hline 18 & 230 & 0 & 0 & 1 & 0 & 0 & 0 & 0 & 0 \\
\hline 17 & 124 & 0 & 0 & 0 & 0 & 0 & 0 & 0 & 0 \\
\hline 16 & 30 & 0 & 0 & 0 & 0 & 0 & 0 & 0 & 0 \\
\hline 15 & 191 & 0 & 0 & 0 & 0 & 0 & 0 & 7 & 4 \\
\hline 14 & 174 & 2 & 0 & 0 & 0 & 0 & 0 & 3 & 0 \\
\hline 13 & 107 & 0 & 0 & 0 & 0 & 0 & 0 & 5 & 0 \\
\hline 12 & 67 & 0 & 0 & 0 & 0 & 0 & 0 & 2 & 1 \\
\hline 11 & 44 & 3 & 0 & 1 & 0 & 0 & 1 & 2 & 0 \\
\hline 8 & 30 & 0 & 0 & 1 & 0 & 0 & 0 & 0 & 0 \\
\hline 7 & 41 & 0 & 0 & 3 & 0 & 0 & 0 & 4 & 0 \\
\hline 6 & 19 & 0 & 10 & 61 & 10 & 0 & 8 & 0 & 1 \\
\hline 5 & 16 & 0 & 129 & 101 & 17 & 0 & 9 & 0 & 2 \\
\hline 4 & 6 & 0 & 12 & 6 & 6 & 1 & 2 & 0 & 0 \\
\hline 3 & 21 & 0 & 9 & 0 & 15 & 0 & 49 & 0 & 0 \\
\hline 2 & 8 & 0 & 11 & 0 & 0 & 1 & 4 & 0 & 0 \\
\hline 1 & 14 & 0 & 1 & 0 & 0 & 0 & 0 & 0 & 0 \\
\hline
\end{tabular}

\begin{tabular}{|c|c|c|c|c|c|c|c|c|c|}
\hline net\# & fdan & mdan & fden & fvit & $\mathrm{msca}$ & ftst & ftmt & fven & mven \\
\hline 18 & 10 & 1 & 0 & 0 & 0 & 1 & 0 & 0 & 0 \\
\hline 17 & 4 & 0 & 0 & 0 & 0 & 2 & 0 & 0 & 0 \\
\hline 16 & 0 & 0 & 0 & 0 & 2 & 1 & 0 & 0 & 0 \\
\hline 15 & 0 & 0 & 0 & 0 & 3 & 0 & 0 & 0 & 0 \\
\hline 14 & 0 & 0 & 1 & 3 & 5 & 0 & 0 & 0 & 0 \\
\hline 13 & 0 & 0 & 0 & 2 & 3 & 0 & 0 & 0 & 0 \\
\hline 12 & 0 & 0 & 2 & 1 & 3 & 0 & 0 & 0 & 0 \\
\hline 11 & 0 & 0 & 2 & 2 & 8 & 0 & 0 & 0 & 0 \\
\hline 8 & 0 & 0 & 0 & 2 & 1 & 0 & 0 & 0 & 0 \\
\hline 7 & 0 & 0 & 1 & 1 & 2 & 0 & 0 & 0 & 0 \\
\hline 6 & 0 & 0 & 7 & 0 & 9 & 0 & 0 & 0 & 0 \\
\hline 5 & 0 & 0 & 6 & 0 & 12 & 0 & 0 & 1 & 1 \\
\hline 4 & 0 & 0 & 3 & 0 & 29 & 0 & 0 & 2 & 1 \\
\hline 3 & 0 & 0 & 0 & 0 & 17 & 0 & 1 & 37 & 29 \\
\hline 2 & 0 & 0 & 0 & 0 & 0 & 0 & 1 & 27 & 10 \\
\hline 1 & 0 & 0 & 0 & 0 & 4 & 0 & 1 & 18 & 2 \\
\hline
\end{tabular}


c219.raw WCR-82H MOCNESS tow copepods

$4 n-5$

net\# fbrv mbrv mlon cmet fgra mgra fpis mpis cpsm

\begin{tabular}{rrrrrrrrrr}
\hline 18 & 0 & 0 & 0 & 0 & 0 & 0 & 0 & 0 & 0 \\
17 & 0 & 0 & 0 & 0 & 4 & 0 & 0 & 1 & 2 \\
16 & 0 & 0 & 0 & 0 & 0 & 0 & 0 & 0 & 0 \\
15 & 0 & 0 & 0 & 0 & 0 & 0 & 0 & 0 & 0 \\
14 & 0 & 1 & 0 & 0 & 1 & 2 & 2 & 0 & 4 \\
13 & 0 & 0 & 0 & 0 & 0 & 0 & 0 & 0 & 5 \\
12 & 0 & 0 & 0 & 0 & 0 & 0 & 0 & 0 & 16 \\
11 & 0 & 0 & 0 & 0 & 2 & 4 & 5 & 0 & 106 \\
8 & 0 & 0 & 0 & 0 & 121 & 38 & 64 & 30 & 152 \\
7 & 0 & 0 & 0 & 0 & 67 & 11 & 39 & 11 & 37 \\
6 & 0 & 0 & 0 & 0 & 37 & 67 & 0 & 1 & 30 \\
5 & 0 & 0 & 0 & 3 & 52 & 80 & 9 & 4 & 28 \\
4 & 0 & 0 & 0 & 59 & 2 & 1 & 7 & 9 & 14 \\
3 & 35 & 4 & 1 & 134 & 0 & 0 & 0 & 0 & 0 \\
2 & 20 & 39 & 1 & 7 & 0 & 0 & 0 & 0 & 0 \\
1 & 15 & 1 & 1 & 17 & 0 & 0 & 0 & 0 & 0 \\
\hline
\end{tabular}

\begin{tabular}{rrrrrrrrrr} 
net\# & fabd & mabd & fxip & mxip & cplg & fvio & mvio & flcl & mlcl \\
\hline 18 & 1 & 0 & 0 & 0 & 1 & 1 & 2 & 0 & 0 \\
17 & 1 & 0 & 0 & 0 & 16 & 0 & 1 & 0 & 0 \\
16 & 0 & 0 & 0 & 0 & 5 & 1 & 0 & 0 & 0 \\
15 & 0 & 0 & 0 & 0 & 23 & 0 & 0 & 0 & 0 \\
14 & 0 & 1 & 0 & 1 & 117 & 0 & 0 & 0 & 0 \\
13 & 0 & 0 & 1 & 0 & 32 & 0 & 0 & 1 & 0 \\
12 & 0 & 0 & 0 & 0 & 21 & 0 & 0 & 10 & 4 \\
11 & 1 & 0 & 0 & 0 & 24 & 0 & 0 & 21 & 8 \\
8 & 1 & 1 & 1 & 0 & 68 & 0 & 0 & 14 & 1 \\
7 & 15 & 1 & 0 & 1 & 199 & 0 & 0 & 45 & 45 \\
6 & 26 & 13 & 0 & 0 & 53 & 0 & 0 & 34 & 32 \\
5 & 32 & 13 & 122 & 23 & 45 & 0 & 1 & 0 & 0 \\
4 & 4 & 7 & 5 & 4 & 45 & 0 & 0 & 0 & 0 \\
3 & 3 & 1 & 5 & 3 & 53 & 0 & 0 & 0 & 0 \\
2 & 5 & 4 & 4 & 7 & 19 & 0 & 0 & 0 & 0 \\
1 & 0 & 5 & 1 & 0 & 6 & 0 & 0 & 0 & 0 \\
\hline
\end{tabular}


c219.raw WCR-82H MOCNESS tow copepods

$4 h-6$

\begin{tabular}{|c|c|c|c|c|c|c|c|c|c|}
\hline net\# & flut & mlut & fpap & mpap & fspi & mspi & fvip & mvip & fhet \\
\hline 18 & 7 & 2 & 7 & 5 & 0 & 0 & 0 & 0 & 0 \\
\hline 17 & 54 & 35 & 15 & 21 & 0 & 0 & 0 & 0 & 0 \\
\hline 16 & 217 & 130 & 6 & 10 & 0 & 0 & 0 & 0 & 0 \\
\hline 15 & 237 & 126 & 5 & 7 & 0 & 0 & 0 & 0 & 0 \\
\hline 14 & 162 & 54 & 7 & 5 & 0 & 0 & 0 & 0 & 0 \\
\hline 13 & 115 & 17 & 3 & 9 & 1 & 1 & 0 & 0 & 0 \\
\hline 12 & 143 & 8 & 6 & 9 & 0 & 0 & 0 & 0 & 0 \\
\hline 11 & 117 & 16 & 7 & 19 & 1 & 1 & 0 & 0 & 0 \\
\hline 8 & 75 & 20 & 4 & 7 & 0 & 0 & 0 & 0 & 0 \\
\hline 7 & 36 & 3 & 18 & 4 & 5 & 2 & 2 & 1 & 0 \\
\hline 6 & 17 & 0 & 25 & 5 & 0 & 2 & 3 & 3 & 4 \\
\hline 5 & 22 & 4 & 24 & 4 & 0 & 0 & 0 & 0 & 2 \\
\hline 4 & 2 & 1 & 3 & 0 & 3 & 0 & 3 & 1 & 1 \\
\hline 3 & 11 & 4 & 6 & 0 & 2 & 0 & 3 & 13 & 5 \\
\hline 2 & 4 & 10 & 1 & 0 & 0 & 0 & 6 & 0 & 12 \\
\hline 1 & 5 & 2 & 3 & 0 & 0 & 0 & 4 & 0 & 1 \\
\hline
\end{tabular}

\begin{tabular}{|c|c|c|c|c|c|c|c|c|c|}
\hline net\# & mhet & chet & fhal & mhal & chal & forn & faug & fhel & mhel \\
\hline 18 & 0 & 0 & 0 & 0 & 0 & 0 & 0 & 0 & 0 \\
\hline 17 & 0 & 19 & 0 & 0 & 0 & 0 & 0 & 0 & 0 \\
\hline 16 & 0 & 38 & 205 & 9 & 15 & 1 & 5 & 0 & 0 \\
\hline 15 & 0 & 20 & 34 & 7 & 112 & 2 & 0 & 0 & 0 \\
\hline 14 & 0 & 35 & 29 & 7 & 777 & 1 & 7 & 0 & 0 \\
\hline 13 & 0 & 13 & 27 & 3 & 396 & 0 & 8 & 0 & 0 \\
\hline 12 & 0 & 29 & 13 & 1 & 139 & 0 & 3 & 0 & 0 \\
\hline 11 & 0 & 62 & 6 & 0 & 200 & 0 & 0 & 0 & 0 \\
\hline 8 & 0 & 45 & 10 & 0 & 124 & 1 & 1 & 0 & 0 \\
\hline 7 & 0 & 37 & 70 & 1 & 24 & 1 & 21 & 0 & 0 \\
\hline 6 & 1 & 44 & 54 & 0 & 64 & 8 & 2 & 0 & 0 \\
\hline 5 & 0 & 20 & 48 & 0 & 36 & 17 & 7 & 8 & 6 \\
\hline 4 & 1 & 0 & 10 & 0 & 2 & 2 & 10 & 16 & 12 \\
\hline 3 & 7 & 21 & 3 & 0 & 0 & 0 & 42 & 17 & 5 \\
\hline 2 & 5 & 0 & 0 & 0 & 0 & 0 & 15 & 2 & 1 \\
\hline 1 & 1 & 6 & 0 & 0 & 1 & 0 & 4 & 2 & 2 \\
\hline
\end{tabular}


c219.raw WCR-82H MOCNESS tow copepods

$4 h-7$

net\# chel fket mket fclo mclo fpak mpak fpbi mpbi

\begin{tabular}{|c|c|c|c|c|c|c|c|c|c|}
\hline 18 & 0 & 0 & 0 & 0 & 0 & 0 & 2 & 0 & 5 \\
\hline 17 & 0 & 0 & 0 & 0 & 0 & 1 & 0 & 15 & 13 \\
\hline 16 & 0 & 0 & 0 & 0 & 0 & 0 & 0 & 12 & 6 \\
\hline 15 & 0 & 0 & 0 & 0 & 0 & 1 & 0 & 2 & 0 \\
\hline 14 & 0 & 0 & 0 & 0 & 0 & 0 & 0 & 1 & 0 \\
\hline 13 & 0 & 0 & 0 & 0 & 0 & 0 & 0 & 0 & 0 \\
\hline 12 & 0 & 0 & 0 & 0 & 0 & 0 & 0 & 0 & 1 \\
\hline 11 & 0 & 0 & 0 & 0 & 1 & 0 & 1 & 0 & 0 \\
\hline 8 & 0 & 0 & 0 & 0 & 0 & 0 & 0 & 0 & 0 \\
\hline 7 & 2 & 0 & 0 & 0 & 0 & 0 & 0 & 0 & 0 \\
\hline 6 & 17 & 4 & 1 & 0 & 0 & 0 & 0 & 1 & 0 \\
\hline 5 & 42 & 3 & 3 & 17 & 18 & 0 & 0 & 0 & 0 \\
\hline 4 & 12 & 0 & 0 & 0 & 0 & 1 & 0 & 0 & 0 \\
\hline 3 & 39 & 0 & 0 & 0 & 0 & 0 & 0 & 0 & 0 \\
\hline 2 & 1 & 0 & 0 & 0 & 0 & 0 & 0 & 0 & 0 \\
\hline 1 & 8 & 0 & 0 & 0 & 0 & 0 & 0 & 0 & 0 \\
\hline
\end{tabular}

\begin{tabular}{rrrrrrrrrr} 
net\# & fpon & fada & fneg & fmis & mmis & cmis & oith & harp totl \\
\hline 18 & 3 & 0 & 33 & 0 & 2 & 162 & 12 & 321 & 1230 \\
17 & 1 & 0 & 9 & 3 & 0 & 221 & 15 & 246 & 1118 \\
16 & 2 & 0 & 10 & 27 & 4 & 158 & 151 & 147 & 1407 \\
15 & 0 & 0 & 0 & 6 & 0 & 111 & 159 & 187 & 1433 \\
14 & 0 & 0 & 0 & 6 & 0 & 172 & 48 & 206 & 1917 \\
13 & 0 & 0 & 3 & 0 & 3 & 50 & 3 & 127 & 976 \\
12 & 0 & 1 & 1 & 3 & 1 & 62 & 6 & 88 & 701 \\
11 & 0 & 0 & 2 & 2 & 3 & 90 & 32 & 64 & 897 \\
8 & 0 & 0 & 0 & 1 & 3 & 94 & 6 & 28 & 969 \\
7 & 0 & 0 & 0 & 9 & 0 & 92 & 28 & 13 & 940 \\
6 & 0 & 0 & 0 & 5 & 5 & 413 & 21 & 18 & 1198 \\
5 & 0 & 1 & 0 & 10 & 2 & 462 & 7 & 66 & 1878 \\
4 & 0 & 0 & 0 & 9 & 2 & 217 & 3 & 80 & 832 \\
3 & 0 & 0 & 0 & 13 & 15 & 317 & 2 & 35 & 1387 \\
1 & 0 & 0 & 0 & 12 & 9 & 274 & 2 & 31 & 985 \\
& 0 & 0 & 1 & 23 & 3 & 216 & 0 & 25 & 812 \\
\hline
\end{tabular}


c219.std WCR-82H MOCNESS tow copepods

$4 i-1$

\begin{tabular}{rrrrrrrrrr} 
net\# & midd & depi & volf & aliq & \#ffin & \#mfin & \#vfin & \#cfin & \#fhyp \\
\hline 18 & 13 & 25 & 221 & 4 & 0 & 0 & 0 & 0 & 0 \\
17 & 38 & 25 & 206 & 4 & 0 & 0 & 0 & 0 & 0 \\
16 & 63 & 25 & 135 & 4 & 0 & 0 & 0 & 0 & 0 \\
15 & 88 & 25 & 130 & 2 & 0 & 0 & 0 & 0 & 0 \\
14 & 113 & 25 & 130 & 1 & 0 & 0 & 0 & 0 & 0 \\
13 & 138 & 25 & 153 & 1 & 0 & 0 & 0 & 0 & 0 \\
12 & 163 & 25 & 181 & 1 & 0 & 0 & 0 & 0 & 0 \\
11 & 188 & 25 & 204 & 1 & 0 & 0 & 0 & 0 & 0 \\
8 & 250 & 100 & 559 & 4 & 0 & 0 & 0 & 0 & 0 \\
7 & 350 & 100 & 567 & 4 & 0 & 0 & 7 & 0 & 0 \\
6 & 450 & 100 & 507 & 4 & 0 & 0 & 8 & 8 & 0 \\
5 & 550 & 100 & 495 & 2 & 0 & 0 & 8 & 4 & 0 \\
4 & 650 & 100 & 494 & 4 & 0 & 0 & 16 & 8 & 0 \\
3 & 750 & 100 & 823 & 4 & 19 & 0 & 447 & 175 & 0 \\
2 & 850 & 100 & 430 & 2 & 23 & 5 & 1056 & 316 & 14 \\
1 & 950 & 100 & 424 & 2 & 24 & 0 & 1048 & 467 & 9 \\
\hline
\end{tabular}

net\# \#chyp \#ften \#mten \#cten \#fngr \#mngr \#cngr \#fund \#mund

\begin{tabular}{rrrrrrrrrr}
\hline 18 & 0 & 36 & 0 & 36 & 0 & 0 & 127 & 290 & 453 \\
17 & 0 & 234 & 0 & 117 & 39 & 0 & 350 & 20 & 58 \\
16 & 0 & 859 & 207 & 1156 & 0 & 0 & 148 & 30 & 0 \\
15 & 0 & 415 & 431 & 1031 & 15 & 0 & 15 & 0 & 0 \\
14 & 0 & 146 & 77 & 31 & 0 & 0 & 8 & 0 & 0 \\
13 & 0 & 39 & 72 & 7 & 0 & 0 & 20 & 0 & 0 \\
12 & 0 & 6 & 44 & 6 & 0 & 0 & 83 & 0 & 0 \\
11 & 0 & 0 & 0 & 0 & 5 & 0 & 128 & 0 & 0 \\
8 & 0 & 0 & 0 & 0 & 7 & 0 & 93 & 0 & 0 \\
7 & 0 & 0 & 0 & 0 & 0 & 0 & 99 & 0 & 0 \\
6 & 0 & 0 & 0 & 0 & 16 & 0 & 79 & 0 & 0 \\
5 & 4 & 0 & 0 & 0 & 8 & 28 & 8 & 0 & 0 \\
4 & 8 & 0 & 0 & 0 & 8 & 49 & 8 & 0 & 0 \\
3 & 5 & 0 & 0 & 0 & 5 & 34 & 0 & 0 & 0 \\
2 & 14 & 0 & 0 & 0 & 0 & 0 & 0 & 0 & 0 \\
1 & 47 & 0 & 0 & 0 & 0 & 0 & 5 & 0 & 5 \\
\hline
\end{tabular}


c219.std WCR-82H MOCNESS tow copepods

$4 i \cdot 2$

\begin{tabular}{rrrrrrrrrr} 
net\# & \#cund & \#ccal & \#fmin & \#mmin & \#cmin & \#fatt & \#fmon & \#felg & \#fcor \\
\hline 18 & 2448 & 326 & 471 & 200 & 1596 & 18 & 0 & 0 & 0 \\
17 & 195 & 409 & 506 & 214 & 1342 & 20 & 0 & 0 & 0 \\
16 & 0 & 1007 & 622 & 326 & 326 & 89 & 59 & 0 & 0 \\
15 & 0 & 154 & 200 & 77 & 46 & 15 & 0 & 0 & 0 \\
14 & 0 & 208 & 31 & 23 & 8 & 0 & 0 & 0 & 0 \\
13 & 0 & 85 & 7 & 0 & 0 & 0 & 0 & 0 & 7 \\
12 & 0 & 138 & 11 & 0 & 0 & 0 & 0 & 0 & 6 \\
11 & 0 & 29 & 0 & 0 & 0 & 0 & 5 & 0 & 0 \\
8 & 0 & 0 & 0 & 0 & 0 & 0 & 0 & 0 & 0 \\
7 & 0 & 0 & 0 & 0 & 7 & 0 & 0 & 0 & 14 \\
6 & 0 & 0 & 0 & 0 & 0 & 0 & 0 & 0 & 16 \\
5 & 0 & 0 & 4 & 0 & 4 & 0 & 0 & 0 & 57 \\
4 & 0 & 0 & 0 & 0 & 0 & 0 & 8 & 41 & 194 \\
3 & 0 & 0 & 0 & 0 & 0 & 10 & 5 & 92 & 233 \\
2 & 0 & 0 & 0 & 0 & 0 & 5 & 0 & 149 & 126 \\
1 & 0 & 0 & 0 & 0 & 5 & 14 & 0 & 57 & 33 \\
\hline
\end{tabular}

net\# \#mcor \#ccor \#fmey \#facr \#fprv \#farc \#furc \#mcla \#fssp

\begin{tabular}{rrrrrrrrrr}
\hline 18 & 0 & 0 & 0 & 508 & 18 & 961 & 18 & 54 & 0 \\
17 & 0 & 20 & 20 & 272 & 0 & 1712 & 0 & 156 & 0 \\
16 & 0 & 30 & 30 & 207 & 178 & 237 & 0 & 0 & 0 \\
15 & 0 & 0 & 0 & 0 & 0 & 0 & 31 & 46 & 0 \\
14 & 0 & 8 & 0 & 0 & 0 & 15 & 8 & 0 & 0 \\
13 & 0 & 7 & 0 & 0 & 0 & 0 & 0 & 0 & 0 \\
12 & 0 & 0 & 0 & 0 & 0 & 0 & 0 & 0 & 0 \\
11 & 5 & 10 & 0 & 0 & 0 & 0 & 0 & 0 & 0 \\
8 & 0 & 14 & 0 & 0 & 0 & 43 & 0 & 0 & 0 \\
7 & 7 & 35 & 0 & 0 & 0 & 141 & 0 & 0 & 7 \\
6 & 0 & 71 & 0 & 0 & 0 & 47 & 0 & 0 & 79 \\
5 & 12 & 226 & 0 & 0 & 0 & 65 & 4 & 0 & 719 \\
4 & 16 & 421 & 0 & 0 & 0 & 32 & 0 & 0 & 551 \\
3 & 49 & 379 & 0 & 0 & 0 & 5 & 5 & 0 & 306 \\
2 & 9 & 88 & 0 & 0 & 0 & 0 & 0 & 0 & 98 \\
1 & 5 & 43 & 0 & 0 & 0 & 0 & 5 & 0 & 146 \\
& & 0 & & & 0 & & & & \\
\hline
\end{tabular}


c219.std WCR-82H MOCNESS tow copepods

$4 i \cdot 3$

\begin{tabular}{|c|c|c|c|c|c|c|c|c|c|}
\hline net\# & \# fmim & \# fmty & \#faar & \#maar & \#fege & \# fgmi & \# feme & \#meme & \#fupl \\
\hline 18 & 0 & 0 & 0 & 0 & 0 & 0 & 0 & 0 & 0 \\
\hline 17 & 0 & 0 & 0 & 0 & 0 & 0 & 20 & 0 & 0 \\
\hline 16 & 0 & 0 & 0 & 0 & 741 & 0 & 0 & 0 & 0 \\
\hline 15 & 0 & 0 & 0 & 154 & 262 & 0 & 0 & 0 & 0 \\
\hline 14 & 0 & 0 & 0 & 69 & 8 & 0 & 0 & 0 & 0 \\
\hline 13 & 0 & 0 & 0 & 7 & 20 & 0 & 0 & 0 & 0 \\
\hline 12 & 0 & 0 & 0 & 17 & 17 & 0 & 0 & 0 & 0 \\
\hline 11 & 0 & 0 & 0 & 5 & 5 & 0 & 0 & 0 & 0 \\
\hline 8 & 0 & 0 & 0 & 0 & 14 & 0 & 0 & 0 & 0 \\
\hline 7 & 7 & 0 & 0 & 0 & 0 & 0 & 0 & 0 & 0 \\
\hline 6 & 39 & 0 & 0 & 0 & 0 & 87 & 0 & 0 & 0 \\
\hline 5 & 24 & 0 & 0 & 0 & 0 & 12 & 16 & 0 & 8 \\
\hline 4 & 32 & 0 & 8 & 0 & 0 & 16 & 89 & 16 & 105 \\
\hline 3 & 15 & 0 & 15 & 0 & 0 & 10 & 34 & 19 & 58 \\
\hline 2 & 5 & 9 & 5 & 0 & 0 & 0 & 33 & 5 & 19 \\
\hline 1 & 14 & 28 & 0 & 0 & 0 & 0 & 14 & 0 & 14 \\
\hline
\end{tabular}

net\# \#mupl \#fumj \#fchi \#fmed \#mmed \#fmar \#mmar \#finc \#minc

\begin{tabular}{|c|c|c|c|c|c|c|c|c|c|}
\hline 18 & 0 & 0 & 0 & 0 & 0 & 54 & 18 & 0 & 0 \\
\hline 17 & 0 & 0 & 0 & 0 & 0 & 39 & 0 & 0 & 0 \\
\hline 16 & 0 & 0 & 0 & 0 & 0 & 89 & 30 & 0 & 0 \\
\hline 15 & 0 & 0 & 0 & 15 & 0 & 0 & 0 & 0 & 0 \\
\hline 14 & 0 & 0 & 0 & 0 & 0 & 0 & 0 & 0 & 0 \\
\hline 13 & 0 & 0 & 0 & 0 & 0 & 0 & 0 & 0 & 0 \\
\hline 12 & 0 & 0 & 0 & 6 & 0 & 0 & 0 & 0 & 0 \\
\hline 11 & 0 & 0 & 0 & 0 & 0 & 0 & 0 & 0 & 0 \\
\hline 8 & 0 & 0 & 0 & 7 & 0 & 0 & 0 & 0 & 0 \\
\hline 7 & 0 & 0 & 0 & 7 & 0 & 0 & 0 & 0 & 0 \\
\hline 6 & 8 & 0 & 0 & 16 & 24 & 0 & 0 & 0 & 0 \\
\hline 5 & 4 & 0 & 0 & 109 & 61 & 0 & 0 & 0 & 0 \\
\hline 4 & 24 & 16 & 16 & 49 & 16 & 0 & 0 & 0 & 0 \\
\hline 3 & 24 & 5 & 24 & 0 & 0 & 0 & 0 & 5 & 19 \\
\hline 2 & 9 & 0 & 9 & 0 & 0 & 0 & 0 & 0 & 5 \\
\hline 1 & 0 & 0 & 0 & 0 & 0 & 0 & 0 & 0 & 0 \\
\hline
\end{tabular}


c219.std WCR-82:H MOCNESS tow copepods

$4 i \cdot 4$

net\# \#ceuk \#fpsp \#fslo \#fsam \#fscu \#fsma \#mscp \#fbra \#mbra

\begin{tabular}{|c|c|c|c|c|c|c|c|c|c|}
\hline 18 & 4170 & 0 & 0 & 18 & 0 & 0 & 0 & 0 & 0 \\
\hline 17 & 2413 & 0 & 0 & 0 & 0 & 0 & 0 & 0 & 0 \\
\hline 16 & 889 & 0 & 0 & 0 & 0 & 0 & 0 & 0 & 0 \\
\hline 15 & 2939 & 0 & 0 & 0 & 0 & 0 & 0 & 108 & 62 \\
\hline 14 & 1341 & 15 & 0 & 0 & 0 & 0 & 0 & 23 & 0 \\
\hline 13 & 701 & 0 & 0 & 0 & 0 & 0 & 0 & 33 & 0 \\
\hline 12 & 371 & 0 & 0 & 0 & 0 & 0 & 0 & 11 & 6 \\
\hline 11 & 216 & 15 & 0 & 5 & 0 & 0 & 5 & 10 & 0 \\
\hline 8 & 215 & 0 & 0 & 7 & 0 & 0 & 0 & 0 & 0 \\
\hline 7 & 289 & 0 & 0 & 21 & 0 & 0 & 0 & 28 & 0 \\
\hline 6 & 150 & 0 & 79 & 481 & 79 & 0 & 63 & 0 & 8 \\
\hline 5 & 65 & 0 & 521 & 408 & 69 & 0 & 36 & 0 & 8 \\
\hline 4 & 49 & 0 & 97 & 49 & 49 & 8 & 16 & 0 & 0 \\
\hline 3 & 102 & 0 & 44 & 0 & 73 & 0 & 238 & 0 & 0 \\
\hline 2 & 37 & 0 & 51 & 0 & 0 & 5 & 19 & 0 & 0 \\
\hline 1 & 66 & 0 & 5 & 0 & 0 & 0 & 0 & 0 & 0 \\
\hline
\end{tabular}

net\# \#fdan \#mdan \#fden \#fvit \#msca \#ftst \#ftmt \#fven \#mven

\begin{tabular}{|c|c|c|c|c|c|c|c|c|c|}
\hline 18 & 181 & 18 & 0 & 0 & 0 & 18 & 0 & 0 & 0 \\
\hline 17 & 78 & 0 & 0 & 0 & 0 & 39 & 0 & 0 & 0 \\
\hline 16 & 0 & 0 & 0 & 0 & 59 & 30 & 0 & 0 & 0 \\
\hline 15 & 0 & 0 & 0 & 0 & 46 & 0 & 0 & 0 & 0 \\
\hline 14 & 0 & 0 & 8 & 23 & 39 & 0 & 0 & 0 & 0 \\
\hline 13 & 0 & 0 & 0 & 13 & 20 & 0 & 0 & 0 & 0 \\
\hline 12 & 0 & 0 & 11 & 6 & 17 & 0 & 0 & 0 & 0 \\
\hline 11 & 0 & 0 & 10 & 10 & 39 & 0 & 0 & 0 & 0 \\
\hline 8 & 0 & 0 & 0 & 14 & 7 & 0 & 0 & 0 & 0 \\
\hline 7 & 0 & 0 & 7 & 7 & 14 & 0 & 0 & 0 & 0 \\
\hline 6 & 0 & 0 & 55 & 0 & 71 & 0 & 0 & 0 & 0 \\
\hline 5 & 0 & 0 & 24 & 0 & 49 & 0 & 0 & 4 & 4 \\
\hline 4 & 0 & 0 & 24 & 0 & 235 & 0 & 0 & 16 & 8 \\
\hline 3 & 0 & 0 & 0 & 0 & 83 & 0 & 5 & 180 & 141 \\
\hline 2 & 0 & 0 & 0 & 0 & 0 & 0 & 5 & 126 & 47 \\
\hline 1 & 0 & 0 & 0 & 0 & 19 & 0 & 5 & 85 & 9 \\
\hline
\end{tabular}


c219.std WCR-82H MOCNESS tow copepods

$4 i \cdot 5$

\begin{tabular}{rrrrrrrrrr} 
net\# & \#fbrv & \#mbrv & \#mlon & \#cmet & \#fgra & \#mgra & \#fpis & \#mpis & \#cpsm \\
\hline 18 & 0 & 0 & 0 & 0 & 0 & 0 & 0 & 0 & 0 \\
17 & 0 & 0 & 0 & 0 & 78 & 0 & 0 & 20 & 39 \\
16 & 0 & 0 & 0 & 0 & 0 & 0 & 0 & 0 & 0 \\
15 & 0 & 0 & 0 & 0 & 0 & 0 & 0 & 0 & 0 \\
14 & 0 & 8 & 0 & 0 & 8 & 15 & 15 & 0 & 31 \\
13 & 0 & 0 & 0 & 0 & 0 & 0 & 0 & 0 & 33 \\
12 & 0 & 0 & 0 & 0 & 0 & 0 & 0 & 0 & 89 \\
11 & 0 & 0 & 0 & 0 & 10 & 20 & 25 & 0 & 520 \\
8 & 0 & 0 & 0 & 0 & 865 & 272 & 458 & 215 & 1087 \\
7 & 0 & 0 & 0 & 0 & 473 & 78 & 275 & 78 & 261 \\
6 & 0 & 0 & 0 & 0 & 292 & 529 & 0 & 8 & 237 \\
5 & 0 & 0 & 0 & 12 & 210 & 323 & 36 & 16 & 113 \\
4 & 0 & 0 & 0 & 478 & 16 & 8 & 57 & 73 & 113 \\
3 & 170 & 19 & 5 & 651 & 0 & 0 & 0 & 0 & 0 \\
2 & 93 & 182 & 5 & 33 & 0 & 0 & 0 & 0 & 0 \\
1 & 71 & 5 & 5 & 80 & 0 & 0 & 0 & 0 & 0 \\
\hline
\end{tabular}

net\# \#fabd \#mabd \#fxip \#mxip \#cplg \#fvio \#mvio \#flcl \#mlcl

\begin{tabular}{rrrrrrrrrr}
\hline 18 & 18 & 0 & 0 & 0 & 18 & 18 & 36 & 0 & 0 \\
17 & 20 & 0 & 0 & 0 & 311 & 0 & 20 & 0 & 0 \\
16 & 0 & 0 & 0 & 0 & 148 & 30 & 0 & 0 & 0 \\
15 & 0 & 0 & 0 & 0 & 354 & 0 & 0 & 0 & 0 \\
14 & 0 & 8 & 0 & 8 & 901 & 0 & 0 & 0 & 0 \\
13 & 0 & 0 & 7 & 0 & 210 & 0 & 0 & 7 & 0 \\
12 & 0 & 0 & 0 & 0 & 116 & 0 & 0 & 55 & 22 \\
11 & 5 & 0 & 0 & 0 & 118 & 0 & 0 & 103 & 39 \\
8 & 7 & 7 & 7 & 0 & 486 & 0 & 0 & 100 & 7 \\
7 & 106 & 7 & 0 & 7 & 1404 & 0 & 0 & 318 & 318 \\
6 & 205 & 103 & 0 & 0 & 418 & 0 & 0 & 268 & 252 \\
5 & 129 & 53 & 493 & 93 & 182 & 0 & 4 & 0 & 0 \\
4 & 32 & 57 & 41 & 32 & 364 & 0 & 0 & 0 & 0 \\
3 & 15 & 5 & 24 & 15 & 258 & 0 & 0 & 0 & 0 \\
2 & 23 & 19 & 19 & 33 & 88 & 0 & 0 & 0 & 0 \\
1 & 0 & 24 & 5 & 0 & 28 & 0 & 0 & 0 & 0 \\
\hline
\end{tabular}


c219.std WCR-82H MOCNESS tow copepods

$4 i \cdot 6$

net\# \#flut \#mlut \#fpap \#mpap \#fspi \#mspi \#fvip \#mvip \#fhet

\begin{tabular}{rrrrrrrrrr}
\hline 18 & 127 & 36 & 127 & 91 & 0 & 0 & 0 & 0 & 0 \\
17 & 1051 & 681 & 292 & 409 & 0 & 0 & 0 & 0 & 0 \\
16 & 6430 & 3852 & 178 & 296 & 0 & 0 & 0 & 0 & 0 \\
15 & 3646 & 1939 & 77 & 108 & 0 & 0 & 0 & 0 & 0 \\
14 & 1248 & 416 & 54 & 39 & 0 & 0 & 0 & 0 & 0 \\
13 & 753 & 111 & 20 & 59 & 7 & 7 & 0 & 0 & 0 \\
12 & 791 & 44 & 33 & 50 & 0 & 0 & 0 & 0 & 0 \\
11 & 574 & 79 & 34 & 93 & 5 & 5 & 0 & 0 & 0 \\
8 & 536 & 143 & 29 & 50 & 0 & 0 & 0 & 0 & 0 \\
7 & 254 & 21 & 127 & 28 & 35 & 14 & 14 & 7 & 0 \\
6 & 134 & 0 & 197 & 39 & 0 & 16 & 24 & 24 & 32 \\
5 & 89 & 16 & 97 & 16 & 0 & 0 & 0 & 0 & 8 \\
4 & 16 & 8 & 24 & 0 & 24 & 0 & 24 & 8 & 8 \\
3 & 53 & 19 & 29 & 0 & 10 & 0 & 15 & 63 & 24 \\
2 & 19 & 47 & 5 & 0 & 0 & 0 & 28 & 0 & 56 \\
1 & 24 & 9 & 14 & 0 & 0 & 0 & 19 & 0 & 5 \\
\hline
\end{tabular}

net\# \#mhet \#chet \#fhal \#mhal \#chal \#forn \#faug \#fhel \#mhel

\begin{tabular}{rrrrrrrrrr}
\hline 18 & 0 & 0 & 0 & 0 & 0 & 0 & 0 & 0 & 0 \\
17 & 0 & 370 & 0 & 0 & 0 & 0 & 0 & 0 & 0 \\
16 & 0 & 1126 & 6074 & 267 & 444 & 30 & 148 & 0 & 0 \\
15 & 0 & 308 & 523 & 108 & 1723 & 31 & 0 & 0 & 0 \\
14 & 0 & 270 & 223 & 54 & 5986 & 8 & 54 & 0 & 0 \\
13 & 0 & 85 & 177 & 20 & 2593 & 0 & 52 & 0 & 0 \\
12 & 0 & 161 & 72 & 6 & 769 & 0 & 17 & 0 & 0 \\
11 & 0 & 304 & 29 & 0 & 981 & 0 & 0 & 0 & 0 \\
8 & 0 & 322 & 72 & 0 & 887 & 7 & 7 & 0 & 0 \\
7 & 0 & 261 & 494 & 7 & 169 & 7 & 148 & 0 & 0 \\
6 & 8 & 347 & 426 & 0 & 505 & 63 & 16 & 0 & 0 \\
5 & 0 & 81 & 194 & 0 & 145 & 69 & 28 & 32 & 24 \\
4 & 8 & 0 & 81 & 0 & 16 & 16 & 81 & 130 & 97 \\
3 & 34 & 102 & 15 & 0 & 0 & 0 & 204 & 83 & 24 \\
2 & 23 & 0 & 0 & 0 & 0 & 0 & 70 & 9 & 5 \\
1 & 5 & 28 & 0 & 0 & 5 & 0 & 19 & 9 & 9 \\
\hline
\end{tabular}


c219.std WCR-82H MOCNESS tow copepods $4 i-7$

net\# \#chel \#fket \#mket \#fclo \#mclo \#fpak \#mpak \#fpbi \#mpbi

\begin{tabular}{|c|c|c|c|c|c|c|c|c|c|}
\hline 18 & 0 & 0 & 0 & 0 & 0 & 0 & 36 & 0 & 91 \\
\hline 17 & 0 & 0 & 0 & 0 & 0 & 20 & 0 & 292 & 253 \\
\hline 16 & 0 & 0 & 0 & 0 & 0 & 0 & 0 & 356 & 178 \\
\hline 15 & 0 & 0 & 0 & 0 & 0 & 15 & 0 & 31 & 0 \\
\hline 14 & 0 & 0 & 0 & 0 & 0 & 0 & 0 & 8 & 0 \\
\hline 13 & 0 & 0 & 0 & 0 & 0 & 0 & 0 & 0 & 0 \\
\hline 12 & 0 & 0 & 0 & 0 & 0 & 0 & 0 & 0 & 6 \\
\hline 11 & 0 & 0 & 0 & 0 & 5 & 0 & 5 & 0 & 0 \\
\hline 8 & 0 & 0 & 0 & 0 & 0 & 0 & 0 & 0 & 0 \\
\hline 7 & 14 & 0 & 0 & 0 & 0 & 0 & 0 & 0 & 0 \\
\hline 6 & 134 & 32 & 8 & 0 & 0 & 0 & 0 & 8 & 0 \\
\hline 5 & 170 & 12 & 12 & 69 & 73 & 0 & 0 & 0 & 0 \\
\hline 4 & 97 & 0 & 0 & 0 & 0 & 8 & 0 & 0 & 0 \\
\hline 3 & 190 & 0 & 0 & 0 & 0 & 0 & 0 & 0 & 0 \\
\hline 2 & 5 & 0 & 0 & 0 & 0 & 0 & 0 & 0 & 0 \\
\hline 1 & 38 & 0 & 0 & 0 & 0 & 0 & 0 & 0 & 0 \\
\hline
\end{tabular}

net\# \#fpon \#fada \#fneg \#fmis \#mmis \#cmis \#oith \#harp \#totl

\begin{tabular}{|c|c|c|c|c|c|c|c|c|c|}
\hline 18 & 54 & 0 & 598 & 0 & 36 & 2937 & 218 & 5821 & 22303 \\
\hline 17 & 20 & 0 & 175 & 58 & 0 & 4300 & 292 & 4786 & 21751 \\
\hline 16 & 59 & 0 & 296 & 800 & 119 & 4682 & 4474 & 4356 & 41689 \\
\hline 15 & 0 & 0 & 0 & 92 & 0 & 1708 & 2446 & 2877 & 22046 \\
\hline 14 & 0 & 0 & 0 & 46 & 0 & 1325 & 370 & 1587 & 14769 \\
\hline 13 & 0 & 0 & 20 & 0 & 20 & 327 & 20 & 832 & 6392 \\
\hline 12 & 0 & 6 & 6 & 17 & 6 & 343 & 33 & 487 & 3879 \\
\hline 11 & 0 & 0 & 10 & 10 & 15 & 442 & 157 & 314 & 4401 \\
\hline 8 & 0 & 0 & 0 & 7 & 22 & 672 & 43 & 200 & 6930 \\
\hline 7 & 0 & 0 & 0 & 64 & 0 & 649 & 198 & 92 & 6633 \\
\hline 6 & 0 & 0 & 0 & 39 & 39 & 3258 & 166 & 142 & 9450 \\
\hline 5 & 0 & 4 & 0 & 40 & 8 & 1866 & 28 & 267 & 7585 \\
\hline 4 & 0 & 0 & 0 & 73 & 16 & 1757 & 24 & 648 & 6736 \\
\hline 3 & 0 & 0 & 0 & 63 & 73 & 1540 & 10 & 170 & 6740 \\
\hline 2 & 0 & 0 & 0 & 56 & 42 & 1275 & 9 & 144 & 4584 \\
\hline 1 & 0 & 0 & 5 & 109 & 14 & 1019 & 0 & 118 & 3832 \\
\hline
\end{tabular}


c219.int WCR-82H MOCNESS tow copepods

$4 j-1$

\begin{tabular}{rrrrrrrrrr} 
net\# & midd & depi & volf & aliq & iffin & imfin & ivfin & icfin & ifhyp \\
\hline 18 & 13 & 25 & 221 & 4 & 0 & 0 & 0 & 0 & 0 \\
17 & 38 & 25 & 206 & 4 & 0 & 0 & 0 & 0 & 0 \\
16 & 63 & 25 & 135 & 4 & 0 & 0 & 0 & 0 & 0 \\
15 & 88 & 25 & 130 & 2 & 0 & 0 & 0 & 0 & 0 \\
14 & 113 & 25 & 130 & 1 & 0 & 0 & 0 & 0 & 0 \\
13 & 138 & 25 & 153 & 1 & 0 & 0 & 0 & 0 & 0 \\
12 & 163 & 25 & 181 & 1 & 0 & 0 & 0 & 0 & 0 \\
11 & 188 & 25 & 204 & 1 & 0 & 0 & 0 & 0 & 0 \\
8 & 250 & 100 & 559 & 4 & 0 & 0 & 0 & 0 & 0 \\
7 & 350 & 100 & 567 & 4 & 0 & 0 & 1 & 0 & 0 \\
6 & 450 & 100 & 507 & 4 & 0 & 0 & 2 & 1 & 0 \\
5 & 550 & 100 & 495 & 2 & 0 & 0 & 2 & 1 & 0 \\
4 & 650 & 100 & 494 & 4 & 0 & 0 & 4 & 2 & 0 \\
3 & 750 & 100 & 823 & 4 & 2 & 0 & 49 & 19 & 0 \\
2 & 850 & 100 & 430 & 2 & 4 & 0 & 154 & 51 & 1 \\
1 & 950 & 100 & 424 & 2 & 7 & 0 & 259 & 98 & 2 \\
\hline
\end{tabular}

\begin{tabular}{crrrrrrrrr} 
net\# & ichyp & iften & imten & icten & ifngr & imngr & icngr & ifund imund \\
\hline 18 & 0 & 1 & 0 & 1 & 0 & 0 & 3 & 7 & 11 \\
17 & 0 & 7 & 0 & 4 & 1 & 0 & 12 & 8 & 13 \\
16 & 0 & 28 & 5 & 33 & 1 & 0 & 16 & 8 & 13 \\
15 & 0 & 39 & 16 & 58 & 1 & 0 & 16 & 8 & 13 \\
14 & 0 & 42 & 18 & 59 & 1 & 0 & 16 & 8 & 13 \\
13 & 0 & 43 & 20 & 59 & 1 & 0 & 17 & 8 & 13 \\
12 & 0 & 43 & 21 & 60 & 1 & 0 & 19 & 8 & 13 \\
11 & 0 & 43 & 21 & 60 & 1 & 0 & 22 & 8 & 13 \\
8 & 0 & 43 & 21 & 60 & 2 & 0 & 31 & 8 & 13 \\
7 & 0 & 43 & 21 & 60 & 2 & 0 & 41 & 8 & 13 \\
6 & 0 & 43 & 21 & 60 & 4 & 0 & 49 & 8 & 13 \\
5 & 0 & 43 & 21 & 60 & 5 & 3 & 50 & 8 & 13 \\
4 & 1 & 43 & 21 & 60 & 5 & 8 & 51 & 8 & 13 \\
3 & 2 & 43 & 21 & 60 & 6 & 11 & 51 & 8 & 13 \\
2 & 3 & 43 & 21 & 60 & 6 & 11 & 51 & 8 & 13 \\
1 & 8 & 43 & 21 & 60 & 6 & 11 & 51 & 8 & 13 \\
\hline
\end{tabular}


c219.int WCR-82H MOCNESS tow copepods

$4 j-2$

net\# icund iccal ifmin immin icmin ifatt ifmon ifelg ifcor

\begin{tabular}{rrrrrrrrrr}
\hline 18 & 61 & 8 & 12 & 5 & 40 & 0 & 0 & 0 & 0 \\
17 & 66 & 18 & 24 & 10 & 73 & 1 & 0 & 0 & 0 \\
16 & 66 & 43 & 40 & 18 & 82 & 3 & 1 & 0 & 0 \\
15 & 66 & 47 & 45 & 20 & 83 & 4 & 1 & 0 & 0 \\
14 & 66 & 53 & 46 & 21 & 83 & 4 & 1 & 0 & 0 \\
13 & 66 & 55 & 46 & 21 & 83 & 4 & 1 & 0 & 0 \\
12 & 66 & 58 & 46 & 21 & 83 & 4 & 1 & 0 & 0 \\
11 & 66 & 59 & 46 & 21 & 83 & 4 & 2 & 0 & 0 \\
8 & 66 & 59 & 46 & 21 & 83 & 4 & 2 & 0 & 0 \\
7 & 66 & 59 & 46 & 21 & 84 & 4 & 2 & 0 & 2 \\
6 & 66 & 59 & 46 & 21 & 84 & 4 & 2 & 0 & 3 \\
5 & 66 & 59 & 47 & 21 & 84 & 4 & 2 & 0 & 9 \\
4 & 66 & 59 & 47 & 21 & 84 & 4 & 2 & 4 & 28 \\
3 & 66 & 59 & 47 & 21 & 84 & 5 & 3 & 13 & 52 \\
2 & 66 & 59 & 47 & 21 & 84 & 5 & 3 & 28 & 64 \\
1 & 66 & 59 & 47 & 21 & 85 & 6 & 3 & 34 & 68 \\
\hline
\end{tabular}

\begin{tabular}{rrrrrrrrrr} 
net\# & imcor & iccor & ifmey & ifacr & ifprv & ifarc & ifurc & imcla & ifss? \\
\hline 18 & 0 & 0 & 0 & 13 & 0 & 24 & 0 & 1 & 0 \\
17 & 0 & 1 & 0 & 20 & 0 & 67 & 0 & 5 & 0 \\
16 & 0 & 1 & 1 & 25 & 5 & 73 & 0 & 5 & 0 \\
15 & 0 & 1 & 1 & 25 & 5 & 73 & 1 & 6 & 0 \\
14 & 0 & 1 & 1 & 25 & 5 & 73 & 1 & 6 & 0 \\
13 & 0 & 2 & 1 & 25 & 5 & 73 & 1 & 6 & 0 \\
12 & 0 & 2 & 1 & 25 & 5 & 73 & 1 & 6 & 0 \\
11 & 0 & 2 & 1 & 25 & 5 & 73 & 1 & 6 & 0 \\
8 & 0 & 3 & 1 & 25 & 5 & 78 & 1 & 6 & 0 \\
7 & 1 & 7 & 1 & 25 & 5 & 92 & 1 & 6 & 1 \\
6 & 1 & 14 & 1 & 25 & 5 & 96 & 1 & 6 & 9 \\
5 & 2 & 37 & 1 & 25 & 5 & 103 & 2 & 6 & 80 \\
4 & 4 & 79 & 1 & 25 & 5 & 106 & 2 & 6 & 135 \\
3 & 9 & 117 & 1 & 25 & 5 & 107 & 2 & 6 & 166 \\
2 & 9 & 125 & 1 & 25 & 5 & 107 & 2 & 6 & 176 \\
1 & 10 & 130 & 1 & 25 & 5 & 107 & 3 & 6 & 191 \\
\hline
\end{tabular}


c219.int WCR-82H MOCNESS tow copepods

$4 j-3$

\begin{tabular}{rrrrrrrrrr} 
net\# & ifmim & ifmty & ifaar & imaar & ifege & ifgmi & ifeme & imeme & ifupl \\
\hline 18 & 0 & 0 & 0 & 0 & 0 & 0 & 0 & 0 & 0 \\
17 & 0 & 0 & 0 & 0 & 0 & 0 & 0 & 0 & 0 \\
16 & 0 & 0 & 0 & 0 & 19 & 0 & 0 & 0 & 0 \\
15 & 0 & 0 & 0 & 4 & 25 & 0 & 0 & 0 & 0 \\
14 & 0 & 0 & 0 & 6 & 25 & 0 & 0 & 0 & 0 \\
13 & 0 & 0 & 0 & 6 & 26 & 0 & 0 & 0 & 0 \\
12 & 0 & 0 & 0 & 6 & 26 & 0 & 0 & 0 & 0 \\
11 & 0 & 0 & 0 & 6 & 26 & 0 & 0 & 0 & 0 \\
8 & 0 & 0 & 0 & 6 & 28 & 0 & 0 & 0 & 0 \\
7 & 1 & 0 & 0 & 6 & 28 & 0 & 0 & 0 & 0 \\
6 & 5 & 0 & 0 & 6 & 28 & 9 & 0 & 0 & 0 \\
5 & 7 & 0 & 0 & 6 & 28 & 10 & 2 & 0 & 1 \\
4 & 10 & 0 & 1 & 6 & 28 & 12 & 11 & 2 & 11 \\
3 & 12 & 0 & 2 & 6 & 28 & 12 & 14 & 4 & 17 \\
2 & 12 & 1 & 3 & 6 & 28 & 12 & 18 & 4 & 19 \\
1 & 14 & 4 & 3 & 6 & 28 & 12 & 19 & 4 & 20 \\
\hline
\end{tabular}

\begin{tabular}{crrrrrrrrr} 
net\# & imupl & ifumj & ifchi & ifmed & immed & ifmar & immar & ifinc & iminc \\
\hline 18 & 0 & 0 & 0 & 0 & 0 & 1 & 0 & 0 & 0 \\
17 & 0 & 0 & 0 & 0 & 0 & 2 & 0 & 0 & 0 \\
16 & 0 & 0 & 0 & 0 & 0 & 5 & 1 & 0 & 0 \\
15 & 0 & 0 & 0 & 0 & 0 & 5 & 1 & 0 & 0 \\
14 & 0 & 0 & 0 & 0 & 0 & 5 & 1 & 0 & 0 \\
13 & 0 & 0 & 0 & 0 & 0 & 5 & 1 & 0 & 0 \\
12 & 0 & 0 & 0 & 1 & 0 & 5 & 1 & 0 & 0 \\
11 & 0 & 0 & 0 & 1 & 0 & 5 & 1 & 0 & 0 \\
8 & 0 & 0 & 0 & 1 & 0 & 5 & 1 & 0 & 0 \\
7 & 0 & 0 & 0 & 2 & 0 & 5 & 1 & 0 & 0 \\
6 & 1 & 0 & 0 & 4 & 2 & 5 & 1 & 0 & 0 \\
5 & 1 & 0 & 0 & 14 & 8 & 5 & 1 & 0 & 0 \\
4 & 4 & 2 & 2 & 19 & 10 & 5 & 1 & 0 & 0 \\
3 & 6 & 2 & 4 & 19 & 10 & 5 & 1 & 0 & 2 \\
2 & 7 & 2 & 5 & 19 & 10 & 5 & 1 & 0 & 2 \\
1 & 7 & 2 & 5 & 19 & 10 & 5 & 1 & 0 & 2 \\
\hline
\end{tabular}


c219.int WCR-82H MOCNESS tow copepods

$4 j-4$

\begin{tabular}{cccrrrrrrr} 
net\# & iceuk & ifpsp & ifslo & ifsam & ifscu & ifsma & imscp & ifbra & imbra \\
\hline 18 & 104 & 0 & 0 & 0 & 0 & 0 & 0 & 0 & 0 \\
17 & 165 & 0 & 0 & 0 & 0 & 0 & 0 & 0 & 0 \\
16 & 187 & 0 & 0 & 0 & 0 & 0 & 0 & 0 & 0 \\
15 & 261 & 0 & 0 & 0 & 0 & 0 & 0 & 3 & 2 \\
14 & 294 & 0 & 0 & 0 & 0 & 0 & 0 & 3 & 2 \\
13 & 311 & 0 & 0 & 0 & 0 & 0 & 0 & 4 & 2 \\
12 & 321 & 0 & 0 & 0 & 0 & 0 & 0 & 4 & 2 \\
11 & 326 & 1 & 0 & 1 & 0 & 0 & 0 & 5 & 2 \\
8 & 348 & 1 & 0 & 1 & 0 & 0 & 0 & 5 & 2 \\
7 & 377 & 1 & 0 & 3 & 0 & 0 & 0 & 7 & 2 \\
6 & 391 & 1 & 8 & 52 & 8 & 0 & 6 & 7 & 2 \\
5 & 398 & 1 & 60 & 92 & 15 & 0 & 10 & 7 & 3 \\
4 & 402 & 1 & 70 & 97 & 20 & 1 & 12 & 7 & 3 \\
3 & 413 & 1 & 74 & 97 & 27 & 1 & 35 & 7 & 3 \\
2 & 416 & 1 & 79 & 97 & 27 & 1 & 37 & 7 & 3 \\
1 & 423 & 1 & 80 & 97 & 27 & 1 & 37 & 7 & 3 \\
\hline
\end{tabular}

\begin{tabular}{crrrrrrrrr} 
net\# & ifdan & imdan & ifden & ifvit & imsca & iftst & iftmt & ifven & imven \\
\hline 18 & 5 & 0 & 0 & 0 & 0 & 0 & 0 & 0 & 0 \\
17 & 6 & 0 & 0 & 0 & 0 & 1 & 0 & 0 & 0 \\
16 & 6 & 0 & 0 & 0 & 1 & 2 & 0 & 0 & 0 \\
15 & 6 & 0 & 0 & 0 & 3 & 2 & 0 & 0 & 0 \\
14 & 6 & 0 & 0 & 1 & 4 & 2 & 0 & 0 & 0 \\
13 & 6 & 0 & 0 & 1 & 4 & 2 & 0 & 0 & 0 \\
12 & 6 & 0 & 0 & 1 & 5 & 2 & 0 & 0 & 0 \\
11 & 6 & 0 & 1 & 1 & 5 & 2 & 0 & 0 & 0 \\
8 & 6 & 0 & 1 & 3 & 6 & 2 & 0 & 0 & 0 \\
7 & 6 & 0 & 1 & 3 & 8 & 2 & 0 & 0 & 0 \\
6 & 6 & 0 & 7 & 3 & 15 & 2 & 0 & 0 & 0 \\
5 & 6 & 0 & 9 & 3 & 20 & 2 & 0 & 0 & 0 \\
4 & 6 & 0 & 12 & 3 & 43 & 2 & 0 & 2 & 1 \\
3 & 6 & 0 & 12 & 3 & 51 & 2 & 0 & 20 & 15 \\
2 & 6 & 0 & 12 & 3 & 51 & 2 & 1 & 33 & 20 \\
1 & 6 & 0 & 12 & 3 & 53 & 2 & 1 & 41 & 21 \\
\hline
\end{tabular}


c219.int WCR-82H MOCNESS tow copepods

$4 j-5$

\begin{tabular}{rrrrrrrrrr} 
net\# & ifbrv & imbrv & imlon & icmet & ifgra & imgra & ifpis & impis & icpsm \\
\hline 18 & 0 & 0 & 0 & 0 & 0 & 0 & 0 & 0 & 0 \\
17 & 0 & 0 & 0 & 0 & 2 & 0 & 0 & 0 & 1 \\
16 & 0 & 0 & 0 & 0 & 2 & 0 & 0 & 0 & 1 \\
15 & 0 & 0 & 0 & 0 & 2 & 0 & 0 & 0 & 1 \\
14 & 0 & 0 & 0 & 0 & 2 & 0 & 0 & 0 & 2 \\
13 & 0 & 0 & 0 & 0 & 2 & 0 & 0 & 0 & 3 \\
12 & 0 & 0 & 0 & 0 & 2 & 0 & 0 & 0 & 5 \\
11 & 0 & 0 & 0 & 0 & 2 & 1 & 1 & 0 & 18 \\
8 & 0 & 0 & 0 & 0 & 89 & 28 & 47 & 22 & 126 \\
7 & 0 & 0 & 0 & 0 & 136 & 36 & 74 & 30 & 153 \\
6 & 0 & 0 & 0 & 0 & 165 & 89 & 74 & 31 & 176 \\
5 & 0 & 0 & 0 & 1 & 186 & 121 & 78 & 32 & 188 \\
4 & 0 & 0 & 0 & 49 & 188 & 122 & 84 & 39 & 199 \\
3 & 17 & 2 & 0 & 114 & 188 & 122 & 84 & 39 & 199 \\
2 & 26 & 20 & 1 & 117 & 188 & 122 & 84 & 39 & 199 \\
1 & 33 & 21 & 1 & 125 & 188 & 122 & 84 & 39 & 199 \\
\hline
\end{tabular}

net\# ifabd imabd ifxip imxip icplg ifvio imvio iflcl imlcl

\begin{tabular}{rrrrrrrrrr}
\hline 18 & 0 & 0 & 0 & 0 & 0 & 0 & 1 & 0 & 0 \\
17 & 1 & 0 & 0 & 0 & 8 & 0 & 1 & 0 & 0 \\
16 & 1 & 0 & 0 & 0 & 12 & 1 & 1 & 0 & 0 \\
15 & 1 & 0 & 0 & 0 & 21 & 1 & 1 & 0 & 0 \\
14 & 1 & 0 & 0 & 0 & 43 & 1 & 1 & 0 & 0 \\
13 & 1 & 0 & 0 & 0 & 49 & 1 & 1 & 0 & 0 \\
12 & 1 & 0 & 0 & 0 & 52 & 1 & 1 & 2 & 1 \\
11 & 1 & 0 & 0 & 0 & 55 & 1 & 1 & 4 & 2 \\
8 & 2 & 1 & 1 & 0 & 103 & 1 & 1 & 14 & 2 \\
7 & 12 & 2 & 1 & 1 & 244 & 1 & 1 & 46 & 34 \\
6 & 33 & 12 & 1 & 1 & 286 & 1 & 1 & 73 & 59 \\
5 & 46 & 17 & 50 & 10 & 304 & 1 & 2 & 73 & 59 \\
4 & 49 & 23 & 54 & 13 & 340 & 1 & 2 & 73 & 59 \\
3 & 50 & 23 & 57 & 15 & 366 & 1 & 2 & 73 & 59 \\
2 & 53 & 25 & 59 & 18 & 375 & 1 & 2 & 73 & 59 \\
1 & 53 & 28 & 59 & 18 & 378 & 1 & 2 & 73 & 59 \\
\hline
\end{tabular}


c219.int WCR-82H MOCNESS tow copepods

$4 i-6$

net\# iflut imlut ifpap impap ifspi imspi ifvip imvip ifhet

\begin{tabular}{rrrrrrrrrr}
\hline 18 & 3 & 1 & 3 & 2 & 0 & 0 & 0 & 0 & 0 \\
17 & 30 & 18 & 11 & 12 & 0 & 0 & 0 & 0 & 0 \\
16 & 190 & 114 & 15 & 20 & 0 & 0 & 0 & 0 & 0 \\
15 & 282 & 163 & 17 & 23 & 0 & 0 & 0 & 0 & 0 \\
14 & 313 & 173 & 18 & 24 & 0 & 0 & 0 & 0 & 0 \\
13 & 331 & 176 & 19 & 25 & 0 & 0 & 0 & 0 & 0 \\
12 & 351 & 177 & 20 & 26 & 0 & 0 & 0 & 0 & 0 \\
11 & 365 & 179 & 20 & 29 & 0 & 0 & 0 & 0 & 0 \\
8 & 419 & 193 & 23 & 34 & 0 & 0 & 0 & 0 & 0 \\
7 & 444 & 195 & 36 & 36 & 4 & 2 & 1 & 1 & 0 \\
6 & 457 & 195 & 56 & 40 & 4 & 3 & 4 & 3 & 3 \\
5 & 467 & 197 & 65 & 42 & 4 & 3 & 4 & 3 & 4 \\
4 & 468 & 198 & 68 & 42 & 6 & 3 & 6 & 4 & 5 \\
3 & 473 & 200 & 71 & 42 & 7 & 3 & 8 & 10 & 7 \\
2 & 475 & 205 & 71 & 42 & 7 & 3 & 10 & 10 & 13 \\
1 & 478 & 206 & 73 & 42 & 7 & 3 & 12 & 10 & 13 \\
\hline
\end{tabular}

net\# imhet ichet ifhal imhal ichal iforn ifaug ifhel imhel

\begin{tabular}{rrrrrrrrrr}
\hline 18 & 0 & 0 & 0 & 0 & 0 & 0 & 0 & 0 & 0 \\
17 & 0 & 9 & 0 & 0 & 0 & 0 & 0 & 0 & 0 \\
16 & 0 & 37 & 152 & 7 & 11 & 1 & 4 & 0 & 0 \\
15 & 0 & 45 & 165 & 9 & 54 & 2 & 4 & 0 & 0 \\
14 & 0 & 52 & 171 & 11 & 204 & 2 & 5 & 0 & 0 \\
13 & 0 & 54 & 175 & 11 & 269 & 2 & 6 & 0 & 0 \\
12 & 0 & 58 & 177 & 11 & 288 & 2 & 7 & 0 & 0 \\
11 & 0 & 66 & 177 & 11 & 313 & 2 & 7 & 0 & 0 \\
8 & 0 & 98 & 184 & 11 & 402 & 2 & 7 & 0 & 0 \\
7 & 0 & 124 & 234 & 12 & 419 & 3 & 22 & 0 & 0 \\
6 & 1 & 158 & 276 & 12 & 469 & 9 & 24 & 0 & 0 \\
5 & 1 & 167 & 295 & 12 & 484 & 16 & 27 & 3 & 2 \\
4 & 2 & 167 & 304 & 12 & 485 & 18 & 35 & 16 & 12 \\
3 & 5 & 177 & 305 & 12 & 485 & 18 & 55 & 24 & 15 \\
2 & 177 & 305 & 12 & 485 & 18 & 62 & 25 & 15 \\
1 & 8 & 180 & 305 & 12 & 486 & 18 & 64 & 26 & 16 \\
\hline
\end{tabular}


C219.int WCR-82H MOCNESS tow copepods

$4 j-7$

net\# ichel ifket imket ifclo imclo ifpak impak ifpbi impbi

\begin{tabular}{rrlllllllr}
\hline 18 & 0 & 0 & 0 & 0 & 0 & 0 & 1 & 0 & 2 \\
17 & 0 & 0 & 0 & 0 & 0 & 0 & 1 & 7 & 9 \\
16 & 0 & 0 & 0 & 0 & 0 & 0 & 1 & 16 & 13 \\
15 & 0 & 0 & 0 & 0 & 0 & 1 & 1 & 17 & 13 \\
14 & 0 & 0 & 0 & 0 & 0 & 1 & 1 & 17 & 13 \\
13 & 0 & 0 & 0 & 0 & 0 & 1 & 1 & 17 & 13 \\
12 & 0 & 0 & 0 & 0 & 0 & 1 & 1 & 17 & 13 \\
11 & 0 & 0 & 0 & 0 & 0 & 1 & 1 & 17 & 13 \\
8 & 0 & 0 & 0 & 0 & 0 & 1 & 1 & 17 & 13 \\
7 & 1 & 0 & 0 & 0 & 0 & 1 & 1 & 17 & 13 \\
6 & 15 & 3 & 1 & 0 & 0 & 1 & 1 & 18 & 13 \\
5 & 32 & 4 & 2 & 7 & 7 & 1 & 1 & 18 & 13 \\
4 & 41 & 4 & 2 & 7 & 7 & 2 & 1 & 18 & 13 \\
3 & 60 & 4 & 2 & 7 & 7 & 2 & 1 & 18 & 13 \\
2 & 61 & 4 & 2 & 7 & 7 & 2 & 1 & 18 & 13 \\
1 & 65 & 4 & 2 & 7 & 7 & 2 & 1 & 18 & 13 \\
\hline
\end{tabular}

\begin{tabular}{|c|c|c|c|c|c|c|c|c|c|}
\hline net\# & ifpon & ifada & ifneg & ifmis & immis & icmis & ioith & iharp & itotl \\
\hline $\begin{array}{l}18 \\
17 \\
16 \\
15 \\
14 \\
13 \\
12 \\
11 \\
8 \\
7 \\
6 \\
5 \\
4 \\
3 \\
2 \\
1\end{array}$ & $\begin{array}{l}1 \\
2 \\
3 \\
3 \\
3 \\
3 \\
3 \\
3 \\
3 \\
3 \\
3 \\
3 \\
3 \\
3 \\
3 \\
3\end{array}$ & $\begin{array}{l}0 \\
0 \\
0 \\
0 \\
0 \\
0 \\
0 \\
0 \\
0 \\
0 \\
0 \\
1 \\
1 \\
1 \\
1 \\
1\end{array}$ & $\begin{array}{l}15 \\
19 \\
27 \\
27 \\
27 \\
27 \\
27 \\
28 \\
28 \\
28 \\
28 \\
28 \\
28 \\
28 \\
28 \\
28\end{array}$ & $\begin{array}{r}0 \\
1 \\
21 \\
24 \\
25 \\
25 \\
25 \\
26 \\
26 \\
33 \\
37 \\
41 \\
48 \\
54 \\
60 \\
71\end{array}$ & $\begin{array}{r}1 \\
1 \\
4 \\
4 \\
4 \\
4 \\
5 \\
5 \\
7 \\
7 \\
11 \\
12 \\
13 \\
21 \\
25 \\
26\end{array}$ & $\begin{array}{r}74 \\
181 \\
299 \\
342 \\
376 \\
384 \\
392 \\
403 \\
470 \\
535 \\
860 \\
1047 \\
1222 \\
1376 \\
1502 \\
1604\end{array}$ & $\begin{array}{r}5 \\
13 \\
124 \\
186 \\
195 \\
195 \\
196 \\
200 \\
204 \\
224 \\
241 \\
244 \\
246 \\
247 \\
248 \\
248\end{array}$ & $\begin{array}{l}146 \\
266 \\
375 \\
447 \\
486 \\
507 \\
519 \\
527 \\
547 \\
556 \\
570 \\
597 \\
662 \\
678 \\
693 \\
705\end{array}$ & $\begin{array}{r}555 \\
1102 \\
2144 \\
2699 \\
3073 \\
3229 \\
3324 \\
3437 \\
4131 \\
4791 \\
5737 \\
6492 \\
7169 \\
7846 \\
8306 \\
8688\end{array}$ \\
\hline
\end{tabular}


C219. cum WCR-82H MOCNESS tow copepods

$4 k-1$

\begin{tabular}{rrrrrrrrrr} 
net\# & midd & depi & volf & aliq & offin & omfin & ovfin & ocfin & sfhyp \\
\hline 18 & 13 & 25 & 221 & 4 & 0 & 0 & 0 & 0 & 0 \\
17 & 38 & 25 & 206 & 4 & 0 & 0 & 0 & 0 & 0 \\
16 & 63 & 25 & 135 & 4 & 0 & 0 & 0 & 0 & 0 \\
15 & 88 & 25 & 130 & 2 & 0 & 0 & 0 & 0 & 0 \\
14 & 113 & 25 & 130 & 1 & 0 & 0 & 0 & 0 & 0 \\
13 & 138 & 25 & 153 & 1 & 0 & 0 & 0 & 0 & 0 \\
12 & 163 & 25 & 181 & 1 & 0 & 0 & 0 & 0 & 0 \\
11 & 188 & 25 & 204 & 1 & 0 & 0 & 0 & 0 & 0 \\
8 & 250 & 100 & 559 & 4 & 0 & 0 & 0 & 0 & 0 \\
7 & 350 & 100 & 567 & 4 & 0 & 0 & 0 & 0 & 0 \\
6 & 450 & 100 & 507 & 4 & 0 & 0 & 1 & 1 & 0 \\
5 & 550 & 100 & 495 & 2 & 0 & 0 & 1 & 1 & 0 \\
4 & 650 & 100 & 494 & 4 & 0 & 0 & 2 & 2 & 0 \\
3 & 750 & 100 & 823 & 4 & 29 & 0 & 19 & 20 & 0 \\
2 & 850 & 100 & 430 & 2 & 64 & 100 & 60 & 52 & 60 \\
1 & 950 & 100 & 424 & 2 & 100 & 100 & 100 & 100 & 100 \\
\hline
\end{tabular}

net\# schyp \&ften smten often ofngr osmngr scngr sfund omund.

\begin{tabular}{rrrrrrrrrr}
\hline 18 & 0 & 2 & 0 & 2 & 0 & 0 & 6 & 86 & 85 \\
17 & 0 & 16 & 0 & 6 & 17 & 0 & 23 & 91 & 96 \\
16 & 0 & 65 & 25 & 55 & 17 & 0 & 31 & 100 & 96 \\
15 & 0 & 89 & 77 & 98 & 23 & 0 & 31 & 100 & 96 \\
14 & 0 & 97 & 86 & 99 & 23 & 0 & 32 & 100 & 96 \\
13 & 0 & 100 & 95 & 100 & 23 & 0 & 33 & 100 & 96 \\
12 & 0 & 100 & 100 & 100 & 23 & 0 & 37 & 100 & 96 \\
11 & 0 & 100 & 100 & 100 & 25 & 0 & 43 & 100 & 96 \\
8 & 0 & 100 & 100 & 100 & 37 & 0 & 61 & 100 & 96 \\
7 & 0 & 100 & 100 & 100 & 37 & 0 & 80 & 100 & 96 \\
6 & 0 & 100 & 100 & 100 & 64 & 0 & 96 & 100 & 96 \\
5 & 5 & 100 & 100 & 100 & 78 & 26 & 97 & 100 & 96 \\
4 & 15 & 100 & 100 & 100 & 92 & 69 & 99 & 100 & 96 \\
3 & 22 & 100 & 100 & 100 & 100 & 100 & 99 & 100 & 96 \\
2 & 40 & 100 & 100 & 100 & 100 & 100 & 99 & 100 & 96 \\
1 & 100 & 100 & 100 & 100 & 100 & 100 & 100 & 100 & 100 \\
\hline
\end{tabular}


c219. cum WCR-82H MOCNESS tow copepods

$4 k-2$

net\# \%cund occal sfmin ommin ocmin sfatt ofmon sfelg ofcor

\begin{tabular}{rrrrrrrrrr}
\hline 18 & 93 & 14 & 25 & 24 & 47 & 7 & 0 & 0 & 0 \\
17 & 100 & 31 & 52 & 49 & 87 & 15 & 0 & 0 & 0 \\
16 & 100 & 74 & 86 & 88 & 97 & 49 & 51 & 0 & 0 \\
15 & 100 & 80 & 97 & 97 & 98 & 55 & 51 & 0 & 0 \\
14 & 100 & 89 & 98 & 100 & 98 & 55 & 51 & 0 & 0 \\
13 & 100 & 93 & 99 & 100 & 98 & 55 & 51 & 0 & 0 \\
12 & 100 & 99 & 99 & 100 & 98 & 55 & 51 & 0 & 0 \\
11 & 100 & 100 & 99 & 100 & 98 & 55 & 55 & 0 & 0 \\
8 & 100 & 100 & 99 & 100 & 98 & 55 & 55 & 0 & 0 \\
7 & 100 & 100 & 99 & 100 & 99 & 55 & 55 & 0 & 3 \\
6 & 100 & 100 & 99 & 100 & 99 & 55 & 55 & 0 & 5 \\
5 & 100 & 100 & 100 & 100 & 99 & 55 & 55 & 0 & 13 \\
4 & 100 & 100 & 100 & 100 & 99 & 55 & 83 & 12 & 42 \\
3 & 100 & 100 & 100 & 100 & 99 & 71 & 100 & 39 & 77 \\
2 & 100 & 100 & 100 & 100 & 99 & 78 & 100 & 83 & 95 \\
1 & 100 & 100 & 100 & 100 & 100 & 100 & 100 & 100 & 100 \\
\hline
\end{tabular}

net\# \%mcor \%ccor \%fmey \%facr \%fprv \%farc \%furc \%mcla $\frac{\circ}{\partial}$ ssp

\begin{tabular}{rrrrrrrrrr}
\hline 18 & 0 & 0 & 0 & 51 & 9 & 23 & 16 & 21 & 0 \\
17 & 0 & 0 & 40 & 79 & 9 & 63 & 16 & 82 & 0 \\
16 & 0 & 1 & 100 & 100 & 100 & 68 & 16 & 82 & 0 \\
15 & 0 & 1 & 100 & 100 & 100 & 68 & 44 & 100 & 0 \\
14 & 0 & 1 & 100 & 100 & 100 & 69 & 51 & 100 & 0 \\
13 & 0 & 1 & 100 & 100 & 100 & 69 & 51 & 100 & 0 \\
12 & 0 & 1 & 100 & 100 & 100 & 69 & 51 & 100 & 0 \\
11 & 1 & 1 & 100 & 100 & 100 & 69 & 51 & 100 & 0 \\
8 & 1 & 3 & 100 & 100 & 100 & 73 & 51 & 100 & 0 \\
7 & 8 & 5 & 100 & 100 & 100 & 86 & 51 & 100 & 0 \\
6 & 8 & 11 & 100 & 100 & 100 & 90 & 51 & 100 & 5 \\
5 & 21 & 28 & 100 & 100 & 100 & 96 & 65 & 100 & 42 \\
4 & 37 & 61 & 100 & 100 & 100 & 100 & 65 & 100 & 71 \\
3 & 86 & 90 & 100 & 100 & 100 & 100 & 83 & 100 & 87 \\
2 & 95 & 97 & 100 & 100 & 100 & 100 & 83 & 100 & 92 \\
1 & 100 & 100 & 100 & 100 & 100 & 100 & 100 & 100 & 100 \\
\hline
\end{tabular}


C219. cum WCR-82H MOCNESS tow copepods

$4 k-3$

\begin{tabular}{|c|c|c|c|c|c|c|c|c|c|}
\hline net\# & \%fmim & \%fmty & \%faar & smaar & offege & \%fgmi & \%feme & smeme & \&fupl \\
\hline 18 & 0 & 0 & 0 & 0 & 0 & 0 & 0 & 0 & 0 \\
\hline 17 & 0 & 0 & 0 & 0 & 0 & 0 & 3 & 0 & 0 \\
\hline 16 & 0 & 0 & 0 & 0 & 67 & 0 & 3 & 0 & 0 \\
\hline 15 & 0 & 0 & 0 & 61 & 90 & 0 & 3 & 0 & 0 \\
\hline 14 & 0 & 0 & 0 & 89 & 91 & 0 & 3 & 0 & 0 \\
\hline 13 & 0 & 0 & 0 & 91 & 93 & 0 & 3 & 0 & 0 \\
\hline 12 & 0 & 0 & 0 & 98 & 94 & 0 & 3 & 0 & 0 \\
\hline 11 & 0 & 0 & 0 & 100 & 95 & 0 & 3 & 0 & 0 \\
\hline 8 & 0 & 0 & 0 & 100 & 100 & 0 & 3 & 0 & 0 \\
\hline 7 & 5 & 0 & 0 & 100 & 100 & 0 & 3 & 0 & 0 \\
\hline 6 & 34 & 0 & 0 & 100 & 100 & 70 & 3 & 0 & 0 \\
\hline 5 & 52 & 0 & 0 & 100 & 100 & 79 & 11 & 0 & 4 \\
\hline 4 & 75 & 0 & 30 & 100 & 100 & 92 & 58 & 40 & 55 \\
\hline 3 & 86 & 0 & 83 & 100 & 100 & 100 & 75 & 88 & 84 \\
\hline 2 & 90 & 25 & 100 & 100 & 100 & 100 & 93 & 100 & 93 \\
\hline 1 & 100 & 100 & 100 & 100 & 100 & 100 & 100 & 100 & 100 \\
\hline
\end{tabular}

net\# \%mupl \%fumj \%fchi \%fmed ommed ofmar ommar \%finc sminc

\begin{tabular}{|c|c|c|c|c|c|c|c|c|c|}
\hline 18 & 0 & 0 & 0 & 0 & 0 & 30 & 38 & 0 & 0 \\
\hline 17 & 0 & 0 & 0 & 0 & 0 & 51 & 38 & 0 & 0 \\
\hline 16 & 0 & 0 & 0 & 0 & 0 & 100 & 100 & 0 & 0 \\
\hline 15 & 0 & 0 & 0 & 2 & 0 & 100 & 100 & 0 & 0 \\
\hline 14 & 0 & 0 & 0 & 2 & 0 & 100 & 100 & 0 & 0 \\
\hline 13 & 0 & 0 & 0 & 2 & 0 & 100 & 100 & 0 & 0 \\
\hline 12 & 0 & 0 & 0 & 3 & 0 & 100 & 100 & 0 & 0 \\
\hline 11 & 0 & 0 & 0 & 3 & 0 & 100 & 100 & 0 & 0 \\
\hline 8 & 0 & 0 & 0 & 6 & 0 & 100 & 100 & 0 & 0 \\
\hline 7 & 0 & 0 & 0 & 10 & 0 & 100 & 100 & 0 & 0 \\
\hline 6 & 11 & 0 & 0 & 18 & 24 & 100 & 100 & 0 & 0 \\
\hline 5 & 17 & 0 & 0 & 75 & 84 & 100 & 100 & 0 & 0 \\
\hline 4 & 52 & 77 & 33 & 100 & 100 & 100 & 100 & 0 & 0 \\
\hline 3 & 87 & 100 & 81 & 100 & 100 & 100 & 100 & 100 & 80 \\
\hline 2 & 100 & 100 & 100 & 100 & 100 & 100 & 100 & 100 & 100 \\
\hline 1 & 100 & 100 & 100 & 100 & 100 & 100 & 100 & 100 & 100 \\
\hline
\end{tabular}


c219. cum WCR-82H MOCNESS tow copepods

$4 \mathrm{k}-4$

net\# \&ceuk \%fpsp ofslo ofsam ofscu ofsma omscp ofbra ombra

\begin{tabular}{rrrrrrrrrr}
\hline 18 & 25 & 0 & 0 & 0 & 0 & 0 & 0 & 0 & 0 \\
17 & 39 & 0 & 0 & 0 & 0 & 0 & 0 & 0 & 0 \\
16 & 44 & 0 & 0 & 0 & 0 & 0 & 0 & 0 & 0 \\
15 & 61 & 0 & 0 & 0 & 0 & 0 & 0 & 36 & 47 \\
14 & 69 & 51 & 0 & 0 & 0 & 0 & 0 & 44 & 47 \\
13 & 74 & 51 & 0 & 0 & 0 & 0 & 0 & 55 & 47 \\
12 & 76 & 51 & 0 & 0 & 0 & 0 & 0 & 59 & 51 \\
11 & 77 & 100 & 0 & 1 & 0 & 0 & 0 & 62 & 51 \\
8 & 82 & 100 & 0 & 1 & 0 & 0 & 0 & 62 & 51 \\
7 & 89 & 100 & 0 & 4 & 0 & 0 & 0 & 100 & 51 \\
6 & 92 & 100 & 10 & 53 & 29 & 0 & 17 & 100 & 75 \\
5 & 94 & 100 & 75 & 95 & 55 & 0 & 27 & 100 & 100 \\
4 & 95 & 100 & 87 & 100 & 73 & 63 & 31 & 100 & 100 \\
3 & 98 & 100 & 93 & 100 & 100 & 63 & 95 & 100 & 100 \\
2 & 98 & 100 & 99 & 100 & 100 & 100 & 100 & 100 & 100 \\
1 & 100 & 100 & 100 & 100 & 100 & 100 & 100 & 100 & 100 \\
\hline
\end{tabular}

\begin{tabular}{|c|c|c|c|c|c|c|c|c|c|}
\hline net\# & \%fdan & omdan & $\%$ fden & ofvit & $8 \mathrm{msca}$ & offst & \&ftmt & \%fven & omven \\
\hline $\begin{array}{r}18 \\
17 \\
16 \\
15 \\
14 \\
13 \\
12 \\
11 \\
8 \\
7 \\
6 \\
5 \\
4 \\
3 \\
2 \\
1\end{array}$ & $\begin{array}{r}70 \\
100 \\
100 \\
100 \\
100 \\
1000 \\
1000 \\
1000 \\
1000 \\
100 \\
100 \\
100 \\
1000 \\
1000 \\
100 \\
1000\end{array}$ & $\begin{array}{l}100 \\
100 \\
100 \\
100 \\
100 \\
100 \\
100 \\
100 \\
100 \\
100 \\
100 \\
100 \\
100 \\
100 \\
100 \\
100\end{array}$ & $\begin{array}{r}0 \\
0 \\
0 \\
0 \\
2 \\
2 \\
4 \\
6 \\
6 \\
12 \\
59 \\
79 \\
100 \\
100 \\
100 \\
100\end{array}$ & $\begin{array}{r}0 \\
0 \\
0 \\
0 \\
17 \\
26 \\
30 \\
38 \\
79 \\
100 \\
100 \\
100 \\
100 \\
100 \\
100 \\
100\end{array}$ & $\begin{array}{r}0 \\
0 \\
3 \\
5 \\
7 \\
8 \\
8 \\
10 \\
12 \\
14 \\
28 \\
37 \\
81 \\
96 \\
96 \\
100\end{array}$ & $\begin{array}{r}21 \\
66 \\
100 \\
100 \\
100 \\
100 \\
100 \\
100 \\
100 \\
100 \\
100 \\
100 \\
100 \\
100 \\
100 \\
100\end{array}$ & $\begin{array}{r}0 \\
0 \\
0 \\
0 \\
0 \\
0 \\
0 \\
0 \\
0 \\
0 \\
0 \\
0 \\
0 \\
34 \\
67 \\
100\end{array}$ & $\begin{array}{r}0 \\
0 \\
0 \\
0 \\
0 \\
0 \\
0 \\
0 \\
0 \\
0 \\
0 \\
1 \\
5 \\
49 \\
79 \\
100\end{array}$ & $\begin{array}{r}0 \\
0 \\
0 \\
0 \\
0 \\
0 \\
0 \\
0 \\
0 \\
0 \\
0 \\
2 \\
6 \\
73 \\
96 \\
100\end{array}$ \\
\hline
\end{tabular}


c219. cum WCR-82H MOCNESS tow copepods

$4 k-5$

\begin{tabular}{|c|c|c|c|c|c|c|c|c|c|}
\hline net\# & \&fbrv & \%mbrv & smlon & ocmet & \&fgra & ofmgra & \%fpis & ompis & \%cpsm \\
\hline 18 & 0 & 0 & 0 & 0 & 0 & 0 & 0 & 0 & $\overline{0}$ \\
\hline 17 & 0 & 0 & 0 & 0 & 1 & 0 & 0 & 1 & 0 \\
\hline 16 & 0 & 0 & 0 & 0 & 1 & 0 & 0 & 1 & 0 \\
\hline 15 & 0 & 0 & 0 & 0 & 1 & 0 & 0 & 1 & 0 \\
\hline 14 & 0 & 1 & 0 & 0 & 1 & 0 & 0 & 1 & 1 \\
\hline 13 & 0 & 1 & 0 & 0 & 1 & 0 & 0 & 1 & 1 \\
\hline 12 & 0 & 1 & 0 & 0 & 1 & 0 & 0 & 1 & 2 \\
\hline 11 & 0 & 1 & 0 & 0 & 1 & 1 & 1 & 1 & 9 \\
\hline 8 & 0 & 1 & 0 & 0 & 47 & 23 & 56 & 56 & 64 \\
\hline 7 & 0 & 1 & 0 & 0 & 72 & 29 & 89 & 75 & 77 \\
\hline 6 & 0 & 1 & 0 & 0 & 88 & 73 & 89 & 77 & 89 \\
\hline 5 & 0 & 1 & 0 & 1 & 99 & 99 & 93 & 82 & 94 \\
\hline 4 & 0 & 1 & 0 & 39 & 100 & 100 & 100 & 100 & 100 \\
\hline 3 & 51 & 10 & 34 & 91 & 100 & 100 & 100 & 100 & 100 \\
\hline 2 & 79 & 98 & 67 & 94 & 100 & 100 & 100 & 100 & 100 \\
\hline 1 & 100 & 100 & 100 & 100 & 100 & 100 & 100 & 100 & 100 \\
\hline
\end{tabular}

\begin{tabular}{|c|c|c|c|c|c|c|c|c|c|}
\hline net\# & \&fabd & \%mabd & ofxip & omxip & \%cplg & ofvio & smvio & oflcl & omlcl \\
\hline 18 & 1 & 0 & 0 & 0 & 0 & 38 & 51 & 0 & 0 \\
\hline 17 & 2 & 0 & 0 & 0 & 2 & 38 & 78 & 0 & 0 \\
\hline 16 & 2 & 0 & 0 & 0 & 3 & 100 & 78 & 0 & 0 \\
\hline 15 & 2 & 0 & 0 & 0 & 6 & 100 & 78 & 0 & 0 \\
\hline 14 & 2 & 1 & 0 & 1 & 11 & 100 & 78 & 0 & 0 \\
\hline 13 & 2 & 1 & 0 & 1 & 13 & 100 & 78 & 0 & 0 \\
\hline 12 & 2 & 1 & 0 & 1 & 14 & 100 & 78 & 2 & 1 \\
\hline 11 & 2 & 1 & 0 & 1 & 14 & 100 & 78 & 6 & 3 \\
\hline 8 & 3 & 3 & 1 & 1 & 27 & 100 & 78 & 19 & 4 \\
\hline 7 & 23 & 6 & 1 & 5 & 65 & 100 & 78 & 63 & 57 \\
\hline 6 & 62 & 43 & $\overline{1}$ & 5 & 76 & 100 & 78 & 100 & 100 \\
\hline 5 & 87 & 62 & 85 & 56 & 80 & 100 & 100 & 100 & 100 \\
\hline 4 & 93 & 83 & 92 & 74 & 90 & 100 & 100 & 100 & 100 \\
\hline 3 & 96 & 85 & 96 & 82 & 97 & 100 & 100 & 100 & 100 \\
\hline 2 & 100 & 91 & 99 & 100 & 99 & 100 & 100 & 100 & 100 \\
\hline 1 & 100 & 100 & 100 & 100 & 100 & 100 & 100 & 100 & 100 \\
\hline
\end{tabular}


C219. cum WCR-82H MOCNESS tow copepods

$4 k-6$

\begin{tabular}{|c|c|c|c|c|c|c|c|c|c|}
\hline net\# & oflut & otmlut & \%fpap & \&ompap & $8 f s p i$ & 8mspi & ofvip & ofmvip & ofhet \\
\hline 18 & 1 & 0 & 4 & 5 & 0 & 0 & 0 & 0 & 0 \\
\hline 17 & 6 & 9 & 14 & 30 & 0 & 0 & 0 & 0 & 0 \\
\hline 16 & 40 & 56 & 21 & 47 & 0 & 0 & 0 & 0 & 0 \\
\hline 15 & 59 & 79 & 23 & 54 & 0 & 0 & 0 & 0 & 0 \\
\hline 14 & 65 & 84 & 25 & 56 & 0 & 0 & 0 & 0 & 0 \\
\hline 13 & 69 & 86 & 26 & 60 & 2 & 5 & 0 & 0 & 0 \\
\hline 12 & 73 & 86 & 27 & 63 & 2 & 5 & 0 & 0 & 0 \\
\hline 11 & 76 & 87 & 28 & 68 & 4 & 9 & 0 & 0 & 0 \\
\hline 8 & 88 & 94 & 32 & 80 & 4 & 9 & 0 & 0 & 0 \\
\hline 7 & 93 & 95 & 49 & 87 & 53 & 52 & 11 & 7 & 0 \\
\hline 6 & 96 & 95 & 77 & 96 & 53 & 100 & 31 & 30 & 24 \\
\hline 5 & 98 & 96 & 90 & 100 & 53 & 100 & 31 & 30 & 30 \\
\hline 4 & 98 & 96 & 93 & 100 & 87 & 100 & 50 & 38 & 36 \\
\hline 3 & 99 & 97 & 97 & 100 & 100 & 100 & 62 & 100 & 54 \\
\hline 2 & 100 & 100 & 98 & 100 & 100 & 100 & 85 & 100 & 96 \\
\hline 1 & 100 & 100 & 100 & 100 & 100 & 100 & 100 & 100 & 100 \\
\hline
\end{tabular}

net\# \%mhet ochet \%fhal smhal schal \%forn sfaug \%fhel \%mhel

\begin{tabular}{rrrrrrrrrr}
\hline 18 & 0 & 0 & 0 & 0 & 0 & 0 & 0 & 0 & 0 \\
17 & 0 & 5 & 0 & 0 & 0 & 0 & 0 & 0 & 0 \\
16 & 0 & 21 & 50 & 55 & 2 & 4 & 6 & 0 & 0 \\
15 & 0 & 25 & 54 & 78 & 11 & 8 & 6 & 0 & 0 \\
14 & 0 & 29 & 56 & 89 & 42 & 9 & 8 & 0 & 0 \\
13 & 0 & 30 & 57 & 93 & 55 & 9 & 10 & 0 & 0 \\
12 & 0 & 32 & 58 & 94 & 59 & 9 & 11 & 0 & 0 \\
11 & 0 & 36 & 58 & 94 & 64 & 9 & 11 & 0 & 0 \\
8 & 0 & 54 & 60 & 94 & 83 & 14 & 12 & 0 & 0 \\
7 & 0 & 69 & 77 & 100 & 86 & 17 & 35 & 0 & 0 \\
6 & 10 & 88 & 91 & 100 & 97 & 53 & 37 & 0 & 0 \\
5 & 10 & 93 & 97 & 100 & 100 & 91 & 42 & 12 & 15 \\
4 & 21 & 93 & 100 & 100 & 100 & 100 & 54 & 61 & 76 \\
3 & 64 & 98 & 100 & 100 & 100 & 100 & 86 & 93 & 91 \\
2 & 94 & 98 & 100 & 100 & 100 & 100 & 97 & 96 & 94 \\
1 & 100 & 100 & 100 & 100 & 100 & 100 & 100 & 100 & 100 \\
\hline
\end{tabular}


c219. cum WCR-82H MOCNESS tow copepods

$4 k-7$

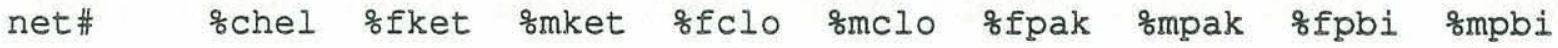

\begin{tabular}{|c|c|c|c|c|c|c|c|c|c|}
\hline 18 & 0 & 0 & 0 & 0 & 0 & 0 & 88 & 0 & 17 \\
\hline 17 & 0 & 0 & 0 & 0 & 0 & 29 & 88 & 41 & 65 \\
\hline 16 & 0 & 0 & 0 & 0 & 0 & 29 & 88 & 90 & 99 \\
\hline 15 & 0 & 0 & 0 & 0 & 0 & 52 & 88 & 95 & 99 \\
\hline 14 & 0 & 0 & 0 & 0 & 0 & 52 & 88 & 96 & 99 \\
\hline 13 & 0 & 0 & 0 & 0 & 0 & 52 & 88 & 96 & 99 \\
\hline 12 & 0 & 0 & 0 & 0 & 0 & 52 & 88 & 96 & 100 \\
\hline 11 & 0 & 0 & 0 & 0 & 2 & 52 & 100 & 96 & 100 \\
\hline 8 & 0 & 0 & 0 & 0 & 2 & 52 & 100 & 96 & 100 \\
\hline 7 & 2 & 0 & 0 & 0 & 2 & 52 & 100 & 96 & 100 \\
\hline 6 & 23 & 72 & 40 & 0 & 2 & 52 & 100 & 100 & 100 \\
\hline 5 & 49 & 100 & 100 & 100 & 100 & 52 & 100 & 100 & 100 \\
\hline 4 & 64 & 100 & 100 & 100 & 100 & 100 & 100 & 100 & 100 \\
\hline 3 & 93 & 100 & 100 & 100 & 100 & 100 & 100 & 100 & 100 \\
\hline 2 & 94 & 100 & 100 & 100 & 100 & 100 & 100 & 100 & 100 \\
\hline 1 & 100 & 100 & 100 & 100 & 100 & 100 & 100 & 100 & 100 \\
\hline
\end{tabular}

net\# ofpon \%fada offneg \%fmis ommis ocmis soith oharp ototl

\begin{tabular}{|c|c|c|c|c|c|c|c|c|c|}
\hline 18 & 41 & 0 & 53 & 0 & 3 & 5 & 2 & 21 & 6 \\
\hline 17 & 55 & 0 & 69 & 2 & 3 & 11 & 5 & 38 & 13 \\
\hline 16 & 100 & 0 & 95 & 30 & 15 & 19 & 50 & 53 & 25 \\
\hline 15 & 100 & 0 & 95 & 34 & 15 & 21 & 75 & 63 & 31 \\
\hline 14 & 100 & 0 & 95 & 35 & 15 & 23 & 79 & 69 & 35 \\
\hline 13 & 100 & 0 & 97 & 35 & 17 & 24 & 79 & 72 & 37 \\
\hline 12 & 100 & 26 & 97 & 36 & 17 & 24 & 79 & 74 & 38 \\
\hline 11 & 100 & 26 & 98 & 36 & 19 & 25 & 81 & 75 & 40 \\
\hline 8 & 100 & 26 & 98 & 37 & 27 & 29 & 82 & 78 & 48 \\
\hline 7 & 100 & 26 & 98 & 46 & 27 & 33 & 90 & 79 & 55 \\
\hline 6 & 100 & 26 & 98 & 52 & 42 & 54 & 97 & 81 & 66 \\
\hline 5 & 100 & 100 & 98 & 57 & 45 & 65 & 98 & 85 & 75 \\
\hline 4 & 100 & 100 & 98 & 68 & 51 & 76 & 99 & 94 & 83 \\
\hline 3 & 100 & 100 & 98 & 77 & 79 & 86 & 100 & 96 & 90 \\
\hline 2 & 100 & 100 & 98 & 85 & 95 & 94 & 100 & 98 & 96 \\
\hline 1 & 100 & 100 & 100 & 100 & 100 & 100 & 100 & 100 & 100 \\
\hline
\end{tabular}


mocness-1-cop220.raw WCR-82H MOCNESS tow copepods

$4 \mathrm{~L}-1$

\begin{tabular}{cccccccccc} 
net\# & midd & depi & volf & aliq & ften & mten & cten & fnro & mnro \\
\hline 0 & 50 & 100 & 650 & 8 & 31 & 6 & 18 & 1 & 0 \\
1 & 50 & 100 & 528 & 8 & 23 & 3 & 13 & 1 & 0 \\
2 & 50 & 100 & 493 & 8 & 18 & 8 & 21 & 0 & 0 \\
3 & 50 & 100 & 505 & 8 & 30 & 11 & 17 & 0 & 0 \\
4 & 50 & 100 & 587 & 8 & 36 & 6 & 28 & 0 & 0 \\
5 & 50 & 100 & 644 & 8 & 46 & 4 & 16 & 2 & 0 \\
6 & 50 & 100 & 599 & 8 & 16 & 0 & 16 & 0 & 0 \\
7 & 50 & 100 & 542 & 8 & 27 & 4 & 37 & 1 & 1 \\
8 & 50 & 100 & 583 & 16 & 13 & 4 & 25 & 1 & 0 \\
11 & 50 & 100 & 591 & 16 & 30 & 6 & 27 & 1 & 0 \\
12 & 50 & 100 & 562 & 16 & 19 & 3 & 24 & 1 & 0 \\
13 & 50 & 100 & 697 & 16 & 31 & 2 & 17 & 0 & 0 \\
14 & 50 & 100 & 536 & 16 & 23 & 1 & 25 & 0 & 0 \\
15 & 50 & 100 & 548 & 16 & 11 & 1 & 13 & 0 & 0 \\
16 & 50 & 100 & 561 & 16 & 21 & 1 & 19 & 0 & 0 \\
17 & 50 & 100 & 511 & 16 & 17 & 4 & 16 & 0 & 0 \\
18 & 50 & 100 & 549 & 16 & 28 & 0 & 26 & 0 & 0 \\
19 & 50 & 100 & 584 & 16 & 31 & 3 & 28 & 0 & 0 \\
\hline
\end{tabular}

net\# fngr cneo fund mund cund ccal fmin mmin cmin

\begin{tabular}{rrrrrrrrrr}
\hline 0 & 1 & 11 & 12 & 12 & 89 & 6 & 21 & 10 & 79 \\
1 & 2 & 20 & 9 & 10 & 24 & 11 & 35 & 15 & 69 \\
2 & 2 & 15 & 8 & 5 & 24 & 20 & 34 & 5 & 53 \\
3 & 0 & 26 & 16 & 19 & 14 & 19 & 56 & 20 & 48 \\
4 & 1 & 31 & 17 & 8 & 25 & 16 & 57 & 24 & 33 \\
5 & 4 & 20 & 13 & 16 & 23 & 23 & 49 & 24 & 37 \\
6 & 7 & 37 & 31 & 17 & 34 & 15 & 40 & 10 & 41 \\
7 & 3 & 35 & 6 & 6 & 6 & 20 & 57 & 31 & 59 \\
8 & 6 & 26 & 15 & 2 & 19 & 12 & 36 & 16 & 47 \\
11 & 0 & 13 & 1 & 2 & 11 & 13 & 21 & 14 & 25 \\
12 & 1 & 18 & 2 & 1 & 16 & 8 & 26 & 19 & 30 \\
13 & 1 & 8 & 3 & 2 & 31 & 4 & 8 & 7 & 44 \\
14 & 1 & 5 & 3 & 0 & 20 & 6 & 8 & 4 & 30 \\
15 & 3 & 19 & 5 & 9 & 2 & 8 & 12 & 5 & 14 \\
16 & 2 & 15 & 6 & 8 & 7 & 15 & 42 & 17 & 21 \\
17 & 0 & 12 & 11 & 12 & 11 & 3 & 19 & 7 & 28 \\
18 & 0 & 17 & 12 & 6 & 29 & 18 & 19 & 17 & 39 \\
19 & 6 & 23 & 19 & 26 & 40 & 24 & 35 & 20 & 60 \\
\hline
\end{tabular}


mocness-1-cop220.raw WCR-82H MOCNESS tow copepods

$4 \mathrm{~L}-2$

\begin{tabular}{|c|c|c|c|c|c|c|c|c|c|}
\hline net\# & fatt & matt & catt & fmon & cmon & fmey & fcor & $\operatorname{ccor}$ & facr \\
\hline 0 & 3 & 0 & 11 & 5 & 0 & 0 & 1 & 0 & 48 \\
\hline 1 & 4 & 2 & 9 & 0 & 0 & 0 & 0 & 0 & 12 \\
\hline 2 & 6 & 0 & 17 & 0 & 0 & 0 & 0 & 0 & 15 \\
\hline 3 & 3 & 0 & 22 & 1 & 0 & 0 & 0 & 1 & 8 \\
\hline 4 & 2 & 0 & 25 & 0 & 0 & 0 & 0 & 0 & 6 \\
\hline 5 & 0 & 0 & 24 & 0 & 2 & 0 & 0 & 1 & 7 \\
\hline 6 & 4 & 0 & 25 & 0 & 0 & 0 & 0 & 1 & 7 \\
\hline 7 & 3 & 1 & 44 & 0 & 0 & 0 & 0 & 2 & 9 \\
\hline 8 & 1 & 0 & 26 & 0 & 0 & 1 & 0 & 0 & 9 \\
\hline 11 & 2 & 0 & 23 & 0 & 2 & 1 & 0 & 0 & 9 \\
\hline 12 & 3 & 0 & 19 & 0 & 0 & 1 & 0 & 1 & 4 \\
\hline 13 & 2 & 0 & 9 & 2 & 0 & 0 & 0 & 0 & 5 \\
\hline 14 & 2 & 1 & 4 & 0 & 5 & 1 & 0 & 0 & 16 \\
\hline 15 & 3 & 0 & 21 & 0 & 0 & 0 & 0 & 2 & 3 \\
\hline 16 & 2 & 2 & 14 & 1 & 7 & 0 & 0 & 0 & 8 \\
\hline 17 & 0 & 0 & 6 & 2 & 1 & 0 & 0 & 0 & 5 \\
\hline 18 & 0 & 1 & 9 & 0 & 1 & 2 & 0 & 0 & 18 \\
\hline 19 & 2 & 0 & 12 & 0 & 1 & 7 & 0 & 1 & 27 \\
\hline
\end{tabular}

\begin{tabular}{|c|c|c|c|c|c|c|c|c|c|}
\hline net\# & faca & fprv & farc & marc & carc & furc & faar & maar & fege \\
\hline 0 & 0 & 0 & 58 & 1 & 17 & 1 & 0 & 3 & 5 \\
\hline 1 & 2 & 0 & 47 & 6 & 12 & 1 & 0 & 1 & 5 \\
\hline 2 & 2 & 1 & 41 & 0 & 12 & 0 & 0 & 1 & 5 \\
\hline 3 & 3 & 0 & 31 & 2 & 9 & 0 & 0 & 0 & 10 \\
\hline 4 & 3 & 1 & 43 & 4 & 7 & 1 & 0 & 3 & 5 \\
\hline 5 & 0 & 0 & 46 & 2 & 14 & 1 & 0 & 0 & 11 \\
\hline 6 & 2 & 0 & 34 & 0 & 1 & 1 & 0 & 1 & 4 \\
\hline 7 & 1 & 0 & 57 & 2 & 5 & 1 & 0 & 2 & 3 \\
\hline 8 & 0 & 2 & 65 & 1 & 17 & 3 & 0 & 1 & 5 \\
\hline 11 & 0 & 0 & 28 & 1 & 3 & 1 & 0 & 4 & 3 \\
\hline 12 & 0 & 0 & 48 & 3 & 4 & 0 & 0 & 1 & 4 \\
\hline 13 & 1 & 0 & 16 & 0 & 6 & 0 & 0 & 8 & 6 \\
\hline 14 & 0 & 1 & 48 & 2 & 13 & 0 & 0 & 3 & 3 \\
\hline 15 & 1 & 0 & 13 & 2. & 1 & 0 & 0 & 2 & 0 \\
\hline 16 & 0 & 2 & 20 & 0 & 1 & 0 & 1 & 0 & 10 \\
\hline 17 & 0 & 0 & 23 & 0 & 0 & 0 & 0 & 0 & 3 \\
\hline 18 & 0 & 0 & 21 & 0 & 4 & 1 & 1 & 0 & 2 \\
\hline 19 & 0 & 8 & 72 & 5 & 24 & 8 & 0 & 0 & 3 \\
\hline
\end{tabular}


mocness-1-cop220.raw WCR-82H MOCNESS tow copepods

$4 l-3$

\begin{tabular}{|c|c|c|c|c|c|c|c|c|c|}
\hline net\# & cege : & feme & meme & cuch & fchi & mchi & fupl & mupl & cupl \\
\hline 0 & 13 & 0 & 0 & 5 & 0 & 0 & 0 & 0 & 0 \\
\hline 1 & 4 & 0 & 0 & 6 & 0 & 0 & 0 & 0 & 0 \\
\hline 2 & 7 & 0 & 0 & 6 & 0 & 0 & 0 & 0 & 0 \\
\hline 3 & 3 & 0 & 0 & 9 & 0 & 0 & 0 & 0 & 0 \\
\hline 4 & 6 & 0 & 0 & 14 & 0 & 0 & 0 & 0 & 0 \\
\hline 5 & 2 & 0 & 0 & 13 & 0 & 0 & 0 & 0 & 0 \\
\hline 6 & 3 & 0 & 0 & 9 & 0 & 0 & 0 & 0 & 0 \\
\hline 7 & 3 & 0 & 0 & 13 & 0 & 0 & 0 & 0 & 0 \\
\hline 8 & 4 & 0 & 0 & 10 & 0 & 0 & 0 & 0 & 0 \\
\hline 11 & 1 & 0 & 0 & 12 & 0 & 0 & 0 & 0 & 0 \\
\hline 12 & 6 & 0 & 0 & 16 & 0 & 0 & 1 & 0 & 0 \\
\hline 13 & 3 & 0 & 0 & 17 & 0 & 0 & 2 & 0 & 3 \\
\hline 14 & 2 & 0 & 0 & 14 & 0 & 0 & 1 & 0 & 5 \\
\hline 15 & 5 & 0 & 0 & 12 & 0 & 0 & 0 & 0 & 7 \\
\hline 16 & 13 & 1 & 0 & 24 & 0 & 0 & 3 & 2 & 20 \\
\hline 17 & 5 & 2 & 0 & 10 & 0 & 0 & 2 & 3 & 23 \\
\hline 18 & 5 & 0 & 0 & 18 & 0 & 0 & 8 & 1 & 20 \\
\hline 19 & 11 & 2 & 0 & 40 & 0 & 0 & 5 & 1 & 20 \\
\hline
\end{tabular}

\begin{tabular}{crrrrrrrrr} 
net\# & fmar & mmar & feuk & meuk & ceuk & fpsp & cpsp & flop & clop \\
\hline 0 & 3 & 1 & 0 & 0 & 129 & 0 & 3 & 1 & 0 \\
1 & 0 & 2 & 6 & 1 & 157 & 0 & 1 & 3 & 0 \\
2 & 2 & 1 & 0 & 0 & 117 & 0 & 2 & 1 & 0 \\
3 & 8 & 3 & 0 & 0 & 148 & 0 & 0 & 2 & 0 \\
4 & 11 & 9 & 1 & 0 & 152 & 1 & 4 & 2 & 0 \\
5 & 10 & 3 & 0 & 0 & 164 & 0 & 0 & 3 & 0 \\
6 & 15 & 2 & 0 & 0 & 113 & 2 & 3 & 2 & 0 \\
7 & 15 & 8 & 0 & 0 & 150 & 0 & 2 & 2 & 0 \\
8 & 16 & 9 & 0 & 0 & 86 & 0 & 0 & 4 & 0 \\
11 & 6 & 0 & 0 & 0 & 66 & 0 & 0 & 2 & 0 \\
12 & 5 & 0 & 0 & 0 & 80 & 0 & 1 & 2 & 0 \\
13 & 2 & 1 & 0 & 2 & 140 & 0 & 1 & 2 & 0 \\
14 & 5 & 0 & 0 & 0 & 91 & 0 & 2 & 1 & 0 \\
15 & 5 & 0 & 1 & 0 & 63 & 0 & 5 & 6 & 0 \\
16 & 10 & 1 & 1 & 0 & 90 & 0 & 3 & 3 & 4 \\
17 & 2 & 1 & 0 & 0 & 91 & 0 & 4 & 1 & 1 \\
18 & 7 & 2 & 1 & 0 & 93 & 1 & 1 & 1 & 3 \\
19 & 9 & 3 & 4 & 0 & 144 & 1 & 1 & 2 & 2 \\
\hline
\end{tabular}


mocness-1-cop220.raw WCR-82H MOCNESS tow copepods

$4 l-4$

\begin{tabular}{|c|c|c|c|c|c|c|c|c|c|}
\hline net\# & fsam & csam & fslo & fbra & mbra & cbra & fdan & mdan & cdan \\
\hline 0 & 0 & 1 & 0 & 1 & 3 & 7 & 6 & 9 & 39 \\
\hline 1 & 0 & 1 & 0 & 3 & 1 & 7 & 5 & 2 & 33 \\
\hline 2 & 0 & 1 & 0 & 4 & 1 & 7 & 2 & 0 & 31 \\
\hline 3 & 0 & 2 & 1 & 3 & 1 & 7 & 0 & 3 & 41 \\
\hline 4 & 0 & 3 & 0 & 4 & 0 & 8 & 2 & 1 & 35 \\
\hline 5 & 0 & 1 & 0 & 5 & 0 & 8 & 1 & 2 & 26 \\
\hline 6 & 0 & 2 & 0 & 2 & 0 & 4 & 4 & 1 & 6 \\
\hline 7 & 0 & 4 & 0 & 3 & 0 & 2 & 2 & 1 & 12 \\
\hline 8 & 0 & 0 & 0 & 0 & 1 & 3 & 0 & 3 & 12 \\
\hline 11 & 0 & 0 & 0 & 3 & 2 & 4 & 0 & 0 & 3 \\
\hline 12 & 0 & 1 & 0 & 3 & 2 & 8 & 0 & 1 & 7 \\
\hline 13 & 2 & 2 & 0 & 5 & 2 & 2 & 2 & 0 & 19 \\
\hline 14 & 2 & 3 & 1 & 4 & 0 & 5 & 0 & 0 & 10 \\
\hline 15 & 1 & 2 & 0 & 2 & 1 & 6 & 0 & 0 & 8 \\
\hline 16 & 4 & 3 & 2 & 4 & 2 & 6 & 1 & 0 & 0 \\
\hline 17 & 8 & 7 & 0 & 3 & 1 & 2 & 1 & 3 & 10 \\
\hline 18 & 8 & 14 & 3 & 5 & 0 & 8 & 0 & 2 & 12 \\
\hline 19 & 12 & 13 & 1 & 1 & 0 & 7 & 4 & 4 & 25 \\
\hline
\end{tabular}

\begin{tabular}{crrrrrrrrr} 
net\# & flam & clam & fvit & fden & mden & cden & ftst & mtst & ctst \\
\hline 0 & 5 & 1 & 0 & 0 & 0 & 1 & 1 & 0 & 9 \\
1 & 3 & 6 & 0 & 0 & 0 & 0 & 1 & 1 & 9 \\
2 & 2 & 4 & 0 & 0 & 0 & 1 & 0 & 1 & 6 \\
3 & 11 & 9 & 0 & 0 & 3 & 0 & 1 & 0 & 2 \\
4 & 9 & 5 & 0 & 0 & 4 & 1 & 3 & 0 & 10 \\
5 & 12 & 1 & 0 & 0 & 2 & 0 & 0 & 0 & 3 \\
6 & 4 & 0 & 0 & 2 & 0 & 0 & 0 & 1 & 4 \\
7 & 15 & 7 & 0 & 0 & 3 & 2 & 1 & 2 & 2 \\
8 & 8 & 1 & 0 & 0 & 0 & 1 & 1 & 1 & 2 \\
11 & 4 & 6 & 0 & 0 & 1 & 3 & 3 & 0 & 2 \\
12 & 12 & 3 & 0 & 1 & 1 & 0 & 0 & 0 & 1 \\
13 & 5 & 2 & 0 & 0 & 4 & 0 & 3 & 0 & 3 \\
14 & 3 & 2 & 0 & 2 & 2 & 1 & 1 & 0 & 5 \\
15 & 2 & 3 & 0 & 1 & 2 & 0 & 0 & 0 & 5 \\
16 & 8 & 1 & 0 & 5 & 2 & 4 & 1 & 0 & 2 \\
17 & 6 & 3 & 0 & 2 & 1 & 2 & 0 & 0 & 3 \\
18 & 1 & 0 & 0 & 11 & 2 & 6 & 0 & 2 & 1 \\
19 & 6 & 3 & 0 & 9 & 2 & 4 & 1 & 2 & 5 \\
\hline
\end{tabular}


mocness-1-cop220.raw WCR-82H MOCNESS tow copepods

$41-5$

\begin{tabular}{cccccccccc} 
net\# & fttu & mttu & fven & mven & fbrv & mbrv & mlon & fbor & fgra \\
\hline 0 & 0 & 0 & 0 & 0 & 0 & 0 & 0 & 0 & 0 \\
1 & 0 & 0 & 0 & 0 & 0 & 0 & 0 & 0 & 0 \\
2 & 0 & 0 & 0 & 0 & 0 & 0 & 0 & 0 & 0 \\
3 & 1 & 0 & 0 & 0 & 0 & 0 & 0 & 0 & 0 \\
4 & 0 & 1 & 0 & 0 & 0 & 0 & 0 & 0 & 1 \\
5 & 0 & 0 & 0 & 0 & 0 & 0 & 0 & 0 & 2 \\
6 & 0 & 0 & 0 & 0 & 0 & 0 & 0 & 0 & 0 \\
7 & 1 & 0 & 0 & 0 & 0 & 0 & 0 & 0 & 2 \\
8 & 1 & 0 & 0 & 0 & 0 & 0 & 0 & 0 & 6 \\
11 & 0 & 0 & 0 & 0 & 0 & 0 & 0 & 0 & 9 \\
12 & 1 & 0 & 0 & 0 & 0 & 0 & 0 & 0 & 19 \\
13 & 0 & 0 & 0 & 0 & 0 & 0 & 0 & 0 & 27 \\
14 & 1 & 0 & 0 & 0 & 0 & 0 & 0 & 0 & 30 \\
15 & 0 & 0 & 0 & 0 & 0 & 0 & 0 & 0 & 12 \\
16 & 0 & 0 & 0 & 0 & 0 & 0 & 0 & 0 & 29 \\
17 & 1 & 0 & 0 & 0 & 0 & 0 & 0 & 0 & 32 \\
18 & 0 & 0 & 0 & 0 & 0 & 0 & 0 & 0 & 38 \\
19 & 2 & 0 & 0 & 0 & 0 & 0 & 0 & 0 & 55 \\
\hline
\end{tabular}

\begin{tabular}{crrrrrrrrr} 
net\# & mpsm & fpis & mpis & cpsm & fabd & mabd & fxip & mxip & cplg \\
\hline 0 & 0 & 0 & 0 & 0 & 0 & 0 & 0 & 0 & 16 \\
1 & 0 & 0 & 0 & 0 & 0 & 0 & 0 & 0 & 53 \\
2 & 0 & 0 & 0 & 0 & 0 & 0 & 0 & 0 & 23 \\
3 & 0 & 0 & 0 & 0 & 0 & 0 & 0 & 0 & 19 \\
4 & 0 & 1 & 0 & 0 & 0 & 0 & 0 & 0 & 24 \\
5 & 0 & 0 & 0 & 1 & 0 & 0 & 0 & 0 & 30 \\
6 & 0 & 0 & 0 & 0 & 0 & 0 & 0 & 0 & 13 \\
7 & 1 & 0 & 0 & 1 & 0 & 0 & 0 & 0 & 0 \\
8 & 1 & 5 & 0 & 12 & 0 & 0 & 0 & 0 & 15 \\
11 & 0 & 0 & 0 & 13 & 0 & 0 & 0 & 0 & 17 \\
12 & 8 & 2 & 1 & 23 & 8 & 1 & 0 & 0 & 6 \\
13 & 13 & 9 & 6 & 34 & 5 & 0 & 0 & 0 & 27 \\
14 & 10 & 9 & 6 & 29 & 8 & 0 & 0 & 0 & 27 \\
15 & 5 & 12 & 6 & 29 & 12 & 0 & 0 & 0 & 31 \\
16 & 15 & 8 & 6 & 30 & 8 & 4 & 1 & 0 & 33 \\
17 & 14 & 11 & 3 & 35 & 13 & 1 & 2 & 1 & 41 \\
18 & 17 & 9 & 4 & 56 & 9 & 3 & 5 & 1 & 33 \\
19 & 18 & 10 & 11 & 79 & 23 & 10 & 3 & 2 & 62 \\
\hline
\end{tabular}


mocness-1-cop220.raw WCR-82H MOCNESS tow copepods

$4 l-6$

\begin{tabular}{crrrrrrrrr} 
net\# & fvio & mvio & cvio & ftyp & mtyp & flut & mlut & clut & fpap \\
\hline 0 & 1 & 0 & 4 & 0 & 0 & 87 & 36 & 1 & 6 \\
1 & 4 & 3 & 2 & 0 & 0 & 100 & 67 & 0 & 18 \\
2 & 1 & 0 & 1 & 0 & 0 & 78 & 60 & 1 & 4 \\
3 & 0 & 0 & 4 & 0 & 0 & 119 & 81 & 0 & 21 \\
4 & 2 & 0 & 0 & 0 & 0 & 81 & 65 & 1 & 23 \\
5 & 1 & 0 & 2 & 0 & 0 & 78 & 61 & 1 & 19 \\
6 & 0 & 0 & 0 & 0 & 0 & 35 & 27 & 0 & 11 \\
7 & 2 & 1 & 1 & 0 & 0 & 101 & 76 & 3 & 12 \\
8 & 0 & 1 & 0 & 0 & 0 & 78 & 44 & 1 & 7 \\
11 & 0 & 0 & 0 & 0 & 0 & 78 & 58 & 0 & 6 \\
12 & 1 & 0 & 0 & 0 & 0 & 82 & 66 & 2 & 4 \\
13 & 0 & 0 & 2 & 0 & 0 & 44 & 54 & 0 & 5 \\
14 & 1 & 1 & 2 & 0 & 0 & 57 & 31 & 1 & 6 \\
15 & 0 & 2 & 0 & 0 & 0 & 56 & 41 & 1 & 5 \\
16 & 0 & 0 & 1 & 0 & 0 & 49 & 52 & 1 & 5 \\
17 & 0 & 0 & 2 & 0 & 0 & 59 & 41 & 1 & 3 \\
18 & 0 & 2 & 3 & 0 & 0 & 82 & 40 & 2 & 0 \\
19 & 2 & 1 & 3 & 0 & 0 & 123 & 66 & 5 & 9 \\
\hline
\end{tabular}

\begin{tabular}{rrrrrrrrrr} 
net\# & mpap & cpap & fspi & mspi & cspi & fhal & mhal & chal & augs \\
\hline 0 & 9 & 8 & 0 & 0 & 0 & 110 & 3 & 12 & 0 \\
1 & 13 & 11 & 0 & 0 & 0 & 74 & 3 & 19 & 3 \\
2 & 17 & 19 & 0 & 0 & 0 & 63 & 4 & 21 & 8 \\
3 & 16 & 47 & 0 & 0 & 0 & 177 & 0 & 15 & 5 \\
4 & 18 & 35 & 0 & 0 & 0 & 115 & 7 & 17 & 6 \\
5 & 16 & 35 & 0 & 0 & 0 & 134 & 2 & 22 & 9 \\
6 & 6 & 11 & 0 & 0 & 0 & 67 & 1 & 12 & 6 \\
7 & 6 & 25 & 0 & 0 & 2 & 54 & 0 & 20 & 3 \\
8 & 6 & 8 & 0 & 0 & 0 & 31 & 3 & 20 & 6 \\
11 & 4 & 10 & 0 & 0 & 0 & 43 & 1 & 12 & 4 \\
12 & 5 & 13 & 0 & 0 & 0 & 46 & 2 & 10 & 9 \\
13 & 8 & 5 & 0 & 0 & 4 & 63 & 0 & 9 & 2 \\
14 & 5 & 8 & 0 & 0 & 0 & 30 & 1 & 7 & 4 \\
15 & 6 & 11 & 0 & 0 & 0 & 29 & 0 & 6 & 0 \\
16 & 3 & 6 & 0 & 0 & 5 & 32 & 0 & 11 & 6 \\
17 & 3 & 7 & 0 & 0 & 2 & 21 & 1 & 8 & 1 \\
18 & 5 & 10 & 0 & 0 & 1 & 15 & 0 & 15 & 2 \\
19 & 5 & 21 & 0 & 0 & 1 & 32 & 4 & 23 & 6 \\
\hline
\end{tabular}


mocness-1-cop220.raw WCR-82H MOCNESS tow copepods

$4 l-7$

net\# fhel mhel fcur mcur feth meth fpak mpak fpbi

\begin{tabular}{|c|c|c|c|c|c|c|c|c|c|}
\hline 0 & 0 & 0 & 0 & 0 & 0 & 0 & 2 & 1 & 9 \\
\hline 1 & 0 & 0 & 0 & 0 & 0 & 0 & 2 & 2 & 27 \\
\hline 2 & 0 & 0 & 0 & 0 & 0 & 0 & 0 & 1 & 19 \\
\hline 3 & 0 & 0 & 0 & 0 & 0 & 0 & 0 & 1 & 18 \\
\hline 4 & 0 & 0 & 0 & 0 & 1 & 0 & 0 & 1 & 8 \\
\hline 5 & 0 & 0 & 0 & 0 & 2 & 0 & 3 & 0 & 19 \\
\hline 6 & 0 & 0 & 0 & 0 & 1 & 0 & 0 & 0 & 24 \\
\hline 7 & 0 & 0 & 0 & 0 & 0 & 0 & 0 & 0 & 5 \\
\hline 8 & 0 & 0 & 0 & 0 & 0 & 0 & 2 & 2 & 16 \\
\hline 11 & 0 & 0 & 0 & 0 & 0 & 0 & 0 & 0 & 9 \\
\hline 12 & 0 & 0 & 0 & 0 & 0 & 0 & 1 & 1 & 14 \\
\hline 13 & 0 & 0 & 0 & 0 & 0 & 0 & 1 & 0 & 18 \\
\hline 14 & 0 & 0 & 0 & 0 & 0 & 0 & 0 & 0 & 9 \\
\hline 15 & 0 & 0 & 0 & 0 & 0 & 0 & 0 & 0 & 9 \\
\hline 16 & 0 & 0 & 0 & 0 & 0 & 0 & 0 & 0 & 16 \\
\hline 17 & 0 & 0 & 0 & 1 & 0 & 0 & 0 & 0 & 6 \\
\hline 18 & 0 & 0 & 0 & 0 & 0 & 0 & 2 & 0 & 16 \\
\hline 19 & 0 & 0 & 0 & 1 & 0 & 1 & 1 & 3 & 14 \\
\hline
\end{tabular}

\begin{tabular}{crrrrrrrrr} 
net\# & mpbi & fsim & msim & ccan & fpon & mpon & cpon & fneg & mneg \\
\hline 0 & 12 & 0 & 0 & 54 & 1 & 0 & 8 & 2 & 0 \\
1 & 30 & 0 & 1 & 80 & 2 & 1 & 9 & 0 & 0 \\
2 & 17 & 0 & 0 & 60 & 1 & 0 & 5 & 0 & 0 \\
3 & 12 & 0 & 1 & 60 & 4 & 0 & 8 & 0 & 0 \\
4 & 3 & 1 & 0 & 43 & 1 & 0 & 7 & 0 & 0 \\
5 & 12 & 0 & 0 & 52 & 2 & 2 & 9 & 1 & 0 \\
6 & 25 & 3 & 1 & 41 & 0 & 2 & 7 & 1 & 0 \\
7 & 20 & 0 & 0 & 50 & 2 & 1 & 2 & 2 & 0 \\
8 & 8 & 1 & 0 & 44 & 1 & 0 & 5 & 1 & 0 \\
11 & 16 & 1 & 0 & 27 & 0 & 0 & 1 & 0 & 0 \\
12 & 12 & 0 & 0 & 42 & 1 & 0 & 2 & 1 & 0 \\
13 & 15 & 0 & 1 & 51 & 2 & 0 & 2 & 2 & 0 \\
14 & 15 & 0 & 3 & 28 & 1 & 0 & 3 & 2 & 1 \\
15 & 10 & 0 & 1 & 21 & 0 & 1 & 2 & 0 & 0 \\
16 & 12 & 0 & 0 & 30 & 0 & 0 & 5 & 1 & 0 \\
17 & 7 & 0 & 0 & 20 & 1 & 0 & 4 & 2 & 0 \\
18 & 7 & 0 & 0 & 34 & 0 & 0 & 4 & 5 & 0 \\
19 & 11 & 0 & 2 & 66 & 0 & 0 & 4 & 36 & 0 \\
\hline
\end{tabular}




$$
\text { mocness-1-cop220.raw WCR-82H MOCNESS tow copepods }
$$

$4 l-8$

\begin{tabular}{crrrrr} 
net\# & cneg & misc & oith & cycl & totl \\
\hline 0 & 0 & 0 & 13 & 477 & 1560 \\
1 & 0 & 4 & 11 & 336 & 1464 \\
2 & 0 & 2 & 26 & 253 & 1193 \\
3 & 0 & 1 & 30 & 251 & 1515 \\
4 & 0 & 2 & 21 & 261 & 1413 \\
5 & 0 & 1 & 24 & 281 & 1461 \\
6 & 0 & 0 & 9 & 181 & 1007 \\
7 & 0 & 0 & 32 & 262 & 1362 \\
8 & 0 & 1 & 55 & 204 & 1103 \\
11 & 0 & 1 & 30 & 120 & 819 \\
12 & 0 & 0 & 22 & 177 & 989 \\
13 & 0 & 1 & 8 & 293 & 1149 \\
14 & 0 & 4 & 33 & 186 & 947 \\
15 & 0 & 1 & 23 & 125 & 740 \\
16 & 0 & 3 & 43 & 162 & 1039 \\
17 & 0 & 2 & 31 & 149 & 905 \\
18 & 0 & 6 & 64 & 211 & 1210 \\
19 & 2 & 20 & 171 & 376 & 2103 \\
\hline
\end{tabular}


mocness-1-cop220.std WCR-82H MOCNESS tow copepods

$4 m-1$

net\# midd depi volf aliq \#ften \#mten \#cten \#fnro \#mnro

\begin{tabular}{rrrrrrrrrr}
\hline 0 & 50 & 100 & 650 & 8 & 381 & 74 & 221 & 12 & 0 \\
1 & 50 & 100 & 528 & 8 & 348 & 45 & 197 & 15 & 0 \\
2 & 50 & 100 & 493 & 8 & 292 & 130 & 341 & 0 & 0 \\
3 & 50 & 100 & 505 & 8 & 475 & 174 & 269 & 0 & 0 \\
4 & 50 & 100 & 587 & 8 & 490 & 82 & 381 & 0 & 0 \\
5 & 50 & 100 & 644 & 8 & 572 & 50 & 199 & 25 & 0 \\
6 & 50 & 100 & 599 & 8 & 214 & 0 & 214 & 0 & 0 \\
7 & 50 & 100 & 542 & 8 & 399 & 59 & 547 & 15 & 15 \\
8 & 50 & 100 & 583 & 16 & 357 & 110 & 686 & 27 & 0 \\
11 & 50 & 100 & 591 & 16 & 813 & 163 & 731 & 27 & 0 \\
12 & 50 & 100 & 562 & 16 & 541 & 85 & 683 & 28 & 0 \\
13 & 50 & 100 & 697 & 16 & 712 & 46 & 391 & 0 & 0 \\
14 & 50 & 100 & 536 & 16 & 687 & 30 & 746 & 0 & 0 \\
15 & 50 & 100 & 548 & 16 & 322 & 29 & 380 & 0 & 0 \\
16 & 50 & 100 & 561 & 16 & 599 & 29 & 542 & 0 & 0 \\
17 & 50 & 100 & 511 & 16 & 532 & 125 & 501 & 0 & 0 \\
18 & 50 & 100 & 549 & 16 & 816 & 0 & 758 & 0 & 0 \\
19 & 50 & 100 & 584 & 16 & 849 & 82 & 767 & 0 & 0 \\
\hline
\end{tabular}

net\# \#fngr \#cneo \#fund \#mund \#cund \#ccal \#fmin \#mmin \#cmin

\begin{tabular}{rrrrrrrrrr}
\hline 0 & 12 & 135 & 148 & 148 & 1095 & 74 & 258 & 123 & 972 \\
1 & 30 & 303 & 136 & 151 & 363 & 167 & 530 & 227 & 1045 \\
2 & 32 & 243 & 130 & 81 & 389 & 324 & 551 & 81 & 860 \\
3 & 0 & 412 & 253 & 301 & 222 & 301 & 887 & 317 & 760 \\
4 & 14 & 422 & 232 & 109 & 341 & 218 & 776 & 327 & 450 \\
5 & 50 & 249 & 162 & 199 & 286 & 286 & 609 & 298 & 460 \\
6 & 94 & 495 & 414 & 227 & 454 & 201 & 535 & 134 & 548 \\
7 & 44 & 517 & 89 & 89 & 89 & 295 & 842 & 458 & 872 \\
8 & 165 & 714 & 412 & 55 & 521 & 329 & 988 & 439 & 1290 \\
11 & 0 & 352 & 27 & 54 & 298 & 352 & 569 & 379 & 677 \\
12 & 28 & 512 & 57 & 28 & 455 & 228 & 740 & 541 & 854 \\
13 & 23 & 184 & 69 & 46 & 712 & 92 & 184 & 161 & 1011 \\
14 & 30 & 149 & 90 & 0 & 597 & 179 & 239 & 119 & 896 \\
15 & 88 & 555 & 146 & 263 & 58 & 234 & 351 & 146 & 409 \\
16 & 57 & 428 & 171 & 228 & 200 & 428 & 1198 & 485 & 599 \\
17 & 0 & 375 & 344 & 375 & 344 & 94 & 594 & 219 & 876 \\
18 & 0 & 495 & 350 & 175 & 845 & 525 & 554 & 495 & 1136 \\
19 & 164 & 630 & 520 & 712 & 1095 & 657 & 958 & 548 & 1643 \\
\hline
\end{tabular}


mocness-1-cop220.std WCR-82H MOCNESS tow copepods

$4 m \cdot 2$

\begin{tabular}{crrrrrrrrr} 
net\# & \#fatt & \#matt & \#catt & \#fmon & \#cmon & \#fmey & \#fcor & \#ccor & \#facr \\
\hline 0 & 37 & 0 & 135 & 62 & 0 & 0 & 12 & 0 & 591 \\
1 & 61 & 30 & 136 & 0 & 0 & 0 & 0 & 0 & 182 \\
2 & 97 & 0 & 276 & 0 & 0 & 0 & 0 & 0 & 243 \\
3 & 48 & 0 & 348 & 16 & 0 & 0 & 0 & 16 & 127 \\
4 & 27 & 0 & 341 & 0 & 0 & 0 & 0 & 0 & 82 \\
5 & 0 & 0 & 298 & 0 & 25 & 0 & 0 & 12 & 87 \\
6 & 54 & 0 & 334 & 0 & 0 & 0 & 0 & 13 & 94 \\
7 & 44 & 15 & 650 & 0 & 0 & 0 & 0 & 30 & 133 \\
8 & 27 & 0 & 714 & 0 & 0 & 27 & 0 & 0 & 247 \\
11 & 54 & 0 & 623 & 0 & 54 & 27 & 0 & 0 & 244 \\
12 & 85 & 0 & 541 & 0 & 0 & 28 & 0 & 28 & 114 \\
13 & 46 & 0 & 207 & 46 & 0 & 0 & 0 & 0 & 115 \\
14 & 60 & 30 & 119 & 0 & 149 & 30 & 0 & 0 & 478 \\
15 & 88 & 0 & 614 & 0 & 0 & 0 & 0 & 58 & 88 \\
16 & 57 & 57 & 399 & 29 & 200 & 0 & 0 & 0 & 228 \\
17 & 0 & 0 & 188 & 63 & 31 & 0 & 0 & 0 & 156 \\
18 & 0 & 29 & 262 & 0 & 29 & 58 & 0 & 0 & 525 \\
19 & 55 & 0 & 329 & 0 & 27 & 192 & 0 & 27 & 739 \\
\hline
\end{tabular}

\begin{tabular}{crrrrrrrrr} 
net\# & \#faca & \#fprv & \#farc & \#marc & \#carc & \#furc & \#faar & \#maar & \#fege \\
\hline 0 & 0 & 0 & 714 & 12 & 209 & 12 & 0 & 37 & 62 \\
1 & 30 & 0 & 712 & 91 & 182 & 15 & 0 & 15 & 76 \\
2 & 32 & 16 & 665 & 0 & 195 & 0 & 0 & 16 & 81 \\
3 & 48 & 0 & 491 & 32 & 143 & 0 & 0 & 0 & 158 \\
4 & 41 & 14 & 586 & 55 & 95 & 14 & 0 & 41 & 68 \\
5 & 0 & 0 & 572 & 25 & 174 & 12 & 0 & 0 & 137 \\
6 & 27 & 0 & 454 & 0 & 13 & 13 & 0 & 13 & 54 \\
7 & 15 & 0 & 842 & 30 & 74 & 15 & 0 & 30 & 44 \\
8 & 0 & 55 & 1784 & 27 & 467 & 82 & 0 & 27 & 137 \\
11 & 0 & 0 & 758 & 27 & 81 & 27 & 0 & 108 & 81 \\
12 & 0 & 0 & 1366 & 85 & 114 & 0 & 0 & 28 & 114 \\
13 & 23 & 0 & 368 & 0 & 138 & 0 & 0 & 184 & 138 \\
14 & 0 & 30 & 1433 & 60 & 388 & 0 & 0 & 90 & 90 \\
15 & 29 & 0 & 380 & 58 & 29 & 0 & 0 & 58 & 0 \\
16 & 0 & 57 & 570 & 0 & 29 & 0 & 29 & 0 & 285 \\
17 & 0 & 0 & 720 & 0 & 0 & 0 & 0 & 0 & 94 \\
18 & 0 & 0 & 612 & 0 & 117 & 29 & 29 & 0 & 58 \\
19 & 0 & 219 & 1972 & 137 & 657 & 219 & 0 & 0 & 82 \\
\hline
\end{tabular}


mocness-1-cop220.std WCR-82H MOCNESS tow copepods

$4 m-3$

\begin{tabular}{crrrrrrrrr} 
net \# & \#cege & \#feme & \#meme & \#cuch & \#fchi & \#mchi & \#fupl & \#mupl & \#cupl \\
\hline 0 & 160 & 0 & 0 & 62 & 0 & 0 & 0 & 0 & 0 \\
1 & 61 & 0 & 0 & 91 & 0 & 0 & 0 & 0 & 0 \\
2 & 114 & 0 & 0 & 97 & 0 & 0 & 0 & 0 & 0 \\
3 & 48 & 0 & 0 & 143 & 0 & 0 & 0 & 0 & 0 \\
4 & 82 & 0 & 0 & 191 & 0 & 0 & 0 & 0 & 0 \\
5 & 25 & 0 & 0 & 162 & 0 & 0 & 0 & 0 & 0 \\
6 & 40 & 0 & 0 & 120 & 0 & 0 & 0 & 0 & 0 \\
7 & 44 & 0 & 0 & 192 & 0 & 0 & 0 & 0 & 0 \\
8 & 110 & 0 & 0 & 274 & 0 & 0 & 0 & 0 & 0 \\
11 & 27 & 0 & 0 & 325 & 0 & 0 & 0 & 0 & 0 \\
12 & 171 & 0 & 0 & 455 & 0 & 0 & 28 & 0 & 0 \\
13 & 69 & 0 & 0 & 391 & 0 & 0 & 46 & 0 & 69 \\
14 & 60 & 0 & 0 & 418 & 0 & 0 & 30 & 0 & 149 \\
15 & 146 & 0 & 0 & 351 & 0 & 0 & 0 & 0 & 205 \\
16 & 371 & 29 & 0 & 684 & 0 & 0 & 86 & 57 & 570 \\
17 & 156 & 63 & 0 & 313 & 0 & 0 & 63 & 94 & 720 \\
18 & 146 & 0 & 0 & 525 & 0 & 0 & 233 & 29 & 583 \\
19 & 301 & 55 & 0 & 1095 & 0 & 0 & 137 & 27 & 548
\end{tabular}

net\# \#fmar \#mmar \#feuk \#meuk \#ceuk \#fpsp \#cpsp \#flop \#clop

\begin{tabular}{rrrrrrrrrr}
\hline 0 & 37 & 12 & 0 & 0 & 1587 & 0 & 37 & 12 & 0 \\
1 & 0 & 30 & 91 & 15 & 2377 & 0 & 15 & 45 & 0 \\
2 & 32 & 16 & 0 & 0 & 1897 & 0 & 32 & 16 & 0 \\
3 & 127 & 48 & 0 & 0 & 2344 & 0 & 0 & 32 & 0 \\
4 & 150 & 123 & 14 & 0 & 2071 & 14 & 55 & 27 & 0 \\
5 & 124 & 37 & 0 & 0 & 2039 & 0 & 0 & 37 & 0 \\
6 & 201 & 27 & 0 & 0 & 1510 & 27 & 40 & 27 & 0 \\
7 & 222 & 118 & 0 & 0 & 2216 & 0 & 30 & 30 & 0 \\
8 & 439 & 247 & 0 & 0 & 2360 & 0 & 0 & 110 & 0 \\
11 & 163 & 0 & 0 & 0 & 1787 & 0 & 0 & 54 & 0 \\
12 & 142 & 0 & 0 & 0 & 2276 & 0 & 28 & 57 & 0 \\
13 & 46 & 23 & 0 & 46 & 3216 & 0 & 23 & 46 & 0 \\
14 & 149 & 0 & 0 & 0 & 2716 & 0 & 60 & 30 & 0 \\
15 & 146 & 0 & 29 & 0 & 1841 & 0 & 146 & 175 & 0 \\
16 & 285 & 29 & 29 & 0 & 2566 & 0 & 86 & 86 & 114 \\
17 & 63 & 31 & 0 & 0 & 2847 & 0 & 125 & 31 & 31 \\
18 & 204 & 58 & 29 & 0 & 2710 & 29 & 29 & 29 & 87 \\
19 & 246 & 82 & 110 & 0 & 3943 & 27 & 27 & 55 & 55 \\
\hline
\end{tabular}


mocness-1-cop220.std WCR-82H MOCNESS tow copepods

$4 m \cdot 4$

\begin{tabular}{crrrrrrrrr} 
net & \#fsam & \#csam & \#fslo & \#fbra & \#mbra & \#cbra & \#fdan & \#mdan & \#cdan \\
\hline 0 & 0 & 12 & 0 & 12 & 37 & 86 & 74 & 111 & 480 \\
1 & 0 & 15 & 0 & 45 & 15 & 106 & 76 & 30 & 500 \\
2 & 0 & 16 & 0 & 65 & 16 & 114 & 32 & 0 & 503 \\
3 & 0 & 32 & 16 & 48 & 16 & 111 & 0 & 48 & 649 \\
4 & 0 & 41 & 0 & 55 & 0 & 109 & 27 & 14 & 477 \\
5 & 0 & 12 & 0 & 62 & 0 & 100 & 12 & 25 & 323 \\
6 & 0 & 27 & 0 & 27 & 0 & 54 & 54 & 13 & 80 \\
7 & 0 & 59 & 0 & 44 & 0 & 30 & 30 & 15 & 177 \\
8 & 0 & 0 & 0 & 0 & 27 & 82 & 0 & 82 & 329 \\
11 & 0 & 0 & 0 & 81 & 54 & 108 & 0 & 0 & 81 \\
12 & 0 & 28 & 0 & 85 & 57 & 228 & 0 & 28 & 199 \\
13 & 46 & 46 & 0 & 115 & 46 & 46 & 46 & 0 & 437 \\
14 & 60 & 90 & 30 & 119 & 0 & 149 & 0 & 0 & 299 \\
15 & 29 & 58 & 0 & 58 & 29 & 175 & 0 & 0 & 234 \\
16 & 114 & 86 & 57 & 114 & 57 & 171 & 29 & 0 & 0 \\
17 & 250 & 219 & 0 & 94 & 31 & 63 & 31 & 94 & 313 \\
18 & 233 & 408 & 87 & 146 & 0 & 233 & 0 & 58 & 350 \\
19 & 329 & 356 & 27 & 27 & 0 & 192 & 110 & 110 & 685 \\
\hline
\end{tabular}

\begin{tabular}{|c|c|c|c|c|c|c|c|c|c|}
\hline net\# & \#flam & \#clam & \#fvit & \#fden & \#mden & \#cden & \#ftst & \#mtst & \#ctst \\
\hline 0 & 62 & 12 & 0 & 0 & 0 & 12 & 12 & 0 & 111 \\
\hline 1 & 45 & 91 & 0 & 0 & 0 & 0 & 15 & 15 & 136 \\
\hline 2 & 32 & 65 & 0 & 0 & 0 & 16 & 0 & 16 & 97 \\
\hline 3 & 174 & 143 & 0 & 0 & 48 & 0 & 16 & 0 & 32 \\
\hline 4 & 123 & 68 & 0 & 0 & 55 & 14 & 41 & 0 & 136 \\
\hline 5 & 149 & 12 & 0 & 0 & 25 & 0 & 0 & 0 & 37 \\
\hline 6 & 54 & 0 & 0 & 27 & 0 & 0 & 0 & 13 & 54 \\
\hline 7 & 222 & 103 & 0 & 0 & 44 & 30 & 15 & 30 & 30 \\
\hline 8 & 220 & 27 & 0 & 0 & 0 & 27 & 27 & 27 & 55 \\
\hline 11 & 108 & 163 & 0 & 0 & 27 & 81 & 81 & 0 & 54 \\
\hline 12 & 341 & 85 & 0 & 28 & 28 & 0 & 0 & 0 & 28 \\
\hline 13 & 115 & 46 & 0 & 0 & 92 & 0 & 69 & 0 & 69 \\
\hline 14 & 90 & 60 & 0 & 60 & 60 & 30 & 30 & 0 & 149 \\
\hline 15 & 58 & 88 & 0 & 29 & 58 & 0 & 0 & 0 & 146 \\
\hline 16 & 228 & 29 & 0 & 143 & 57 & 114 & 29 & 0 & 57 \\
\hline 17 & 188 & 94 & 0 & 63 & 31 & 63 & 0 & 0 & 94 \\
\hline 18 & 29 & 0 & 0 & 321 & 58 & 175 & 0 & 58 & 29 \\
\hline 19 & 164 & 82 & 0 & 246 & 55 & 110 & 27 & 55 & 137 \\
\hline
\end{tabular}


mocness-1-cop220.std WCR-82H MOCNESS tow copepods

$4 m-5$

\begin{tabular}{crrrrrrrrr} 
net & \#fttu & \#mttu & \#fven & \#mven & \#fbrv & \#mbrv & \#mlon & \#fbor & \#fgra \\
\hline 0 & 0 & 0 & 0 & 0 & 0 & 0 & 0 & 0 & 0 \\
1 & 0 & 0 & 0 & 0 & 0 & 0 & 0 & 0 & 0 \\
2 & 0 & 0 & 0 & 0 & 0 & 0 & 0 & 0 & 0 \\
3 & 16 & 0 & 0 & 0 & 0 & 0 & 0 & 0 & 0 \\
4 & 0 & 14 & 0 & 0 & 0 & 0 & 0 & 0 & 14 \\
5 & 0 & 0 & 0 & 0 & 0 & 0 & 0 & 0 & 25 \\
6 & 0 & 0 & 0 & 0 & 0 & 0 & 0 & 0 & 0 \\
7 & 15 & 0 & 0 & 0 & 0 & 0 & 0 & 0 & 30 \\
8 & 27 & 0 & 0 & 0 & 0 & 0 & 0 & 0 & 165 \\
11 & 0 & 0 & 0 & 0 & 0 & 0 & 0 & 0 & 244 \\
12 & 28 & 0 & 0 & 0 & 0 & 0 & 0 & 0 & 541 \\
13 & 0 & 0 & 0 & 0 & 0 & 0 & 0 & 0 & 620 \\
14 & 30 & 0 & 0 & 0 & 0 & 0 & 0 & 0 & 896 \\
15 & 0 & 0 & 0 & 0 & 0 & 0 & 0 & 0 & 351 \\
16 & 0 & 0 & 0 & 0 & 0 & 0 & 0 & 0 & 827 \\
17 & 31 & 0 & 0 & 0 & 0 & 0 & 0 & 0 & 1001 \\
18 & 0 & 0 & 0 & 0 & 0 & 0 & 0 & 0 & 1107 \\
19 & 55 & 0 & 0 & 0 & 0 & 0 & 0 & 0 & 1506 \\
\hline
\end{tabular}

net\# \#mpsm \#fpis \#mpis \#cpsm \#fabd \#mabd \#fxip \#mxip \#cplg

\begin{tabular}{rrrrrrrrrr}
\hline 0 & 0 & 0 & 0 & 0 & 0 & 0 & 0 & 0 & 197 \\
1 & 0 & 0 & 0 & 0 & 0 & 0 & 0 & 0 & 803 \\
2 & 0 & 0 & 0 & 0 & 0 & 0 & 0 & 0 & 373 \\
3 & 0 & 0 & 0 & 0 & 0 & 0 & 0 & 0 & 301 \\
4 & 0 & 14 & 0 & 0 & 0 & 0 & 0 & 0 & 327 \\
5 & 0 & 0 & 0 & 12 & 0 & 0 & 0 & 0 & 373 \\
6 & 0 & 0 & 0 & 0 & 0 & 0 & 0 & 0 & 174 \\
7 & 15 & 0 & 0 & 15 & 0 & 0 & 0 & 0 & 0 \\
8 & 27 & 137 & 0 & 329 & 0 & 0 & 0 & 0 & 412 \\
11 & 0 & 0 & 0 & 352 & 0 & 0 & 0 & 0 & 460 \\
12 & 228 & 57 & 28 & 654 & 228 & 28 & 0 & 0 & 171 \\
13 & 299 & 207 & 138 & 781 & 115 & 0 & 0 & 0 & 620 \\
14 & 299 & 269 & 179 & 866 & 239 & 0 & 0 & 0 & 806 \\
15 & 146 & 351 & 175 & 848 & 351 & 0 & 0 & 0 & 906 \\
16 & 428 & 228 & 171 & 856 & 228 & 114 & 29 & 0 & 941 \\
17 & 438 & 344 & 94 & 1095 & 407 & 31 & 63 & 31 & 1283 \\
18 & 495 & 262 & 117 & 1632 & 262 & 87 & 146 & 29 & 962 \\
19 & 493 & 274 & 301 & 2163 & 630 & 274 & 82 & 55 & 1698 \\
\hline
\end{tabular}


mocness-1-cop220.std WCR-82H MOCNESS tow copepods

$4 m-6$

net\# \#fvio \#mvio \#cvio \#ftyp \#mtyp \#flut \#mlut \#clut \#fpap

\begin{tabular}{|c|c|c|c|c|c|c|c|c|c|}
\hline 0 & 12 & 0 & 49 & 0 & 0 & 1070 & 443 & 12 & 74 \\
\hline 1 & 61 & 45 & 30 & 0 & 0 & 1514 & 1015 & 0 & 273 \\
\hline 2 & 16 & 0 & 16 & 0 & 0 & 1265 & 973 & 16 & 65 \\
\hline 3 & 0 & 0 & 63 & 0 & 0 & 1885 & 1283 & 0 & 333 \\
\hline 4 & 27 & 0 & 0 & 0 & 0 & 1103 & 885 & 14 & 313 \\
\hline 5 & 12 & 0 & 25 & 0 & 0 & 970 & 758 & 12 & 236 \\
\hline 6 & 0 & 0 & 0 & 0 & 0 & 468 & 361 & 0 & 147 \\
\hline 7 & 30 & 15 & 15 & 0 & 0 & 1492 & 1123 & 44 & 177 \\
\hline 8 & 0 & 27 & 0 & 0 & 0 & 2141 & 1208 & 27 & 192 \\
\hline 11 & 0 & 0 & 0 & 0 & 0 & 2112 & 1571 & 0 & 163 \\
\hline 12 & 28 & 0 & 0 & 0 & 0 & 2333 & 1878 & 57 & 114 \\
\hline 13 & 0 & 0 & 46 & 0 & 0 & 1011 & 1241 & 0 & 115 \\
\hline 14 & 30 & 30 & 60 & 0 & 0 & 1702 & 925 & 30 & 179 \\
\hline 15 & 0 & 58 & 0 & 0 & 0 & 1637 & 1198 & 29 & 146 \\
\hline 16 & 0 & 0 & 29 & 0 & 0 & 1397 & 1483 & 29 & 143 \\
\hline 17 & 0 & 0 & 63 & 0 & 0 & 1846 & 1283 & 31 & 94 \\
\hline 18 & 0 & 58 & 87 & 0 & 0 & 2389 & 1166 & 58 & 0 \\
\hline 19 & 55 & 27 & 82 & 0 & 0 & 3368 & 1807 & 137 & 246 \\
\hline
\end{tabular}

net\# \#mpap \#cpap \#fspi \#mspi \#cspi \#fhal \#mhal \#chal \#augs

\begin{tabular}{rrrllrrrrr}
\hline 0 & 111 & 98 & 0 & 0 & 0 & 1353 & 37 & 148 & 0 \\
1 & 197 & 167 & 0 & 0 & 0 & 1121 & 45 & 288 & 45 \\
2 & 276 & 308 & 0 & 0 & 0 & 1022 & 65 & 341 & 130 \\
3 & 253 & 744 & 0 & 0 & 0 & 2803 & 0 & 238 & 79 \\
4 & 245 & 477 & 0 & 0 & 0 & 1567 & 95 & 232 & 82 \\
5 & 199 & 435 & 0 & 0 & 0 & 1666 & 25 & 274 & 112 \\
6 & 80 & 147 & 0 & 0 & 0 & 895 & 13 & 160 & 80 \\
7 & 89 & 369 & 0 & 0 & 30 & 798 & 0 & 295 & 44 \\
8 & 165 & 220 & 0 & 0 & 0 & 851 & 82 & 549 & 165 \\
11 & 108 & 271 & 0 & 0 & 0 & 1165 & 27 & 325 & 108 \\
12 & 142 & 370 & 0 & 0 & 0 & 1309 & 57 & 285 & 256 \\
13 & 184 & 115 & 0 & 0 & 92 & 1447 & 0 & 207 & 46 \\
14 & 149 & 239 & 0 & 0 & 0 & 896 & 30 & 209 & 119 \\
15 & 175 & 322 & 0 & 0 & 0 & 848 & 0 & 175 & 0 \\
16 & 86 & 171 & 0 & 0 & 143 & 913 & 0 & 314 & 171 \\
17 & 94 & 219 & 0 & 0 & 63 & 657 & 31 & 250 & 31 \\
18 & 146 & 291 & 0 & 0 & 29 & 437 & 0 & 437 & 58 \\
19 & 137 & 575 & 0 & 0 & 27 & 876 & 110 & 630 & 164 \\
& & & 0 & & & & & & \\
\hline
\end{tabular}


mocness-1-cop220.std WCR-82H MOCNESS tow copepods

$4 m \cdot 7$

net\# \#fhel \#mhel \#fcur \#mcur \#feth \#meth \#fpak \#mpak \#fpbi

\begin{tabular}{rrlllllrrr}
\hline 0 & 0 & 0 & 0 & 0 & 0 & 0 & 25 & 12 & 111 \\
1 & 0 & 0 & 0 & 0 & 0 & 0 & 30 & 30 & 409 \\
2 & 0 & 0 & 0 & 0 & 0 & 0 & 0 & 16 & 308 \\
3 & 0 & 0 & 0 & 0 & 0 & 0 & 0 & 16 & 285 \\
4 & 0 & 0 & 0 & 0 & 14 & 0 & 0 & 14 & 109 \\
5 & 0 & 0 & 0 & 0 & 25 & 0 & 37 & 0 & 236 \\
6 & 0 & 0 & 0 & 0 & 13 & 0 & 0 & 0 & 321 \\
7 & 0 & 0 & 0 & 0 & 0 & 0 & 0 & 0 & 74 \\
8 & 0 & 0 & 0 & 0 & 0 & 0 & 55 & 55 & 439 \\
11 & 0 & 0 & 0 & 0 & 0 & 0 & 0 & 0 & 244 \\
12 & 0 & 0 & 0 & 0 & 0 & 0 & 28 & 28 & 398 \\
13 & 0 & 0 & 0 & 0 & 0 & 0 & 23 & 0 & 414 \\
14 & 0 & 0 & 0 & 0 & 0 & 0 & 0 & 0 & 269 \\
15 & 0 & 0 & 0 & 0 & 0 & 0 & 0 & 0 & 263 \\
16 & 0 & 0 & 0 & 0 & 0 & 0 & 0 & 0 & 456 \\
17 & 0 & 0 & 0 & 31 & 0 & 0 & 0 & 0 & 188 \\
18 & 0 & 0 & 0 & 0 & 0 & 0 & 58 & 0 & 466 \\
19 & 0 & 0 & 0 & 27 & 0 & 27 & 27 & 82 & 383 \\
& & 0 & & 0 & & 0 & & & \\
\hline
\end{tabular}

net\# \#mpb: \#fsim \#msim \#ccan \#fpon \#mpon \#cpon \#fneg \#mneg

\begin{tabular}{rrrrrrrrrr}
\hline 0 & 148 & 0 & 0 & 664 & 12 & 0 & 98 & 25 & 0 \\
1 & 454 & 0 & 15 & 1211 & 30 & 15 & 136 & 0 & 0 \\
2 & 276 & 0 & 0 & 973 & 16 & 0 & 81 & 0 & 0 \\
3 & 190 & 0 & 16 & 950 & 63 & 0 & 127 & 0 & 0 \\
4 & 41 & 14 & 0 & 586 & 14 & 0 & 95 & 0 & 0 \\
5 & 149 & 0 & 0 & 647 & 25 & 25 & 112 & 12 & 0 \\
6 & 334 & 40 & 13 & 548 & 0 & 27 & 94 & 13 & 0 \\
7 & 295 & 0 & 0 & 739 & 30 & 15 & 30 & 30 & 0 \\
8 & 220 & 27 & 0 & 1208 & 27 & 0 & 137 & 27 & 0 \\
11 & 433 & 27 & 0 & 731 & 0 & 0 & 27 & 0 & 0 \\
12 & 341 & 0 & 0 & 1195 & 28 & 0 & 57 & 28 & 0 \\
13 & 345 & 0 & 23 & 1172 & 46 & 0 & 46 & 46 & 0 \\
14 & 448 & 0 & 90 & 836 & 30 & 0 & 90 & 60 & 30 \\
15 & 292 & 0 & 29 & 614 & 0 & 29 & 58 & 0 & 0 \\
16 & 342 & 0 & 0 & 856 & 0 & 0 & 143 & 29 & 0 \\
17 & 219 & 0 & 0 & 626 & 31 & 0 & 125 & 63 & 0 \\
18 & 204 & 0 & 0 & 991 & 0 & 0 & 117 & 146 & 0 \\
19 & 301 & 0 & 55 & 1807 & 0 & 0 & 110 & 986 & 0 \\
\hline
\end{tabular}




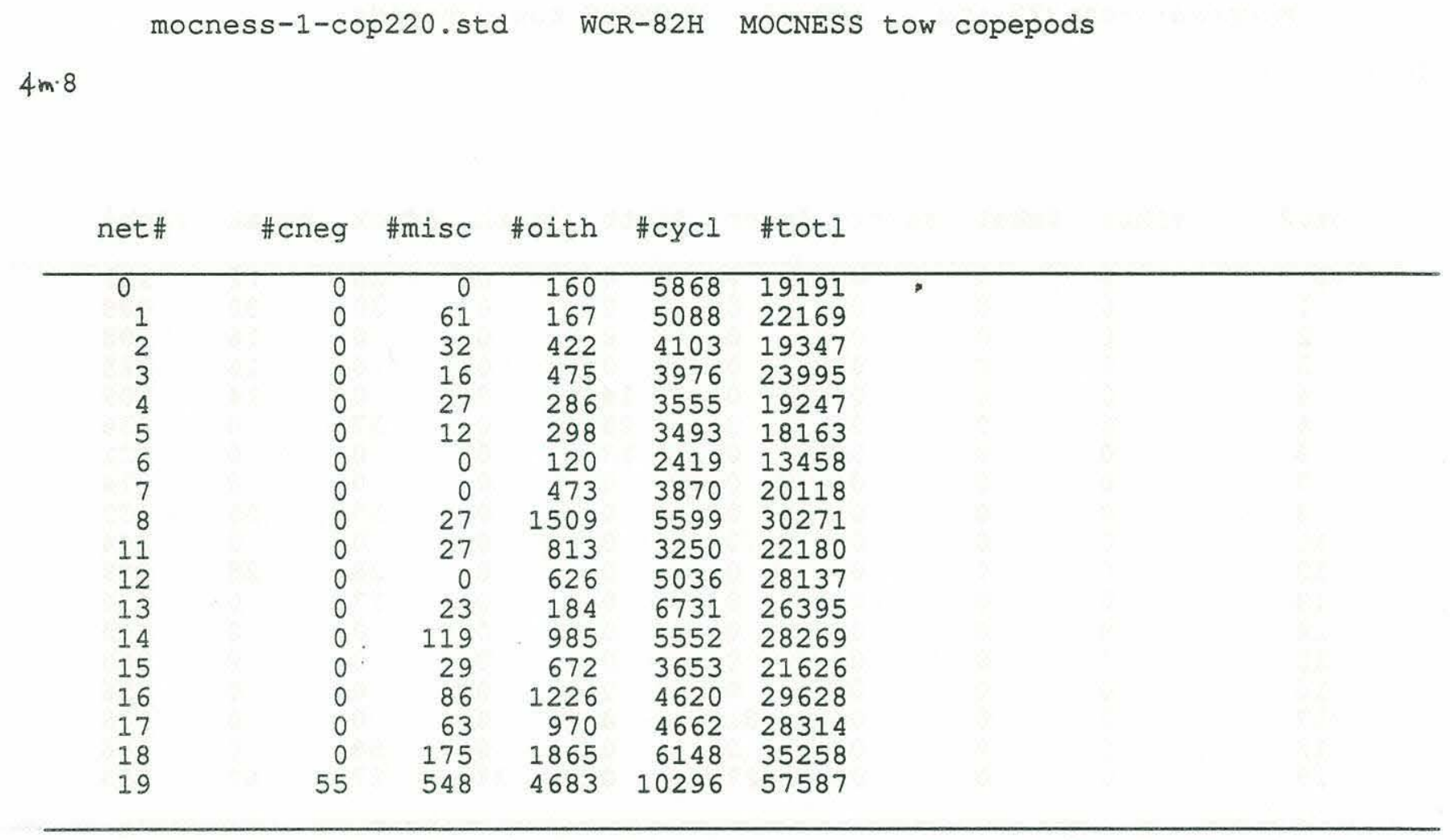


mocness-1-cop220.int WCR-82H MOCNESS tow copepods

$4 n-1$

\begin{tabular}{crrrrrrrrr} 
net\# & midd & depi & volf & aliq & iften & imten & icten & ifnro & imnro \\
\hline 0 & & & & & & & & & \\
1 & 50 & 100 & 650 & 8 & 38 & 7 & 22 & 1 & 0 \\
2 & 50 & 100 & 528 & 8 & 35 & 5 & 20 & 2 & 0 \\
3 & 50 & 100 & 505 & 8 & 29 & 13 & 34 & 0 & 0 \\
4 & 50 & 100 & 587 & 8 & 48 & 17 & 27 & 0 & 0 \\
5 & 50 & 100 & 644 & 8 & 57 & 5 & 38 & 0 & 0 \\
6 & 50 & 100 & 599 & 8 & 21 & 0 & 21 & 0 & 0 \\
7 & 50 & 100 & 542 & 8 & 40 & 6 & 55 & 1 & 1 \\
8 & 50 & 100 & 583 & 16 & 36 & 11 & 69 & 3 & 0 \\
11 & 50 & 100 & 591 & 16 & 81 & 16 & 73 & 3 & 0 \\
12 & 50 & 100 & 562 & 16 & 54 & 9 & 68 & 3 & 0 \\
13 & 50 & 100 & 697 & 16 & 71 & 5 & 39 & 0 & 0 \\
14 & 50 & 100 & 536 & 16 & 69 & 3 & 75 & 0 & 0 \\
15 & 50 & 100 & 548 & 16 & 32 & 3 & 38 & 0 & 0 \\
16 & 50 & 100 & 561 & 16 & 60 & 3 & 54 & 0 & 0 \\
17 & 50 & 100 & 511 & 16 & 53 & 13 & 50 & 0 & 0 \\
18 & 50 & 100 & 549 & 16 & 82 & 0 & 76 & 0 & 0 \\
19 & 50 & 100 & 584 & 16 & 85 & 8 & 77 & 0 & 0 \\
\hline
\end{tabular}

\begin{tabular}{crrrrrrrrr} 
net\# & ifngr & icneo & ifund & imund & icund & iccal & ifmin & immin & icmin \\
\hline 0 & 1 & 14 & 15 & 15 & 109 & 7 & 26 & 12 & 97 \\
1 & 3 & 30 & 14 & 15 & 36 & 17 & 53 & 23 & 104 \\
2 & 3 & 24 & 13 & 8 & 39 & 32 & 55 & 8 & 86 \\
3 & 0 & 41 & 25 & 30 & 22 & 30 & 89 & 32 & 76 \\
4 & 1 & 42 & 23 & 11 & 34 & 22 & 78 & 33 & 45 \\
5 & 5 & 25 & 16 & 20 & 29 & 29 & 61 & 30 & 46 \\
6 & 9 & 49 & 41 & 23 & 45 & 20 & 53 & 13 & 55 \\
7 & 4 & 52 & 9 & 9 & 9 & 30 & 84 & 46 & 87 \\
8 & 16 & 71 & 41 & 5 & 52 & 33 & 99 & 44 & 129 \\
11 & 0 & 35 & 3 & 5 & 30 & 35 & 57 & 38 & 68 \\
12 & 3 & 51 & 6 & 3 & 46 & 23 & 74 & 54 & 85 \\
13 & 2 & 18 & 7 & 5 & 71 & 9 & 18 & 16 & 101 \\
14 & 3 & 15 & 9 & 0 & 60 & 18 & 24 & 12 & 90 \\
15 & 9 & 56 & 15 & 26 & 6 & 23 & 35 & 15 & 41 \\
16 & 6 & 43 & 17 & 23 & 20 & 43 & 120 & 48 & 60 \\
17 & 0 & 38 & 34 & 38 & 34 & 9 & 59 & 22 & 88 \\
18 & 0 & 50 & 35 & 17 & 85 & 52 & 55 & 50 & 114 \\
19 & 16 & 63 & 52 & 71 & 110 & 66 & 96 & 55 & 164 \\
\hline
\end{tabular}


mocness-1-cop220.int WCR-82H MOCNESS tow copepods

$4 n-2$

\begin{tabular}{crrrrrrrrr} 
net\# & ifatt & imatt & icatt & ifmon & icmon & ifmey & ifcor & iccor & ifacr \\
\hline 0 & 4 & 0 & 14 & 6 & 0 & 0 & 1 & 0 & 59 \\
1 & 6 & 3 & 14 & 0 & 0 & 0 & 0 & 0 & 18 \\
2 & 10 & 0 & 28 & 0 & 0 & 0 & 0 & 0 & 24 \\
3 & 5 & 0 & 35 & 2 & 0 & 0 & 0 & 2 & 13 \\
4 & 3 & 0 & 34 & 0 & 0 & 0 & 0 & 0 & 8 \\
5 & 0 & 0 & 30 & 0 & 2 & 0 & 0 & 1 & 9 \\
6 & 5 & 0 & 33 & 0 & 0 & 0 & 0 & 1 & 9 \\
7 & 4 & 1 & 65 & 0 & 0 & 0 & 0 & 3 & 13 \\
8 & 3 & 0 & 71 & 0 & 0 & 3 & 0 & 0 & 25 \\
11 & 5 & 0 & 62 & 0 & 5 & 3 & 0 & 0 & 24 \\
12 & 9 & 0 & 54 & 0 & 0 & 3 & 0 & 3 & 11 \\
13 & 5 & 0 & 21 & 5 & 0 & 0 & 0 & 0 & 11 \\
14 & 6 & 3 & 12 & 0 & 15 & 3 & 0 & 0 & 48 \\
15 & 9 & 0 & 61 & 0 & 0 & 0 & 0 & 6 & 9 \\
16 & 6 & 6 & 40 & 3 & 20 & 0 & 0 & 0 & 23 \\
17 & 0 & 0 & 19 & 6 & 3 & 0 & 0 & 0 & 16 \\
18 & 0 & 3 & 26 & 0 & 3 & 6 & 0 & 0 & 52 \\
19 & 5 & 0 & 33 & 0 & 3 & 19 & 0 & 3 & 74 \\
\hline
\end{tabular}

\begin{tabular}{|c|c|c|c|c|c|c|c|c|c|}
\hline net\# & ifaca & ifprv & ifarc & imarc & icarc & ifurc & ifaar & imaar & ifege \\
\hline 0 & 0 & 0 & 71 & 1 & 21 & 1 & 0 & 4 & 6 \\
\hline 1 & 3 & 0 & 71 & 9 & 18 & 2 & 0 & 2 & 8 \\
\hline 2 & 3 & 2 & 66 & 0 & 19 & 0 & 0 & 2 & 8 \\
\hline 3 & 5 & 0 & 49 & 3 & 14 & 0 & 0 & 0 & 16 \\
\hline 4 & 4 & 1 & 59 & 5 & 10 & 1 & 0 & 4 & 7 \\
\hline 5 & 0 & 0 & 57 & 2 & 17 & 1 & 0 & 0 & 14 \\
\hline 6 & 3 & 0 & 45 & 0 & 1 & 1 & 0 & 1 & 5 \\
\hline 7 & 1 & 0 & 84 & 3 & 7 & 1 & 0 & 3 & 4 \\
\hline 8 & 0 & 5 & 178 & 3 & 47 & 8 & 0 & 3 & 14 \\
\hline 11 & 0 & 0 & 76 & 3 & 8 & 3 & 0 & 11 & 8 \\
\hline 12 & 0 & 0 & 137 & 9 & 11 & 0 & 0 & 3 & 11 \\
\hline 13 & 2 & 0 & 37 & 0 & 14 & 0 & 0 & 18 & 14 \\
\hline 14 & 0 & 3 & 143 & 6 & 39 & 0 & 0 & 9 & 9 \\
\hline 15 & 3 & 0 & 38 & 6 & 3 & 0 & 0 & 6 & 0 \\
\hline 16 & 0 & 6 & 57 & 0 & 3 & 0 & 3 & 0 & 29 \\
\hline 17 & 0 & 0 & 72 & 0 & 0 & 0 & 0 & 0 & 9 \\
\hline 18 & 0 & 0 & 61 & 0 & 12 & 3 & 3 & 0 & 6 \\
\hline 19 & 0 & 22 & 197 & 14 & 66 & 22 & 0 & 0 & 8 \\
\hline
\end{tabular}


mocness-1-cop220.int WCR-82H MOCNESS tow copepods

$4 n \cdot 3$

net\# icege ifeme imeme icuch ifchi imchi ifupl imupl icupl

\begin{tabular}{rrllllllll}
\hline 0 & 16 & 0 & 0 & 6 & 0 & 0 & 0 & 0 & 0 \\
1 & 6 & 0 & 0 & 9 & 0 & 0 & 0 & 0 & 0 \\
2 & 11 & 0 & 0 & 10 & 0 & 0 & 0 & 0 & 0 \\
3 & 5 & 0 & 0 & 14 & 0 & 0 & 0 & 0 & 0 \\
4 & 8 & 0 & 0 & 19 & 0 & 0 & 0 & 0 & 0 \\
5 & 2 & 0 & 0 & 16 & 0 & 0 & 0 & 0 & 0 \\
6 & 4 & 0 & 0 & 12 & 0 & 0 & 0 & 0 & 0 \\
7 & 4 & 0 & 0 & 19 & 0 & 0 & 0 & 0 & 0 \\
8 & 11 & 0 & 0 & 27 & 0 & 0 & 0 & 0 & 0 \\
11 & 3 & 0 & 0 & 33 & 0 & 0 & 0 & 0 & 0 \\
12 & 17 & 0 & 0 & 46 & 0 & 0 & 3 & 0 & 0 \\
13 & 6 & 0 & 0 & 39 & 0 & 0 & 5 & 0 & 7 \\
14 & 15 & 0 & 0 & 42 & 0 & 0 & 3 & 0 & 15 \\
15 & 37 & 3 & 0 & 35 & 0 & 0 & 0 & 0 & 20 \\
16 & 16 & 6 & 0 & 68 & 0 & 0 & 9 & 6 & 57 \\
17 & 15 & 0 & 0 & 31 & 0 & 0 & 6 & 9 & 72 \\
18 & 30 & 5 & 0 & 110 & 0 & 0 & 23 & 3 & 58 \\
19 & & & 0 & & 0 & 0 & 14 & 3 & 55 \\
\hline
\end{tabular}

\begin{tabular}{rrrrrrrrrr} 
net\# & ifmar & immar & ifeuk & imeuk & iceuk & ifpsp & icpsp & iflop & iclop \\
\hline 0 & 4 & 1 & 0 & 0 & 159 & 0 & 4 & 1 & 0 \\
1 & 0 & 3 & 9 & 2 & 238 & 0 & 2 & 5 & 0 \\
2 & 3 & 2 & 0 & 0 & 190 & 0 & 3 & 2 & 0 \\
3 & 13 & 5 & 0 & 0 & 234 & 0 & 0 & 3 & 0 \\
4 & 15 & 12 & 1 & 0 & 207 & 1 & 5 & 3 & 0 \\
5 & 12 & 4 & 0 & 0 & 204 & 0 & 0 & 4 & 0 \\
6 & 20 & 3 & 0 & 0 & 151 & 3 & 4 & 3 & 0 \\
7 & 22 & 12 & 0 & 0 & 222 & 0 & 3 & 3 & 0 \\
8 & 44 & 25 & 0 & 0 & 236 & 0 & 0 & 11 & 0 \\
11 & 16 & 0 & 0 & 0 & 179 & 0 & 0 & 5 & 0 \\
12 & 14 & 0 & 0 & 0 & 228 & 0 & 3 & 6 & 0 \\
13 & 5 & 2 & 0 & 5 & 322 & 0 & 2 & 5 & 0 \\
14 & 15 & 0 & 0 & 0 & 272 & 0 & 6 & 3 & 0 \\
15 & 15 & 0 & 3 & 0 & 184 & 0 & 15 & 18 & 0 \\
16 & 29 & 3 & 3 & 0 & 257 & 0 & 9 & 9 & 11 \\
17 & 6 & 3 & 0 & 0 & 285 & 0 & 13 & 3 & 3 \\
18 & 20 & 6 & 3 & 0 & 271 & 3 & 3 & 3 & 9 \\
19 & 25 & 8 & 11 & 0 & 394 & 3 & 3 & 5 & 5 \\
\hline
\end{tabular}


mocness-1-cop220.int WCR-82H MOCNESS tow copepods

$4 n-4$

\begin{tabular}{crrrrrrrrr} 
net\# & ifsam & icsam & ifslo & ifbra & imbra & icbra & ifdan & imdan & icdan \\
\hline 0 & 0 & 1 & 0 & 1 & 4 & 9 & 7 & 11 & 48 \\
1 & 0 & 2 & 0 & 5 & 2 & 11 & 8 & 3 & 50 \\
2 & 0 & 2 & 0 & 6 & 2 & 11 & 3 & 0 & 50 \\
3 & 0 & 3 & 2 & 5 & 2 & 11 & 0 & 5 & 65 \\
4 & 0 & 4 & 0 & 5 & 0 & 11 & 3 & 1 & 48 \\
5 & 0 & 1 & 0 & 6 & 0 & 10 & 1 & 2 & 32 \\
6 & 0 & 3 & 0 & 3 & 0 & 5 & 5 & 1 & 8 \\
7 & 0 & 6 & 0 & 4 & 0 & 3 & 3 & 1 & 18 \\
8 & 0 & 0 & 0 & 0 & 3 & 8 & 0 & 8 & 33 \\
11 & 0 & 0 & 0 & 8 & 5 & 11 & 0 & 0 & 8 \\
12 & 0 & 3 & 0 & 9 & 6 & 23 & 0 & 3 & 20 \\
13 & 5 & 5 & 0 & 11 & 5 & 5 & 5 & 0 & 44 \\
14 & 6 & 9 & 3 & 12 & 0 & 15 & 0 & 0 & 30 \\
15 & 3 & 6 & 0 & 6 & 3 & 18 & 0 & 0 & 23 \\
16 & 11 & 9 & 6 & 11 & 6 & 17 & 3 & 0 & 0 \\
17 & 25 & 22 & 0 & 9 & 3 & 6 & 3 & 9 & 31 \\
18 & 23 & 41 & 9 & 15 & 0 & 23 & 0 & 6 & 35 \\
19 & 33 & 36 & 3 & 3 & 0 & 19 & 11 & 11 & 68 \\
\hline
\end{tabular}

\begin{tabular}{crrrrrrrrr} 
net\# & iflam & iclam & ifvit & ifden & imden & icden & iftst & imtst & ictst \\
\hline 0 & & & & & & & & & \\
1 & 5 & 9 & 0 & 0 & 0 & 1 & 1 & 0 & 11 \\
2 & 3 & 6 & 0 & 0 & 0 & 0 & 2 & 2 & 14 \\
3 & 17 & 14 & 0 & 0 & 0 & 2 & 0 & 2 & 10 \\
4 & 12 & 7 & 0 & 0 & 5 & 0 & 2 & 0 & 3 \\
5 & 15 & 1 & 0 & 0 & 2 & 0 & 4 & 0 & 14 \\
6 & 5 & 0 & 0 & 3 & 0 & 0 & 0 & 0 & 4 \\
7 & 22 & 10 & 0 & 0 & 4 & 3 & 1 & 3 & 3 \\
8 & 22 & 3 & 0 & 0 & 0 & 3 & 3 & 3 & 5 \\
11 & 11 & 16 & 0 & 0 & 3 & 8 & 8 & 0 & 5 \\
12 & 34 & 9 & 0 & 3 & 3 & 0 & 0 & 0 & 3 \\
13 & 11 & 5 & 0 & 0 & 9 & 0 & 7 & 0 & 7 \\
14 & 9 & 6 & 0 & 6 & 6 & 3 & 3 & 0 & 15 \\
15 & 6 & 9 & 0 & 3 & 6 & 0 & 0 & 0 & 15 \\
16 & 23 & 3 & 0 & 14 & 6 & 11 & 3 & 0 & 6 \\
17 & 19 & 9 & 0 & 6 & 3 & 6 & 0 & 0 & 9 \\
18 & 3 & 0 & 0 & 32 & 6 & 17 & 0 & 6 & 3 \\
19 & 16 & 8 & 0 & 25 & 5 & 11 & 3 & 5 & 14 \\
\hline
\end{tabular}


mocness-1-cop220.int WCR-82H MOCNESS tow copepods

$4 n-5$

\begin{tabular}{cccccccccc} 
net\# & ifttu & imttu & ifven & imven & ifbrv & imbrv & imlon & ifbor & ifgra \\
\hline 0 & 0 & 0 & 0 & 0 & 0 & 0 & 0 & 0 & 0 \\
1 & 0 & 0 & 0 & 0 & 0 & 0 & 0 & 0 & 0 \\
2 & 0 & 0 & 0 & 0 & 0 & 0 & 0 & 0 & 0 \\
3 & 2 & 0 & 0 & 0 & 0 & 0 & 0 & 0 & 0 \\
4 & 0 & 1 & 0 & 0 & 0 & 0 & 0 & 0 & 1 \\
5 & 0 & 0 & 0 & 0 & 0 & 0 & 0 & 0 & 2 \\
6 & 0 & 0 & 0 & 0 & 0 & 0 & 0 & 0 & 0 \\
7 & 1 & 0 & 0 & 0 & 0 & 0 & 0 & 0 & 3 \\
8 & 3 & 0 & 0 & 0 & 0 & 0 & 0 & 0 & 16 \\
11 & 0 & 0 & 0 & 0 & 0 & 0 & 0 & 0 & 24 \\
12 & 3 & 0 & 0 & 0 & 0 & 0 & 0 & 0 & 54 \\
13 & 0 & 0 & 0 & 0 & 0 & 0 & 0 & 0 & 62 \\
14 & 3 & 0 & 0 & 0 & 0 & 0 & 0 & 0 & 90 \\
15 & 0 & 0 & 0 & 0 & 0 & 0 & 0 & 0 & 35 \\
16 & 0 & 0 & 0 & 0 & 0 & 0 & 0 & 0 & 83 \\
17 & 3 & 0 & 0 & 0 & 0 & 0 & 0 & 0 & 100 \\
18 & 0 & 0 & 0 & 0 & 0 & 0 & 0 & 0 & 111 \\
19 & 5 & 0 & 0 & 0 & 0 & 0 & 0 & 0 & 151 \\
\hline
\end{tabular}

net\# impsm ifpis impis icpsm ifabd imabd ifxip imxip icplg

\begin{tabular}{rrrrrrrrrr}
\hline 0 & 0 & 0 & 0 & 0 & 0 & 0 & 0 & 0 & 20 \\
1 & 0 & 0 & 0 & 0 & 0 & 0 & 0 & 0 & 80 \\
2 & 0 & 0 & 0 & 0 & 0 & 0 & 0 & 0 & 37 \\
3 & 0 & 0 & 0 & 0 & 0 & 0 & 0 & 0 & 30 \\
4 & 0 & 1 & 0 & 0 & 0 & 0 & 0 & 0 & 33 \\
5 & 0 & 0 & 0 & 1 & 0 & 0 & 0 & 0 & 37 \\
6 & 0 & 0 & 0 & 0 & 0 & 0 & 0 & 0 & 17 \\
7 & 1 & 0 & 0 & 1 & 0 & 0 & 0 & 0 & 0 \\
8 & 3 & 14 & 0 & 33 & 0 & 0 & 0 & 0 & 41 \\
11 & 0 & 0 & 0 & 35 & 0 & 0 & 0 & 0 & 46 \\
12 & 23 & 6 & 3 & 65 & 23 & 3 & 0 & 0 & 17 \\
13 & 30 & 21 & 14 & 78 & 11 & 0 & 0 & 0 & 62 \\
14 & 30 & 27 & 18 & 87 & 24 & 0 & 0 & 0 & 81 \\
15 & 15 & 35 & 18 & 85 & 35 & 0 & 0 & 0 & 91 \\
16 & 43 & 23 & 17 & 86 & 23 & 11 & 3 & 0 & 94 \\
17 & 44 & 34 & 9 & 110 & 41 & 3 & 6 & 3 & 128 \\
18 & 50 & 26 & 12 & 163 & 26 & 9 & 15 & 3 & 96 \\
19 & 49 & 27 & 30 & 216 & 63 & 27 & 8 & 5 & 170 \\
\hline
\end{tabular}


mocness-1-cop220.int WCR-82H MOCNESS tow copepods

$4 n-6$

net\# ifvio imvio icvio iftyp imtyp iflut imlut iclut ifpap

\begin{tabular}{rrllllrrrr}
\hline 0 & 1 & 0 & 5 & 0 & 0 & 107 & 44 & 1 & 7 \\
1 & 6 & 5 & 3 & 0 & 0 & 151 & 101 & 0 & 27 \\
2 & 2 & 0 & 2 & 0 & 0 & 127 & 97 & 2 & 6 \\
3 & 0 & 0 & 6 & 0 & 0 & 188 & 128 & 0 & 33 \\
4 & 3 & 0 & 0 & 0 & 0 & 110 & 89 & 1 & 31 \\
5 & 1 & 0 & 2 & 0 & 0 & 97 & 76 & 1 & 24 \\
6 & 0 & 0 & 0 & 0 & 0 & 47 & 36 & 0 & 15 \\
7 & 3 & 1 & 1 & 0 & 0 & 149 & 112 & 4 & 18 \\
8 & 0 & 3 & 0 & 0 & 0 & 214 & 121 & 3 & 19 \\
11 & 0 & 0 & 0 & 0 & 0 & 211 & 157 & 0 & 16 \\
12 & 3 & 0 & 0 & 0 & 0 & 233 & 188 & 6 & 11 \\
13 & 0 & 0 & 5 & 0 & 0 & 101 & 124 & 0 & 11 \\
14 & 3 & 3 & 6 & 0 & 0 & 170 & 93 & 3 & 18 \\
15 & 0 & 6 & 0 & 0 & 0 & 164 & 120 & 3 & 15 \\
16 & 0 & 0 & 3 & 0 & 0 & 140 & 148 & 3 & 14 \\
17 & 0 & 0 & 6 & 0 & 0 & 185 & 128 & 3 & 9 \\
18 & 0 & 6 & 9 & 0 & 0 & 239 & 117 & 6 & 0 \\
19 & 5 & 3 & 8 & 0 & 0 & 337 & 181 & 14 & 25 \\
\hline
\end{tabular}

net\# impap icpap ifspi imspi icspi ifhal imhal ichal iaugs

\begin{tabular}{rrlllllllr}
\hline 0 & 11 & 10 & 0 & 0 & 0 & 135 & 4 & 15 & 0 \\
1 & 20 & 17 & 0 & 0 & 0 & 112 & 5 & 29 & 5 \\
2 & 28 & 31 & 0 & 0 & 0 & 102 & 6 & 34 & 13 \\
3 & 25 & 74 & 0 & 0 & 0 & 280 & 0 & 24 & 8 \\
4 & 25 & 48 & 0 & 0 & 0 & 157 & 10 & 23 & 8 \\
5 & 20 & 44 & 0 & 0 & 0 & 167 & 2 & 27 & 11 \\
6 & 8 & 15 & 0 & 0 & 0 & 90 & 1 & 16 & 8 \\
7 & 9 & 37 & 0 & 0 & 3 & 80 & 0 & 30 & 4 \\
8 & 16 & 22 & 0 & 0 & 0 & 85 & 8 & 55 & 16 \\
11 & 11 & 27 & 0 & 0 & 0 & 116 & 3 & 33 & 11 \\
12 & 14 & 37 & 0 & 0 & 0 & 131 & 6 & 28 & 26 \\
13 & 18 & 11 & 0 & 0 & 9 & 145 & 0 & 21 & 5 \\
14 & 15 & 24 & 0 & 0 & 0 & 90 & 3 & 21 & 12 \\
15 & 18 & 32 & 0 & 0 & 0 & 85 & 0 & 18 & 0 \\
16 & 9 & 17 & 0 & 0 & 14 & 91 & 0 & 31 & 17 \\
17 & 9 & 22 & 0 & 0 & 6 & 66 & 3 & 25 & 3 \\
18 & 15 & 29 & 0 & 0 & 3 & 44 & 0 & 44 & 6 \\
19 & 14 & 58 & 0 & 0 & 3 & 88 & 11 & 63 & 16 \\
\hline
\end{tabular}


mocness-1-cop220.int WCR-82H MOCNESS tow copepods

$4 n-7$

\begin{tabular}{|c|c|c|c|c|c|c|c|c|c|}
\hline net\# & ifhel & imhel & ifcur & imcur & ifeth & imeth & ifpak & impak & ifpbi \\
\hline 0 & 0 & 0 & 0 & 0 & 0 & 0 & 2 & 1 & 11 \\
\hline 1 & 0 & 0 & 0 & 0 & 0 & 0 & 3 & 3 & 41 \\
\hline 2 & 0 & 0 & 0 & 0 & 0 & 0 & 0 & 2 & 31 \\
\hline 3 & 0 & 0 & 0 & 0 & 0 & 0 & 0 & 2 & 29 \\
\hline 4 & 0 & 0 & 0 & 0 & 1 & 0 & 0 & 1 & 11 \\
\hline 5 & 0 & 0 & 0 & 0 & 2 & 0 & 4 & 0 & 24 \\
\hline 6 & 0 & 0 & 0 & 0 & 1 & 0 & 0 & 0 & 32 \\
\hline 7 & 0 & 0 & 0 & 0 & 0 & 0 & 0 & 0 & 7 \\
\hline 8 & 0 & 0 & 0 & 0 & 0 & 0 & 5 & 5 & 44 \\
\hline 11 & 0 & 0 & 0 & 0 & 0 & 0 & 0 & 0 & 24 \\
\hline 12 & 0 & 0 & 0 & 0 & 0 & 0 & 3 & 3 & 40 \\
\hline 13 & 0 & 0 & 0 & 0 & 0 & 0 & 2 & 0 & 41 \\
\hline 14 & 0 & 0 & 0 & 0 & 0 & 0 & 0 & 0 & 27 \\
\hline 15 & 0 & 0 & 0 & 0 & 0 & 0 & 0 & 0 & 26 \\
\hline 16 & 0 & 0 & 0 & 0 & 0 & 0 & 0 & 0 & 46 \\
\hline 17 & 0 & 0 & 0 & 3 & 0 & 0 & 0 & 0 & 19 \\
\hline 18 & 0 & 0 & 0 & 0 & 0 & 0 & 6 & 0 & 47 \\
\hline 19 & 0 & 0 & 0 & 3 & 0 & 3 & 3 & 8 & 38 \\
\hline
\end{tabular}

\begin{tabular}{crrrrrrrrr} 
net\# & impbi & ifsim & imsim & iccan & ifpon & impon & icpon & ifneg & imneg \\
\hline 0 & 15 & 0 & 0 & 66 & 1 & 0 & 10 & 2 & 0 \\
1 & 45 & 0 & 2 & 121 & 3 & 2 & 14 & 0 & 0 \\
2 & 28 & 0 & 0 & 97 & 2 & 0 & 8 & 0 & 0 \\
3 & 19 & 0 & 2 & 95 & 6 & 0 & 13 & 0 & 0 \\
4 & 4 & 1 & 0 & 59 & 1 & 0 & 10 & 0 & 0 \\
5 & 15 & 0 & 0 & 65 & 2 & 2 & 11 & 1 & 0 \\
6 & 33 & 4 & 1 & 55 & 0 & 3 & 9 & 1 & 0 \\
7 & 30 & 0 & 0 & 74 & 3 & 1 & 3 & 3 & 0 \\
8 & 22 & 3 & 0 & 121 & 3 & 0 & 14 & 3 & 0 \\
11 & 43 & 3 & 0 & 73 & 0 & 0 & 3 & 0 & 0 \\
12 & 34 & 0 & 0 & 119 & 3 & 0 & 6 & 3 & 0 \\
13 & 34 & 0 & 2 & 117 & 5 & 0 & 5 & 5 & 0 \\
14 & 45 & 0 & 9 & 84 & 3 & 0 & 9 & 6 & 3 \\
15 & 29 & 0 & 3 & 61 & 0 & 3 & 6 & 0 & 0 \\
16 & 34 & 0 & 0 & 86 & 0 & 0 & 14 & 3 & 0 \\
17 & 22 & 0 & 0 & 63 & 3 & 0 & 13 & 6 & 0 \\
18 & 20 & 0 & 0 & 99 & 0 & 0 & 12 & 15 & 0 \\
19 & 30 & 0 & 5 & 181 & 0 & 0 & 11 & 99 & 0 \\
\hline
\end{tabular}


mocness-1-cop220.int WCR-82H MOCNESS tow copepods

$4 n-8$

net\# icneg imisc ioith icycl itotl

\begin{tabular}{|c|c|c|c|c|c|}
\hline $\begin{array}{r}0 \\
1 \\
2 \\
3 \\
4 \\
5 \\
6 \\
7 \\
8 \\
11 \\
12 \\
13 \\
14 \\
15 \\
16 \\
17 \\
18 \\
19\end{array}$ & $\begin{array}{l}0 \\
0 \\
0 \\
0 \\
0 \\
0 \\
0 \\
0 \\
0 \\
0 \\
0 \\
0 \\
0 \\
0 \\
0 \\
0 \\
0 \\
5\end{array}$ & $\begin{array}{r}0 \\
6 \\
3 \\
2 \\
3 \\
1 \\
0 \\
0 \\
3 \\
3 \\
0 \\
2 \\
12 \\
3 \\
9 \\
6 \\
17 \\
55\end{array}$ & $\begin{array}{r}16 \\
17 \\
42 \\
48 \\
29 \\
30 \\
12 \\
47 \\
151 \\
81 \\
63 \\
18 \\
99 \\
67 \\
123 \\
97 \\
186 \\
468\end{array}$ & $\begin{array}{r}587 \\
509 \\
410 \\
398 \\
356 \\
349 \\
242 \\
387 \\
560 \\
325 \\
504 \\
673 \\
555 \\
365 \\
462 \\
466 \\
615 \\
1030\end{array}$ & $\begin{array}{l}1919 \\
2217 \\
1935 \\
2400 \\
1925 \\
1816 \\
1346 \\
2012 \\
3027 \\
2218 \\
2814 \\
2639 \\
2827 \\
2163 \\
2963 \\
2831 \\
3526 \\
5759\end{array}$ \\
\hline
\end{tabular}


mocness-1-cop222. raw WCR-82H MOCNESS tow copepods

$40-1$

\begin{tabular}{rrrrrrrrrr} 
net\# & midd & depi & volf & aliq & ften & mten & cten & fnro & mnro \\
\hline 18 & 5 & 10 & 244 & 8 & 0 & 0 & 0 & 10 & 0 \\
17 & 15 & 10 & 177 & 8 & 0 & 0 & 0 & 1 & 0 \\
16 & 25 & 10 & 185 & 8 & 0 & 0 & 0 & 5 & 0 \\
15 & 35 & 10 & 227 & 8 & 1 & 0 & 2 & 4 & 0 \\
14 & 45 & 10 & 265 & 8 & 4 & 0 & 8 & 3 & 0 \\
13 & 55 & 10 & 220 & 8 & 2 & 0 & 3 & 0 & 0 \\
12 & 65 & 10 & 231 & 8 & 81 & 1 & 34 & 10 & 0 \\
11 & 75 & 10 & 339 & 8 & 114 & 6 & 82 & 3 & 0 \\
8 & 85 & 10 & 218 & 4 & 20 & 26 & 42 & 2 & 0 \\
7 & 95 & 10 & 225 & 4 & 3 & 6 & 3 & 1 & 0 \\
6 & 105 & 10 & 217 & 2 & 0 & 12 & 2 & 0 & 0 \\
5 & 115 & 10 & 167 & 1 & 0 & 21 & 1 & 2 & 0 \\
4 & 125 & 10 & 282 & 1 & 3 & 14 & 4 & 0 & 0 \\
3 & 135 & 10 & 284 & 1 & 0 & 5 & 0 & 3 & 0 \\
2 & 145 & 10 & 221 & 1 & 0 & 4 & 1 & 1 & 0 \\
1 & 155 & 10 & 181 & 1 & 1 & 3 & 3 & 0 & 1 \\
\hline
\end{tabular}

\begin{tabular}{rrrrrrrrrr} 
net\# & cneo & fund & mund & cund & ccal & fmin & mmin & cmin & felg \\
\hline 18 & 1 & 39 & 29 & 40 & 13 & 83 & 43 & 21 & 0 \\
17 & 14 & 8 & 11 & 57 & 47 & 57 & 36 & 37 & 0 \\
16 & 16 & 3 & 7 & 99 & 36 & 52 & 32 & 105 & 0 \\
15 & 55 & 4 & 2 & 53 & 31 & 45 & 47 & 109 & 0 \\
14 & 42 & 5 & 7 & 18 & 94 & 79 & 59 & 106 & 0 \\
13 & 20 & 7 & 2 & 2 & 31 & 31 & 23 & 26 & 0 \\
12 & 23 & 9 & 6 & 0 & 33 & 28 & 21 & 15 & 0 \\
11 & 25 & 6 & 1 & 0 & 40 & 24 & 9 & 15 & 0 \\
8 & 5 & 0 & 0 & 0 & 7 & 1 & 0 & 4 & 0 \\
7 & 3 & 0 & 0 & 0 & 5 & 1 & 0 & 0 & 0 \\
6 & 5 & 0 & 0 & 0 & 2 & 0 & 0 & 0 & 0 \\
5 & 11 & 0 & 0 & 0 & 13 & 0 & 0 & 0 & 0 \\
4 & 6 & 0 & 0 & 1 & 24 & 0 & 0 & 0 & 0 \\
3 & 10 & 0 & 0 & 1 & 32 & 0 & 0 & 0 & 0 \\
2 & 11 & 0 & 0 & 1 & 6 & 0 & 1 & 1 & 0 \\
1 & 5 & 1 & 0 & 2 & 26 & 2 & 0 & 3 & 0 \\
\hline
\end{tabular}




$$
\text { mocness-1-cop222. raw WCR-82H MOCNESS tow copepods }
$$

$40-2$

\begin{tabular}{rrrrrrrrrr} 
net\# & celg & fatt & matt & catt & fmey & facr & fpav & farc & marc \\
\hline 18 & 0 & 1 & 0 & 7 & 0 & 16 & 11 & 26 & 0 \\
17 & 0 & 5 & 1 & 9 & 0 & 37 & 10 & 17 & 4 \\
16 & 0 & 6 & 0 & 21 & 0 & 0 & 3 & 27 & 1 \\
15 & 0 & 2 & 2 & 15 & 0 & 5 & 3 & 45 & 4 \\
14 & 0 & 2 & 0 & 39 & 0 & 13 & 1 & 104 & 2 \\
13 & 0 & 3 & 5 & 14 & 5 & 0 & 0 & 47 & 2 \\
12 & 0 & 4 & 5 & 26 & 6 & 0 & 3 & 66 & 2 \\
11 & 0 & 3 & 1 & 17 & 5 & 0 & 3 & 162 & 4 \\
8 & 0 & 1 & 0 & 1 & 0 & 0 & 27 & 51 & 0 \\
7 & 0 & 1 & 0 & 0 & 0 & 0 & 0 & 3 & 0 \\
6 & 0 & 0 & 0 & 0 & 0 & 0 & 1 & 9 & 0 \\
5 & 0 & 0 & 0 & 0 & 0 & 0 & 0 & 8 & 0 \\
4 & 0 & 2 & 0 & 0 & 0 & 0 & 1 & 19 & 0 \\
3 & 1 & 0 & 0 & 1 & 1 & 0 & 0 & 16 & 0 \\
2 & 0 & 0 & 0 & 0 & 0 & 0 & 1 & 10 & 0 \\
1 & 0 & 0 & 0 & 0 & 1 & 0 & 0 & 4 & 0 \\
\hline
\end{tabular}

\begin{tabular}{rrrrrrrrrr} 
net\# & carc & furc & faar & mar & fege & cege & fgmi & cgmi & feme \\
\hline 18 & 13 & 18 & 0 & 0 & 0 & 0 & 0 & 0 & 0 \\
17 & 22 & 10 & 0 & 0 & 0 & 0 & 0 & 0 & 1 \\
16 & 12 & 4 & 0 & 0 & 0 & 0 & 0 & 0 & 5 \\
15 & 12 & 1 & 0 & 0 & 0 & 0 & 0 & 0 & 4 \\
14 & 3 & 0 & 0 & 0 & 0 & 0 & 0 & 0 & 2 \\
13 & 4 & 1 & 0 & 0 & 0 & 0 & 0 & 0 & 0 \\
12 & 2 & 1 & 0 & 0 & 8 & 2 & 1 & 1 & 2 \\
11 & 4 & 0 & 0 & 1 & 37 & 44 & 7 & 4 & 1 \\
8 & 12 & 5 & 1 & 6 & 10 & 111 & 15 & 16 & 0 \\
7 & 0 & 2 & 1 & 6 & 4 & 5 & 2 & 9 & 0 \\
6 & 0 & 1 & 0 & 3 & 1 & 8 & 8 & 23 & 0 \\
5 & 4 & 2 & 0 & 1 & 3 & 2 & 9 & 58 & 0 \\
4 & 0 & 2 & 3 & 0 & 3 & 2 & 15 & 176 & 0 \\
3 & 0 & 0 & 8 & 0 & 5 & 1 & 4 & 97 & 0 \\
2 & 0 & 1 & 0 & 11 & 1 & 0 & 3 & 58 & 0 \\
1 & 0 & 20 & 0 & 7 & 1 & 0 & 2 & 89 & 1 \\
\hline
\end{tabular}


mocness-1-cop222.raw WCR-82H MOCNESS tow copepods

$4 o-3$

\begin{tabular}{rrrrrrrrrr} 
net\# & meme & fros & cuch & fchi & mchi & fupl & mupl & feuk & meuk \\
\hline 18 & 0 & 0 & 0 & 0 & 0 & 5 & 0 & 17 & 12 \\
17 & 0 & 0 & 1 & 0 & 0 & 7 & 0 & 15 & 2 \\
16 & 2 & 0 & 11 & 0 & 0 & 1 & 0 & 4 & 4 \\
15 & 0 & 0 & 30 & 0 & 0 & 8 & 0 & 4 & 4 \\
14 & 2 & 0 & 267 & 0 & 0 & 4 & 0 & 7 & 3 \\
13 & 0 & 0 & 144 & 0 & 0 & 3 & 0 & 5 & 1 \\
12 & 1 & 0 & 27 & 1 & 0 & 2 & 0 & 11 & 4 \\
11 & 1 & 0 & 5 & 3 & 0 & 3 & 0 & 10 & 2 \\
8 & 0 & 0 & 5 & 3 & 0 & 2 & 0 & 6 & 4 \\
7 & 0 & 1 & 1 & 1 & 1 & 3 & 0 & 4 & 0 \\
6 & 0 & 0 & 1 & 2 & 5 & 2 & 0 & 1 & 0 \\
5 & 0 & 0 & 10 & 0 & 5 & 0 & 0 & 5 & 0 \\
4 & 0 & 0 & 18 & 2 & 3 & 3 & 0 & 2 & 1 \\
3 & 0 & 0 & 0 & 2 & 2 & 2 & 0 & 1 & 0 \\
2 & 0 & 0 & 26 & 1 & 0 & 0 & 0 & 3 & 0 \\
1 & 0 & 0 & -999 & 0 & 0 & 1 & 0 & 1 & 1 \\
\hline
\end{tabular}

\begin{tabular}{rrrrrrrrrr} 
net\# & ceuk & fpsp & cpsp & fsco & flop & fsam & csam & fslo & cslo \\
\hline 18 & 44 & 0 & 0 & 0 & 0 & 2 & 4 & 0 & 0 \\
17 & 43 & 0 & 1 & 0 & 0 & 4 & 5 & 1 & 3 \\
16 & 76 & 0 & 1 & 0 & 0 & 8 & 22 & 10 & 11 \\
15 & 55 & 1 & 1 & 0 & 0 & 4 & 16 & 11 & 33 \\
14 & 216 & 0 & 0 & 0 & 0 & 42 & 19 & 21 & 52 \\
13 & 139 & 0 & 0 & 0 & 0 & 24 & 5 & 13 & 27 \\
12 & 64 & 2 & 6 & 1 & 2 & 34 & 25 & 17 & 35 \\
11 & 232 & 2 & 6 & 2 & 5 & 27 & 26 & 39 & 45 \\
8 & 86 & 1 & 2 & 2 & 0 & 25 & 32 & 9 & 8 \\
7 & 19 & 0 & 3 & 0 & 0 & 1 & 7 & 5 & 4 \\
6 & 56 & 0 & 5 & 1 & 0 & 3 & 13 & 4 & 9 \\
5 & 62 & 1 & 1 & 0 & 0 & 10 & 14 & 9 & 18 \\
4 & 101 & 1 & 4 & 2 & 1 & 38 & 58 & 12 & 27 \\
3 & 93 & 2 & 1 & 2 & 0 & 12 & 12 & 13 & 20 \\
2 & 47 & 0 & 2 & 0 & 0 & 9 & 6 & 9 & 21 \\
1 & 24 & 0 & 0 & 0 & 0 & 7 & 1 & 8 & 27 \\
\hline
\end{tabular}


mocness-1-cop222.raw WCR-82H MOCNESS tow copepods

$40^{-4}$

\begin{tabular}{rrrrrrrrrr} 
net\# & fbra & mbra & cbra & fdan & mdan & cdan & flam & clam & fvit \\
\hline 18 & 0 & 0 & 0 & 10 & 2 & 9 & 0 & 0 & 0 \\
17 & 0 & 0 & 2 & 7 & 5 & 13 & 0 & 0 & 0 \\
16 & 0 & 0 & 0 & 11 & 5 & 27 & 0 & 0 & 0 \\
15 & 1 & 0 & 0 & 3 & 3 & 27 & 5 & 7 & 0 \\
14 & 6 & 0 & 4 & 2 & 3 & 11 & 73 & 26 & 0 \\
13 & 3 & 3 & 3 & 0 & 0 & 6 & 53 & 17 & 0 \\
12 & 3 & 2 & 8 & 0 & 0 & 10 & 22 & 6 & 0 \\
11 & 18 & 5 & 24 & 5 & 0 & 8 & 9 & 7 & 0 \\
8 & 5 & 0 & 2 & 0 & 0 & 1 & 2 & 1 & 0 \\
7 & 3 & 1 & 0 & 0 & 0 & 0 & 0 & 0 & 0 \\
6 & 3 & 0 & 0 & 0 & 0 & 0 & 0 & 10 & 2 \\
5 & 1 & 0 & 0 & 0 & 0 & 0 & 1 & 17 & 7 \\
4 & 3 & 0 & 0 & 1 & 0 & 0 & 3 & 32 & 10 \\
3 & 3 & 0 & 1 & 0 & 0 & 0 & 8 & 17 & 7 \\
2 & 4 & 0 & 0 & 0 & 0 & 0 & 8 & 0 & 9 \\
1 & 0 & 0 & 1 & 0 & 0 & 0 & 3 & 37 & 6 \\
\hline
\end{tabular}

\begin{tabular}{crrrrrrrrr} 
net\# & fden & msca & csca & ftst & mtst & ctst & fven & mven & fbor \\
\hline 18 & 5 & 1 & 2 & 6 & 5 & 6 & 0 & 0 & 0 \\
17 & 9 & 2 & 6 & 0 & 1 & 2 & 0 & 0 & 0 \\
16 & 4 & 5 & 2 & 5 & 0 & 1 & 0 & 0 & 0 \\
15 & 4 & 3 & 3 & 1 & 0 & 2 & 0 & 0 & 0 \\
14 & 2 & 5 & 1 & 0 & 0 & 12 & 0 & 0 & 0 \\
13 & 1 & 11 & 2 & 0 & 0 & 2 & 0 & 0 & 0 \\
12 & 2 & 2 & 8 & 0 & 0 & 0 & 0 & 0 & 0 \\
11 & 3 & 8 & 10 & 0 & 0 & 0 & 0 & 0 & 1 \\
8 & 2 & 0 & 17 & 0 & 0 & 0 & 0 & 0 & 0 \\
7 & 1 & 2 & 1 & 0 & 0 & 0 & 0 & 0 & 0 \\
6 & 7 & 1 & 3 & 0 & 0 & 0 & 0 & 0 & 0 \\
5 & 4 & 5 & 1 & 0 & 0 & 0 & 0 & 0 & 0 \\
4 & 1 & 20 & 5 & 0 & 0 & 0 & 0 & 0 & 0 \\
3 & 5 & 24 & 4 & 0 & 0 & 0 & 0 & 0 & 0 \\
2 & 0 & 4 & 1 & 0 & 0 & 0 & 0 & 0 & 0 \\
1 & 0 & 5 & 10 & 0 & 0 & 0 & 0 & 2 & 0 \\
\hline
\end{tabular}


mocness-1-cop222.raw WCR-82H MOCNESS tow copepods

$40-5$

net\# fgra mpsm fpis mpis cpsm fabd mabd fxip mxip

\begin{tabular}{rrrrrrrrrr}
\hline 18 & 76 & 2 & 47 & 10 & 10 & 17 & 4 & 9 & 0 \\
17 & 59 & 5 & 16 & 7 & 23 & 4 & 3 & 2 & 0 \\
16 & 47 & 31 & 11 & 3 & 30 & 19 & 5 & 5 & 1 \\
15 & 24 & 30 & 6 & 6 & 66 & 18 & 2 & 5 & 0 \\
14 & 20 & 94 & 5 & 3 & 88 & 38 & 9 & 9 & 2 \\
13 & 14 & 27 & 2 & 2 & 13 & 18 & 5 & 5 & 3 \\
12 & 6 & 28 & 1 & 1 & 13 & 14 & 1 & 7 & 3 \\
11 & 3 & 32 & 2 & 1 & 2 & 10 & 5 & 24 & 9 \\
8 & 6 & 124 & 1 & 0 & 0 & 2 & 16 & 5 & 12 \\
7 & 1 & 4 & 0 & 0 & 1 & 0 & 1 & 0 & 0 \\
6 & 4 & 9 & 0 & 0 & 1 & 0 & 0 & 1 & 3 \\
5 & 8 & 14 & 1 & 1 & 0 & 6 & 2 & 4 & 1 \\
4 & 11 & 63 & 0 & 0 & 1 & 2 & 3 & 3 & 2 \\
3 & 6 & 13 & 0 & 0 & 5 & 3 & 3 & 2 & 0 \\
2 & 8 & 8 & 0 & 0 & 1 & 3 & 0 & 0 & 1 \\
1 & 7 & 3 & 0 & 0 & 3 & 3 & 2 & 0 & 0 \\
\hline
\end{tabular}

net\# cplg fvio mvio cvio flut mlut clut fpap mpap

\begin{tabular}{rrrrrrrrrr}
\hline 18 & 9 & 3 & 3 & 7 & 167 & 94 & 0 & 5 & 1 \\
17 & 17 & 4 & 2 & 4 & 123 & 66 & 0 & 14 & 8 \\
16 & 51 & 3 & 0 & 1 & 108 & 42 & 2 & 9 & 13 \\
15 & 76 & 0 & 0 & 1 & 78 & 47 & 2 & 9 & 8 \\
14 & 267 & 0 & 0 & 0 & 160 & 100 & 12 & 14 & 8 \\
13 & 96 & 0 & 0 & 0 & 65 & 24 & 10 & 4 & 8 \\
12 & 45 & 0 & 0 & 0 & 71 & 54 & 2 & 5 & 5 \\
11 & 30 & 0 & 0 & 0 & 97 & 49 & 5 & 6 & 1 \\
8 & 34 & 0 & 0 & 0 & 108 & 18 & 12 & 4 & 7 \\
7 & 16 & 0 & 0 & 0 & 11 & 5 & 3 & 7 & 11 \\
6 & 0 & 0 & 0 & 0 & 32 & 9 & 27 & 8 & 6 \\
5 & 12 & 0 & 0 & 0 & 36 & 12 & 32 & 12 & 32 \\
4 & 23 & 0 & 0 & 0 & 82 & 21 & 60 & 15 & 30 \\
3 & 11 & 0 & 0 & 0 & 68 & 9 & 11 & 16 & 18 \\
2 & 7 & 0 & 0 & 0 & 43 & 3 & 4 & 14 & 14 \\
1 & 9 & 0 & 0 & 0 & 45 & 22 & 8 & 10 & 11 \\
\hline
\end{tabular}


mocness-1-cop222.raw WCR-82H MOCNESS tow copepods

$40-6$

\begin{tabular}{rrrrrrrrrr} 
net\# & fspi & mspi & chet & fhal & mhal & chal & augs & fhel & mhel \\
\hline 18 & 0 & 0 & 0 & 1 & 0 & 0 & 0 & 0 & 0 \\
17 & 0 & 0 & 10 & 0 & 0 & 1 & 0 & 0 & 0 \\
16 & 1 & 1 & 19 & 0 & 0 & 0 & 1 & 0 & 0 \\
15 & 0 & 0 & 36 & 0 & 0 & 0 & 1 & 0 & 0 \\
14 & 0 & 0 & 83 & 50 & 0 & 1 & 9 & 0 & 0 \\
13 & 0 & 0 & 63 & 48 & 0 & 0 & 22 & 0 & 0 \\
12 & 0 & 0 & 28 & 218 & 1 & 1 & 14 & 0 & 0 \\
11 & 0 & 0 & 15 & 62 & 5 & 8 & 13 & 0 & 0 \\
8 & 4 & 1 & 20 & 25 & 1 & 88 & 10 & 4 & 0 \\
7 & 0 & 1 & 20 & 17 & 1 & 173 & 6 & 0 & 0 \\
6 & 1 & 2 & 46 & 9 & 5 & 200 & 11 & 1 & 0 \\
5 & 3 & 4 & 34 & 31 & 9 & 510 & 14 & 7 & 19 \\
4 & 8 & 7 & 43 & 23 & 4 & 736 & 51 & 17 & 30 \\
3 & 10 & 10 & 40 & 45 & 3 & 617 & 17 & 7 & 6 \\
2 & 5 & 4 & 35 & 30 & 0 & 168 & 38 & 8 & 0 \\
1 & 3 & 0 & 33 & 20 & 0 & 213 & 22 & 5 & 41 \\
\hline
\end{tabular}

\begin{tabular}{cccccccccc} 
net\# & fcur & mcur & meth & fket & mket & flon & mlon & fpak & mpak \\
\hline 18 & 0 & 0 & 0 & 0 & 0 & 0 & 0 & 1 & 2 \\
17 & 0 & 0 & 1 & 0 & 0 & 0 & 0 & 1 & 0 \\
16 & 0 & 0 & 0 & 0 & 0 & 4 & 0 & 0 & 1 \\
15 & 0 & 0 & 0 & 0 & 0 & 6 & 4 & 1 & 1 \\
14 & 0 & 0 & 0 & 0 & 0 & 4 & 4 & 1 & 1 \\
13 & 0 & 0 & 0 & 0 & 0 & 3 & 3 & 0 & 1 \\
12 & 0 & 0 & 0 & 0 & 0 & 0 & 1 & 1 & 1 \\
11 & 1 & 0 & 0 & 0 & 0 & 2 & 0 & 0 & 0 \\
8 & 0 & 0 & 0 & 0 & 0 & 2 & 1 & 0 & 0 \\
7 & 0 & 0 & 0 & 0 & 0 & 2 & 0 & 0 & 0 \\
6 & 1 & 0 & 0 & 4 & 0 & 0 & 0 & 0 & 0 \\
5 & 1 & 0 & 0 & 6 & 0 & 0 & 0 & 0 & 0 \\
4 & 0 & 0 & 0 & 4 & 1 & 0 & 0 & 0 & 0 \\
3 & 0 & 0 & 0 & 0 & 3 & 0 & 0 & 0 & 0 \\
2 & 0 & 0 & 0 & 0 & 1 & 0 & 0 & 0 & 0 \\
1 & 0 & 0 & 0 & 2 & 2 & 0 & 0 & 0 & 0 \\
\hline
\end{tabular}


mocness-1-cop222.raw WCR-82H MOCNESS tow copepods

$40-7$

\begin{tabular}{rrrrrrrrrr} 
net\# & fpbi & mpbi & ccan & fpon & mpon & cpon & fadn & mada & fneg \\
\hline 18 & 11 & 8 & 13 & 1 & 0 & 2 & 45 & 1 & 0 \\
17 & 7 & 17 & 19 & 0 & 0 & 3 & 17 & 1 & 0 \\
16 & 12 & 6 & 16 & 0 & 2 & 2 & 4 & 0 & 0 \\
15 & 14 & 7 & 21 & 0 & 1 & 1 & 4 & 0 & 0 \\
14 & 8 & 2 & 31 & 1 & 0 & 8 & 7 & 0 & 0 \\
13 & 5 & 6 & 0 & 1 & 1 & 6 & 16 & 0 & 0 \\
12 & 11 & 12 & 65 & 5 & 3 & 1 & 3 & 0 & 0 \\
11 & 11 & 9 & 42 & 5 & 2 & 0 & 7 & 0 & 0 \\
8 & 11 & 13 & 20 & 0 & 0 & 0 & 1 & 0 & 3 \\
7 & 6 & 2 & 1 & 0 & 0 & 0 & 0 & 0 & 0 \\
6 & 0 & 3 & 1 & 0 & 0 & 0 & 0 & 0 & 1 \\
5 & 0 & 0 & 1 & 0 & 0 & 0 & 0 & 0 & 1 \\
4 & 0 & 0 & 3 & 0 & 0 & 0 & 0 & 0 & 2 \\
3 & 0 & 0 & 2 & 0 & 0 & 0 & 0 & 0 & 1 \\
2 & 0 & 0 & 3 & 0 & 0 & 0 & 0 & 0 & 0 \\
1 & 0 & 0 & 1 & 0 & 0 & 0 & 0 & 0 & 2 \\
\hline
\end{tabular}

\begin{tabular}{rrrrrr} 
net\# & mneg & misc & oith & cycl & totl \\
\hline 18 & 0 & 18 & 8 & 142 & 1218 \\
17 & 0 & 4 & 29 & 218 & 1198 \\
16 & 0 & 15 & 34 & 259 & 1401 \\
15 & 0 & 13 & 14 & 280 & 1454 \\
14 & 0 & 175 & 304 & 455 & 3341 \\
13 & 0 & 101 & 158 & 170 & 1590 \\
12 & 0 & 47 & 100 & 193 & 1646 \\
11 & 0 & 70 & 133 & 148 & 1934 \\
8 & 2 & 71 & 232 & 132 & 1568 \\
7 & 0 & 40 & 43 & 151 & 636 \\
6 & 0 & 94 & 18 & 110 & 808 \\
5 & 0 & 82 & 17 & 84 & 1302 \\
4 & 0 & 104 & 16 & 146 & 2134 \\
3 & 0 & 87 & 40 & 135 & 1604 \\
2 & 0 & 47 & 12 & 81 & 799 \\
1 & 0 & 54 & 44 & 84 & 955 \\
\hline
\end{tabular}


mocness-1-cop222.std WCR-82H MOCNESS tow copepods

$4 p-1$

net\# midd depi volf aliq \#ften \#mten \#cten \#fnro \#mnro

\begin{tabular}{rrrrrrrrrr}
\hline 18 & 5 & 10 & 244 & 8 & 0 & 0 & 0 & 327 & 0 \\
17 & 15 & 10 & 177 & 8 & 0 & 0 & 0 & 45 & 0 \\
16 & 25 & 10 & 185 & 8 & 0 & 0 & 0 & 216 & 0 \\
15 & 35 & 10 & 227 & 8 & 35 & 0 & 70 & 141 & 0 \\
14 & 45 & 10 & 265 & 8 & 121 & 0 & 242 & 91 & 0 \\
13 & 55 & 10 & 220 & 8 & 73 & 0 & 109 & 0 & 0 \\
12 & 65 & 10 & 231 & 8 & 2811 & 35 & 1180 & 347 & 0 \\
11 & 75 & 10 & 339 & 8 & 2690 & 142 & 1935 & 71 & 0 \\
8 & 85 & 10 & 218 & 4 & 367 & 477 & 771 & 37 & 0 \\
7 & 95 & 10 & 225 & 4 & 53 & 107 & 53 & 18 & 0 \\
6 & 105 & 10 & 217 & 2 & 0 & 111 & 18 & 0 & 0 \\
5 & 115 & 10 & 167 & 1 & 0 & 126 & 6 & 12 & 0 \\
4 & 125 & 10 & 282 & 1 & 11 & 50 & 14 & 0 & 0 \\
3 & 135 & 10 & 284 & 1 & 0 & 18 & 0 & 11 & 0 \\
2 & 145 & 10 & 221 & 1 & 0 & 18 & 5 & 5 & 0 \\
1 & 155 & 10 & 181 & 1 & 6 & 17 & 17 & 0 & 6 \\
\hline
\end{tabular}

net\# \#cneo \#fund \#mund \#cund \#ccal \#fmin \#mmin \#cmin \#felg

\begin{tabular}{rrrrrrrrrr}
\hline 18 & 33 & 1277 & 949 & 1309 & 426 & 2717 & 1408 & 687 & 0 \\
17 & 634 & 362 & 498 & 2579 & 2127 & 2579 & 1629 & 1674 & 0 \\
16 & 690 & 129 & 302 & 4272 & 1553 & 2244 & 1381 & 4531 & 0 \\
15 & 1937 & 141 & 70 & 1866 & 1092 & 1585 & 1655 & 3838 & 0 \\
14 & 1268 & 151 & 211 & 543 & 2838 & 2385 & 1781 & 3200 & 0 \\
13 & 729 & 255 & 73 & 73 & 1130 & 1130 & 838 & 948 & 0 \\
12 & 798 & 312 & 208 & 0 & 1145 & 972 & 729 & 521 & 0 \\
11 & 590 & 142 & 24 & 0 & 944 & 566 & 212 & 354 & 0 \\
8 & 92 & 0 & 0 & 0 & 128 & 18 & 0 & 73 & 0 \\
7 & 53 & 0 & 0 & 0 & 89 & 18 & 0 & 0 & 0 \\
6 & 46 & 0 & 0 & 0 & 18 & 0 & 0 & 0 & 0 \\
5 & 66 & 0 & 0 & 0 & 78 & 0 & 0 & 0 & 0 \\
4 & 21 & 0 & 0 & 4 & 85 & 0 & 0 & 0 & 0 \\
3 & 35 & 0 & 0 & 4 & 113 & 0 & 0 & 0 & 0 \\
2 & 50 & 0 & 0 & 5 & 27 & 0 & 5 & 5 & 0 \\
1 & 28 & 6 & 0 & 11 & 143 & 11 & 0 & 17 & 0 \\
\hline
\end{tabular}


mocness-1-cop222.std WCR-82H MOCNESS tow copepods

$4 p-2$

net\# \#celg \#fatt \#matt \#catt \#fmey \#facr \#fpav \#farc \#marc

\begin{tabular}{rrrrrrrrrr}
\hline 18 & 0 & 33 & 0 & 229 & 0 & 524 & 360 & 851 & 0 \\
17 & 0 & 226 & 45 & 407 & 0 & 1674 & 453 & 769 & 181 \\
16 & 0 & 259 & 0 & 906 & 0 & 0 & 129 & 1165 & 43 \\
15 & 0 & 70 & 70 & 528 & 0 & 176 & 106 & 1585 & 141 \\
14 & 0 & 60 & 0 & 1177 & 0 & 393 & 30 & 3140 & 60 \\
13 & 0 & 109 & 182 & 510 & 182 & 0 & 0 & 1713 & 73 \\
12 & 0 & 139 & 174 & 902 & 208 & 0 & 104 & 2291 & 69 \\
11 & 0 & 71 & 24 & 401 & 118 & 0 & 71 & 3822 & 94 \\
8 & 0 & 18 & 0 & 18 & 0 & 0 & 495 & 936 & 0 \\
7 & 0 & 18 & 0 & 0 & 0 & 0 & 0 & 53 & 0 \\
6 & 0 & 0 & 0 & 0 & 0 & 0 & 9 & 83 & 0 \\
5 & 0 & 0 & 0 & 0 & 0 & 0 & 0 & 48 & 0 \\
4 & 0 & 7 & 0 & 0 & 0 & 0 & 4 & 68 & 0 \\
3 & 4 & 0 & 0 & 4 & 4 & 0 & 0 & 56 & 0 \\
2 & 0 & 0 & 0 & 0 & 0 & 0 & 5 & 45 & 0 \\
1 & 0 & 0 & 0 & 0 & 6 & 0 & 0 & 22 & 0 \\
\hline
\end{tabular}

net\# \#carc \#furc \#faar \#maar \#fege \#cege \#fgmi \#cgmi \#feme

\begin{tabular}{rrrrrrrrrr}
\hline 18 & 426 & 589 & 0 & 0 & 0 & 0 & 0 & 0 & 0 \\
17 & 996 & 453 & 0 & 0 & 0 & 0 & 0 & 0 & 45 \\
16 & 518 & 173 & 0 & 0 & 0 & 0 & 0 & 0 & 216 \\
15 & 423 & 35 & 0 & 0 & 0 & 0 & 0 & 0 & 141 \\
14 & 91 & 0 & 0 & 0 & 0 & 0 & 0 & 0 & 60 \\
13 & 146 & 36 & 0 & 0 & 0 & 0 & 0 & 0 & 0 \\
12 & 69 & 35 & 0 & 0 & 278 & 69 & 35 & 35 & 69 \\
11 & 94 & 0 & 0 & 24 & 873 & 1038 & 165 & 94 & 24 \\
8 & 220 & 92 & 18 & 110 & 184 & 2037 & 275 & 294 & 0 \\
7 & 0 & 36 & 18 & 107 & 71 & 89 & 36 & 160 & 0 \\
6 & 0 & 9 & 0 & 28 & 9 & 74 & 74 & 212 & 0 \\
5 & 24 & 12 & 0 & 6 & 18 & 12 & 54 & 347 & 0 \\
4 & 0 & 7 & 11 & 0 & 11 & 7 & 53 & 625 & 0 \\
3 & 0 & 0 & 28 & 0 & 18 & 4 & 14 & 342 & 0 \\
2 & 0 & 5 & 0 & 50 & 5 & 0 & 14 & 262 & 0 \\
1 & 0 & 110 & 0 & 39 & 6 & 0 & 11 & 491 & 6 \\
\hline
\end{tabular}


mocness-1-cop222.std WCR-82H MOCNESS tow copepods

$4 p \cdot 3$

net\# \#meme \#fros \#cuch \#fchi \#mchi \#fupl \#mupl \#feuk \#meuk

\begin{tabular}{|c|c|c|c|c|c|c|c|c|c|}
\hline 18 & 0 & 0 & 0 & 0 & 0 & 164 & 0 & 557 & 393 \\
\hline 17 & 0 & 0 & 45 & 0 & 0 & 317 & 0 & 679 & 91 \\
\hline 16 & 86 & 0 & 475 & 0 & 0 & 43 & 0 & 173 & 173 \\
\hline 15 & 0 & 0 & 1056 & 0 & 0 & 282 & 0 & 141 & 141 \\
\hline 14 & 60 & 0 & 8060 & 0 & 0 & 121 & 0 & 211 & 91 \\
\hline 13 & 0 & 0 & 5248 & 0 & 0 & 109 & 0 & 182 & 36 \\
\hline 12 & 35 & 0 & 937 & 35 & 0 & 69 & 0 & 382 & 139 \\
\hline 11 & 24 & 0 & 118 & 71 & 0 & 71 & 0 & 236 & 47 \\
\hline 8 & 0 & 0 & 92 & 55 & 0 & 37 & 0 & 110 & 73 \\
\hline 7 & 0 & 18 & 18 & 18 & 18 & 53 & 0 & 71 & 0 \\
\hline 6 & 0 & 0 & 9 & 18 & 46 & 18 & 0 & 9 & 0 \\
\hline 5 & 0 & 0 & 60 & 0 & 30 & 0 & 0 & 30 & 0 \\
\hline 4 & 0 & 0 & 64 & 7 & 11 & 11 & 0 & 7 & 4 \\
\hline 3 & 0 & 0 & 0 & 7 & 7 & 7 & 0 & 4 & 0 \\
\hline 2 & 0 & 0 & 118 & 5 & 0 & 0 & 0 & 14 & 0 \\
\hline 1 & 0 & 0 & -999 & 0 & 0 & 6 & 0 & 6 & 6 \\
\hline
\end{tabular}

net\# \#ceuk \#fpsp \#cpsp \#fsco \#flop \#fsam \#csam \#fslo \#cslo

\begin{tabular}{|c|c|c|c|c|c|c|c|c|c|}
\hline 18 & 1440 & 0 & 0 & 0 & 0 & 66 & 131 & 0 & 0 \\
\hline 17 & 1946 & 0 & 45 & 0 & 0 & 181 & 226 & 45 & 136 \\
\hline 16 & 3279 & 0 & 43 & 0 & 0 & 345 & 949 & 432 & 475 \\
\hline 15 & 1937 & 35 & 35 & 0 & 0 & 141 & 563 & 387 & 1162 \\
\hline 14 & 6521 & 0 & 0 & 0 & 0 & 1268 & 574 & 634 & 1570 \\
\hline 13 & 5066 & 0 & 0 & 0 & 0 & 875 & 182 & 474 & 984 \\
\hline 12 & 2221 & 69 & 208 & 35 & 69 & 1180 & 868 & 590 & 1215 \\
\hline 11 & 5473 & 47 & 142 & 47 & 118 & 637 & 613 & 920 & 1062 \\
\hline 8 & 1578 & 18 & 37 & 37 & 0 & 459 & 587 & 165 & 147 \\
\hline 7 & 338 & 0 & 53 & 0 & 0 & 18 & 125 & 89 & 71 \\
\hline 6 & 516 & 0 & 46 & 9 & 0 & 28 & 120 & 37 & 83 \\
\hline 5 & 371 & 6 & 6 & 0 & 0 & 60 & 84 & 54 & 108 \\
\hline 4 & 359 & 4 & 14 & 7 & 4 & 135 & 206 & 43 & 96 \\
\hline 3 & 328 & 7 & 4 & 7 & 0 & 42 & 42 & 46 & 71 \\
\hline 2 & 213 & 0 & 9 & 0 & 0 & 41 & 27 & 41 & 95 \\
\hline 1 & 132 & 0 & 0 & 0 & 0 & 39 & 6 & 44 & 149 \\
\hline
\end{tabular}


mocness-1-cop222.std WCR-82H MOCNESS tow copepods

$4 p \cdot 4$

net\# \#fbra \#mbra \#cbra \#fdan \#mdan \#cdan \#flam \#clam \#fvit

\begin{tabular}{rrrrrrrrrr}
\hline 18 & 0 & 0 & 0 & 327 & 66 & 295 & 0 & 0 & 0 \\
17 & 0 & 0 & 91 & 317 & 226 & 588 & 0 & 0 & 0 \\
16 & 0 & 0 & 0 & 475 & 216 & 1165 & 0 & 0 & 0 \\
15 & 35 & 0 & 0 & 106 & 106 & 951 & 176 & 247 & 0 \\
14 & 181 & 0 & 121 & 60 & 91 & 332 & 2204 & 785 & 0 \\
13 & 109 & 109 & 109 & 0 & 0 & 219 & 1932 & 620 & 0 \\
12 & 104 & 69 & 278 & 0 & 0 & 347 & 764 & 208 & 0 \\
11 & 425 & 118 & 566 & 118 & 0 & 189 & 212 & 165 & 0 \\
8 & 92 & 0 & 37 & 0 & 0 & 18 & 37 & 18 & 0 \\
7 & 53 & 18 & 0 & 0 & 0 & 0 & 0 & 0 & 0 \\
6 & 28 & 0 & 0 & 0 & 0 & 0 & 0 & 92 & 18 \\
5 & 6 & 0 & 0 & 0 & 0 & 0 & 6 & 102 & 42 \\
4 & 11 & 0 & 0 & 4 & 0 & 0 & 11 & 114 & 36 \\
3 & 11 & 0 & 4 & 0 & 0 & 0 & 28 & 60 & 25 \\
2 & 18 & 0 & 0 & 0 & 0 & 0 & 36 & 0 & 41 \\
1 & 0 & 0 & 6 & 0 & 0 & 0 & 17 & 204 & 33 \\
\hline
\end{tabular}

net\# \#fden \#msca \#csca \#ftst \#mtst \#ctst \#fven \#mven \#fbor

\begin{tabular}{rrrrrrrrrr}
\hline 18 & 164 & 33 & 66 & 196 & 164 & 196 & 0 & 0 & 0 \\
17 & 407 & 91 & 272 & 0 & 45 & 91 & 0 & 0 & 0 \\
16 & 173 & 216 & 86 & 216 & 0 & 43 & 0 & 0 & 0 \\
15 & 141 & 106 & 106 & 35 & 0 & 70 & 0 & 0 & 0 \\
14 & 60 & 151 & 30 & 0 & 0 & 362 & 0 & 0 & 0 \\
13 & 36 & 401 & 73 & 0 & 0 & 73 & 0 & 0 & 0 \\
12 & 69 & 69 & 278 & 0 & 0 & 0 & 0 & 0 & 0 \\
11 & 71 & 189 & 236 & 0 & 0 & 0 & 0 & 0 & 24 \\
8 & 37 & 0 & 312 & 0 & 0 & 0 & 0 & 0 & 0 \\
7 & 18 & 36 & 18 & 0 & 0 & 0 & 0 & 0 & 0 \\
6 & 65 & 9 & 28 & 0 & 0 & 0 & 0 & 0 & 0 \\
5 & 24 & 30 & 6 & 0 & 0 & 0 & 0 & 0 & 0 \\
4 & 4 & 71 & 18 & 0 & 0 & 0 & 0 & 0 & 0 \\
3 & 18 & 85 & 14 & 0 & 0 & 0 & 0 & 0 & 0 \\
2 & 0 & 18 & 5 & 0 & 0 & 0 & 0 & 0 & 0 \\
1 & 0 & 28 & 55 & 0 & 0 & 0 & 0 & 11 & 0 \\
\hline
\end{tabular}


mocness-1-cop222.std WCR-82H MOCNESS tow copepods

$4 p-5$

net\# \#fgra \#mpsm \#fpis \#mpis \#cpsm \#fabd \#mabd \#fxip \#mxip

\begin{tabular}{rrrrrrrrrr}
\hline 18 & 2488 & 66 & 1539 & 327 & 327 & 557 & 131 & 295 & 0 \\
17 & 2670 & 226 & 724 & 317 & 1041 & 181 & 136 & 91 & 0 \\
16 & 2028 & 1338 & 475 & 129 & 1295 & 820 & 216 & 216 & 43 \\
15 & 845 & 1056 & 211 & 211 & 2324 & 634 & 70 & 176 & 0 \\
14 & 604 & 2838 & 151 & 91 & 2657 & 1147 & 272 & 272 & 60 \\
13 & 510 & 984 & 73 & 73 & 474 & 656 & 182 & 182 & 109 \\
12 & 208 & 972 & 35 & 35 & 451 & 486 & 35 & 243 & 104 \\
11 & 71 & 755 & 47 & 24 & 47 & 236 & 118 & 566 & 212 \\
8 & 110 & 2275 & 18 & 0 & 0 & 37 & 294 & 92 & 220 \\
7 & 18 & 71 & 0 & 0 & 18 & 0 & 18 & 0 & 0 \\
6 & 37 & 83 & 0 & 0 & 9 & 0 & 0 & 9 & 28 \\
5 & 48 & 84 & 6 & 6 & 0 & 36 & 12 & 24 & 6 \\
4 & 39 & 224 & 0 & 0 & 4 & 7 & 11 & 11 & 7 \\
3 & 21 & 46 & 0 & 0 & 18 & 11 & 11 & 7 & 0 \\
2 & 36 & 36 & 0 & 0 & 5 & 14 & 0 & 0 & 5 \\
1 & 39 & 17 & 0 & 0 & 17 & 17 & 11 & 0 & 0 \\
\hline
\end{tabular}

net\# \#cplg \#fvio \#mvio \#cvio \#flut \#mlut \#clut \#fpap \#mpap

\begin{tabular}{rrrrrrrrrr}
\hline 18 & 295 & 98 & 98 & 229 & 5466 & 3077 & 0 & 164 & 33 \\
17 & 769 & 181 & 91 & 181 & 5566 & 2986 & 0 & 634 & 362 \\
16 & 2201 & 129 & 0 & 43 & 4660 & 1812 & 86 & 388 & 561 \\
15 & 2676 & 0 & 0 & 35 & 2747 & 1655 & 70 & 317 & 282 \\
14 & 8060 & 0 & 0 & 0 & 4830 & 3019 & 362 & 423 & 242 \\
13 & 3499 & 0 & 0 & 0 & 2369 & 875 & 365 & 146 & 292 \\
12 & 1562 & 0 & 0 & 0 & 2464 & 1874 & 69 & 174 & 174 \\
11 & 708 & 0 & 0 & 0 & 2288 & 1156 & 118 & 142 & 24 \\
8 & 624 & 0 & 0 & 0 & 1982 & 330 & 220 & 73 & 128 \\
7 & 285 & 0 & 0 & 0 & 196 & 89 & 53 & 125 & 196 \\
6 & 0 & 0 & 0 & 0 & 295 & 83 & 249 & 74 & 55 \\
5 & 72 & 0 & 0 & 0 & 216 & 72 & 192 & 72 & 192 \\
4 & 82 & 0 & 0 & 0 & 291 & 75 & 213 & 53 & 107 \\
3 & 39 & 0 & 0 & 0 & 240 & 32 & 39 & 56 & 63 \\
2 & 32 & 0 & 0 & 0 & 194 & 14 & 18 & 63 & 63 \\
1 & 50 & 0 & 0 & 0 & 248 & 121 & 44 & 55 & 61 \\
\hline
\end{tabular}


mocness-1-cop222.std WCR-82H MOCNESS tow copepods

$4 p-6$

net\# \#fspi \#mspi \#chet \#fhal \#mhal \#chal \#augs \#fhel \#mhel

\begin{tabular}{rrrrrrrrrr}
\hline 18 & 0 & 0 & 0 & 33 & 0 & 0 & 0 & 0 & 0 \\
17 & 0 & 0 & 453 & 0 & 0 & 45 & 0 & 0 & 0 \\
16 & 43 & 43 & 820 & 0 & 0 & 0 & 43 & 0 & 0 \\
15 & 0 & 0 & 1268 & 0 & 0 & 0 & 35 & 0 & 0 \\
14 & 0 & 0 & 2506 & 1509 & 0 & 30 & 272 & 0 & 0 \\
13 & 0 & 0 & 2296 & 1749 & 0 & 0 & 802 & 0 & 0 \\
12 & 0 & 0 & 972 & 7566 & 35 & 35 & 486 & 0 & 0 \\
11 & 0 & 0 & 354 & 1463 & 118 & 189 & 307 & 0 & 0 \\
8 & 73 & 18 & 367 & 459 & 18 & 1615 & 184 & 73 & 0 \\
7 & 0 & 18 & 356 & 303 & 18 & 3081 & 107 & 0 & 0 \\
6 & 9 & 18 & 424 & 83 & 46 & 1842 & 101 & 9 & 0 \\
5 & 18 & 24 & 204 & 186 & 54 & 3054 & 84 & 42 & 114 \\
4 & 28 & 25 & 153 & 82 & 14 & 2615 & 181 & 60 & 107 \\
3 & 35 & 35 & 141 & 159 & 11 & 2175 & 60 & 25 & 21 \\
2 & 23 & 18 & 158 & 136 & 0 & 760 & 172 & 36 & 0 \\
1 & 17 & 0 & 182 & 110 & 0 & 1174 & 121 & 28 & 226 \\
\hline
\end{tabular}

net\# \#fcur \#mcur \#meth \#fket \#mket \#flon \#mlon \#fpak \#mpak

\begin{tabular}{rrrrrrrrrr}
\hline 18 & 0 & 0 & 0 & 0 & 0 & 0 & 0 & 33 & 66 \\
17 & 0 & 0 & 45 & 0 & 0 & 0 & 0 & 45 & 0 \\
16 & 0 & 0 & 0 & 0 & 0 & 173 & 0 & 0 & 43 \\
15 & 0 & 0 & 0 & 0 & 0 & 211 & 141 & 35 & 35 \\
14 & 0 & 0 & 0 & 0 & 0 & 121 & 121 & 30 & 30 \\
13 & 0 & 0 & 0 & 0 & 0 & 109 & 109 & 0 & 36 \\
12 & 0 & 0 & 0 & 0 & 0 & 0 & 35 & 35 & 35 \\
11 & 24 & 0 & 0 & 0 & 0 & 47 & 0 & 0 & 0 \\
8 & 0 & 0 & 0 & 0 & 0 & 37 & 18 & 0 & 0 \\
7 & 0 & 0 & 0 & 0 & 0 & 36 & 0 & 0 & 0 \\
6 & 9 & 0 & 0 & 37 & 0 & 0 & 0 & 0 & 0 \\
5 & 6 & 0 & 0 & 36 & 0 & 0 & 0 & 0 & 0 \\
4 & 0 & 0 & 0 & 14 & 4 & 0 & 0 & 0 & 0 \\
3 & 0 & 0 & 0 & 0 & 11 & 0 & 0 & 0 & 0 \\
2 & 0 & 0 & 0 & 0 & 5 & 0 & 0 & 0 & 0 \\
1 & 0 & 0 & 0 & 11 & 11 & 0 & 0 & 0 & 0 \\
\hline
\end{tabular}


mocness-1-cop222.std WCR-82H MOCNESS tow copepods

$4 p-7$

net\# \#fpbi \#mpbi \#ccan \#fpon \#mpon \#cpon \#fadn \#mada \#fneg

\begin{tabular}{rrrrrrrrrr}
\hline 18 & 360 & 262 & 426 & 33 & 0 & 66 & 1473 & 33 & 0 \\
17 & 317 & 769 & 860 & 0 & 0 & 136 & 769 & 45 & 0 \\
16 & 518 & 259 & 690 & 0 & 86 & 86 & 173 & 0 & 0 \\
15 & 493 & 247 & 739 & 0 & 35 & 35 & 141 & 0 & 0 \\
14 & 242 & 60 & 936 & 30 & 0 & 242 & 211 & 0 & 0 \\
13 & 182 & 219 & 0 & 36 & 36 & 219 & 583 & 0 & 0 \\
12 & 382 & 417 & 2256 & 174 & 104 & 35 & 104 & 0 & 0 \\
11 & 260 & 212 & 991 & 118 & 47 & 0 & 165 & 0 & 0 \\
8 & 202 & 239 & 367 & 0 & 0 & 0 & 18 & 0 & 55 \\
7 & 107 & 36 & 18 & 0 & 0 & 0 & 0 & 0 & 0 \\
6 & 0 & 28 & 9 & 0 & 0 & 0 & 0 & 0 & 9 \\
5 & 0 & 0 & 6 & 0 & 0 & 0 & 0 & 0 & 6 \\
4 & 0 & 0 & 11 & 0 & 0 & 0 & 0 & 0 & 7 \\
3 & 0 & 0 & 7 & 0 & 0 & 0 & 0 & 0 & 4 \\
2 & 0 & 0 & 14 & 0 & 0 & 0 & 0 & 0 & 0 \\
1 & 0 & 0 & 6 & 0 & 0 & 0 & 0 & 0 & 11 \\
\hline
\end{tabular}

net\# \#mneg \#misc \#oith \#cycl \#totl

\begin{tabular}{rrrrrr}
\hline 18 & 0 & 589 & 262 & 4648 & 39869 \\
17 & 0 & 181 & 1312 & 9864 & 54208 \\
16 & 0 & 647 & 1467 & 11176 & 60453 \\
15 & 0 & 458 & 493 & 9859 & 51197 \\
14 & 0 & 5283 & 9177 & 13736 & 100860 \\
13 & 0 & 3681 & 5759 & 6196 & 57950 \\
12 & 0 & 1631 & 3471 & 6699 & 57128 \\
11 & 0 & 1651 & 3138 & 3492 & 45627 \\
8 & 37 & 1303 & 4257 & 2422 & 28771 \\
7 & 0 & 712 & 766 & 2689 & 11327 \\
6 & 0 & 866 & 166 & 1013 & 7440 \\
5 & 0 & 491 & 102 & 503 & 7796 \\
4 & 0 & 369 & 57 & 519 & 7581 \\
3 & 0 & 307 & 141 & 476 & 5654 \\
2 & 0 & 213 & 54 & 366 & 3612 \\
1 & 0 & 298 & 243 & 463 & 5265 \\
\hline
\end{tabular}


mocness-1-cop222. int WCR-82H MOCNESS tow copepods

$4 q-1$

net\# midd depi volf aliq iften imten icten ifnro imnro

\begin{tabular}{rrrrrrrrrr}
\hline 18 & 5 & 10 & 244 & 8 & 0 & 0 & 0 & 3 & 0 \\
17 & 15 & 10 & 177 & 8 & 0 & 0 & 0 & 4 & 0 \\
16 & 25 & 10 & 185 & 8 & 0 & 0 & 0 & 6 & 0 \\
15 & 35 & 10 & 227 & 8 & 0 & 0 & 1 & 7 & 0 \\
14 & 45 & 10 & 265 & 8 & 2 & 0 & 3 & 8 & 0 \\
13 & 55 & 10 & 220 & 8 & 2 & 0 & 4 & 8 & 0 \\
12 & 65 & 10 & 231 & 8 & 30 & 0 & 16 & 12 & 0 \\
11 & 75 & 10 & 339 & 8 & 57 & 2 & 35 & 12 & 0 \\
8 & 85 & 10 & 218 & 4 & 61 & 7 & 43 & 13 & 0 \\
7 & 95 & 10 & 225 & 4 & 62 & 8 & 44 & 13 & 0 \\
6 & 105 & 10 & 217 & 2 & 62 & 9 & 44 & 13 & 0 \\
5 & 115 & 10 & 167 & 1 & 62 & 10 & 44 & 13 & 0 \\
4 & 125 & 10 & 282 & 1 & 62 & 10 & 44 & 13 & 0 \\
3 & 135 & 10 & 284 & 1 & 62 & 11 & 44 & 13 & 0 \\
2 & 145 & 10 & 221 & 1 & 62 & 11 & 44 & 13 & 0 \\
1 & 155 & 10 & 181 & 1 & 62 & 11 & 44 & 13 & 0 \\
\hline
\end{tabular}

net\# icneo ifund imund icund iccal ifmin immin icmin ifelg

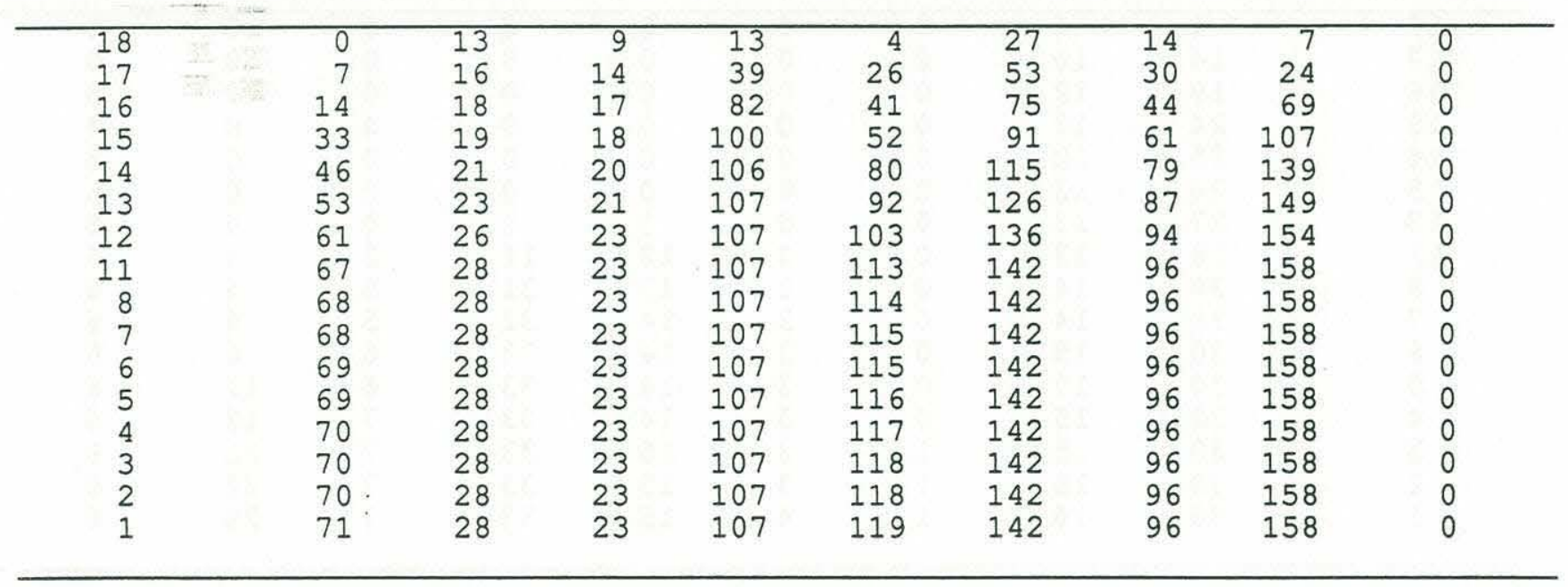


mocness-1-cop222. int WCR-82H MOCNESS tow copepods

$4 q^{2}$

net\# icelg ifatt imatt icatt ifmey ifacr ifpav ifarc imarc

\begin{tabular}{rrrrrrrrrr}
\hline 18 & 0 & 0 & 0 & 2 & 0 & 5 & 4 & 8 & 0 \\
17 & 0 & 3 & 0 & 6 & 0 & 22 & 8 & 16 & 2 \\
16 & 0 & 5 & 0 & 15 & 0 & 22 & 9 & 28 & 2 \\
15 & 0 & 6 & 1 & 21 & 0 & 24 & 10 & 44 & 4 \\
14 & 0 & 6 & 1 & 32 & 0 & 28 & 11 & 75 & 4 \\
13 & 0 & 8 & 3 & 38 & 2 & 28 & 11 & 92 & 5 \\
12 & 0 & 9 & 5 & 47 & 4 & 28 & 12 & 115 & 6 \\
11 & 0 & 10 & 5 & 51 & 5 & 28 & 13 & 153 & 7 \\
8 & 0 & 10 & 5 & 51 & 5 & 28 & 17 & 163 & 7 \\
7 & 0 & 10 & 5 & 51 & 5 & 28 & 17 & 163 & 7 \\
6 & 0 & 10 & 5 & 51 & 5 & 28 & 18 & 164 & 7 \\
5 & 0 & 10 & 5 & 51 & 5 & 28 & 18 & 165 & 7 \\
4 & 0 & 10 & 5 & 51 & 5 & 28 & 18 & 165 & 7 \\
3 & 0 & 10 & 5 & 51 & 5 & 28 & 18 & 166 & 7 \\
2 & 0 & 10 & 5 & 51 & 5 & 28 & 18 & 166 & 7 \\
1 & 0 & 10 & 5 & 51 & 5 & 28 & 18 & 166 & 7 \\
\hline
\end{tabular}

net\# icarc ifurc ifaar imaar ifege icege ifgmi icgmi ifeme

\begin{tabular}{rrrrrrrrrr}
\hline 18 & 4 & 6 & 0 & 0 & 0 & 0 & 0 & 0 & 0 \\
17 & 14 & 10 & 0 & 0 & 0 & 0 & 0 & 0 & 0 \\
16 & 19 & 12 & 0 & 0 & 0 & 0 & 0 & 0 & 3 \\
15 & 24 & 12 & 0 & 0 & 0 & 0 & 0 & 0 & 4 \\
14 & 25 & 12 & 0 & 0 & 0 & 0 & 0 & 0 & 5 \\
13 & 26 & 13 & 0 & 0 & 0 & 0 & 0 & 0 & 5 \\
12 & 27 & 13 & 0 & 0 & 3 & 1 & 0 & 0 & 5 \\
11 & 28 & 13 & 0 & 0 & 12 & 11 & 2 & 1 & 6 \\
8 & 30 & 14 & 0 & 1 & 13 & 31 & 5 & 4 & 6 \\
7 & 30 & 14 & 0 & 2 & 14 & 32 & 5 & 6 & 6 \\
6 & 30 & 15 & 0 & 3 & 14 & 33 & 6 & 8 & 6 \\
5 & 30 & 15 & 0 & 3 & 14 & 33 & 6 & 11 & 6 \\
4 & 30 & 15 & 0 & 3 & 14 & 33 & 7 & 18 & 6 \\
3 & 30 & 15 & 1 & 3 & 15 & 33 & 7 & 21 & 6 \\
2 & 30 & 15 & 1 & 3 & 15 & 33 & 7 & 24 & 6 \\
1 & 30 & 16 & 1 & 4 & 15 & 33 & 7 & 29 & 6 \\
\hline
\end{tabular}


mocness-1-cop222. int WCR-82H MOCNESS tow copepods

$4 q-3$

net\# imeme ifros icuch ifchi imchi ifupl imupl ifeuk imeuk

\begin{tabular}{rrrrrrrrrr}
\hline 18 & 0 & 0 & 0 & 0 & 0 & 2 & 0 & 6 & 4 \\
17 & 0 & 0 & 0 & 0 & 0 & 5 & 0 & 12 & 5 \\
16 & 1 & 0 & 5 & 0 & 0 & 5 & 0 & 14 & 7 \\
15 & 1 & 0 & 16 & 0 & 0 & 8 & 0 & 15 & 8 \\
14 & 1 & 0 & 96 & 0 & 0 & 9 & 0 & 18 & 9 \\
13 & 1 & 0 & 149 & 0 & 0 & 10 & 0 & 19 & 9 \\
12 & 2 & 0 & 158 & 0 & 0 & 11 & 0 & 23 & 11 \\
11 & 2 & 0 & 159 & 1 & 0 & 12 & 0 & 26 & 11 \\
8 & 2 & 0 & 160 & 2 & 0 & 12 & 0 & 27 & 12 \\
7 & 2 & 0 & 161 & 2 & 0 & 13 & 0 & 27 & 12 \\
6 & 2 & 0 & 161 & 2 & 1 & 13 & 0 & 27 & 12 \\
5 & 2 & 0 & 161 & 2 & 1 & 13 & 0 & 28 & 12 \\
4 & 2 & 0 & 162 & 2 & 1 & 13 & 0 & 28 & 12 \\
3 & 2 & 0 & 162 & 2 & 1 & 13 & 0 & 28 & 12 \\
2 & 2 & 0 & 163 & 2 & 1 & 13 & 0 & 28 & 12 \\
1 & 2 & 0 & 153 & 2 & 1 & 13 & 0 & 28 & 12 \\
\hline
\end{tabular}

\begin{tabular}{rrrrrrrrrr} 
net\# & iceuk & ifpsp & icpsp & ifsco & iflop & ifsam & icsam & ifslo & icslo \\
\hline 18 & 14 & 0 & 0 & 0 & 0 & 1 & 1 & 0 & 0 \\
17 & 34 & 0 & 0 & 0 & 0 & 2 & 4 & 0 & 1 \\
16 & 66 & 0 & 1 & 0 & 0 & 6 & 13 & 5 & 6 \\
15 & 86 & 0 & 1 & 0 & 0 & 7 & 19 & 9 & 18 \\
14 & 151 & 0 & 1 & 0 & 0 & 20 & 24 & 15 & 33 \\
13 & 202 & 0 & 1 & 0 & 0 & 29 & 26 & 20 & 43 \\
12 & 224 & 1 & 3 & 0 & 1 & 41 & 35 & 26 & 56 \\
11 & 279 & 2 & 5 & 1 & 2 & 47 & 41 & 35 & 66 \\
8 & 295 & 2 & 5 & 1 & 2 & 52 & 47 & 36 & 68 \\
7 & 298 & 2 & 6 & 1 & 2 & 52 & 48 & 37 & 68 \\
6 & 303 & 2 & 6 & 1 & 2 & 52 & 49 & 38 & 69 \\
5 & 307 & 2 & 6 & 1 & 2 & 53 & 50 & 38 & 70 \\
4 & 311 & 2 & 6 & 1 & 2 & 54 & 52 & 39 & 71 \\
3 & 314 & 2 & 6 & 1 & 2 & 54 & 53 & 39 & 72 \\
2 & 316 & 2 & 6 & 1 & 2 & 55 & 53 & 40 & 73 \\
1 & 317 & 2 & 6 & 1 & 2 & 55 & 53 & 40 & 74 \\
\hline
\end{tabular}


mocness-1-cop222. int WCR-82H MOCNESS tow copepods

$4 q-4$

\begin{tabular}{rrrrrrrrrr} 
net\# & ifbra & imbra & icbra & ifdan & imdan & icdan & iflam & iclam & ifvit \\
\hline 18 & 0 & 0 & 0 & 3 & 1 & 3 & 0 & 0 & 0 \\
17 & 0 & 0 & 1 & 6 & 3 & 9 & 0 & 0 & 0 \\
16 & 0 & 0 & 1 & 11 & 5 & 20 & 0 & 0 & 0 \\
15 & 0 & 0 & 1 & 12 & 6 & 30 & 2 & 2 & 0 \\
14 & 2 & 0 & 2 & 13 & 7 & 33 & 24 & 10 & 0 \\
13 & 3 & 1 & 3 & 13 & 7 & 35 & 43 & 16 & 0 \\
12 & 4 & 2 & 6 & 13 & 7 & 39 & 51 & 19 & 0 \\
11 & 9 & 3 & 12 & 14 & 7 & 41 & 53 & 20 & 0 \\
8 & 9 & 3 & 12 & 14 & 7 & 41 & 53 & 20 & 0 \\
7 & 10 & 3 & 12 & 14 & 7 & 41 & 53 & 20 & 0 \\
6 & 10 & 3 & 12 & 14 & 7 & 41 & 53 & 21 & 0 \\
5 & 10 & 3 & 12 & 14 & 7 & 41 & 53 & 22 & 1 \\
4 & 10 & 3 & 12 & 14 & 7 & 41 & 53 & 23 & 1 \\
3 & 11 & 3 & 12 & 14 & 7 & 41 & 54 & 24 & 1 \\
2 & 11 & 3 & 12 & 14 & 7 & 41 & 54 & 24 & 2 \\
1 & 11 & 3 & 12 & 14 & 7 & 41 & 54 & 26 & 2 \\
\hline
\end{tabular}

\begin{tabular}{|c|c|c|c|c|c|c|c|c|c|}
\hline net\# & ifden & imsca & icsca & iftst & imtst & ictst & ifven & imven & ifbor \\
\hline 18 & 2 & 0 & 1 & 2 & 2 & 2 & 0 & 0 & 0 \\
\hline 17 & 6 & 1 & 3 & 2 & 2 & 3 & 0 & 0 & 0 \\
\hline 16 & 7 & 3 & 4 & 4 & 2 & 3 & 0 & 0 & 0 \\
\hline 15 & 9 & 4 & 5 & 4 & 2 & 4 & 0 & 0 & 0 \\
\hline 14 & 9 & 6 & 6 & 4 & 2 & 8 & 0 & 0 & 0 \\
\hline 13 & 10 & 10 & 6 & 4 & 2 & 8 & 0 & 0 & 0 \\
\hline 12 & 11 & 11 & 9 & 4 & 2 & 8 & 0 & 0 & 0 \\
\hline 11 & 11 & 13 & 11 & 4 & 2 & 8 & 0 & 0 & 0 \\
\hline 8 & 12 & 13 & 15 & 4 & 2 & 8 & 0 & 0 & 0 \\
\hline 7 & 12 & 13 & 15 & 4 & 2 & 8 & 0 & 0 & 0 \\
\hline 6 & 12 & 13 & 15 & 4 & 2 & 8 & 0 & 0 & 0 \\
\hline 5 & 13 & 13 & 15 & 4 & 2 & 8 & 0 & 0 & 0 \\
\hline 4 & 13 & 14 & 15 & 4 & 2 & 8 & 0 & 0 & 0 \\
\hline 3 & 13 & 15 & 15 & 4 & 2 & 8 & 0 & 0 & 0 \\
\hline 2 & 13 & 15 & 15 & 4 & 2 & 8 & 0 & 0 & 0 \\
\hline 1 & 13 & 15 & 16 & 4 & 2 & 8 & 0 & 0 & 0 \\
\hline
\end{tabular}


mocness-1-cop222. int WCR-82H MOCNESS tow copepods

$4 q \cdot 5$

net\# ifgra impsm ifpis impis icpsm ifabd imabd ifxip imxip

\begin{tabular}{rrrrrrrrrr}
\hline 18 & 25 & 1 & 15 & 3 & 3 & 6 & 1 & 3 & 0 \\
17 & 52 & 3 & 23 & 6 & 14 & 7 & 3 & 4 & 0 \\
16 & 72 & 16 & 27 & 8 & 27 & 16 & 5 & 6 & 0 \\
15 & 80 & 27 & 29 & 10 & 50 & 22 & 6 & 8 & 0 \\
14 & 86 & 55 & 31 & 11 & 77 & 33 & 8 & 10 & 1 \\
13 & 91 & 65 & 32 & 11 & 81 & 40 & 10 & 12 & 2 \\
12 & 94 & 75 & 32 & 12 & 86 & 45 & 10 & 15 & 3 \\
11 & 94 & 82 & 33 & 12 & 86 & 47 & 12 & 20 & 5 \\
8 & 95 & 105 & 33 & 12 & 86 & 48 & 15 & 21 & 7 \\
7 & 95 & 106 & 33 & 12 & 86 & 48 & 15 & 21 & 7 \\
6 & 96 & 106 & 33 & 12 & 86 & 48 & 15 & 21 & 8 \\
5 & 96 & 107 & 33 & 12 & 86 & 48 & 15 & 22 & 8 \\
4 & 97 & 110 & 33 & 12 & 86 & 48 & 15 & 22 & 8 \\
3 & 97 & 110 & 33 & 12 & 87 & 48 & 15 & 22 & 8 \\
2 & 97 & 110 & 33 & 12 & 87 & 48 & 15 & 22 & 8 \\
1 & 98 & 110 & 33 & 12 & 87 & 48 & 15 & 22 & 8 \\
\hline
\end{tabular}

\begin{tabular}{|c|c|c|c|c|c|c|c|c|c|}
\hline net\# & icplg & ifvio & imvio & icvio & iflut & imlut & iclut & ifpap & impap \\
\hline 18 & 3 & 1 & 1 & 2 & 55 & 31 & 0 & 2 & 0 \\
\hline 17 & 11 & 3 & 2 & 4 & 110 & 61 & 0 & 8 & 4 \\
\hline 16 & 33 & 4 & 2 & 5 & 157 & 79 & 1 & 12 & 10 \\
\hline 15 & 60 & 4 & 2 & 5 & 185 & 95 & 2 & 15 & 12 \\
\hline 14 & 140 & 4 & 2 & 5 & 233 & 126 & 5 & 19 & 15 \\
\hline 13 & 175 & 4 & 2 & 5 & 257 & 134 & 9 & 21 & 18 \\
\hline 12 & 191 & 4 & 2 & 5 & 281 & 153 & 10 & 22 & 19 \\
\hline 11 & 198 & 4 & 2 & 5 & 304 & 165 & 11 & 24 & 20 \\
\hline 8 & 204 & 4 & 2 & 5 & 324 & 168 & 13 & 25 & 21 \\
\hline 7 & 207 & 4 & 2 & 5 & 326 & 169 & 13 & 26 & 23 \\
\hline 6 & 207 & 4 & 2 & 5 & 329 & 170 & 16 & 27 & 23 \\
\hline 5 & 208 & 4 & 2 & 5 & 331 & 170 & 18 & 27 & 25 \\
\hline 4 & 209 & 4 & 2 & 5 & 334 & 171 & 20 & 28 & 26 \\
\hline 3 & 209 & 4 & 2 & 5 & 336 & 171 & 20 & 28 & 27 \\
\hline 2 & 210 & 4 & 2 & 5 & 338 & 172 & 21 & 29 & 28 \\
\hline 1 & 210 & 4 & 2 & 5 & 341 & 173 & 21 & 30 & 28 \\
\hline
\end{tabular}


mocness-1-cop222. int WCR-82H MOCNESS tow copepods

$4 q-6$

net\# ifspi imspi ichet ifhal imhal ichal iaugs ifhel imhel

\begin{tabular}{rrrrrrrrrr}
\hline 18 & 0 & 0 & 0 & 0 & 0 & 0 & 0 & 0 & 0 \\
17 & 0 & 0 & 4 & 0 & 0 & 0 & 0 & 0 & 0 \\
16 & 0 & 0 & 13 & 0 & 0 & 0 & 0 & 0 & 0 \\
15 & 0 & 0 & 25 & 0 & 0 & 0 & 1 & 0 & 0 \\
14 & 0 & 0 & 50 & 15 & 0 & 1 & 4 & 0 & 0 \\
13 & 0 & 0 & 73 & 33 & 0 & 1 & 12 & 0 & 0 \\
12 & 0 & 0 & 83 & 108 & 0 & 1 & 16 & 0 & 0 \\
11 & 0 & 0 & 87 & 123 & 2 & 3 & 19 & 0 & 0 \\
8 & 1 & 1 & 90 & 128 & 2 & 19 & 21 & 1 & 0 \\
7 & 1 & 1 & 94 & 131 & 2 & 50 & 22 & 1 & 0 \\
6 & 1 & 1 & 98 & 132 & 2 & 68 & 23 & 1 & 0 \\
5 & 1 & 1 & 100 & 133 & 3 & 99 & 24 & 1 & 1 \\
4 & 2 & 1 & 102 & 134 & 3 & 125 & 26 & 2 & 2 \\
3 & 2 & 2 & 103 & 136 & 3 & 147 & 27 & 2 & 2 \\
2 & 2 & 2 & 104 & 137 & 3 & 154 & 28 & 2 & 2 \\
1 & 2 & 2 & 106 & 138 & 3 & 166 & 29 & 3 & 5 \\
\hline
\end{tabular}

net\# ifcur imcur imeth ifket imket iflon imlon ifpak impak

\begin{tabular}{llllllllll}
\hline 18 & 0 & 0 & 0 & 0 & 0 & 0 & 0 & 0 & 1 \\
17 & 0 & 0 & 0 & 0 & 0 & 0 & 0 & 1 & 1 \\
16 & 0 & 0 & 0 & 0 & 0 & 2 & 0 & 1 & 1 \\
15 & 0 & 0 & 0 & 0 & 0 & 4 & 1 & 1 & 1 \\
14 & 0 & 0 & 0 & 0 & 0 & 5 & 3 & 1 & 2 \\
13 & 0 & 0 & 0 & 0 & 0 & 6 & 4 & 1 & 2 \\
12 & 0 & 0 & 0 & 0 & 0 & 6 & 4 & 2 & 2 \\
11 & 0 & 0 & 0 & 0 & 0 & 7 & 4 & 2 & 2 \\
8 & 0 & 0 & 0 & 0 & 0 & 7 & 4 & 2 & 2 \\
7 & 0 & 0 & 0 & 0 & 0 & 7 & 4 & 2 & 2 \\
6 & 0 & 0 & 0 & 0 & 0 & 7 & 4 & 2 & 2 \\
5 & 0 & 0 & 0 & 1 & 0 & 7 & 4 & 2 & 2 \\
4 & 0 & 0 & 0 & 1 & 0 & 7 & 4 & 2 & 2 \\
3 & 0 & 0 & 0 & 1 & 0 & 7 & 4 & 2 & 2 \\
2 & 0 & 0 & 0 & 1 & 0 & 7 & 4 & 2 & 2 \\
1 & 0 & 0 & 0 & 1 & 0 & 7 & 4 & 2 & 2 \\
\hline
\end{tabular}


mocness-1-cop222. int WCR-82H MOCNESS tow copepods

$4 q-7$

net\# ifpbi impbi iccan ifpon impon icpon ifadn imada ifneg

\begin{tabular}{rrrrrrrrrr}
\hline 18 & 4 & 3 & 4 & 0 & 0 & 1 & 15 & 0 & 0 \\
17 & 7 & 10 & 13 & 0 & 0 & 2 & 22 & 1 & 0 \\
16 & 12 & 13 & 20 & 0 & 1 & 3 & 24 & 1 & 0 \\
15 & 17 & 15 & 27 & 0 & 1 & 3 & 26 & 1 & 0 \\
14 & 19 & 16 & 37 & 1 & 1 & 6 & 28 & 1 & 0 \\
13 & 21 & 18 & 37 & 1 & 2 & 8 & 33 & 1 & 0 \\
12 & 25 & 22 & 59 & 3 & 3 & 8 & 35 & 1 & 0 \\
11 & 28 & 24 & 69 & 4 & 3 & 8 & 36 & 1 & 0 \\
8 & 30 & 27 & 73 & 4 & 3 & 8 & 36 & 1 & 1 \\
7 & 31 & 27 & 73 & 4 & 3 & 8 & 36 & 1 & 1 \\
6 & 31 & 27 & 73 & 4 & 3 & 8 & 36 & 1 & 1 \\
5 & 31 & 27 & 73 & 4 & 3 & 8 & 36 & 1 & 1 \\
4 & 31 & 27 & 73 & 4 & 3 & 8 & 36 & 1 & 1 \\
3 & 31 & 27 & 73 & 4 & 3 & 8 & 36 & 1 & 1 \\
2 & 31 & 27 & 73 & 4 & 3 & 8 & 36 & 1 & 1 \\
1 & 31 & 27 & 73 & 4 & 3 & 8 & 36 & 1 & 1 \\
\hline
\end{tabular}

net\# imneg imisc ioith icycl itotl

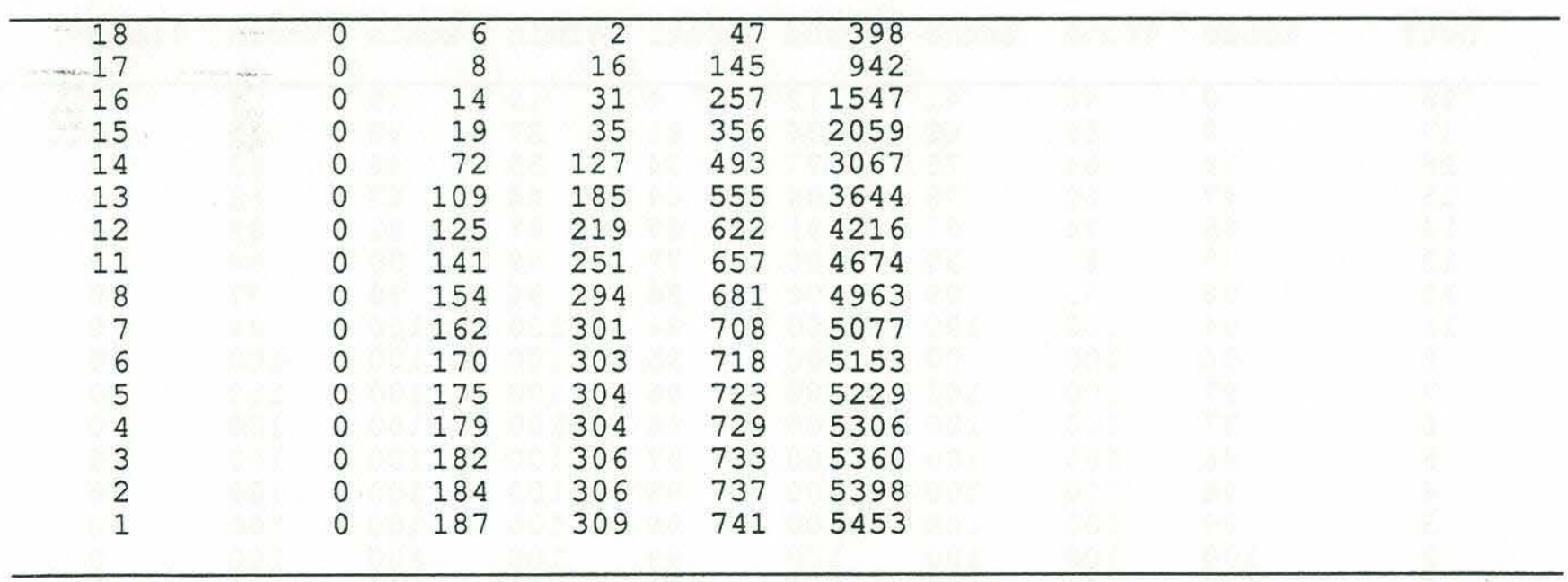


mocness-1-cop222. cum WCR-82H MOCNESS tow copepods

$4 r-1$

\begin{tabular}{rrrrrrrrrr} 
net\# & midd & depi & volf & aliq & often & smten & orcten & offnro & smnro \\
\hline 18 & 5 & 10 & 244 & 8 & 0 & 0 & 0 & 25 & 0 \\
17 & 15 & 10 & 177 & 8 & 0 & 0 & 0 & 28 & 0 \\
16 & 25 & 10 & 185 & 8 & 0 & 0 & 0 & 45 & 0 \\
15 & 35 & 10 & 227 & 8 & 1 & 0 & 2 & 55 & 0 \\
14 & 45 & 10 & 265 & 8 & 3 & 0 & 7 & 62 & 0 \\
13 & 55 & 10 & 220 & 8 & 4 & 0 & 10 & 62 & 0 \\
12 & 65 & 10 & 231 & 8 & 49 & 3 & 36 & 88 & 0 \\
11 & 75 & 10 & 339 & 8 & 93 & 16 & 80 & 94 & 0 \\
8 & 85 & 10 & 218 & 4 & 99 & 59 & 97 & 97 & 0 \\
7 & 95 & 10 & 225 & 4 & 100 & 69 & 99 & 98 & 0 \\
6 & 105 & 10 & 217 & 2 & 100 & 79 & 99 & 98 & 0 \\
5 & 115 & 10 & 167 & 1 & 100 & 91 & 99 & 99 & 0 \\
4 & 125 & 10 & 282 & 1 & 100 & 95 & 100 & 99 & 0 \\
3 & 135 & 10 & 284 & 1 & 100 & 97 & 100 & 100 & 0 \\
2 & 145 & 10 & 221 & 1 & 100 & 98 & 100 & 100 & 0 \\
1 & 155 & 10 & 181 & 1 & 100 & 100 & 100 & 100 & 100 \\
\hline
\end{tabular}

\begin{tabular}{|c|c|c|c|c|c|c|c|c|c|}
\hline net\# & scneo & \%fund & ofmund & \%cund & focal & rfmin & ommin & $\frac{\circ}{8} \mathrm{cmin}$ & offelg \\
\hline 18 & 0 & 46 & 41 & 12 & 4 & 19 & 15 & 4 & 0 \\
\hline 17 & 9 & 59 & 62 & 36 & 21 & 37 & 32 & 15 & 0 \\
\hline 16 & 19 & 64 & 75 & 77 & 34 & 53 & 46 & 43 & 0 \\
\hline 15 & 47 & 69 & 78 & 94 & 44 & 64 & 63 & 68 & 0 \\
\hline 14 & 65 & 74 & 87 & 99 & 67 & 81 & 81 & 88 & 0 \\
\hline 13 & 75 & 83 & 90 & 100 & 77 & 89 & 90 & 94 & 0 \\
\hline 12 & 86 & 95 & 99 & 100 & 86 & 96 & 98 & 97 & 0 \\
\hline 11 & 94 & 100 & 100 & 100 & 94 & 100 & 100 & 99 & 0 \\
\hline 8 & 96 & 100 & 100 & 100 & 95 & 100 & 100 & 100 & 0 \\
\hline 7 & 97 & 100 & 100 & 100 & 96 & 100 & 100 & 100 & 0 \\
\hline 6 & 97 & 100 & 100 & 100 & 96 & 100 & 100 & 100 & 0 \\
\hline 5 & 98 & 100 & 100 & 100 & 97 & 100 & 100 & 100 & 0 \\
\hline 4 & 98 & 100 & 100 & 100 & 98 & 100 & 100 & 100 & 0 \\
\hline 3 & 99 & 100 & 100 & 100 & 99 & 100 & 100 & 100 & 0 \\
\hline 2 & 100 & 100 & 100 & 100 & 99 & 100 & 100 & 100 & 0 \\
\hline 1 & 100 & 100 & 100 & 100 & 100 & 100 & 100 & 100 & 0 \\
\hline
\end{tabular}


mocness-1-cop222. cum WCR-82H MOCNESS tow copepods

$4 r \cdot 2$

\begin{tabular}{rrrrrrrrrr} 
net\# & scelg & offatt & smatt & scatt & ofmey & offacr & ofpav & ofarc & omarc \\
\hline 18 & 0 & 3 & 0 & 5 & 0 & 19 & 20 & 5 & 0 \\
17 & 0 & 26 & 9 & 13 & 0 & 79 & 46 & 10 & 27 \\
16 & 0 & 51 & 9 & 30 & 0 & 79 & 53 & 17 & 34 \\
15 & 0 & 58 & 23 & 41 & 0 & 86 & 59 & 26 & 55 \\
14 & 0 & 64 & 23 & 64 & 0 & 100 & 61 & 45 & 64 \\
13 & 0 & 75 & 60 & 74 & 35 & 100 & 61 & 55 & 75 \\
12 & 0 & 89 & 95 & 92 & 75 & 100 & 67 & 69 & 86 \\
11 & 0 & 96 & 100 & 100 & 98 & 100 & 71 & 92 & 100 \\
8 & 0 & 98 & 100 & 100 & 98 & 100 & 99 & 98 & 100 \\
7 & 0 & 99 & 100 & 100 & 98 & 100 & 99 & 98 & 100 \\
6 & 0 & 99 & 100 & 100 & 98 & 100 & 100 & 99 & 100 \\
5 & 0 & 99 & 100 & 100 & 98 & 100 & 100 & 99 & 100 \\
4 & 0 & 100 & 100 & 100 & 98 & 100 & 100 & 99 & 100 \\
3 & 100 & 100 & 100 & 100 & 99 & 100 & 100 & 100 & 100 \\
2 & 100 & 100 & 100 & 100 & 99 & 100 & 100 & 100 & 100 \\
1 & 100 & 100 & 100 & 100 & 100 & 100 & 100 & 100 & 100 \\
\hline
\end{tabular}

\begin{tabular}{|c|c|c|c|c|c|c|c|c|c|}
\hline net\# & scarc & \%furc & \&faar & \&osmaar & offege & \&cege & offgmi & $8 c g m i$ & offeme \\
\hline $\begin{array}{r}18 \\
17 \\
16 \\
15 \\
14 \\
13 \\
12 \\
11 \\
8 \\
7 \\
6 \\
5 \\
4 \\
3 \\
2 \\
1\end{array}$ & $\begin{array}{r}14 \\
47 \\
65 \\
79 \\
82 \\
86 \\
89 \\
92 \\
99 \\
99 \\
99 \\
100 \\
100 \\
100 \\
100 \\
100\end{array}$ & $\begin{array}{r}37 \\
65 \\
76 \\
79 \\
79 \\
81 \\
83 \\
83 \\
89 \\
91 \\
92 \\
92 \\
93 \\
93 \\
93 \\
100\end{array}$ & $\begin{array}{r}0 \\
0 \\
0 \\
0 \\
0 \\
0 \\
0 \\
0 \\
24 \\
48 \\
48 \\
48 \\
62 \\
100 \\
100 \\
100\end{array}$ & $\begin{array}{r}0 \\
0 \\
0 \\
0 \\
0 \\
0 \\
0 \\
7 \\
37 \\
66 \\
74 \\
76 \\
76 \\
76 \\
89 \\
100\end{array}$ & $\begin{array}{r}0 \\
0 \\
0 \\
0 \\
0 \\
0 \\
19 \\
78 \\
91 \\
96 \\
96 \\
97 \\
98 \\
99 \\
100 \\
100\end{array}$ & $\begin{array}{r}0 \\
0 \\
0 \\
0 \\
0 \\
0 \\
2 \\
33 \\
94 \\
97 \\
99 \\
100 \\
100 \\
100 \\
100 \\
100\end{array}$ & $\begin{array}{r}0 \\
0 \\
0 \\
0 \\
0 \\
0 \\
5 \\
27 \\
65 \\
70 \\
80 \\
87 \\
95 \\
97 \\
98 \\
100\end{array}$ & $\begin{array}{r}0 \\
0 \\
0 \\
0 \\
0 \\
0 \\
1 \\
5 \\
15 \\
20 \\
28 \\
40 \\
62 \\
74 \\
83 \\
100\end{array}$ & $\begin{array}{r}0 \\
8 \\
47 \\
72 \\
82 \\
82 \\
95 \\
99 \\
99 \\
99 \\
99 \\
99 \\
99 \\
99 \\
99 \\
100\end{array}$ \\
\hline
\end{tabular}


mocness-1-cop222. cum WCR-82H MOCNESS tow copepods

$4 r \cdot 3$

net\# smeme sfros scuch sfchi smchi sfupl smupl sfeuk smeuk

\begin{tabular}{rrrrrrrrrr}
\hline 18 & 0 & 0 & 0 & 0 & 0 & 13 & 0 & 20 & 33 \\
17 & 0 & 0 & 0 & 0 & 0 & 37 & 0 & 44 & 41 \\
16 & 42 & 0 & 3 & 0 & 0 & 40 & 0 & 50 & 55 \\
15 & 42 & 0 & 10 & 0 & 0 & 62 & 0 & 55 & 67 \\
14 & 72 & 0 & 63 & 0 & 0 & 71 & 0 & 63 & 74 \\
13 & 72 & 0 & 97 & 0 & 0 & 79 & 0 & 69 & 77 \\
12 & 88 & 0 & 103 & 16 & 0 & 85 & 0 & 83 & 89 \\
11 & 100 & 0 & 104 & 49 & 0 & 90 & 0 & 91 & 93 \\
8 & 100 & 0 & 105 & 75 & 0 & 93 & 0 & 95 & 99 \\
7 & 100 & 100 & 105 & 83 & 16 & 97 & 0 & 98 & 99 \\
6 & 100 & 100 & 105 & 91 & 57 & 98 & 0 & 98 & 99 \\
5 & 100 & 100 & 105 & 91 & 84 & 98 & 0 & 99 & 99 \\
4 & 100 & 100 & 106 & 95 & 94 & 99 & 0 & 99 & 100 \\
3 & 100 & 100 & 106 & 98 & 100 & 100 & 0 & 99 & 100 \\
2 & 100 & 100 & 107 & 100 & 100 & 100 & 0 & 100 & 100 \\
1 & 100 & 100 & 100 & 100 & 100 & 100 & 0 & 100 & 100 \\
\hline
\end{tabular}

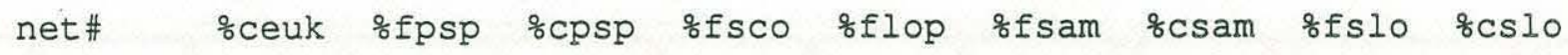

\begin{tabular}{rrrrrrrrrr}
\hline 18 & 5 & 0 & 0 & 0 & 0 & 1 & 2 & 0 & 0 \\
17 & 11 & 0 & 7 & 0 & 0 & 4 & 7 & 1 & 2 \\
16 & 21 & 0 & 14 & 0 & 0 & 11 & 25 & 12 & 8 \\
15 & 27 & 19 & 19 & 0 & 0 & 13 & 35 & 22 & 24 \\
14 & 48 & 19 & 19 & 0 & 0 & 36 & 46 & 37 & 45 \\
13 & 64 & 19 & 19 & 0 & 0 & 52 & 50 & 49 & 58 \\
12 & 71 & 56 & 52 & 24 & 36 & 74 & 66 & 64 & 75 \\
11 & 88 & 81 & 74 & 58 & 98 & 85 & 77 & 87 & 89 \\
8 & 93 & 91 & 79 & 84 & 98 & 93 & 89 & 91 & 91 \\
7 & 94 & 91 & 88 & 84 & 98 & 94 & 91 & 93 & 92 \\
6 & 96 & 91 & 95 & 90 & 98 & 94 & 93 & 94 & 93 \\
5 & 97 & 94 & 96 & 90 & 98 & 95 & 95 & 96 & 94 \\
4 & 98 & 96 & 98 & 95 & 100 & 98 & 99 & 97 & 96 \\
3 & 99 & 100 & 99 & 100 & 100 & 99 & 99 & 98 & 97 \\
2 & 100 & 100 & 100 & 100 & 100 & 99 & 100 & 99 & 98 \\
1 & 100 & 100 & 100 & 100 & 100 & 100 & 100 & 100 & 100 \\
\hline
\end{tabular}


mocness-1-cop222. cum WCR-82H MOCNESS tow copepods

$4 r 4$

\begin{tabular}{rrrrrrrrrr} 
net\# & offbra & smbra & ocbra & ofdan & omdan & ocdan & oflam & sclam & ofvit \\
\hline 18 & 0 & 0 & 0 & 23 & 9 & 7 & 0 & 0 & 0 \\
17 & 0 & 0 & 7 & 46 & 41 & 22 & 0 & 0 & 0 \\
16 & 0 & 0 & 7 & 80 & 72 & 50 & 0 & 0 & 0 \\
15 & 3 & 0 & 7 & 87 & 87 & 73 & 3 & 9 & 0 \\
14 & 20 & 0 & 17 & 91 & 100 & 81 & 44 & 39 & 0 \\
13 & 30 & 35 & 26 & 91 & 100 & 86 & 80 & 63 & 0 \\
12 & 40 & 57 & 49 & 91 & 100 & 95 & 94 & 71 & 0 \\
11 & 80 & 94 & 96 & 100 & 100 & 100 & 98 & 77 & 0 \\
8 & 88 & 94 & 99 & 100 & 100 & 100 & 98 & 78 & 0 \\
7 & 93 & 100 & 99 & 100 & 100 & 100 & 98 & 78 & 0 \\
6 & 96 & 100 & 99 & 100 & 100 & 100 & 98 & 82 & 9 \\
5 & 96 & 100 & 99 & 100 & 100 & 100 & 98 & 86 & 31 \\
4 & 97 & 100 & 99 & 100 & 100 & 100 & 99 & 90 & 49 \\
3 & 98 & 100 & 100 & 100 & 100 & 100 & 99 & 92 & 62 \\
2 & 100 & 100 & 100 & 100 & 100 & 100 & 100 & 92 & 83 \\
1 & 100 & 100 & 100 & 100 & 100 & 100 & 100 & 100 & 100 \\
\hline
\end{tabular}

net\# \%fden smsca scsca oftst omtst octst ofven omven ofbor

\begin{tabular}{rrrrrrrrrr}
\hline 18 & 13 & 2 & 4 & 44 & 78 & 24 & 0 & 0 & 0 \\
17 & 44 & 8 & 21 & 44 & 100 & 34 & 0 & 0 & 0 \\
16 & 58 & 22 & 26 & 92 & 100 & 39 & 0 & 0 & 0 \\
15 & 69 & 29 & 33 & 100 & 100 & 48 & 0 & 0 & 0 \\
14 & 73 & 39 & 35 & 100 & 100 & 91 & 0 & 0 & 0 \\
13 & 76 & 65 & 39 & 100 & 100 & 100 & 0 & 0 & 0 \\
12 & 82 & 70 & 57 & 100 & 100 & 100 & 0 & 0 & 0 \\
11 & 87 & 82 & 72 & 100 & 100 & 100 & 0 & 0 & 100 \\
8 & 90 & 82 & 91 & 100 & 100 & 100 & 0 & 0 & 100 \\
7 & 91 & 84 & 92 & 100 & 100 & 100 & 0 & 0 & 100 \\
6 & 96 & 85 & 94 & 100 & 100 & 100 & 0 & 0 & 100 \\
5 & 98 & 87 & 94 & 100 & 100 & 100 & 0 & 0 & 100 \\
4 & 99 & 91 & 95 & 100 & 100 & 100 & 0 & 0 & 100 \\
3 & 100 & 97 & 96 & 100 & 100 & 100 & 0 & 0 & 100 \\
2 & 100 & 98 & 97 & 100 & 100 & 100 & 0 & 0 & 100 \\
1 & 100 & 100 & 100 & 100 & 100 & 100 & 0 & 100 & 100 \\
\hline
\end{tabular}


mocness-1-cop222. cum WCR-82H MOCNESS tow copepods

$4 r \cdot 5$

net\# \&fgra \%mpsm ofpis ompis ocpsm \&fabd \&mabd ofxip omxip

\begin{tabular}{rrrrrrrrrr}
\hline 18 & 25 & 1 & 47 & 27 & 4 & 12 & 9 & 13 & 0 \\
17 & 53 & 3 & 69 & 53 & 16 & 15 & 18 & 18 & 0 \\
16 & 74 & 15 & 83 & 64 & 31 & 32 & 32 & 28 & 5 \\
15 & 82 & 24 & 90 & 81 & 57 & 45 & 36 & 36 & 5 \\
14 & 88 & 50 & 95 & 89 & 88 & 69 & 54 & 48 & 13 \\
13 & 94 & 59 & 97 & 95 & 93 & 83 & 66 & 56 & 27 \\
12 & 96 & 68 & 98 & 98 & 99 & 93 & 69 & 68 & 40 \\
11 & 96 & 74 & 99 & 100 & 99 & 98 & 77 & 93 & 67 \\
8 & 98 & 95 & 100 & 100 & 99 & 98 & 96 & 98 & 94 \\
7 & 98 & 96 & 100 & 100 & 99 & 98 & 97 & 98 & 94 \\
6 & 98 & 96 & 100 & 100 & 100 & 98 & 97 & 98 & 98 \\
5 & 99 & 97 & 100 & 100 & 100 & 99 & 98 & 99 & 99 \\
4 & 99 & 99 & 100 & 100 & 100 & 99 & 99 & 100 & 99 \\
3 & 99 & 100 & 100 & 100 & 100 & 99 & 99 & 100 & 99 \\
2 & 100 & 100 & 100 & 100 & 100 & 100 & 99 & 100 & 100 \\
1 & 100 & 100 & 100 & 100 & 100 & 100 & 100 & 100 & 100 \\
\hline
\end{tabular}

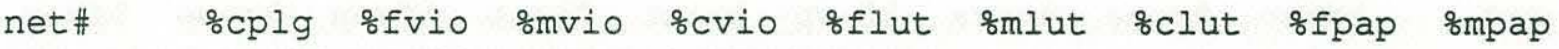

\begin{tabular}{rrrrrrrrrr}
\hline 18 & 1 & 24 & 52 & 47 & 16 & 18 & 0 & 6 & 1 \\
17 & 5 & 68 & 100 & 84 & 32 & 35 & 0 & 27 & 14 \\
16 & 16 & 100 & 100 & 93 & 46 & 46 & 4 & 40 & 34 \\
15 & 28 & 100 & 100 & 100 & 54 & 55 & 7 & 51 & 44 \\
14 & 67 & 100 & 100 & 100 & 68 & 73 & 25 & 65 & 52 \\
13 & 84 & 100 & 100 & 100 & 75 & 78 & 42 & 70 & 63 \\
12 & 91 & 100 & 100 & 100 & 83 & 89 & 45 & 76 & 69 \\
11 & 94 & 100 & 100 & 100 & 89 & 95 & 51 & 81 & 69 \\
8 & 97 & 100 & 100 & 100 & 95 & 97 & 62 & 83 & 74 \\
7 & 99 & 100 & 100 & 100 & 96 & 98 & 64 & 87 & 81 \\
6 & 99 & 100 & 100 & 100 & 97 & 98 & 76 & 90 & 83 \\
5 & 99 & 100 & 100 & 100 & 97 & 99 & 85 & 92 & 90 \\
4 & 99 & 100 & 100 & 100 & 98 & 99 & 95 & 94 & 93 \\
3 & 100 & 100 & 100 & 100 & 99 & 99 & 97 & 96 & 96 \\
2 & 100 & 100 & 100 & 100 & 99 & 99 & 98 & 98 & 98 \\
1 & 100 & 100 & 100 & 100 & 100 & 100 & 100 & 100 & 100 \\
\hline
\end{tabular}


mocness-1-cop222. cum WCR-82H MOCNESS tow copepods

$4 r-6$

net\# offspi smspi ochet ofhal omhal ochal saugs ofhel omhel

\begin{tabular}{rrrrrrrrrr}
\hline 18 & 0 & 0 & 0 & 0 & 0 & 0 & 0 & 0 & 0 \\
17 & 0 & 0 & 4 & 0 & 0 & 0 & 0 & 0 & 0 \\
16 & 17 & 22 & 12 & 0 & 0 & 0 & 1 & 0 & 0 \\
15 & 17 & 22 & 24 & 0 & 0 & 0 & 3 & 0 & 0 \\
14 & 17 & 22 & 47 & 11 & 0 & 0 & 12 & 0 & 0 \\
13 & 17 & 22 & 69 & 24 & 0 & 0 & 39 & 0 & 0 \\
12 & 17 & 22 & 78 & 78 & 11 & 1 & 55 & 0 & 0 \\
11 & 17 & 22 & 81 & 89 & 49 & 2 & 66 & 0 & 0 \\
8 & 47 & 31 & 85 & 92 & 55 & 12 & 72 & 27 & 0 \\
7 & 47 & 40 & 88 & 95 & 60 & 30 & 76 & 27 & 0 \\
6 & 51 & 49 & 92 & 95 & 75 & 41 & 79 & 30 & 0 \\
5 & 58 & 61 & 94 & 96 & 92 & 60 & 82 & 46 & 24 \\
4 & 70 & 73 & 95 & 97 & 97 & 75 & 88 & 68 & 47 \\
3 & 84 & 91 & 97 & 98 & 100 & 88 & 90 & 77 & 52 \\
2 & 93 & 100 & 98 & 99 & 100 & 93 & 96 & 90 & 52 \\
1 & 100 & 100 & 100 & 100 & 100 & 100 & 100 & 100 & 100 \\
\hline
\end{tabular}

\begin{tabular}{rrrrrrrrrr} 
net\# & sffur & smcur & smeth & sfket & smket & sflon & smlon & sfpak & smpak \\
\hline 18 & 0 & 0 & 0 & 0 & 0 & 0 & 0 & 18 & 27 \\
17 & 0 & 0 & 100 & 0 & 0 & 0 & 0 & 44 & 27 \\
16 & 0 & 0 & 100 & 0 & 0 & 24 & 0 & 44 & 44 \\
15 & 0 & 0 & 100 & 0 & 0 & 52 & 33 & 64 & 59 \\
14 & 0 & 0 & 100 & 0 & 0 & 69 & 62 & 81 & 71 \\
13 & 0 & 0 & 100 & 0 & 0 & 84 & 87 & 81 & 86 \\
12 & 0 & 0 & 100 & 0 & 0 & 84 & 96 & 100 & 100 \\
11 & 61 & 0 & 100 & 0 & 0 & 90 & 96 & 100 & 100 \\
8 & 61 & 0 & 100 & 0 & 0 & 95 & 100 & 100 & 100 \\
7 & 61 & 0 & 100 & 0 & 0 & 100 & 100 & 100 & 100 \\
6 & 85 & 0 & 100 & 38 & 0 & 100 & 100 & 100 & 100 \\
5 & 100 & 0 & 100 & 74 & 0 & 100 & 100 & 100 & 100 \\
4 & 100 & 0 & 100 & 89 & 12 & 100 & 100 & 100 & 100 \\
3 & 100 & 0 & 100 & 89 & 48 & 100 & 100 & 100 & 100 \\
2 & 100 & 0 & 100 & 89 & 63 & 100 & 100 & 100 & 100 \\
1 & 100 & 0 & 100 & 100 & 100 & 100 & 100 & 100 & 100 \\
\hline
\end{tabular}


mocness-1-cop222. cum WCR-82H MOCNESS tow copepods

$4 r-7$

net\# \%fpbi \&mpbi sccan \%fpon smpon scpon sfadn smada \%fneg

\begin{tabular}{rrrrrrrrrr}
\hline 18 & 12 & 10 & 6 & 8 & 0 & 8 & 40 & 42 & 0 \\
17 & 22 & 38 & 18 & 8 & 0 & 25 & 62 & 100 & 0 \\
16 & 39 & 47 & 27 & 8 & 28 & 35 & 66 & 100 & 0 \\
15 & 55 & 56 & 37 & 8 & 39 & 39 & 70 & 100 & 0 \\
14 & 63 & 58 & 50 & 16 & 39 & 69 & 76 & 100 & 0 \\
13 & 69 & 66 & 50 & 25 & 51 & 96 & 92 & 100 & 0 \\
12 & 81 & 81 & 81 & 70 & 85 & 100 & 95 & 100 & 0 \\
11 & 90 & 89 & 94 & 100 & 100 & 100 & 99 & 100 & 0 \\
8 & 97 & 98 & 99 & 100 & 100 & 100 & 100 & 100 & 60 \\
7 & 100 & 99 & 99 & 100 & 100 & 100 & 100 & 100 & 60 \\
6 & 100 & 100 & 99 & 100 & 100 & 100 & 100 & 100 & 70 \\
5 & 100 & 100 & 99 & 100 & 100 & 100 & 100 & 100 & 76 \\
4 & 100 & 100 & 100 & 100 & 100 & 100 & 100 & 100 & 84 \\
3 & 100 & 100 & 100 & 100 & 100 & 100 & 100 & 100 & 88 \\
2 & 100 & 100 & 100 & 100 & 100 & 100 & 100 & 100 & 88 \\
1 & 100 & 100 & 100 & 100 & 100 & 100 & 100 & 100 & 100 \\
\hline
\end{tabular}

net\# omneg omisc orith scycl stotl

\begin{tabular}{rrrrrr}
\hline 18 & 0 & 3 & 1 & 6 & 7 \\
17 & 0 & 4 & 5 & 20 & 17 \\
16 & 0 & 8 & 10 & 35 & 28 \\
15 & 0 & 10 & 11 & 48 & 38 \\
14 & 0 & 38 & 41 & 66 & 56 \\
13 & 0 & 58 & 60 & 75 & 67 \\
12 & 0 & 67 & 71 & 84 & 77 \\
11 & 0 & 76 & 81 & 89 & 86 \\
8 & 100 & 83 & 95 & 92 & 91 \\
7 & 100 & 86 & 98 & 95 & 93 \\
6 & 100 & 91 & 98 & 97 & 95 \\
5 & 100 & 94 & 98 & 98 & 96 \\
4 & 100 & 96 & 99 & 98 & 97 \\
3 & 100 & 97 & 99 & 99 & 98 \\
2 & 100 & 98 & 99 & 99 & 99 \\
1 & 100 & 100 & 100 & 100 & 100 \\
\hline
\end{tabular}


Appendix 1: Miscellaneous Calanoids:

The following lists some of the animals included under the 'misc' heading in the abundance tables. It is not complete but gives abundances and sex/stage (female $=f$, male $=m$, copepodite $=\mathrm{c}$ ) where available. The values are for the number of individuals found in the aliquot sorted ('raw data'). Some notes on relative abundance, appearance, and body length are also given.

\section{MOC 217}

Mecynocera, Calocalanus parvo, Clausocalanus arcuicornis, C.furcatus, Scolecithrix spp., Temora spp. copepodites were always included in 'misc' in this tow.

Net $0(0-123 \mathrm{~m})$ : Acrocalanus longicornis, Clausocalanus furcatus, 57 Scolecithrix spp. copepodites

Net $1(123-73 \mathrm{~m})$ : Acrocalanus longicornis, Clausocalanus furcatus, $1 \mathrm{~m}$ Euchaeta spinosa, $1 \mathrm{f}$ Euaetideus giesbrechti, 1 Megacalanus longicornis, 56 c Scolecithrix danae and S. bradyi

Net 2 (73-44 m): 22 c Scolecithrix danae and S. bradyi

Net 3 (44-0 m): 32 f Acrocalanus longicornis, 44 c Scolecithrix danae and S. bradyi, 9 c Temora spp., 1 c Pontellina plumata

Net $4(0-126 \mathrm{~m})$ : many c Scolecithrix spp.

Net 5 (126-77 m): f Clausocalanus furcatus, $1 \mathrm{~m}$ Euchaeta sp., many c Scolecithrix danae and $S$. bradyi

Net $6(77-39 \mathrm{~m}): 2 \mathrm{f}$ and $3 \mathrm{c}$ Eucalanus attenuatus, $1 \mathrm{f}$ Acrocalanus longicornis, $6 \mathrm{c}$ Scolecithrix danae

Net 7 (39-0 m): 11 f Acrocalanus longicornis, c Scolecithrix danae and S. bradyi, c Temora spp.

Net $8(0-120 \mathrm{~m}): 2$ f Aetideus armatus, 28 c Scolecithrix danae, 1 cPontellina plumata

Net 11 (120-74 m): 2 f Acrocalanus longicornis, many c Scolecithrix spp.

Net 12 (74-27 m): $1 \mathrm{f}$ and c Eucalanus attenuatus, f Clausocalanus furcatus, c Scolecithrix spp.

Net 17 (23-0 m): f Clausocalanus furcatus

Net 19 (134-0 m): 4 f Aetideus armatus, 1 f Scolecithricella vittata 


\section{MOC 218}

Copepodites of Euchaeta spp., Scolecithrix danae and S. bradyi, Heterorhabdus spp., Metridia spp., Candacia spp. were always included in 'misc'.

Net 18 (0-25 m): Eucalanus attenuatus, Eucalanus $n r$. monachus, Clausocalanus arcuicornis, 209 c Euchaeta spp., 4 c Phaenna spinifera, Scaphocalanus nr. amplius, S. nr. longifurcus, Scolecithricella dentata, 3 f Temora stylifera, 3 f Temora turbinata, 29 c Centropages violaceus, 17 c Centropages typicus, $1 \mathrm{~m}$ Candacia ethiopica, $1 \mathrm{f}$ Pontella sp., 53 c Candacia spp., 22 c Pontellina plumata

Net $17(25-50 \mathrm{~m}): 1 \mathrm{f}$ Temora stylifera, 1 Candacia paenelongimana

Net 16 (50-75 m): f Eucalanus sp., 1 Euchaeta paraconcinna, f Scaphocalanus $\mathrm{nr}$. longifurcus, f Scolecithricella dentata, c Centropages spp., 1 f Pontellina plumata

Net $15(75-100 \mathrm{~m})$ : many $\mathrm{f}$ and some $\mathrm{m}$ Aetideus armatus, $2 \mathrm{f}$ Euchirella rostrata, f Lophothrix latipes, f Scaphocalanus nr. longifurcus, c Scolecithrix danae and S. bradyi

Net 14 (100-125 m): 1 Euchirella rostrata, 1 f Eucheata bitumida

Net 13 (125-150 m): 4 f Scottocalanus sp. nr. australis (compared with S. securifrons, these females are smaller, the genital segment does not overhang the next segment and corners of 5th thoracic segment are less pointed), $1 \mathrm{~m}$ Pontellina plumata

Net 12 (150-175 m): f Aetideus armatus, 1 f Euchirella rostrata, 1 f Scottocalanus sp. nr. australis, f Scaphocalanus spp., f Scolecithricella vittata, f $S$. spp.

Net 11 (175-200 m): f sp. nr. Mimocalanus (not seen in upper nets), f Gaetanus minor, $f$ Amallothrix $n$ r. laminata, f Scaphocalanus nr. amplius, f $S$. $\mathrm{nr}$. curtus, f $S$. $\mathrm{nr}$. longifurcus, f Scolecithricella vittata, f $S$. dentata

Net 8 (200-300 m): f sp. nr. Mimocalanus, f Euaetideus giesbrechti, f Gaetanus miles, f Scaphocalanus nr. longifurcus, f $S$. nr. curtus, f $S$. nr. amplius, f Scolecithricella dentata, f $S$. vittata, (f Neocalanus robustior found in sample but not present in this aliquot)

Net 7 (300-400 m): many m Clausocalanus spp., 2 f Gaetanus miles, 1 f Euchaeta intermedia, f Scaphocalanus spp., f Augaptilidae spp.

Net $6(400-500 \mathrm{~m}): 2$ c Eucalanus attenuatus, $\mathrm{f}$ sp. $\mathrm{nr}$. Mimocalanus, $3 \mathrm{f}$ Gaetanus miles, 1 $\mathrm{m}$ Euchirella nr. curticauda, Spinocalanus nr. spinosus, Scaphocalanus spp., Scolecithricella spp., c Heterorhabdus spp., Augaptilidae spp.

Net $5(500-600 \mathrm{~m}): 4$ c Eucalanus attenuatus, mostly f Spinocalanus $\mathrm{nr}$. spinosus, $1 \mathrm{f}$ Eucalanus nr. monachus, f Scaphocalanus nr. longifurcus, Augaptilidae spp. 
Net $4(600-700 \mathrm{~m}): 23$ c Eucalanus attenuatus, 2 f Gaetanus miles, $2 \mathrm{f}$ G. pileatus, $1 \mathrm{f}$ Euchaeta pseudotonsa, 2 E. incisa, $1 \mathrm{~m}$ Scottocalanus persecans, 1 cIV Metridia princeps, 2 m Heterorhabdus abysallis, $1 \mathrm{f}$ Pachyptilus eurygnathus, many f Spinocalanus $\mathrm{nr}$. spinosus

Net $3(700-800 \mathrm{~m}): 35$ c Eucalanus attenuatus, $3 \mathrm{f}$ and c Eucalanus $\mathrm{nr}$. monachus, $3 \mathrm{~m}$ Chirundina Ostreetsi, 1 Euchaeta hanseni (red mouthparts, $8.1 \mathrm{~mm}$ ), $53 \mathrm{f}$ and $\mathrm{m}$ and c Spinocalanus $\mathrm{nr}$. spinosus, $6 \mathrm{f}$ and $4 \mathrm{~m}$ Heterorhabdus abysallis

Net $2(800-900 \mathrm{~m}): 1 \mathrm{f}$ and $36 \mathrm{c}$ Eucalanus attenuatus, $3 \mathrm{f} \mathrm{E}$. monachus, $1 \mathrm{f}(4.6 \mathrm{~mm})$ and $1 \mathrm{~m}$ Gaetanus kruppi, $1 \mathrm{~m}$ Undeuchaeta major, 22 Spinocalanus $\mathrm{nr}$. spinosus, $1 \mathrm{f}$ Temora turbinata, $1 \mathrm{c}$ Centropages typicus, $3 \mathrm{f}$ and $3 \mathrm{~m}$ Heterorhabdus abysallis, $1 \mathrm{f}$ Phyllopus impar

Net $1(900-1000 \mathrm{~m}): 6 \mathrm{f}$ and many c Eucalanus $\mathrm{nr}$. monachus, $20 \mathrm{f}$ Eucalanus attenuatus, 2 f Gaetanus kruppi (4.87, $5.03 \mathrm{~mm}), 2$ f Euchaeta bisinuata $(5.03,5.36 \mathrm{~mm}), 4 \mathrm{~m} \mathrm{E}$. norvegica $(5.03,5.85(2), 6.01 \mathrm{~mm}), 1 \mathrm{f}$ Pseudochirella sp.(red, $6.09 \mathrm{~mm}$, leg 4-basipod 1 has 6 strong spines), f Scaphocalanus spp., 15 f Spinocalanus nr. spinosus, 1 f Metridia princeps, $4 \mathrm{f}$ Heterorhabdus abysallis, Augaptilidae spp., $1 \mathrm{~m}$ Candacia armata (? - missing antenna 1), many exosleletons - not counted.

\section{MOC 219}

copepodites of Eucalanus attenuatus and Eucalanus elongatus were always included in miscellaneous.

Net $18(0-25 \mathrm{~m})$ : c Scolecithrix danae, $2 \mathrm{~m}$ Temora stylifera, c Temora spp., c Centropages violaceus, c Candacia spp., c Pontellina plumata

Net $17(25-50 \mathrm{~m})$ : c Clausocalanus arcuicornis, c Acrocalanus longicornis, 1 Euchaeta paraconcinna $(2.43 \mathrm{~mm}), 2$ f Amallothrix $\mathrm{nr}$. laminata, c Scolecithrix danae, c Temora stylifera, c Lucicutia spp., many c Candacia spp.

Net 16 (50-75 m): c Eucalanus attenuatus, c Acrocalanus longicornis, c Euaetideus giesbrechti, $5 \mathrm{f}$ Scolecithricella ctenopus, $22 \mathrm{f}$ Amallothrix $\mathrm{nr}$. laminata, $1 \mathrm{~m}$ Temora stylifera, c Temora spp., c Candacia spp., $3 \mathrm{~m}$ Pontellina plumata

Net 15 (75-100 m): 1 c Eucalanus elongatus, c Euaetideus giesbrechti, $1 \mathrm{f}$ Euchaeta pubera, c Lophothrix latipes. $5 \mathrm{f}$ and c Amallothrix nr. laminata, c Lucicutia spp., many Sapphirina

Net 14100-125 m): c Euaetideus giesbrechti, 2 f Amallothrix nr. laminata (0.98 mm - smaller than other Amallothrix $\mathrm{nr}$. laminata, $2.3 \mathrm{~mm}$ but similar morphology), $1 \mathrm{f}$ Scolecithricella ctenopus, $1 \mathrm{f}$ Centropages typicus, $1 \mathrm{f}$ Paracandacia simplex

Net 13 (125-150 m): c Euaetideus giesbrechti

Net $12(150-175 \mathrm{~m}): 1 \mathrm{f} \mathrm{nr}$. Ctenocalanus sp.(0.98 mm), $1 \mathrm{f} \mathrm{nr.} \mathrm{Farrania} \mathrm{oblonga}(1.78 \mathrm{~mm})$, $1 \mathrm{f} \mathrm{nr}$. Aetideidae (1.27 mm), c Euaetideus giesbrechti, c Gaetanus minor, c Phaenna spinifera 
Net 11 (175-200 m): $1 \mathrm{~m}$ Eucalanus attenuatus, c Gaetanus minor, c Phaenna spinifera, $1 \mathrm{~m}$ Scaphocalanus magnus ? (3.73 mm; poor cond.), $1 \mathrm{~m} \quad S$. sp., $1 \mathrm{~m} \mathrm{nr}$. Xanthocalanus (2.16 $\mathrm{mm}), 1 \mathrm{f}$ Scolecithricella ctenopus (1.3 mm), $1 \mathrm{f}$ Arietellus setosus

Net 8 (200-300 m): c Gaetanus minor, 1 m Phaenna spinifera, c Lucicutia spp.

Net 7(300-400 m): $4 \mathrm{f}$ and $1 \mathrm{c}$ Gaetanus miles, $1 \mathrm{c}$ Phaenna spinifera, $2 \mathrm{f}$ Lophothrix latipes $(3.07 \mathrm{~mm}), 3 \mathrm{f}$ Scolecithricella $\mathrm{nr}$. abyssalis $(1.96 \mathrm{~mm})$, c Scolecithrix bradyi, c Augaptilidae spp., $1 \mathrm{f}$ nr. Heterorhabdus

Net 6(400-500 m): many c Spinocalanus nr. spinosus, 4 f Gaetanus miles, c Gaetanus minor, $1 \mathrm{f}$ Racovitzanus sp. $(1.78 \mathrm{~mm})$, many c Scaphocalanus sp., $2 \mathrm{~m}$ and c Haloptilus ornatus, $1 \mathrm{~m}$ Paraugaptilus sp. $(2.83 \mathrm{~mm})$

Net 5 (500-600 m): 2 c Eucalanus elongatus, c Spinocalanus nr. spinosus, c Gaetanus minor, c Euchirella spp., c Scottocalanus securifrons, c Scolecithricella spp., 1 f sp. $\mathrm{nr}$. Metridia/Pleuromamma (poor cond.), c Haloptilus ornatus, c Augaptilidae spp., 1 f Candacia spp. paenelongimana, 8 c C. spp.

Net $4(600-700 \mathrm{~m}):$ c Eucalanus $n r$. monachus, 6 c Eucalanus elongatus, $1 \mathrm{f}$ and c Spinocalanus sp., $1 \mathrm{f}$ sp. nr. Farrania oblonga $(1.33 \mathrm{~mm})$, c Gaetanus minor, $2 \mathrm{f}$ Scottocalanus securifrons, 1 c Candacia spp.

Net 3 (700-800 m): 17 c Eucalanus elongatus, c Spinocalanus nr. spinosus, c sp. nr. Mimocalanus, 5 f Spinocalanus spp., $1 \mathrm{f}$ Gaetanus miles, $7 \mathrm{~m}$ Gaidius tenuispinus (these may be Gaetanus minor which is undescribed. 5th legs of both genera are very similar; no female G. teniuspinus found), $1 \mathrm{~m}$ Scottocalanus helenae, $2 \mathrm{f} S$. securifrons, $2 \mathrm{f}(1.7,1.86 \mathrm{~mm})$ and $2 \mathrm{~m}(2.15 \mathrm{~mm})$ Scolecithridae spp., $1 \mathrm{f}$ Amallothrix emarginata, $1 \mathrm{~m}$ Augaptilidae spp.

Net 2 (800-900 m): 38 с Eucalanus attenuatus/monachus, 11 c Eucalanus elongatus, 14 c Spinocalanus nr. spinosus, 3 c Monacilla typica, 28 c Euchirella/Chirundina/Undeuchaeta, 3 $\mathrm{f}$ and $10 \mathrm{c}$ Aetideopsis multiserrata, $3 \mathrm{~m}$ Gaidius tenuispinus, $1 \mathrm{~m}$ Gaetanus pileatus, $5 \mathrm{c}$ Gaetanus spp., 1 c Lophothrix frontalis, 2 f Amallothrix emarginata, 4 c Lucicutia spp., $2 \mathrm{f}$ and $1 \mathrm{~m}$ and 3 c Disseta palumboi, 5 c Augaptilidae spp., 13 f/m/c spp. (very poor)

Net 1 (900-1000 m): 44 c Eucalanus attenuatus/monachus, 2 c Eucalanus elongatus, 12 c Spinocalanus nr. spinosus, $7 \mathrm{c}$ sp. nr. Mimocalanus, $11 \mathrm{c}$ Monacilla typica, $1 \mathrm{f}$ sp. $\mathrm{nr}$. Farrania oblonga, 1 c Gaetanus sp., 3 c Euchirella spp., 6 c Undeuchaeta plumosa, 2 c Aetideopsis multiserrata, 12 c Euchaeta spp., $1 \mathrm{f}(7.8 \mathrm{~mm})$ and 2 c Euchaeta barbata, $1 \mathrm{f}$ Euchaeta tonsa, $1 \mathrm{f}$ and $6 \mathrm{c}$ Lophothrix frontalis, $1 \mathrm{f} \mathrm{sp.} \mathrm{nr.} \mathrm{Lophothrix,} \mathrm{c} \mathrm{Scaphocalanus} \mathrm{nr}$. longifurcus, $3 \mathrm{f} S$. spp., 2 f Amallothrix $\mathrm{nr}$. valida, $1 \mathrm{~m}$ Temorites sp. $(1.75 \mathrm{~mm}), 1 \mathrm{c}$ Centropages violaceus, $1 \mathrm{~m}$ and $5 \mathrm{c}$ Disseta palumboi, $1 \mathrm{f} \mathrm{sp}$. $\mathrm{nr}$. Mesorhabdus brevicaudatus, 5 c Augaptilidae spp., $1 \mathrm{f}$ Centraugaptilus horidus, $24 \mathrm{f} / \mathrm{m} / \mathrm{c}$ spp. (very poor) 


\section{MOC 220}

Net $1(0-100 \mathrm{~m}): 1$ Euchaeta amoena

Net $4(0-100 \mathrm{~m}): 1$ c Eucalanus elongatus, 1 c Acrocalanus longicornis

Net $5(0-100 \mathrm{~m}): 1 \mathrm{c}$ probably Scolecithrix danae (molting)

Net $13(0-100 \mathrm{~m}): 1 \mathrm{f}$ nr. Farrania oblonga

Net $14(0-100 \mathrm{~m}): 1 \mathrm{f}$ Arietellus plumifer

Net $17(0-100 \mathrm{~m}): 1 \mathrm{~m}$ Mecynocera clausi

Net $18(0-100 \mathrm{~m}): 2$ c Scaphocalanus $\mathrm{nr}$. longifurcus

Net $19(0-100 \mathrm{~m}): 1$ Calanus finmarchicus CV, 2 C. finmarchicus CIV, 1 c Calocalanus parvoninus, $1 \mathrm{f}$ Undeuchaeta major, $1 \mathrm{f}$ Euchirella bitumida, 6 c Scaphocalanus $\mathrm{nr}$. longifurcus

\section{MOC 222}

Net $18(0-10 \mathrm{~m}): 1$ c Eucalanus $n r$. monachus, $1 \mathrm{~m}(0.74 \mathrm{~mm})$ and 1 c Clausocalanus furcatus, $13 \mathrm{f}$ and $1 \mathrm{~m}$ Temora turbinata

Net 17 (10-20 m): 1 c Acrocalanus longicornis (?), 1 c Temora spp.

Net $16(20-30 \mathrm{~m}): 1 \mathrm{f}$ Eucalanus $n r$. monachus, 9 c Undeuchaeta plumosa, $1 \mathrm{~m}$ Arietellus plumifer

Net 15 (30-40 m): 11 c Chirundina/Undeuchaeta

Net $14(40-50 \mathrm{~m}): 1$ c Mecynocera clausi, $171 \mathrm{c}$ Chirundina/Undeuchaeta, $3 \mathrm{f}$ and $1 \mathrm{~m}$ and 5 c Augaptilidae spp.

Net 13 (50-60 m): 1 c Mecynocera clausi, 1 f sp.nr. Ctenocalanus, 90 c Chirundina/Undeuchaeta, 1 c Lophothrix sp.

Net $12(60-70 \mathrm{~m}): 1 \mathrm{f} \mathrm{nr}$. Ctenocalanus, 34 c Chirundina/Undeuchaeta, 1 c Scottocalanus securifrons, 5 c Lophothrix spp., $1 \mathrm{~m}$ Paraugaptilus buchani, $1 \mathrm{~m}$ Arietellus plumifer

Net $11(70-80 \mathrm{~m}): 1 \mathrm{f}$ and $1 \mathrm{c}$ sp. nr. Mimocalanus, $36 \mathrm{c}$ Chirundina/Undeuchaeta, $1 \mathrm{f}$ Euchirella curticauda, $1 \mathrm{f} \mathrm{sp}$. $\mathrm{nr}$. Xanthocalanus agilis, $1 \mathrm{f} \mathrm{nr}$. Xanthocalanus/Amallothrix, 16 c Lophothrix latipes, 1 c Metridia sp.?, 1 f Arietellus plumifer, $1 \mathrm{~m}$ Candacia bipinnata

Net $8(80-90 \mathrm{~m})$ : $1 \mathrm{f} \mathrm{sp.} \mathrm{nr.} \mathrm{Mimocalanus,} 1 \mathrm{c}$ sp. nr. Farrania oblonga, $1 \mathrm{f} \mathrm{sp.} \mathrm{A,} 57 \mathrm{c}$ Chirundina /Undeuchaeta, $1 \mathrm{~m}$ Phaenna spinifera, $1 \mathrm{f}$ Scaphocalanus $\mathrm{nr}$. curtus (1.02 mm), $2 \mathrm{~m}$ and $1 \mathrm{c}$ Scottocalanus securifrons, 2 f Scolecithricella minor

Net 7 (90-100 m): 2 c sp. A, 34 c Chirundina/Undeuchaeta, 1 c Aetideus armatus, 1 f Temora turbinata

Net $6(100-110 \mathrm{~m}): 1 \mathrm{f}$ sp. nr. Mimocalanus, $2 \mathrm{f}$ and $1 \mathrm{c}$ Farrania oblonga, $5 \mathrm{f}$ and $6 \mathrm{c}$ sp. A, 76 c Chirundina/Undeuchaeta, 1 c Lophothrix latipes, $1 \mathrm{~m}$ Scaphocalanus $\mathrm{nr}$. longifurcus 
Net $5(110-120 \mathrm{~m}): 1 \mathrm{f}$ and 2 c Farrania oblonga, $4 \mathrm{f}$ and $3 \mathrm{c}$ sp. A, $71 \mathrm{c}$ Chirundina/Undeuchaeta, $1 \mathrm{f}$ Scolecithricella minor

Net $4(120-130 \mathrm{~m}): 2 \mathrm{~m} \mathrm{sp} . \mathrm{nr}$. Ctenocalanus, $4 \mathrm{f} \mathrm{sp}$. nr. Mimocalanus, $5 \mathrm{f} \mathrm{sp.} \mathrm{nr.} \mathrm{Farrania}$ oblonga, $10 \mathrm{f}$ sp. A, 1 c Aetideus armatus, 77 c Chirundina/Undeuchaeta, $2 \mathrm{~m}$ Scaphocalanus nr. longifurcus

Net 3 (130-140 m): $10 \mathrm{~m}$ sp.nr. Ctenocalanus $(1.04,1.08 \mathrm{~mm}), 5 \mathrm{f}$ sp. nr. Mimocalanus, 1 f sp. nr. Farrania oblonga $(1.52 \mathrm{~mm}), 2$ c Aetideus armatus $(1.8 \mathrm{~mm}), 1 \mathrm{f}$ and $4 \mathrm{c} \mathrm{sp} . \mathrm{A}, 50$ c Chirundina/Undeuchaeta, 1 f Euchaeta intermedia, 2 f Scolecithricella minor

Net $2(140-150 \mathrm{~m}): 1 \mathrm{~m}$ Calocalanus parvoninus $(1.26 \mathrm{~mm}), 1 \mathrm{c}$ sp. $\mathrm{nr}$. Microcalanus, $7 \mathrm{f}$ and 1 c sp. nr. Mimocalanus, 2 f sp. A, 7 c Chirundina/Undeuchaeta, 1 f Undeuchaeta major, $1 \mathrm{f}$ Euchaeta intermedia, 15 c Phyllopus helgae, $1 \mathrm{f} \mathrm{sp.?} \mathrm{(10.3} \mathrm{mm)}$

Net $1(150-160 \mathrm{~m})$ : 1 c Eucalanus nr. monachus, 1 c Eucalanus sp., $1 \mathrm{f}$ sp. nr. Microcalanus $(0.7 \mathrm{~mm}), 1 \mathrm{~m}$ sp.nr. Ctenocalanus, $5 \mathrm{f} \mathrm{sp}$. nr. Mimocalanus, $1 \mathrm{c}$ Aetideus armatus, $1 \mathrm{c}$ Euchirella messinensis, $1 \mathrm{c}$ sp. A, 33 c Euchirella/Chirundina/Undeuchaeta, $2 \mathrm{f}$ and $1 \mathrm{~m}$ Pseudochirella sp., $3 \mathrm{~m}$ Scaphocalanus $\mathrm{nr}$. longifurcus, $3 \mathrm{c} \mathrm{nr}$. Metridia brevicauda 
DOCUMENT LIBRARY

July 5, 1989

Distribution List for Technical Report Exchange

Attn: Stella Sanchez-Wade

Documents Section

Scripps Institution of Oceanography

Library, Mail Code C-075C

La Jolla, CA 92093

Hancock Library of Biology \& Oceanography

Alan Hancock Laboratory

University of Southern California

University Park

Los Angeles, CA 90089-0371

Gifts \& Exchanges

Library

Bedford Institute of Oceanography

P.O. Box 1006

Dartmouth, NS, B2Y 4A2, CANADA

Office of the International

Ice Patrol

c/o Coast Guard R \& D Center

Avery Point

Groton, CT 06340

Library

Physical Oceanographic Laboratory

Nova University

8000 N. Ocean Drive

Dania, FL 33304

NOAA/NESDIS Miami Library Center

4301 Rickenbacker Causeway

Miami, FL 33149

Library

Skidaway Institute of Oceanography

P.O. Box 13687

Savannah, GA 31416

Institute of Geophysics

University of Hawaii

Library Room 252

2525 Correa Road

Honolulu, HI 96822

Library

Chesapeake Bay Institute

4800 Atwell Road

Shady Side, MD 20876

MIT Libraries

Serial Journal Room 14E-210

Cambridge, MA 02139
Director, Ralph M. Parsons Laboratory

Room 48-311

MIT

Cambridge, MA 02139

Marine Resources Information Center

Building E38-320

MIT

Cambridge, MA 02139

Library

Lamont-Doherty Geological Observatory

Colombia University

Palisades, NY 10964

Library

Serials Department

Oregon State University

Corvallis, OR 97331

Pell Marine Science Library

University of Rhode Island

Narragansett Bay Campus

Narragansett, RI 02882

Working Collection

Texas A\&M University

Dept. of Oceanography

College Station, TX 77843

Library

Virginia Institute of Marine Science

Gloucester Point, VA 23062

Fisheries-Oceanography Library

151 Oceanography Teaching Bldg.

University of Washington

Seattle, WA 98195

Library

R.S.M.A.S.

University of Miami

4600 Rickenbacker Causeway

Miami, FL 33149

Maury Oceanographic Library

Naval Oceanographic Office

Stennis Space Center

NSTL, MS 39522-5001

Marine Sciences Collection

Mayaguez Campus Library

University of Puerto Rico

Mayagues, Puerto Rico 00708 



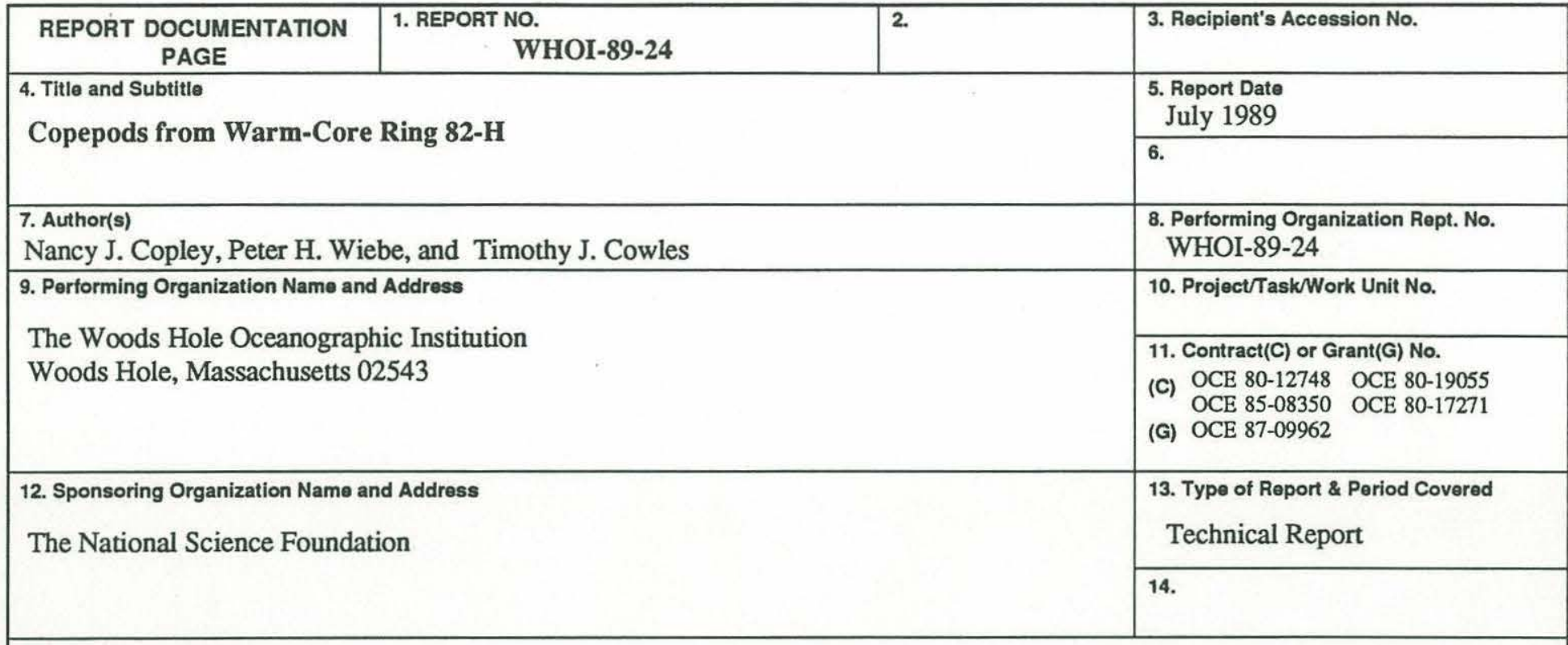

15. Supplementary Notes

This report should be cited as: Woods Hole Oceanog. Inst. Tech. Rept., WHOI-89-24.

16. Abstract (Limit: 200 words)

Net tows were collected with a Multiple Opening/Closing Net Environmental Sampling System (MOCNESS) carrying twenty $1-\mathrm{m}^{2}$ nets in October 1982 in and near warm-core ring 82-H in the North Atlantic (R/V Knorr cruise 98). This report includes the species list and abundance tables of the copepods found in five of the tows. There are four types of abundance tables: raw data, standardized to $\# / 1000 \mathrm{~m}^{3}$, integrated $\# / \mathrm{m}^{2}$ to $1000 \mathrm{~m}$ depth, and cumulative percents over the depth of the tows.

\section{Document Analysis a. Descriptors}

1. copepods/zooplankton

2. warm-core ring

3. MOCNESS

b. Identifiers/Open-Ended Terms

c. COSATI Field/Group

18. Availability Statement

Approved for publication; distribution unlimited.

19. Security Class (This Report) UNCLASSIFIED

20. Security Class (This Page)
21. No. of Pages 140

22. Price 
